\title{
Analyse des Sportunterrichts in der Arabischen Republik Ägypten
}

\author{
Dissertation \\ zur Erlangung des sozialwissenschaftlichen Doktorgrades \\ der Sozialwissenschaftlichen Fakultät \\ der Georg-August-Universität Göttingen
}

\author{
vorgelegt von \\ ASHRAF OSMAN ABD EL MOTLEB \\ aus \\ EI MANSOURA / Ägypten
}

Göttingen

2004 
Erstgutachter: Professor Dr. HERBERT HOPF Zweitgutachter: Professor Dr. JÜRGEN SCHRÖDER Tag der mündlichen Prüfung 13.10. 2004 


\section{INHALTSVERZEICHNIS}

1. Einleitung 1

$\begin{array}{lll}\text { 2. } & \text { Aspekte der ägyptischen Kultur und des Sports } & 7\end{array}$

2.1 Geographische, politische und volkswirtschaftliche Bedingungen 8

$2.2 \quad$ Allgemeine kulturelle Bedingungen 11

2.2.1 Islam und Sport 11

2.2.1.1 Grundlagen des Islams 13

2.2.1.2 Islamische Aussagen zu Bewegung, Spiel und Sport 16

2.2.1.3 Historische Entwicklung der islamischen Bewegungskultur 18

$\begin{array}{lll}2.2 .2 & \text { Familie und Sport } & 22\end{array}$

$2.3 \quad$ Außerschulisches Sportsystem 23

2.3.1 Historische Entwicklung 23

2.3.2 Gegenwärtige Struktur 26

2.3.2.1 Nationales Olympisches Komitee 27

2.3.2.2 Sportverbände 28

2.3.2.3 Sportvereine 29

$\begin{array}{lll}\text { 2.3.2.4 } & \text { Talentförderung } & 31\end{array}$

2.4 Schulsystem unter besonderer Berücksichtigung des Sportunterrichts 33

$\begin{array}{lll}2.4 .1 & \text { Historischer Abriss } & 33\end{array}$

2.4.2 Gegenwärtige Struktur 37

$\begin{array}{lll}2.5 & \text { Fazit } & 46\end{array}$

3. Der ägyptische Lehrplan für Sportunterricht, Schulsport und Sportlehrerausbildung $\quad 48$

3.1 $\quad$ Eigenschaften und Aufgaben des Sportlehrers 49

$3.2 \quad$ Erziehungsziele 51

3.2.1 Freizeit 53

3.2.2 Gesundheit 53

3.2.3 Sozialverhalten 55

$\begin{array}{lll}\text { 3.2.4 Psychische Eigenschaften } & 57\end{array}$ 
4. Fazit 
6.

$\begin{array}{ll}\text { Ausblick } & 161\end{array}$

7.

Zusammenfassung

165

Literaturverzeichnis

170

Abbildungsverzeichnis

180

Tabellenverzeichnis

Anhang

Fragebogen

187

Lebenslauf

209

Danksagung

210

Versicherung

211 


\section{Einleitung}

Die Schule gilt in nahezu allen Kulturen als wichtigste Erziehungsinstanz; in ihr sollen Fähigkeiten und Fertigkeiten vermittelt werden, auf deren Grundlage die Heranwachsenden aktuelle und zukünftige gesellschaftliche Anforderungen bewältigen können. Mit einer solchen Struktur tritt der Erziehungsgedanke hervor. Weil die Verständnisse von Erziehung stark differieren, sei der Erziehungsbegriff hier zunächst mit WENZEL (1996) umrissen:

1. Erziehung setzt ein abwärts gerichtetes Interaktionsverhältnis von ,Lehrendem' zu ,Lernendem' voraus.

2. Erziehung ist immer intentional und zweckbestimmt. Erzieherisches Handeln ist zielgerichtetes Handeln.

3. Erziehung möchte wirksam sein. Es stellt sich allerdings die Frage, ob erst dann von Erziehung gesprochen werden kann, wenn die Wirksamkeit auch nachgewiesen ist. Dies ist aber kurzfristig häufig sehr schwer, weil Wirkungen oft erst langfristig eintreten.

4. Schließlich wirken viele funktionale Erziehungsphänomene, die nur schwer zu kontrollieren sind. Dazu gehören beispielsweise Medien, soziale Gruppen und Sportvereine. Sie wirken als „heimliche“, aber durchaus wichtige Erzieher.

Will man nun die Qualität von Erziehungsinstanzen beurteilen, können drei Reflexionsebenen herangezogen werden: Gesellschaftliche Bedingungen mit ihren Anforderungen (Erziehungsziele), die Vermittlung solcher Ziele (Lehren bzw. Unterrichten) und die erzielten Lernergebnisse. Daran wird erkennbar, dass der Prozess der Vermittlung, der innerhalb der Pädagogik i.A. mit dem Begriff der Didaktik gefasst wird, das zentrale Verbindungsstück zwischen Zielen und Ergebnissen darstellt. Eine durch den Lehrer angewandte zweckmäßige Didaktik schafft es dementsprechend, die Erziehungsziele planmäßig und strukturiert bis zu den Schülern zu transportieren; dies bedeutet auch, dass eine solche Didaktik aus den zuvor reflektierten Erziehungszielen gespeist werden muss. Eine solche Sichtweise lehnt sich an die „Theorie der kategorialen Bildung“ (KLAFKI, 1963) an, die Unterrichtsplanung zum Gegenstand der Didaktik machte und über Bildungskategorien eine enge Verbindung zwischen Erziehungszielen und -inhalten zu schaffen suchte. 
Damit scheint eine erste Voraussetzung für das Erreichen von Erziehungszielen gegeben. Ist dies nicht der Fall, so können wegen häufig verfehlter Ergebnisse auch die grundsätzlich legitimierenden Aspekte des Ausbildungssystems Schule fraglich werden.

Auch der Sportunterricht als Teil eines solchen Systems kann davon betroffen sein. In der Sportpädagogik wird der Sport als Gegenstand von Bildung und Erziehung innerhalb der Institution Schule betrachtet und Fragen spezifischer Entwicklungsförderung beantwortet (vgl. BRÄUTIGAM, 2003; GRÖßING, 1997). Ein erziehender Sportunterricht versteht sich dabei als Bildungsentwurf, der nicht nur fachimmanente Ziele (wie die Vermittlung sportmotorischer Fähigkeiten und Fertigkeiten) verfolgt, sondern auch fachübergreifende Beiträge zu erbringen vermag. So gelten als wichtige legitimierende Ziele eines Sportunterrichts auch die Gesundheitserziehung und die Förderung eines aktiven Sporttreibens über die Schulzeit hinaus. Gesundheitserziehung ist dabei mehrdimensional zu verstehen, impliziert zumindest eine Aufmerksamkeitslenkung auf gesundheitlich bedeutsame Situationen, akzentuiert entsprechende Inhalte im Unterricht und fördert fachübergreifende Initiativen. Grundlagen für ein aktives Sporttreiben über die Schulzeit hinaus sind entsprechende Fähigkeiten und Fertigkeiten sowie die Förderung einer Sichtweise von Sport, die inn als sinnhafte und freudvolle Tätigkeit umschreibt. Fachimmanente Ziele beziehen sich auf körperliche Aspekte wie Haltung oder Bewegungskoordination, auf psychische Aspekte wie die Steigerung des Selbstwertgefühls und auf soziale Aspekte der teilnehmenden Einbindung. Letztere ergibt sich durch die Gelegenheit zu intensiver Interaktion. In keinem anderen Unterrichtsfach kann ein fairer Umgang miteinander und das Beachten von Regeln so eingeübt werden, denn hier liegt die Betonung auf dem Miteinander, auf einem problematisiertem Gegeneinander und führt so möglichst zur Einsicht verantwortlichen Handelns. Ein solcher Sportunterricht unterstützt im Gesamtsystem Schule eine vielfach gewünschte Entwicklungsförderung.

Die Sportdidaktik als Teilgebiet der Sportpädagogik beschäftigt sich mit Fragen des Lehrens und Lernens im Sportunterricht (vgl. BRÄUTIGAM, 2003). Als Fach geht es ihr um die Erfassung und Deutung des dortigen Geschehens, um dieses besser zu verstehen und gestalten zu können. Ihre Forschungsaktivitäten beinhalten zwei grundlegende Zielrichtungen: 1. Die Formulierung normativer Aussagen zur Gestaltung des Unterrichts (beispielsweise zu Erziehungszielen, Lehrplänen, 
Methodik und Evaluation) und 2. eine empirisch-analytische Arbeit, die in erster Linie von den Lehrern ausgeht.

Sowohl die Formulierung normativer Aussagen als auch die empirisch-analytische Arbeit sollen mit Blick auf den ägyptischen Sportunterricht aufgenommen werden, denn nicht nur der ägyptische Sportunterricht, sondern auch die ägyptische Sportdidaktik haben mit drei zentralen Problemen zu kämpfen:

- Ein erstes Defizit - vergleicht man es beispielsweise mit deutschen Standards - liegt z.B. im Mangel an Schulgebäuden, an einer angemessenen Ausstattung der Bildungsstätten sowie an Unterrichtsmaterialien. Dies gilt gleichermaßen für den ägyptischen Sportunterricht sowie für den Unterricht im Allgemeinen. Dadurch kann das Erreichen der Erziehungsziele gründlich infrage gestellt werden, wenngleich diese Annahme noch nicht empirisch überprüft worden ist.

- Betrachtet man die ägyptischen Lehrpläne, so sind hier als Erziehungsziele lediglich sportartspezifische Inhalte festgeschrieben. Erst in jüngerer Zeit sind mit dem Programm für die Weiterentwicklung des Sportunterrichts (ÄGYPTISCHES MINISTERIUM FÜR ERZIEHUNG UND UNTERRICHT, $1998^{1}$ ) erste Bemühungen erkennbar, die auch die übergreifenden Erziehungsziele im Sportunterricht implementieren wollen. Eine Verbindung zwischen übergreifenden Erziehungszielen und Curricula besteht nicht.

- Obwohl Sportlehrerinnen und Sportlehrer die wichtige Funktion haben, den Schülern die Erziehungsziele zu vermitteln, sind bisher Untersuchungen ausgeblieben, die klären konnten, was der Unterricht nach Meinung der Sportlehrkräfte verwirklichen sollte und was sie selbst während des Unterrichts unternehmen. So besteht eine weitgehende Unsicherheit darüber, was die tatsächliche Umsetzung der Erziehungsziele betrifft. Mit der Analyse der Sportlehrerlnnen kann eine wichtige Erklärungsgrundlage dafür geschaffen werden, ob und wieso Erziehungsziele bei den rezipierenden Schülern ankommen. Weitergehend könnte mit entsprechenden Untersuchungen eine Wissensbasis für Planungen und Anpassungen des Sportunterrichts und für die Planung der Sportlehrerausbildung gelegt werden.

\footnotetext{
${ }^{1}$ Ein Großteil der herangezogenen Literatur ist in arabischer Sprache abgefasst. Um den Überblick zu erleichtern, wird die arabische Literatur am Ende der Literaturliste gesondert aufgeführt.
} 
Um derartige Defizite beheben zu können, sind erste explorative Erhebungen notwendig. Dabei ist es erforderlich, einzelne problematische Phänomene aufzudecken, sie in Zusammenhang zu bringen und in einem weiteren Schritt adaptive Lösungen zu entwickeln. Obwohl sich der ägyptische Sportunterricht mittlerweile als Fach etabliert hat, muss er also grundlegend hinterfragt, differenziert ausgeleuchtet und weiterentwickelt werden. Eine wichtige Zielrichtung liegt dabei in der Klärung der Frage, inwieweit die übergreifenden Ziele durch angewandte Unterrichtsverfahren und unter Berücksichtigung der Rahmenbedingungen erreicht werden können.

Dabei sind Erkenntnisse der deutschen Sportdidaktik allerdings nur bedingt übertragbar. Während die „außerordentliche Beliebigkeit in Themenstellung, Problemperspektiven und Grundlagenverständnis“ (vgl. BECKERS, 1987, 241) im deutschen Sprachraum eine einheitliche bzw. - wie BALZ $(1997,10)$ dies ausdrückt eine „aussichtsreiche fachdidaktische Basis“ erschweren, so ist dies in Ägypten kaum der Fall. Gegenüber der deutschen Sportdidaktik mit zumindest vier unterschiedliche Auffassungen (konservative Position des Sportartenlernens, pragmatische Position der Handlungsfähigkeit, dialogische Position der Bewegungserziehung und fundamentale Position des Spaßerlebens; siehe im Überblick: BALZ 2000), beschäftigt sich die ägyptische Sportpädagogik bisher lediglich mit einer Perspektive, nämlich der des Sportartenlernens. Damit ist zwar eine einheitliche Basis gegeben, jedoch kann dadurch kaum das Problem der Implementierung fachübergreifender Erziehungsziele gelöst werden. Zu einer Klärung der Frage, wie nicht nur der ägyptische Sportunterricht, sondern vielmehr auch die ägyptische Sportpädagogik weiterentwickelt werden kann, scheint es hilfreich, mit der vorliegenden Arbeit drei wesentliche Analyseeinheiten in den Blick zu nehmen:

1. Einerseits ist eine Strukturanalyse des Problemfeldes „Ägyptischer Sportunterricht“ vorzunehmen. SCHULZ (1972) schlägt dazu ein Analysemuster anhand von sechs Strukturmomenten vor. Dazu gehören sozial-kulturelle Voraussetzungen, anthropogene Voraussetzungen, Intentionen, Themen, Methoden und Medien. Da sich der Sportunterricht jedoch als Ganzes einer umfassenden Analyse entzieht (vgl. BECKER 1998, 13), ist es demnach „wenig hilfreich, den Unterricht als Ganzes analysieren zu wollen.“ Deshalb müssen Schwerpunkte gesetzt werden und so sind zunächst zentrale Aspekte des ägyptischen Sport- 
unterrichts aufzugreifen. Auf einer abstrakten Reflexionsebene interessieren nun primär folgende Fragen: Wie sehen die Rahmenbedingungen des ägyptischen Sportunterrichtes aus und welche Erziehungsziele werden verfolgt? Welche methodischen Richtlinien und Inhalte sind erkennbar und schließlich: Wie hängen diese Strukturbedingungen zusammen und wo liegen die besonderen Probleme und Schwierigkeiten? Die Strukturanalyse steht unter der Leitfrage: „Welche Situation haben wir vor uns?“

2. Andererseits sind diese Abstrakta einer empirischen Prüfung zugänglich zu machen. Eine wesentliche Voraussetzung für einen erfolgreichen Sportunterricht sind Einstellungen und Handlungsweisen der Lehrer sowie situative Bedingungen; gleichsam liegen hier seine Grenzen (vgl. BECKER 1998). Daher ergeben sich für die weitere Analyse folgende Fragestellungen:

- Inwiefern akzeptiert der Sportlehrer ${ }^{2}$ die einzelnen, allgemeinen Bildungsziele? Sind diese in seiner jeweiligen Wahrnehmung wichtig für seinen Sportunterricht und welche Rahmenbedingungen betrachtet er als erforderlich für seinen Unterricht?

- In einem zweiten Schritt soll aber auch die alltägliche Unterrichtspraxis als Vergleichsmaßstab herangezogen werden. Dabei ist zu fragen, welche Bildungsziele aktiv verfolgt werden. Da hier in den einzelnen Sportarten Unterschiede zu vermuten sind, werden sie punktuell einzeln analysiert.

- Zusätzlich werden Momente der Methodik und der Bewertungspraxis in verschiedenen Sportarten einbezogen.

- Schließlich werden die tatsächlich vorliegenden Rahmenbedingungen analysiert, da sie den Sportunterricht limitieren oder fördern können.

3. Von dieser umfangreichen ersten Analyse sollten Entwicklungsimpulse für die zukünftige Planung - und hier insbesondere die Präzisierung von Curricula des Sportunterrichts ausgehen. Zwar können schon durch diese erste anvisierte Bestandsaufnahme einige Aussagen zur Verbesserung des Systems getroffen werden, jedoch sind insbesondere vor dem Hintergrund problematischer kausaler Annahmen weitere Untersuchungen notwendig. Die vorliegende Ar-

${ }^{2}$ Zur Vereinfachung der Lesbarkeit wird im Folgenden nur noch die maskuline Form gebraucht. Sofern dies also nicht anders kenntlich gemacht ist, gelten die Ausführungen in gleicher Weise auch für Sportlehrerinnen, Schülerinnen usw. 
beit versteht sich daher als erste überblickende Exploration, die weitere Analyseperspektiven eröffnen soll. Dabei soll sie insbesondere Mängel und Hindernisse aufdecken, die den Sportunterricht in seinen Zielsetzungen unterwandern - auch im Hinblick auf Lehrmethoden, Lehrinhalte und die zugrunde liegende Ausbildung der Sportlehrer.

Zur Aufarbeitung der oben aufgeworfenen Fragen und Analyseschwerpunkte ist die vorliegende Schrift in sieben Kapitel unterteilt. In Kapitel 2 werden zunächst die übergreifenden Bedingungen des ägyptischen Kulturraumes i.S. von Voraussetzungen des Sportunterrichts betrachtet. Hier werden also relevante Komplexe dargestellt, die sich - teilweise tief verwurzelt in der historischen Entwicklung - auf den Sportunterricht auswirken sollten: Geographische, politische und volkswirtschaftliche Bedingungen, kulturelle Bedingungen wie der Einfluss von Islam und der Familie auf den Sport, das außerschulische Sportsystem und schließlich die Struktur des Schulsystems.

Daraufhin wird der Lehrplan des Sportunterrichts dargestellt (Kapitel 3). Dieser beinhaltet aber auch Empfehlungen für den Schulsport und die Lehrerausbildung. Als Teil des Bildungssystems interessieren hier v.a. die Aufgaben des Sportlehrers, die Erziehungsziele, Inhalte und Methodik, Rahmenbedingungen und Hilfsmittel und schließlich die Sportlehrerausbildung.

Nach einer eingehenden Beschreibung der daraus resultierenden empirischen Analyse unter methodischen Aspekten (Kapitel 4) folgen im fünften Kapitel deren Ergebnisse und Diskussion. Hauptaugenmerk liegt dabei auf den Zielsetzungen, Formen und Inhalten des Sportunterrichts, den Sportlehrerinnen und Sportlehrern heutzutage in Ägypten anwenden wollen und anwenden. Auf dieser Grundlage und vor dem Hintergrund der Rahmenbedingungen wird ausgearbeitet, welche Konsequenzen sich daraus im Hinblick auf eine Optimierung des ägyptischen Sportunterrichts sowie auf die Ausbildung ergeben, sodass Erziehungsziele zukünftig möglichst weitgehend umgesetzt werden. Unter Berücksichtigung der ägyptischen wie deutschen Literatur wird diskutiert, wie weitergehende Fragestellungen der ägyptischen Sportpädagogik aussehen können, um diese Annahmen zu präzisieren und zu untermauern (Kapitel 6). Schließlich werden die Ergebnisse in Kapitel 7 zusammengefasst. 


\section{Aspekte der ägyptischen Kultur und des Sports}

Bei der Betrachtung und Analyse der meisten sozialen Gegebenheiten oder Phänomene lässt sich kaum isoliert vorgehen. Dies trifft insbesondere auch auf Unterrichtsfächer zu, denn sie sind wie erwähnt zunächst einmal eingebettet in ein Bildungssystem und dieses wiederum in ein Gesellschaftssystem. Wollen wir in unserem Fall nun den Sportunterricht in Ägypten verstehen und daraufhin analysieren, so wird es erforderlich, zunächst solche Rahmenbedingungen zu beleuchten.

In diesem Kapitel sollen daher die gesellschaftlichen Bedingungen und das Schulsystem überblicksartig dargestellt werden. Das Besondere am Sportunterricht ist, dass er darüber hinaus auch vom Sportsystem beeinflusst ist. Er befindet sich gewissermaßen in einem Schnittfeld zweier wesentlicher Hintergrundsysteme. Im Unterschied zu anderen Unterrichtsfächern wie z.B. Mathematik ist der Sportunterricht in seiner Bedeutung letztlich auch vielschichtiger, denn der Sport selbst besitzt eine hohe gesellschaftliche Bedeutung, oder anders: Es besteht ein starkes öffentliches Interesse am Sport. Daher wird auch das Sportsystem in diesem Kapitel dargestellt. Erst vor diesem Hintergrund lässt sich der Sportunterricht adäquat untersuchen und letztlich Empfehlungen für Verbesserungen ableiten.

Ein möglicher Weg zu einem vertieften Verständnis - auch im Hinblick auf die gesellschaftliche Einbettung - liegt in der Aufdeckung der Wurzeln. Anhand der wichtigsten geschichtlichen Stationen werden daher zu Beginn der beiden großen Abschnitte zum ägyptischen Sport- und zum ägyptischen Schulsystem bis in die Gegenwart nachgezeichnet und dabei v.a. die übergeordneten Ziele, die teilweise tradiert sind, in den Blick genommen ${ }^{3}$. Vorangestellt seien jedoch die derzeitigen volkswirtschaftlichen und kulturellen Randbedingungen.

${ }^{3}$ Eine solche Entwicklungsperspektive scheint deswegen angebracht, weil - wie dies schon LEBERT $(1982,1 \mathrm{f})$ bei seiner Untersuchung zum Sport in Ghana feststellte - „die Quellenlage zum Sport in Entwicklungsländern sehr ungünstig und der Umfang an Sekundärliteratur sehr gering ist." 


\subsection{Geographische, politische und volkswirtschaftliche Bedin- gungen}

Von der Gesamtfläche Ägyptens von etwa einer Million Quadratkilometern ist nur ein kleiner Teil bewohnbar bzw. landwirtschaftlich nutzbar. Ein Großteil der Einwohner lebt in Ballungsräumen, beispielsweise in der Hauptstadt Kairo allein ca. acht Millionen. Dies ist auf die besondere geographische Lage zurückzuführen (vgl. dazu KLOSTERMEIER, 1992/93; RAUCH, 1995; SEMSEK, 1995): Zum Einen sind 95\% des Staatsgebietes im subtropischen Trockengürtel Wüste zum Anderen durchzieht der Nil das Land und hinterlässt einen 5-15 km breiten, fruchtbaren Landstreifen. Insgesamt sind damit nur $4 \%$ des Landes urbar. Von Kairo an nach Norden verzweigt sich der Strom in einer der fruchtbarsten und dichtbesiedeltsten Kulturoasen der Erde (s.u.). Während des Winters bringen die Mittelmeerstürme dem Delta ergiebige Niederschläge und Tiefsttemperaturen bis unter 5 Grad Celsius. In Kairo dagegen gibt es pro Jahr nur 4 bis 6 Regentage. Der Hochsommer lässt die Quecksilbersäule bis auf 50 Grad Celsius ansteigen; sobald aber die Sonne untergegangen ist, kühlt es merklich ab. Hauptsächlich im Frühjahr weht der Chamsin, ein heißer Wüstenwind, der Sand- und Staubmassen aufwirbelt und vor sich hertreibt.

Das Wetter übt einen Einfluss auf die Ausrichtung Ägyptens auf bestimmte Sportarten aus (vgl. EL CHOULI 1996b). Die Statistik zeigt, dass etwa 56\% der ägyptischen Studenten Ballspiele wie Basketball, Fußball und Handball betreiben. Solche Sportarten können in offenen Stadien gespielt werden, die wegen des gemäBigten Wetters in Ägypten reichlich vorhanden sind. Mit dem Wetter ist auch die Errichtung der beleuchteten Stadien für die Nacht verbunden, weil die Temperatur an Sommertagen in Ägypten sehr hoch ist. Man versucht, durch nächtliche Sportveranstaltungen eine große Zuschauerzahl anzuziehen und viele Tagesspiele nachzuholen. 
Politisch gesehen ist Ägypten eine präsidiale Republik, die in 27 Verwaltungsbezirke $^{4}$ unterteilt ist (siehe auch zum Folgenden SEMSEK 1995). Die durch Volkswahl nominierte Nationalversammlung wählt den Staatspräsidenten. Er ernennt den Ministerpräsidenten und die Kabinettsmitglieder, zu denen auch ein Sportminister und ein Bildungsminister gehören. Das Land wird gemäß der Verfassung von 1971 als ein sozialistischer arabischer Staat mit dem Islam als Staatsreligion regiert (über 90\% der Einwohner Ägyptens sind sunnitische Muslime und etwa 10\% koptische Christen). Besonderer Wert wird auf die Chancengleichheit und die soziale Solidarität gelegt. Seit 1977 besteht in Ägypten das Mehrparteiensystem (zwischen 1961 und 1977 war die einzige zugelassene Partei die Arabische Sozialistische Union - ASU). Die Amtssprache von Ägypten ist arabisch, die Umgangssprache ist der ägyptisch-arabische Dialekt, der von fast der gesamten Bevölkerung gesprochen wird.

Die Arabische Republik Ägypten ist heute mit gut 74 Millionen Einwohnern (bei einer jährlichen Zuwachsrate von etwas über $2 \%$ in den letzten Jahren) das bevölkerungsreichste Land im arabischen Raum (vgl. ZENTRALES AMT FÜR ÖFFENTLICHE ERHEBUNGEN UND STATISTIK, 2001; CIA, 2004). Letztgenannte Statistik zeigt, dass die schwersten Probleme des Landes in der schwierigen Wirtschaftslage liegen. Bei etwa 20 Mio. arbeitenden Menschen hat das Land eine Arbeitslosenrate von derzeit 12\%; bei einer Inflationsrate von 4,3\% leben $23 \%$ der Bevölkerung unterhalb der Armutsgrenze. In der Nilzone (s.o.) leben ca. 60 Mio. Ägypter, womit die Nilzone zu einer der am dichtesten besiedelten Gebiete der Erde zählt.

Etwa 70 \% der Bevölkerung sind Fellachen, traditionelle Bauern. Hinzu kommen die Beduinen der Wüste, nomadisch lebende Hirtenstämme und die Minderheit der Nubier im mittleren Delta. Obwohl der Grad der Verstädterung im Jahre 1997 etwa $45 \%$ betrug, waren noch etwa $42 \%$ der Bevölkerung in der Landwirtschaft tätig, in der hauptsächlich Zuckerrohr und Baumwolle abgebaut werden (vgl. ZENTRALES AMT FÜR ÖFFENTLICHE ERHEBUNGEN UND STATISTIK, 2001).

\footnotetext{
${ }^{4}$ El Dakahlia, El Bahr el Ahmar, El Buhayrah, el Fayyum, El Gharbiyah, El Iskandariyah, El Ismailiyah, El Gizah, El Minufiyah, El Minya, El Qahirah (Kairo), El Qalyubiyah, El Wadi el Gadid, El Sharqiyah, El Suwais, Aswan, Asyut, Bani Suwayf, Bur Said, Dumyat, Ganub Sina, Kafr el Shaykh, Matruh, Qina, Shamal Sina, Suhaj, Luxor.
} 
Durch Bewässerung werden fünf Ernten in zwei Jahren ermöglicht, jedoch nimmt die Bodenversalzung zu und der fruchtbare Nilschlamm wird im Nassersee zurückgehalten, was den Einsatz von Kunstdünger erforderlich macht.

Trotz politischer Erfolge der letzten Präsidenten konnte die Zunahme der Auslandsverschuldung (jährlich etwa 20 Mrd. \$; vgl. ZENTRALES AMT FÜR ÖFFENTLICHE ERHEBUNGEN UND STATISTIK, 2001) nicht verhindert werden. Unterstützung bekommt das Land am Nil aber vor allem von den USA (nach ALSHARQ ALAWSAT, 2004 neuerdings 300 Mio. \$ jährlich) und von der Bundesrepublik Deutschland; beide sind auch wichtige Außenhandelspartner. Seit Beginn der 90er Jahre steht Ägypten an erster Stelle der Empfängerländer deutscher Entwicklungshilfe. Die Gelder flossen vor allem in die Sicherung der Energieversorgung, in den Umweltschutz und dienten der Produktionssteigerung in Industrie und Landwirtschaft.

Trotz der ständigen Ertragssteigerung in der Landwirtschaft ist Ägypten aber nicht in der Lage, sich selbst zu versorgen (vgl. schon SEMSEK, 1995). Rund 75\% der jährlich benötigten neun Mio. Tonnen Getreide sind Importe aus Australien, den USA und Kanada. Jedoch verderben aufgrund von höchst mangelhafter Lagerung jedes Jahr Tausende von Tonnen Getreide. Ägypten hat aber auch viele Bodenschätze, vor allem Erdöl. Trotz einem steigenden Energiebedarf ist das Land in der Lage, noch $50 \%$ seines geförderten Erdöls zu exportieren, wodurch notwendige Devise eingenommen werden können. Ein stark gewinnbringender Sektor ist die Tourismusbranche, auf die Ägypten angewiesen ist. Der Fremdenverkehr als großer Devisenbringer spielt eine bedeutende Rolle, hat jedoch durch Anschläge islamischer Fundamentalisten einen herben Einbruch erlitten. Auch die gegenwärtigen Krisen im Nahen Osten wirken nachteilig auf den Tourismus in Ägypten.

Die schon länger anhaltende schlechte Wirtschaftslage führte zu wirtschaftlichen Neuerungen, wie z.B. der Übereinkunft über Handel und Tarife zur Liberalisierung der wirtschaftlichen Rahmenbedingungen in Bezug auf die ausländische Wirtschaft (GATT). In der Folge wirkte sich die wirtschaftliche Entwicklung auf den öffentlichen Sport aus, darunter die Sportorganisationen, weil diese die ebenfalls neuen Strukturen übernahmen und von den wirtschaftlichen Erfolgen durch die Mitglieder des GATT profitierten. Der öffentliche Sport wird also von den wirtschaftlichen Bedingungen beeinflusst (vgl. ABD EL HAMID, 1999, 79f): Die Sportverbände litten sehr unter der finanziellen Krise, die durch überhöhte Staatsaus- 
gaben verursacht wurde. In den Jahren 1992 bis 1998 war die finanzielle Unterstützung durch die Regierung, die meist die wesentliche Einnahmequelle für die Verbände darstellte, eingefroren, die Ausgaben hingegen stiegen durch die Inflation an. Dadurch wurden die Verbände gezwungen, sich nach anderen Geldquellen umzusehen. Durch diese sollte das Niveau des Sportangebots gehoben und die Dienstleistungen verbessert werden.

\subsection{Allgemeine kulturelle Bedingungen}

\subsubsection{Islam und Sport}

Wie oben erwähnt, bekennen sich mehr als 90 \% der ägyptischen Bevölkerung zum Islam. Ägypten ist als „islamischer Staat“ zu bezeichnen, denn der erste Artikel der Ägyptischen Verfassung legt fest, dass bei der Abfassung von Gesetzen Rücksicht auf den Islam zu nehmen ist. Insofern beeinflusst er als religiöse Leitkultur letztlich alle gesellschaftlichen Gruppierungen, zu denen auch Sportorganisationen und insbesondere staatliche Sportinstitutionen gehören (vgl. EL CHOULI, 1995, 14); er wird zum bestimmenden Einfluss auf die Handlungsweisen sowohl der Menschen untereinander als auch in Bezug auf den Umgang mit Gütern und Dienstleistungen.

Allerdings ist nicht auf den ersten Blick festzustellen, welches Verhältnis zwischen Islam und Sport besteht. Da die Bildungsziele aber wie bereits erwähnt in eine solche Kultur eingebettet, ja gar durch sie entstanden sind, ist das grundsätzliche Verhältnis aufzuarbeiten. Ich werde daher in diesem Kapitel zunächst die Grundlagen des Islam darstellen und daraufhin die Bewegungskultur bzw. das Bewegungsverständnis aus Sicht des Islam verdeutlichen. Auf dieser Basis werde ich dann versuchen, die historische Entwicklung der Bewegungskultur im Allgemeinen und der Sporterziehung im Besondern bis heute nachzuzeichnen. 
Um Missverständnissen vorzubeugen, sei an dieser Stelle jedoch eine Vorbemerkung angebracht: Der Islam wird in der sog. „aufgeklärten Welt“ bzw. der westeuropäischen wie nordamerikanischen Kultur oftmals pauschal negativ bewertet. Medienberichte über fundamentalistische Strömungen ${ }^{5}$ oder Bilder von verschleierten Frauen geben den Menschen westlicher Kulturen häufiger das Gefühl, dass es sich beim Islam um eine rückschrittliche, antiwestliche oder gar gefährliche Bewegung handelt. Mit Ende des „Kalten Krieges“ (der Ost-West-Konflikt) und aufgrund der Tatsache, dass der Islam die zweitgrößte Weltreligion (mit über einer Milliarde Gläubigen) ist, wird in letzter Zeit des öfteren ein neuer, weltumspannender Konflikt zwischen der westlichen und der islamischen Welt beschrieben oder gar von einem "Krieg der Zivilisationen" gesprochen (vgl. z.B. SCHOLL-LATOUR, 2002): Christliche, fortschrittliche und wirtschaftlich überlegene vs. islamische, rückständige und wirtschaftlich unterlegene Welt. Doch der Fundamentalismus ist nur eine Seite des facettenreichen Islams. In seinen Ausprägungsformen kann der Fundamentalismus als eine Folge der wirtschaftlichen Not angesehen werden (vgl. TIBI, 1985, 67f). So wie auch im Westen bei schlechter konjunktureller Lage nationalistische Strömungen verstärkt auftreten, so tritt nunmehr in islamischen Ländern verstärkt fundamentalistisch-nationalistisches Gedankengut auf, das sich ursprünglich gegen die wirtschaftliche Not bzw. die "kulturelle Ausbeutung" oder Abhängigkeit von westlichen Ländern wendet. Insofern ist die jeweilige Ausdrucksform des Islams nicht nur auf die religiösen Inhalte, sondern vielmehr auch auf die säkularen Gegebenheiten zurückzuführen, die sich von Land zu Land und von städtischen zu ländlichen Regionen unterscheiden. Die jeweiligen Verknüpfungen von Traditionen oder politischen Strömungen mit dem Islam sind nicht mit inm selbst zu verwechseln.

\footnotetext{
${ }^{5}$ Insbesondere aus den Hauptverbreitungsgebieten des Islam im Vorderen Orient (z.B. Türkei, Iran, Irak und Vereinigte Arabische Emirate, Saudi-Arabien), aus nordafrikanischen Ländern (z.B. Ägypten, Sudan, Algerien und Tunesien) und aus ostasiatischen Ländern wie Pakistan, Malaysia und Indonesien, daneben Indien und China.
} 


\subsubsection{Grundlagen des Islams}

Entstanden ist der Islam im 7.Jh. n.Chr. Die Araber waren damals Beduinen, d.h. Nomaden, die mit ihren Zelten, Ziegen, Schafen und Kamelen auf der Suche nach Weideplätzen für ihre Tiere umherzogen. Ein Teil der Araber bestritt seinen Lebensunterhalt durch Handel (aber auch durch Überfälle auf Handelskarawanen zumindest zeitweise). Vor diesem Hintergrund geht TIBI (1985, 24f) sogar soweit, dass er den Islam als eine den Bedürfnissen der Araber entsprungene Ideologie beschreibt.

Der Verkünder des Islams, der Prophet Mohammed, erblickte um das Jahr 570 n.Ch. in Mekka (heute in Saudi-Arabien) das Licht der Welt. Nach dem frühen Tod seiner Eltern verbrachte er seine Kindheit und Jugend bei seinem Onkel Abu Talib und heiratete mit 25 Jahren die 40jährige Handelsunternehmerin Chadiga. Nach der Überlieferung erhielt Mohammed mit vierzig Jahren die Offenbarung des Wortes Gottes durch den Engel Gabriel in der Höhle Hira' in der Nähe von Mekka. Dorthin zog er sich immer zur Meditation zurück, um Abstand vom „ethischen Verfall" (vor allem Götzenverehrung) in seiner Umgebung zu nehmen, denn die Araber waren damals Polytheisten. Diese Zeit wird im Arabischen die Zeit der Unwissenheit genannt. Zunächst stieß er mit seinen Predigten Anweisungen und Ermahnungen, die er nach seiner Berufung hielt, nicht auf Wohlgefallen, sondern konnte nur eine kleine Gemeinde um sich scharen, die hauptsächlich aus seinen Verwandten bestand. Aufgrund zunehmender Repressalien seitens der Bevölkerung floh er mit seiner Gemeinde zwölf Jahre nach der Verkündung seiner islamischen Offenbarung im Jahre 622 nach Jathrib (später umbenannt in El Medina, die Stadt), einer weiter nördlich liegenden Stadt, die hauptsächlich von Juden bewohnt war. Die Higra (Auswanderung Mohammads) gilt als der Beginn der islamischen Zeitrechnung. In Medina, wo auch die erste Moschee gebaut wurde, verkündete er seine Lehre und durch kriegerische Eroberungen (im Jahre 630 Mekka) stieg die Zahl seiner Anhänger. Er festigte seine Führerschaft durch weitere Verkündigungen von Offenbarungen, die er zu aktuellen Fragen der Gemeinschaft erhielt. Das erklärte Ziel war dabei primär die Reinigung der Städte von Götzenbildern und -statuen. In Mekka ließ er die Kaaba (den schwarzen Stein) stehen, da sie von einem der vom Islam anerkannten Propheten, nämlich Ibrahim, aufgebaut 
wurde und erklärte es zum Heiligtum und Wallfahrtsort der Muslime. Bis zu seinem Tod im Jahre 632 in Mekka, wo auch seine Grabstätte liegt, hatte Mohammed es geschafft, alle Stämme der arabischen Halbinsel zu einen und zum Islam zu bekehren (vgl. EL ASKALANI, 1996, 158).

Der Islam versteht sich als die letzte übermittelte Offenbarung Gottes. Somit ist sie „die abschließende Wahrheit, die für alle Zeiten, alle Religionen und die gesamte Menschheit gelte“; Mohammed, der laut dem Koran ${ }^{6}$ die letzte Offenbarung Gottes verkündet hat, wird als "Siegel aller Propheten" bezeichnet (vgl. EL ASKALANI, 1996, 164). Mohammeds empfangene Offenbarung ist dementsprechend die wichtigste Grundlage des Islam. Der Koran ist in arabischer Sprache verfasst und darf auch nur in der arabischen Sprache unterrichtet werden. Das Leben des strengen Muslims richtet sich nach den Vorschriften des Koran und der Hadith („Überlieferung“: Sammlung der Aussprüche und Taten des Propheten). Letztere Anweisungen liegen in Ausarbeitungen verschiedener ,Schulen' als Richtlinien des alltäglichen Lebens und der islamischen Rechtsprechung vor.

Aus dem arabischen übersetzt bedeutet nun das Wort Islam "Selbstunterwerfung, Hingabe". Gemeint ist die völlige Unterwerfung unter den göttlichen Willen und die heiligen Schriften. Somit beruht der Islam auf der kompromisslosen Anerkennung der göttlichen Einzigartigkeit. Die von ihm geschaffenen Wesen haben eine beschränkte und bedingte Existenz. Nach der Lehre des Islams ist der Mensch nie allein, denn die göttlichen Augen ruhen immer auf ihm. Er ist jederzeit in „Begleitung eines Schöpfers, der barmherzig und weise ist" (EL CHOULI, 1995, 34).

Man kann aus dieser grundlegenden Sichtweise der Beziehung zwischen Gott und Mensch (Beschützer - Diener) ableiten, dass die Wertschätzung des Menschen nur gering ist. Seine Existenz beruht darauf, Gott zu dienen. Er ist kein selbständiges Wesen, denn seine Entscheidungen sollten immer in Einklang mit dem in der heiligen Schrift niedergeschrieben göttlichen Willen getroffen werden. Im Koran sind nun folgende zentralen Glaubenssätze und religiöse Grundpflichten enthalten:

\footnotetext{
${ }^{6}$ Der Koran (arabisch El Quran, Vortrag, Liturgie) ist das heilige Buch des Islams. Es umfasst 114 Suren (Abschnitte) und 6666 Verse, die in den rund zwei Jahrzehnten nach dem Tode Mohammeds schriftlich aufgezeichnet und ihrer Länge nach aneinander gereiht wurde; die längeren Suren stehen am Anfang und jede Sure fängt mit den Worten „im Namen Allahs, des Gnädigen und des Barmherzigen" an.
} 
1. Glauben an einen Gott.

2. Glauben an die Engel.

3. Glauben an den Koran als göttliche Offenbarung.

4. Glauben an die Propheten Gottes, deren letzter und erhabenster Mohammed ist.

5. Glauben an ein jüngstes Gericht (Verantwortung für die irdischen Taten im Jenseits).

6. Glauben an die Vorsehung.

Im Unterschied zum Christentum bei dem die Taufe ein zentrales Glaubensbekenntnis darstellt, gibt es einen solchen Aufnahmeritus im Islam nicht. Entweder man wird hineingeboren oder man tritt in die heilige Gemeinschaft des Islams ein, indem man ehrlich und aufrichtig das Glaubensbekenntnis (Schahada) ablegt: „La ilaha illa'llahu, Muhammad rasulu'llahi“ (Es gibt keinen Gott außer dem (einen) Gott, und Mohammad ist der Gesandte Gottes).

Das ebenfalls grundlegende Ritualgebet ist als ein Akt der Verehrung und Unterordnung unter den Willen des einzigen Gottes zu verstehen. In ihm werden hauptsächlich Koranstellen rezitiert, die in besonders vorgeschriebenen Körperhaltungen ausgesprochen werden. Wichtig ist, dass man dies in Richtung Mekka gewandt tut und an einem reinen Platz ausführt. Das Reinheitsgebot schreibt vor, dass dem Gebet eine rituelle Waschung vorausgeht (vgl. TIBI, 1985, 32f).

Der Ramadan (einmal im Jahr für vier Wochen zu fasten) gehört ebenfalls zu den heiligen Glaubenspflichten des Islam. Er enthält die Aufforderung, von Sonnenaufgang bis Sonnenuntergang nichts zu essen, zu trinken oder zu rauchen. Einige Wissenschaftler sehen darin ein Hemmnis für die Sportausübung. So kann das Fasten beispielsweise unwirksam werden, wenn beim Schwimmen der Mund mit Wasser in Berührung kommt. Da die Periode des Ramadan durch alle Jahreszeiten wandert, ist die körperliche Belastung besonders groß, wenn er in den Sommer fällt und auch in der orientalischen Hitze zwischen frühem Morgenlicht und Sonnenuntergang kein Tropfen Wasser gestattet ist.

Zusammenfassend enthält der Islam fünf Grundpflichten, zu denen neben den drei genannten noch Almosengabe und nach Möglichkeit noch eine Pilgerfahrt nach Mekka gehört. Folgende Grundpflichten werden auch als die "fünf Säulen" des Islams bezeichnet: 
1. Das Glaubensbekenntnis an einen einzigen Gott (Schahada) und seinen Propheten Mohammed.

2. fünfmaliges Gebet pro Tag (Salaat).

3. Fasten im Ramadan (Siyam).

4. Almosenabgabe (Zakat); 1/40 seines Einkommens.

5. Pilgerfahrt nach Mekka (Hagg), allerdings nur für diejenigen, die körperlich und finanziell dazu in der Lage sind.

Inhaltlich behandelt der Koran ebenso religiöse wie auch soziale und politische Fragen. In der Praxis bedeutet das, dass der Islam, wenn man nach seinen Regeln lebt, eine extreme Kontrollfunktion ausübt, welche die Gläubigen mit einem Mantel sozialer Kontrolle umgibt. Der Islam hat auf alle möglichen gesellschaftlichen Fragen eine Antwort, wobei die Antworten verschieden ausfallen können, da manche Koranstellen vieldeutig sind. Dies betrifft auch den Sport bzw. die Bewegungskultur.

\subsubsection{Islamische Aussagen zu Bewegung, Spiel und Sport}

Im Koran selbst sind keine ausdrücklichen Stellen zu finden, die sich mit der körperlichen Betätigung beschäftigen. Wenn man die Haltung des Islams zu Spiel und Freude finden will, muss man die Aussprüche des Propheten betrachten, die als Ahadith gesammelt und überliefert sind und deren Bedeutung gegenüber dem Koran letztlich nicht zurück stehen. Die Muslime benutzen diese Empfehlungen des Propheten, um ihr Leben danach zu richten, von den Ulama werden sie als eine Quelle islamischen Rechts genutzt.

Der Prophet selbst erteilte Ratschläge und ermunterte dazu, mehrere Sportarten auszuüben. Als beispielhaftes Vorbild für alle Muslime übte er mehrere Sportarten öffentlich aus (vgl. ZARGHAMI, 1997, 85). Dazu gehörten v.a. Bogenschießen, Ringkampf, Pferdesport und Wettlaufen. Solange der Gläubige seinen Lebenszweck und die religiösen Pflichten nicht vergaß, war es nach Meinung des Propheten nicht schädlich, seinem Vergnügen nachzugehen. Die Spiele und Sportarten, die der Prophet empfahl, sollten zwei Zielen dienen; auf der einen Seite der Erholung und auf der anderen Seite dem körperlichen Training, um auf Kriege vorzube- 
reiten. Zur Zeit des Propheten wurden bekanntermaßen folgende Sportarten ausgeübt (vgl. ZARGHAMI, 1997):

\section{Bogenschießen}

Hier handelte es sich um Wettkämpfe mit Pfeil und Bogen. Verboten hat der Prophet, etwas Lebendiges als Übungsziel zu benutzen.

\section{Speerspiele}

Speerdarbietungen wurden sogar in der Moschee vorgeführt, denn die Moschee sollte nicht nur religiösen Zwecken dienen, sondern auch zu weltlichen Zwecken benutzt werden.

\section{Jagen}

Jagen wurde als eine nützliche Sportart gesehen. Verboten war es in zwei Situationen: während der Pilgerfahrt nach Mekka und in den Grenzen des heiligen Bezirks von Mekka.

Mohammed befürwortete auch, dass die Kinder Sport treiben. So wird von Abu Rafi' erzählt, dass er den Propheten fragte, ob die Kinder die gleichen Rechte gegenüber den Eltern hätten wie diese gegenüber ihnen. Der Prophet antwortete ja, das Recht des Kindes gegenüber den Eltern besteht darin, von diesen das Schreiben, Schwimmen und Bogenschießen zu lernen und von innen zu erben (vgl. EL ASKALANI, 1996, 142f).

Nach den Aussagen des Propheten sind neben Wettkämpfen zwischen Männern auch solche zwischen Männern und Frauen erlaubt, solange es sich um die Ehefrauen handelt. Auch die Sportausübung unter Frauen alleine ist unter bestimmten Bedingungen (wie z.B. bestimmte Kleidung) gestattet (vgl. EL ASKALANI, 1996, 212). Der Islam gestattet den Frauen beispielsweise auch, bei Sportveranstaltungen und Wettkämpfen zuzusehen. Im Islam ist das Ausüben von Sport also nicht auf die Kindheit oder ein Geschlecht begrenzt. Dies wird auch durch eine andere prophetische Überlieferung bestätigt (vgl. DARWISH \& EI HAMAHMI, 1997, 290f). Resümierend ist festzustellen, dass Sport und Spiel nicht nur erlaubt (solange es sich nicht um Glücksspiele handelte), sondern sogar erwünscht sind. Im Koran und in den prophetischen Überlieferungen gibt es Hinweise auf sportliche Tätigkeiten, sodass „Klassischer Islam und der Koran (...) keinerlei Abwertung der Leiblichkeit und der Leibesübung [implizieren]“ (vgl. YALDAI, 1986, 39f). Somit ist für 
uns ersichtlich, welche Sportarten bei der Entstehung des Islams betrieben wurden. (vgl. ZARGHAMI, 1997, 79).

\subsubsection{Historische Entwicklung der islamischen Bewegungskultur}

In der Gesellschaft des frühen Islams gab es öfter Sportereignisse, sowohl vor als auch nach dem Hinscheiden des Propheten und während der darauf folgenden Regierungszeit der ersten „vier Rechtgeleiteten Khalifen ${ }^{7 “}$. Die Nachfolger des Propheten folgten seinen Empfehlungen, indem sie Plätze für Training und Wettkampf schufen und auch finanziell herausragende Athleten unterstützten. Doch insgesamt wurden körperliche Kraft, Schnelligkeit und Gewandtheit meist in Zusammenhang mit der Wehrertüchtigung und dem Krieg gegen die Ungläubigen gesehen (vgl. EL CHOULI, 1995, 112). Der Sport fand sowohl bei der Bevölkerung als auch den islamischen Geistlichen, den Ulama', Unterstützung. Es ist wahrscheinlich, dass schon die frühislamischen Araber viele Sportarten getrieben haben, wie z.B. Bogenschießen, Schwimmen, Reiten, Ringen, Laufen, Kraftproben u.v.m (vgl. EL CHOULI, 1995, 176). Viele spätere Autoren nach den Rechtgeleiteten Khalifen wiesen auf die Bedeutung des Sports und der Bildung hin und behandelten wichtige Aspekte, z. B. in welchen Etappen Sportaktivitäten durchzuführen und zu erlernen sind.

Die Orte, an denen vor dem Entstehen von allgemeinbildenden Schulen gelehrt wurde, waren die Moscheen und Wohnungen der Gelehrten. Dies blieb so, bis im Jahr 1805 mit Muhammad Ali ein Osmane die Herrschaft übernahm und seine Macht auf das Heer stütze. Er förderte die Wirtschaft und Bildung, indem er Kader heranzog, die von nach Europa gesandten Gelehrten in Wissenschaft und Politik unterwiesen wurden. Muhammad Ali schuf ein Bildungswesen, das in ein religiöses und weltliches unterteilt war. Letzteres beinhaltete besonders Schreiben, Sprachen (Türkisch und Französisch) und Sport. Gerade der Sport sollte dann dem Heer zugute kommen, denn die spezifischen Bewegungsformen waren Reiterei, Fechten und Ringen. 1831 wurde sogar eine Reitschule gegründet, die dazu diente, das Heer auszubilden und auf den Kampf vorzubereiten - nicht jedoch für die gemeine Bevölkerung. Auf die Sporterziehung der Bevölkerung legten die osmanischen Kolonialherren keinen Wert, denn der Sport hatte sich den Erfordernis-

\footnotetext{
${ }^{7}$ Abu Bakr el Sidiq, Omar ibn el Chattab, Uthman ibn Afan und Ali ibn Abi Talib.
} 
sen des Heeres anzupassen; er diente aber auch dazu, die Schüler durch Leibesübungen auf die militärischen Erfordernisse vorzubereiten (vgl. HUSSEIN, 1973, 33).

In den Jahren 1867 bis 1882 begann ein neuer Abschnitt, der der kulturellen Erneuerung nach einem von den Ägyptern als Niedergang erlebten Zeitalter. Zu jener Zeit herrschte der Khediv Ismael, der Ägypten in allen Bereichen modernisieren wollte, darunter auch den Sport- und Erziehungsbereich. Er schuf ein gestuftes Bildungssystem, Museen, gelehrte Gesellschaften und große öffentliche Bibliotheken. Auch das Interesse an der Sporterziehung an den ägyptischen Schulen nahm zu. Die Schüler erhielten Unterricht in Leibeserziehung durch Laufen, Stockund Seilübungen (vgl. HUSSEIN, 1973, 47).

In den Jahren 1882 bis 1925 folgte die Periode der englischen Besetzung, in denen das Ziel der Bildung die Herausbildung einer Beamtenschicht war. Die Briten bemühten sich, ihr kulturelles System auf ägyptische Gesellschaft zu übertragen und bestimmten den Sport als festes Schulfach, doch mangelte es an der Umsetzung. Sie verbreiteten daneben auch „ihre“ Sportarten, wie Fußball, Tennis, Hockey und Kricket. Der erste gegründete Sportclub im Jahre 1887 war eine Militärschule für Sporterziehung, an der zehn englische Lehrer Unterricht in Sport und Leibeserziehung erteilten (vgl. EL CHOULI, 1995, 183ff).

Nach 1925 begann man, sich mehr Gedanken um die Bildung zu machen und das Wissenschaftsministerium richtete die Leibeserziehung als grundlegendes Unterrichtsfach ein. Es bemühte sich um die Umsetzung, indem es ehemalige englische Soldaten für den Sportunterricht einstellte. Der Unterricht umfasste Gymnastik und Turnen, wozu die nötigen Geräte angeschafft wurden. Das damalige Unterrichtswesen folgte noch sehr dem traditionellen Weg, der auf der bloßen Wissensvermittlung beruhte. Die Schüler lernten nur, um ihre Prüfungen zu bestehen und das Zeugnis zu erhalten. Der Sportunterricht war allerdings mehr ein Zeitvertreib denn Unterrichtsfach, dass spezielle Absichten wie beispielsweise eine gezielte Leistungssteigerung i.S. des Wettkampfsports verfolgte (vgl. EL CHOULI, 1995, 191f). Vielen islamischen Geistlichen und Intellektuellen war schon in alter Zeit der Wert des Sports für die Erziehung nicht verborgen geblieben. In ihren Äußerungen preisen sie die sportlichen Aktivitäten. Der Imam Abu Hamid el Ghazali wies im späten Mittel auf die Bedeutung eines schönen Spiels für Knaben hin, um sich von den Anstrengungen des Bücherstudiums zu erholen. Der Universalgelehrte Ibn Chal- 
dun machte schon in einem Vorwort seiner berühmten Universalenzyklopädie auf den Zusammenhang zwischen körperlicher Konstitution und Ernährung sowie der körperlichen Auseinandersetzung mit der Umwelt aufmerksam. IBN EL QAYIM, EL GAUZIJA hat ein Buch über Sport unter dem Titel „Die Reitkunst“ herausgegeben (vgl. EL GAUZIJA, o. J.).

Um jüngeren Menschen den Sport aus islamischer Sicht zu erläutern, werden ihnen auch heute noch Bilder der Gläubigen in ihrem Lebenslauf gezeigt. Dabei darf nicht vergessen werden, dass die Grundlagen dazu beitragen, die Leiblichkeit der Individuen islamisch zu begründen. Es soll den jungen Menschen die Einstellung Mohammed zu den verschiedenen Arten der Leibesübungen vermittelt werden. Dies hat seinen Hintergrund im Verhältnis des Islams zur Bildung im Allgemeinen, nachdem die Suchen nach Wissen große Bedeutung beigemessen wird und seine größte Wissensquelle der Koran mit seinen Versen, „Wunderzeichen“ ist: „Sind denn die Wissenden und die Unwissenden gleich?“ (DER KORAN, Sure el Zumur, Vers 9). Daneben stehen weitere Aussprüche des Propheten: „Ein Wissenssuchender gilt solange als klug, wie es nicht scheint, dass er nicht wisse“ und „Wer dem Weg folgt und das Wissen erlangt, dem ebnet Gott den Weg zum Himmel“ (vgl. El ASKALANI, 1996, 115f).

So kann man sagen, dass der Islam und der Sport den Einzelnen fördern, indem sie beispielsweise sein Selbstvertrauen stärken. Da der Sport im Islam als Mittel zur Förderung der sozialen Bindung und gesellschaftlichen Erfahrung gilt und da er Situationen und Bedingungen, wie sie das Leben erfordert, vermittelt, kommt ihm grundsätzlich eine hohe Bedeutung zu. Der Mensch kann in einer Sportmannschaft beispielsweise Verantwortung in der Zusammenarbeit mit anderen Menschen tragen lernen. Zudem ruft der Glaube zur gegenseitigen Verbindung, Anerkennung sowie zum Gehorsam und Gefolgschaft gegenüber der Gemeinschaft sowie zu Bescheidenheit und Hilfeleistung gegenüber anderen auf. Diese Faktoren tauchen in nahezu allen Sportbereichen auf, wozu auch der Sportunterricht gehört, dessen Ziele ebenso die allgemeinen Erziehungsziele reflektieren (siehe Kapitel 3.1). Ich meine daher, dass sowohl der Sport als auch der Islam Systeme sind, die die umfassende Erziehung des Menschen zum Ziel haben. Daraus resultiert, dass beide Systeme häufig zusammenwirken und eine starke Wirkung auf das Bildungssystem haben. Besonders deutlich wird dies an der „Azhar“. 
Bis in unsere Zeit stellt die Azhar die wichtigste und größte religiöse und wissenschaftliche Institution der islamischen Welt dar. Sie ist beständig vergrößert und auf weitere Bildungsgebiete ausgedehnt worden. Sie umfasst Grundschule, neuerdings auch Vorschule, Mittel- und Oberschule sowie vielfältige Studiengänge in ihrer Universität. Viele ihrer Studenten kommen aus dem islamischen Ausland.

Die ,Azhar' bietet den Studenten viele Sportarten an, aus der Überzeugung, dass die Sportausübung für die Studenten und zum Erreichen der Erziehungsziele wichtig ist (vgl. AWIS, 1994, 7). Insgesamt treiben 30.000 Studenten und Studentinnen Sport. Folgende Grafik gibt einen Überblick zu verschiedenen Sportangebote, die von den Studenten in ihrer Freizeit genutzt werden (vgl. EL CHOULI, 1995, 179f).

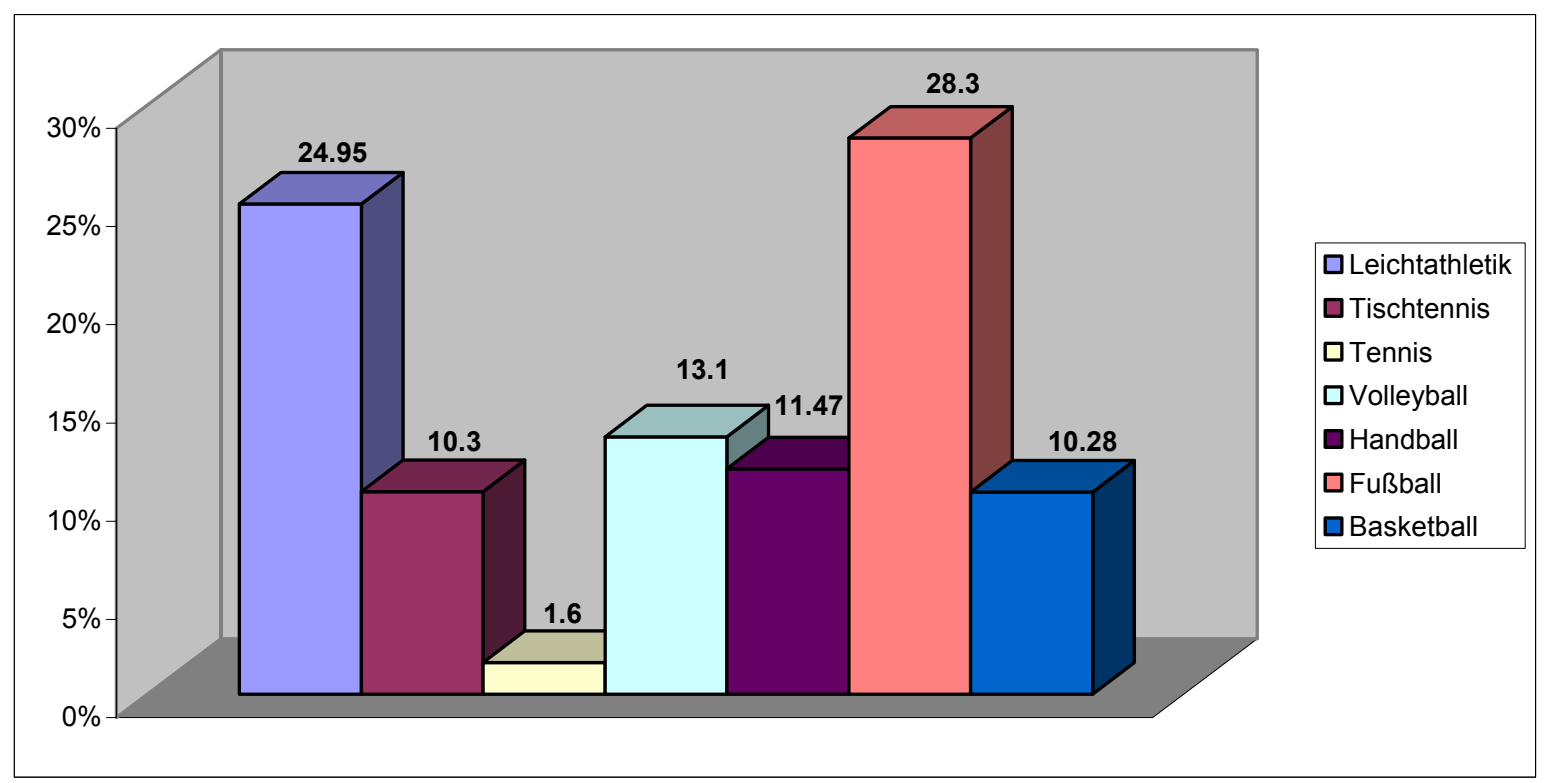

Abbildung 1: Teilnahme von Schülern und Studenten der Azhar an verschiedenen Sportangeboten (Quelle: ZENTRALES AMT FÜR ÖFFENTLICHE ERHEBUNGEN UND STATISTIK 2001).

Anfang der 90er Jahre gründete die Azhar-Institution eine Sportfakultät, um Sportlehrer und Trainer für die Arbeit in religiösen Bildungsinstitutionen gezielt auszubilden. Sie arbeitet auch mit dem Ministerium für Jugend und Sport sowie Sportverbänden zusammen. So nahm die Azhar-Institution während der Afrikanischen Spiele im Jahr 1991 über 1500 Sportler, in ihren Studentenwohnheimen auf (vgl. DER HOHE RAT FÜR JUGEND UND SPORT, 1997, 489f). Neben der Organisation und Durchführung von nach Geschlechtern getrennten Wettkämpfen stellt sie 
während der vorlesungsfreien Zeit ein Sportprogramm für alle, d.h. besonders auch für die Anwohner der Azhar, bereit.

\subsubsection{Familie und Sport}

Die Familie ist die erste Sozialisationsinstanz im Leben eines Kindes. Sie hat daher einen wichtigen Einfluss auf viele Lebensbereiche, so auch auf den Sport. Allerdings sind innerhalb der ägyptischen Sportwissenschaft Untersuchungen ausgeblieben, die diesen Zusammenhang zu klären vermochten.

Im deutschsprachigen Raum liefert HEINEMANN (1980, 167ff) einen Rückblick auf Untersuchungen, aus denen hervorgeht, dass es einen Zusammenhang zwischen der sportlichen Tätigkeit der Eltern (Einstellung zum Sport) und dem Sportinteresse der Kinder gibt. Beispielsweise fand ARTUS (1974, 81), dass 55\% der Väter und ca. 46\% von Mütter von Sporttreibenden Jugendlichen für das Sporttreiben sind und auch selbst Sport treiben. Aus diesen Ausführungen ersieht man, dass die Familie direkten Einfluss auf das Verhalten der Jugendlichen beim Sport hat. Die Familie prägte genauer das grundlegende Sportinteresse, während die gleichaltrigen Kameraden (Peer Group) stärker die Richtung des Sportinteresses bestimmte.

Alle aufgeführten Untersuchungen wurden in industrialisierten Ländern durchgeführt und es ist nun davon auszugehen, dass sie hier verallgemeinerbar sind. Da aber einerseits Aussagen zu einer arabischen bzw. islamischen Gesellschaft getroffen werden sollen und andererseits keine empirischen Befunde vorliegen, soll hier der Versuch unternommen werden, erste vorläufige Hypothesen herauszuarbeiten.

Eine arabische bzw. ägyptische Familie besteht aus Eltern, aus noch nicht verheirateten Kindern und verheirateten Söhnen mit ihren Familien; die verheirateten Töchter ziehen hingegen zu der Familie des Mannes und stehen unter deren Einfluss. Es handelt sich also meist um Großfamilien unter einem Dach.

In der Familie dominiert - legitimiert durch den Koran - der Vater; er bestimmt fast alles und ist Herr der Familie. Über seine Entscheidungen darf nicht diskutieren werden, womit er uneingeschränkte Autorität hat. Er entscheidet in den meisten 
Fällen, beispielsweise über die Studienwahl der Kinder, wer wen heiratet und was im Haushalt neu angeschafft werden soll. Seine Autorität stützt sich auf seine Stellung als Grundbesitzer und auf seine Stellung als arbeitender Ernährer der Familie. So haben die Kinder grundsätzlich kein Recht zum Widerspruch und auch die Söhne haben bis zum Tode ihres Vaters keinen Anspruch auf das Besitztum. Die Stellung der Frau ist ebenfalls im Koran bestimmt: Sie soll sich um Haushalt und Kinder kümmern und dem Mann gehorchen.

Dementsprechend müssen die obigen Aussagen, die aus den deutschen Untersuchungen abgeleitet wurden, zumindest dahingehend relativiert werden, als für den ägyptischen Kulturraum nicht von einem Einfluss der Eltern, sondern vielmehr von einem Einfluss des Vaters auf das Sporttreiben ausgegangen werden kann. Dies ist allerdings noch im Hinblick auf viele Aspekte zu untersuchen. Eine erste wichtige Grundlage wäre beispielsweise schon dahingehend zu legen, als erst einmal geklärt werden müsste, wie viele Ägypter (welchen) Sport treiben und welche Einstellungsstrukturen in dieser Gesellschaft vorliegen.

\subsection{Außerschulisches Sportsystem}

\subsubsection{Historische Entwicklung}

Anhand des Jahrbuches des ÄGYPTISCHEN MINISTERIUMS FÜR JUGEND UND SPORT (2001, 39f) ist festzustellen, dass ägyptische Sporthistoriker einen Einfluss der politischen Verhältnisse in Ägypten auf den öffentlichen Sport konstatieren und deren Veränderungen mit der Zeit ganz ähnlich interpretieren. Dies sei im Folgenden nachgezeichnet.

Obwohl das Nationale Olympische Komitee schon sehr früh gegründet wurde (s.u.), begann erst Ende der 30er Jahre ein wachsendes gesellschaftliches Interesse am Sport. Dies ergab sich durch die Einführung von Sportangeboten in Schulen, Universitäten, der Gründung vieler Sportvereine und vieler Sportverbände. In dieser Zeit wurden Sportplätze in verschiedenen Gegenden des Landes geschaffen und mit angemessenen Sportgeräten und Sportmitteln ausgestattet. 
1934 wurde das Volkskomitee für Gymnastik gegründet, das für die Organisation der sportlichen Angelegenheit in Ägypten zuständig war. Außerdem wurden im Jahre 1937 mehrere sportliche Vereinigungen sowie Institutionen für die Ausbildung von Sportlehrern gegründet.

1951 organisierte Ägypten in Alexandria zum ersten Mal internationale Wettkämpfe für die Länder des Mittelmeeres. Aus Ägypten stammte auch die Idee der Organisation eines kontinentalen sportlichen Turniers für die Länder des afrikanischen Kontinents. Obwohl bereits 1929 in Alexandria beschlossen, wurde die Idee eines afrikanischen Turniers erst 1965 verwirklichte; Austragungsort dieses Turniers war Brazzaville, die Hauptstadt Kongos (jetzt Zaire).

Einen erneuten Schub erhielt das Sportsystem durch die Juli-Revolution 1952. Staatspräsident Gamal Abd el Nasser setzte eine neue Regierungspolitik um, die Veränderungen und Förderungen verschiedenster Bereiche als Folge der Unabhängigkeitserklärung und Reformvorhaben anstrebte. Einer der betroffenen Bereiche war der Sport (vgl. ÄGYPTISCHES MINISTERIUM FÜR JUGEND UND SPORT, 1999, 11f). Der Nasserismus übte auch einen großen Einfluss auf andere nordafrikanische Länder wie Tunesien, Algerien und Marokko aus. Es wurden Sportplätze gebaut und Sporthochschulen errichtet. Aufgrund der sozialistisch geprägten Politik ergab sich aber auch eine enge Anbindung bzw. ein reger fachlicher Austausch mit anderen sozialistischen Staaten wie der Sowjetunion. Der Sport hat in den Ländern, die diese Reformen durchgeführt haben, zur kulturellen Entwicklung beigetragen. Aus dieser Entwicklung ergab sich, dass in Ägypten viele neue Verwaltungsbeamte eingesetzt wurden, die für die verschiedenen Sportspiele und sportliche Aktivitäten verantwortlich waren. Es folgte eine Erneuerung der Regeln sowie in den Mitteln des Trainings und in der Sportverwaltung.

1955 wurde dann der Hohe Rat für die Betreuung der Jugendlichen und die Sporterziehung gegründet. Die ägyptischen Aktivitäten dehnten sich in der Folge regional, kontinental und international aus. 1957 forderte Ägypten zum ersten Mal die afrikanischen Länder auf, die afrikanische Meisterschaft im Fußball auszurichten. Das erste Turnier wurde im Februar 1957 in Khartum (Sudan) ausgerichtet. Das zweite Turnier fand 1959 in Kairo statt.

An den Daten wird ersichtlich, dass der Sport in Ägypten seit Beginn des zweiten Viertels des zwanzigsten Jahrhunderts bis in die sechziger Jahre eine erste Blüte erlebte, sodass die Kritiker des Sports diese Phase als die Goldene Epoche des 
ägyptischen Sports bezeichnen, weil sie durch große sportlichen Aufführungen gekennzeichnet war und Ägypten internationales Gehör fand (vgl. ÄGYPTISCHES MINISTERIUM FÜR JUGEND UND SPORT, 1997, 24f).

Der Sport wird bis heute in Ägypten (und den meisten arabischsprachigen Ländern) aber kaum professionell betrieben, sondern Sportler haben - mit Ausnahme erstklassiger Fußballer - lediglich Amateurstatus. Über die mit dem Sporttreiben verbundenen Wertvorstellung sich insgesamt feststellen: Sportlicher Erfolg gilt als erstrebenswert. Dies trifft insbesondere für internationale Wettkämpfe zu. In Ägypten haben die Sportarten Fußball und Handball sogar eine eigene Fankultur ausgebildet und das öffentliche Interesse ist hier auch für den nationalen Bereich sehr groß (z.B. während der Spielsaison Fernsehübertragungen etwa 2-3 Mal pro Woche).

Demgegenüber wird der Frauensport allerdings immer noch kritisch beäugt. Der kulturelle Hintergrund dafür - der Islam - ist aber nicht im eigentlichen Sinne sportfeindlich, sofern die islamischen Werte bei der Ausübung nicht verletzt werden (z.B. die Verhüllung der Frauen bei der Anwesenheit von Männern). Die Eingliederung von Frauen in den Sport ist bis heute noch nicht abgeschlossen und begann anfangs sehr zögerlich. 1936 wurde aber der erste Schritt getan, als die ersten zwei muslimischen Frauen offiziell an den Olympischen Spielen teilnahmen.

Obwohl der Leistungssport nun insgesamt von der Politik gefördert wird, wird dem allgemeinen Sporttreiben i.S. von Breiten- oder Freizeitsport von der Mehrheit der ägyptischen Bevölkerung immer noch eine geringe Bedeutung beigemessen. $\mathrm{Er}$ wird meist als Luxus betrachtet und ist kein fester Bestandteil der ägyptischen Freizeitkultur. Auch der Vereinssport genießt in Ägypten, das im Sport eine Vorreiterrolle unter den arabischen Ländern einnimmt, lediglich eine marginale Bedeutung. Dies ist aber nicht nur auf die Sichtweise von Sport zurückzuführen (einseitige Leistungsorientierung), sondern auch, weil die Mitgliedschaft in Sportvereinen vergleichsweise viel Geld kostet: Die Aufnahmegebühr beträgt mindestens 80 ,- $€$, was etwa vier durchschnittlichen Monatsgehältern entspricht; hinzu tritt ein jährlicher Mitgliedsbeitrag von etwa einem halben Monatsgehalt. Weiterhin existieren nur wenige und vielfach schlecht ausgestattete Sportstätten. Der Vereinssport hat also keinen festen Platz im Leben der Bevölkerung, da der Übergang von den 
vormals traditionellen Sportarten wie Bogenschießen, Speerspiele, Ringen und Reiten zu dem in Vereinen organisiertem Sport sich bisher noch nicht vollzogen hat (vgl. EL CHOULI, 1996a, 182f). Er wird - wenn überhaupt - von den gebildeten, wohlhabenden Schichten in den Städten ausgeübt, während er in den ländlichen Gebieten kaum existiert (vgl. EL CHOULI, 1996b, 178f). Im Folgenden sei das außerschulische Sportsystem näher anhand seiner Struktur betrachtet.

\subsubsection{Gegenwärtige Struktur}

Der Sport in Ägypten ist durch eine bestimmte Struktur zu beschreiben. An oberster Stelle steht das Ministerium für Jugend und Sport mit seinen Abteilungen. Dem ist das Nationale Olympische Komitee strukturell unterzuordnen, das sich wieder in die staatlich finanzierten Sportverbände mit innen untergeordneten Vereinen zerlegen lässt. Schließlich gibt es noch wenige private Sportanbieter.

Das Ministerium für Jugend und Sport wacht gewissermaßen über den Sport und erarbeitet Vorschläge für die allgemeine Politik. Es entwickelt Pläne und Programme für die Bevölkerung, insbesondere aber für die Jugend. Dabei nimmt es drei Verantwortlichkeiten wahr:

1. Die materielle und die fachliche Aufsicht, sowie die Aufsicht über die Durchsetzung der Pläne und der Programme der sportlichen Körperschaften, die sich administrativ dem Apparat unterwerfen, und die jährlich materielle Unterstützung für die Durchsetzung ihrer Pläne und Programme erhalten.

2. Das Anbieten von Dienstleistungen und die Durchführung nationaler Projekte wie der Wettbewerbe und der Sportfeste, der sportlichen Vorführungen und der Schulen.

3. Bau von sportlichen Einrichtungen in Zusammenarbeit mit der Ausstattungsund Einrichtungseinheit im Ministerium für Jugend und Sport.

In vielen Ländern der Welt, sowohl fortschrittlicher als auch unterentwickelter, interessieren sich die Regierungen nun für die Sportorganisationen, da sie sowohl national als auch international von großer Bedeutung im politischen, gesellschaftlichen und im wirtschaftlichen Bereich sind. Nachdem der Rat für Jugend und Sport 
seinen Namen in ,Ministerium für Jugend und Sport' geändert hat, ist es die administrative Dachorganisation, unter der sämtliche Organisationen wie ägyptisches Olympisches Komitee, Sportverbände und -vereine arbeiten.

\subsubsection{Nationales Olympisches Komitee}

Ägypten war eines der ersten Länder, das zur Wiederbelebung der Olympischen Aktivität beitrug, indem es an den modernen Olympischen Spielen 1896 teilnahm. Nach ihrer Gründung im Jahre 1910 trat das Ägyptische Olympische Komitee dem Internationalen Olympischen Komitee bei. Das Nationale Olympische Komitee ernannte in diesem Jahr seinen ägyptischen Vertreter. Mit diesem Beitritt war Ägypten der vierzehnte Staat, der dem Internationalen Olympischen Komitee beitrat.

1912 entschied sich das Ägyptische Olympische Komitee für die Teilnahme an den fünften Olympischen Spielen in Stockholm. An diesem Turnier nahm Ägypten mit einem Athleten an einem Schießwettbewerb teil. Nach dem Entwurf der Olympischen Flagge und ihrer Bestätigung durch das Olympische Komitee wurde sie zum ersten Mal auf dem Shataby Sportplatz in Alexandria anlässlich der zwanzigsten Erinnerungsfeier Ägyptens (1914) für die Olympischen Spiele aufgestellt (vgl. ÄGYPTISCHES OLYMPISCHES KOMITEE, 2004). Diese Teilnahme symbolisierte die Absicht, zukünftig regelmäßig an olympischen Wettkämpfen teilzunehmen. Danach begann Ägypten mit der Vorbereitung für die Teilnahme an den sechsten Olympischen Spielen in Berlin 1916, die aber wegen des ersten Weltkrieges ausfielen. 1920 nahm Ägypten dann jedoch an den siebten Olympischen Spielen in Antwerpen in Belgien teil und nimmt bis heute regelmäßig an den Olympischen Spielen teil. Insofern kommt dem Komitee eine hohe Bedeutung zu. 


\subsubsection{Sportverbände}

Zu Beginn des 20. Jahrhunderts (1907) fing man an, Verbände für Rudern, Radsport, Leichtathletik, Schwimmen und Fußball zu gründen. Die ägyptischen Sportverbände sind Körperschaften öffentlichen Rechts und haben ihren Sitz in Kairo. Sie haben relativ unabhängigen Status und operieren mit dem Ziel, ihre Sportart zu verbreiten, deren Niveau anzuheben und die Aktivität zwischen den Mitgliedern zu organisieren und zu fördern (vgl. MUSTAFA, 1998, 130f). Ein Dachverband für die Sportverbände - wie in Deutschland der DSB - existiert nicht. Daneben gibt es noch weitere, nicht-olympische Sportverbände, die aber in der öffentlichen Wahrnehmung keine Rolle spielen. Genauer bestehen 42 Verbände (24 Fachverbände olympischer Sportarten und weiterhin 18 nichtolympische Sportverbände). Die Verbände tragen und fördern die Sportbewegung in Ägypten (vgl. DER HOHE RAT FÜR JUGEND UND SPORT, 1997, 325f). Umseitige Tabelle (1) zeigt die Gründungsjahre der ägyptischen Sportverbände, die dem Nationalen Olympischen Komitee angeschlossen sind.

Das Gesetz über die Sportorganisationen in Ägypten bestimmt weiterhin, dass „die gemeinnützigen Sportorganisationen verantwortlich für die nationale sportliche wie finanzielle Leitung der jeweiligen Sportart sind. Dies schließt Verwaltung und Maßnahmen zur Steigerung des Leistungsniveaus ein" (vgl. DER HOHE RAT FÜR JUGEND UND SPORT, 1997, 64f). Die 24 Sportverbände bilden dann den Vorstand des Olympischen Komitees von Ägypten. Die Verbände haben Unterabteilungen in den Regionen, entsprechend dem Vorkommen der Sportart. Für die Bildung solch eines Regionalverbandes bedarf es mindestens sieben Vereine einer Sportart. Bei einer geringeren Zahl von Vereinen kann das Ministerium für Jugend und Sport die Bildung eines Regionalverbandes erlauben oder es weist die Vereine einem anderen, nahe liegenden Regionalverband zu. 


\begin{tabular}{|l|c|}
\hline Sportverband & Gründungsjahr \\
\hline Rudern & 1907 \\
\hline Radsport & 1910 \\
\hline Leichtathletik & 1910 \\
\hline Schwimmen & 1910 \\
\hline Fußball & 1921 \\
\hline Tennis & 1923 \\
\hline Fechten & 1929 \\
\hline Basketball & 1930 \\
\hline Gewichtheben & 1930 \\
\hline Boxen & 1930 \\
\hline Tischtennis & 1932 \\
\hline Ringen & 1934 \\
\hline Turnen & 1936 \\
\hline Reiten & 1942 \\
\hline Hockey & 1942 \\
\hline Volleyball & 1946 \\
\hline Schiessen & 1950 \\
\hline Bogenschiessen & 1950 \\
\hline Segeln & 1953 \\
\hline Handball & 1957 \\
\hline Judo & 1962 \\
\hline Fünfkampf und Biathlon & 1975 \\
\hline Taekwando & 1978 \\
\hline Badminton & 1991 \\
\hline
\end{tabular}

Tabelle 1: Gründungsjahre ägyptischer Sportverbände, die dem Olympischen Komitee angeschlossen sind (nach: ABD EL HAMEED \& EL CHOULI 2000).

\subsubsection{Sportvereine}

Die den Fachverbänden zugeordneten Sportvereine sind ebenso Körperschaften öffentlichen Rechts mit unabhängigem Status. Sie sind nicht an Gewinn orientiert, erfüllen allgemeinen Nutzen und bestehen aus nicht weniger als fünfzig Mitgliedern (vgl. ÄGYPTISCHES MINISTERIUM FÜR JUGEND UND SPORT, 2000).

Ziel des einzelnen Sportvereins ist die Förderung der Persönlichkeit, die sich an sozialen, gesundheitlichen, religiösen, psychischen, geistigen und an den regenerativen Funktionen des Sports orientiert. Dabei sollten vor allem bei der Jugend soziale Aspekte der Sporterziehung, wie die Ausbildung eines „vaterländischen Geistes" und des Zusammenhaltes zwischen den Mitgliedern wie auch die Förderung ihrer verschiedenen Begabungen als Ziele angestrebt werden. Damit sollten die notwendigen Mittel für die Gestaltung ihrer Freizeit gemäß den allgemeinen 
politischen Richtlinien und der Planung des Ministeriums der Jugend gewährleistet werden (vgl. ÄGYPTISCHES MINISTERIUM FÜR JUGEND UND SPORT, 1997, 21f).

Folgende Tabelle fasst nun die nationalen Sportverbände (im Rahmen des Olympischen Komitees) geordnet nach der Zahl ihrer Vereine zusammen. Daneben wird die Anzahl der Regionalverbände und die Anzahl der Mitglieder aufgelistet.

\begin{tabular}{|l|c|c|c|}
\hline Sportverband & $\begin{array}{c}\text { Anzahl der } \\
\text { Sportvereine }\end{array}$ & $\begin{array}{c}\text { Anzahl regionaler } \\
\text { Verbände }\end{array}$ & Mitglieder \\
\hline Fußball & 610 & 23 & 382644 \\
\hline Tischtennis & 482 & 24 & 135058 \\
\hline Basketball & 285 & 18 & 55404 \\
\hline Volleyball & 281 & 22 & 146375 \\
\hline Gewichtheben & 214 & 25 & 34540 \\
\hline Leichtathletik & 189 & 21 & 76909 \\
\hline Boxen & 166 & 12 & 21303 \\
\hline Handball & 148 & 21 & 59706 \\
\hline Ringen & 148 & 23 & 10899 \\
\hline Schwimmen & 134 & 7 & 17371 \\
\hline Judo & 132 & 10 & 23232 \\
\hline Hockey & 73 & 7 & 39241 \\
\hline Tennis & 73 & 9 & 15946 \\
\hline Taekwando & 61 & 10 & 18458 \\
\hline Turnen & 45 & 8 & 23666 \\
\hline Fechten & 34 & 2 & 8554 \\
\hline Rudern & 32 & 3 & 12290 \\
\hline Radsport & 31 & 14 & 25352 \\
\hline Schiessen & 29 & 3 & 13719 \\
\hline Badminton & 25 & 6 & $?$ \\
\hline Segeln & 19 & 3 & 15034 \\
\hline Reiten & 10 & 2 & 3211 \\
\hline Bogenschiessen & 8 & 1 & 14569 \\
\hline Moderner Fünfkampf & 8 & 2 & 10347 \\
\hline \multicolumn{1}{|c|}{ Summe } & $\mathbf{3 2 3 7}$ & $\mathbf{2 7 6}$ & $\mathbf{1 1 6 3 8 2 8}$ \\
\hline & & & \\
\hline Tahallo & & 7 & 0 \\
\hline
\end{tabular}

Tabelle 2: Sportverbände, Sportvereine und Mitglieder in Ägypten (Quelle: ÄGYPTISCHES MINISTERIUM FÜR JUGEND UND SPORT, 2001). 


\subsubsection{Talentförderung}

Am 01.04.1993 legte eine Abteilung des Ministeriums für Jugend und Sport („Sektion Meisterschaft“) eine Konzeption für die Talentförderung in Ägypten vor. Die Durchführung wird seither auch durch diese Sektion betreut (vgl. EL GAMAL, 1999, 149f). Das Ministerium für Jugend und Sport verfolgt mit dieser Konzeption folgende Ziele: Sportliche Talente sollen früh erkannt und gefördert werden. Hierzu sollen Mess- und Testverfahren verwendet werden, die speziell auf die Sportarten ausgerichtet sind. Die so gefundenen Talente sollen mit Trainingsprogrammen umfassend ausgebildet werden, um sie auf Zentren zur Meisterschaftsschulung vorzubereiten. Auf dieser Basis sollen die besten Sportlerinnen und Sportler für die diversen Nationalmannschaften vorbereitet werden (vgl. ÄGYPTISCHES MINISTERIUM FÜR JUGEND UND SPORT, 1997, 65f).

Um diese Ziele erreichen zu können, wurden entsprechende Zentren gegründet. Da die Talentförderung auf sportwissenschaftlicher Basis aufgebaut sein soll, wurden die Zentren an Orten etabliert, wo sportwissenschaftliche Institute oder Fakultäten vorhanden sind, um eine kompetente Betreuung zu gewährleisten. Solche Zentren befinden sich mittlerweile in verschiedenen Orten. Tabelle 3 gibt die einzelnen Orte mit den jeweils geförderten Sportarten sowie die Anzahl der geförderten Mannschaften bzw. Athleten wieder; sofern keine Unterteilung in Jungen (J) und Mädchen (M) vorgenommen wurde, stehen die Werte für die Förderung männlicher Athleten.

Ersichtlich wird an der Tabelle, dass die Förderung der männlichen Athleten (mit Ausnahme von Fechten und Turnen) deutlich überwiegt. Dies lässt zwei Vermutungen zu: Zum Einen könnte es sein, dass zu wenige junge Frauen Sport treiben und zum Anderen könnte angenommen werden, dass die in Kapitel 2.3.1 beschriebene Zurückhaltung gegenüber dem Frauensport sich auch auf administrativer Ebene manifestiert. Anhand von Alltagsbeobachtungen wird hier jedoch angenommen, dass beide zutreffen. 


\begin{tabular}{|c|c|c|c|c|c|c|c|c|c|c|c|c|c|}
\hline \multirow{2}{*}{$\begin{array}{r}\text { Sport } \\
\text { Stadt }\end{array}$} & \multirow[t]{2}{*}{ Fußball } & \multicolumn{2}{|c|}{ Basketball } & \multicolumn{2}{|c|}{ Volleyball } & \multirow[t]{2}{*}{ Hockey } & \multirow[t]{2}{*}{ Ringen } & \multirow[t]{2}{*}{ Boxen } & \multicolumn{2}{|c|}{ Fechten } & \multirow{2}{*}{$\begin{array}{l}\text { Leicht- } \\
\text { athletik }\end{array}$} & \multicolumn{2}{|c|}{ Turnen } \\
\hline & & $\mathbf{J}$ & M & $\mathbf{J}$ & M & & & & $\mathbf{J}$ & M & & $\mathbf{J}$ & M \\
\hline Alexandria & - & 1 & & 1 & - & - & 1 & - & 1 & 1 & - & - & - \\
\hline Assiut & 1 & 1 & - & - & - & - & - & - & - & - & 1 & - & - \\
\hline Aswan & - & - & - & - & - & - & - & - & - & - & 1 & - & - \\
\hline Bani Suif & - & - & - & 1 & - & - & - & - & - & - & 1 & - & - \\
\hline $\begin{array}{l}\text { El Bahr el } \\
\text { Amar }\end{array}$ & - & - & - & 1 & - & - & - & - & - & - & 1 & - & - \\
\hline El Buhaira & - & 1 & - & 1 & - & - & - & - & - & - & 1 & - & - \\
\hline El Faijum & - & - & - & 1 & - & - & - & - & - & - & 1 & - & - \\
\hline El Gedid & - & - & - & 1 & - & - & - & - & - & - & 1 & - & - \\
\hline $\begin{array}{l}\text { El Gharbi- } \\
\text { ya }\end{array}$ & 1 & 1 & 1 & - & - & - & - & - & 1 & 1 & 1 & - & 2 \\
\hline El Kalubia & - & 1 & - & - & - & - & 1 & - & - & - & 1 & - & - \\
\hline $\begin{array}{l}\text { El Man- } \\
\text { soura }\end{array}$ & - & - & - & 1 & - & - & - & - & - & - & 1 & - & - \\
\hline El Minia & 1 & - & - & 1 & - & - & - & - & - & - & 1 & - & - \\
\hline El Monofia & - & - & - & 1 & - & - & - & - & - & - & - & - & - \\
\hline El Sharqia & - & 1 & - & - & - & 1 & 1 & 1 & 1 & 1 & - & 1 & - \\
\hline El Wadi & - & - & - & 1 & - & - & - & - & - & - & 1 & - & - \\
\hline Dumiat & - & 1 & - & 1 & - & - & - & - & - & - & 2 & - & - \\
\hline Gizeh & 1 & - & 1 & & 1 & 1 & 1 & 1 & & & - & 1 & 1 \\
\hline Ismailja & - & 1 & - & - & - & - & - & - & - & - & 1 & - & - \\
\hline $\begin{array}{l}\text { Kafr el } \\
\text { Scheich }\end{array}$ & - & 1 & - & - & - & - & - & - & - & - & 1 & - & - \\
\hline Kairo & 2 & - & & 2 & 2 & - & 2 & 2 & 1 & 1 & 2 & - & 2 \\
\hline Kena & - & - & - & 1 & - & - & - & - & - & - & 1 & - & - \\
\hline Luxor & - & 1 & - & - & - & - & - & - & - & - & 2 & - & - \\
\hline $\begin{array}{l}\text { Marsa } \\
\text { matruh }\end{array}$ & - & - & - & 1 & - & - & - & - & - & - & 1 & - & - \\
\hline Port- Said & - & - & - & - & - & - & 1 & - & - & - & 2 & - & - \\
\hline $\begin{array}{l}\text { Schamal } \\
\text { Sinai, }\end{array}$ & - & 1 & - & 1 & - & - & - & - & - & - & 1 & - & - \\
\hline Suez & - & 1 & - & - & - & 1 & - & - & - & - & - & - & - \\
\hline Suhag & - & 1 & - & - & - & - & - & - & - & - & 2 & - & - \\
\hline
\end{tabular}

Tabelle 3: Anzahl der geförderten Athleten/Mannschaften in verschiedenen Stützpunkten, geordnet nach Sportarten (Quelle: STATISTISCHES ZENTRUM DES MINISTERIUMS FÜR JUGEND UND SPORT, 2001/2002).

Die Auswirkung, die vom Aufschwung des ägyptischen Sports ausging, erreichte nun auch die Bildungsinstitution Schule, die im folgenden Kapitel aufgegriffen wird. Nach langer Vernachlässigung erlangte der Sportunterricht in den Schulen größere Aufmerksamkeit, indem das „Technische Komitee der allgemeinen Verwaltung für die Betreuung von Sport und Jugend“ im Unterrichtsministerium 1964 je vereinheitlichte Lehrpläne für den Sportunterricht der Grund-, Mittel- und Oberschulen in Kraft setzte. Das Komitee zielte mit den neuen Lehrplänen darauf ab, die Schüler in Sport, Gesundheits- und Gesellschaftskenntnis zu erziehen sowie ihre motorischen Fähigkeiten und Fertigkeiten auszubilden (vgl. ABD EL MAQSUD, 1996, 78f). Seitdem nimmt der Sportunterricht in der Schule einen wichtigen Platz neben 
den anderen obligatorischen Unterrichtsfächern ein. Die Ansicht, Sport nur als Ergänzungsfach zu sehen, änderte sich, indem Sport ein Fach mit expliziten Zielen wurde, das eine wichtige Rolle innerhalb des Schulsystems einnahm und bis heute einnimmt.

\subsection{Schulsystem unter besonderer Berücksichtigung des Sport- unterrichts}

In Ägypten besteht Schulpflicht für Kinder ab sechs Jahren. Die Schul- und Universitätsausbildung ist kostenlos. Allerdings müssen viele Kinder ihre Schulausbildung aufgrund der wirtschaftlichen Situation ihrer Eltern abbrechen und die Schulpflicht wird nicht strikt befolgt.

\subsubsection{Historischer Abriss}

Als verständliches Ergebnis der Kolonialzeit war Ägypten für längere Zeit stark von Britischen Interessen beeinflusst ${ }^{8}$. Die Interessen hinsichtlich des Bildungssystems waren besonders darauf gerichtet, die Kader auszubilden, die dann die Bedürfnisse der Kolonialisten befriedigen konnten. Dies resultierte letztlich darin, dass die Ausbildung eines flächendeckenden Systems vernachlässigt bzw. eher verhindert wurde und dass das Analphabetentum in Ägypten während der Kolonialzeit 92\% bei den Männern und 99\% bei den Frauen erreichte (vgl. BADRAN, 1995, 191f). Die weiteren Auswirkungen der Kolonialzeit wie Verhinderung der Industrialisierung, Landflucht, Arbeitslosigkeit und Armut einerseits sowie ausschweifender Luxus am Königshof Faruks und Korruption andererseits resultierten letztlich in der Revolution im Jahre 1952 durch General Nagib, der 1953 erster Staatspräsident der Republik wurde. Nach dessen Absetzung durch Gamal Abd el Nasser mit seinen „freien Offizieren“ 1954 wurde die Revolution fortgeführt und intensiviert. Wie

\footnotetext{
${ }^{8}$ Vom Ende des 19. Jahrhunderts bis 1922 war Ägypten durch die Engländer besetzt. Als Kolonialmacht waren sie jedoch auch nach der offiziellen Unabhängigkeitserklärung und Umwandlung in ein Königreich 1922 bis 1952 im Land.
} 
schon angeklungen entstand damit ein neues politisches und gesellschaftliches Klima, bei dem es der neuen Staatsführung zunächst darauf ankam, den Ägyptern eine neues Gefühl der Würde und ein neues Selbstbewusstsein zu geben und Maßnahmen zur Durchsetzung sozialer Gerechtigkeit und staatlichen Unabhängigkeit einzuleiten. Dies war auch der Startpunkt für die Entstehung eines flächendeckenden Bildungssystems in Ägypten. Mit dem neu formulierten „Recht auf Bildung“ war aber gleichzeitig auch der entwicklungspolitische Gedanke verbunden, Qualifikationen für die längerfristige Förderung des Staates herauszubilden. Der manifeste Beginn war die Herausgabe des Ausbildungsgesetzes von 1953, das den grundlegenden Ideen der Revolution Rechnung trug wie insbesondere gleichwertigen Ausbildungschancen. Das Gesetz legte einerseits das Recht auf kostenlose Ausbildung fest, womit die Beschränkung des Zugangs zur Bildung durch die gehobenen und reichen Schicht der Gesellschaft aufgehoben wurde, und andererseits die Einführung der Schulpflicht. Dieses Gesetz änderte weiterhin auch die Dauer der Ausbildung in den verschiedenen Phasen, nutzte aber dabei schon bestehende Strukturen (vgl. ABD EL HAMID, 1998, 49f).

In der Grundschule wurde die Ausbildungszeit von neun Jahren vor der Revolution nun auf sechs Jahre beschränkt. In den weiterführenden Mittelschule hatte sich die Ausbildungszeit dagegen von zwei Jahren auf vier Jahren gesteigert. Die Ausbildungszeit an den höheren Schulen blieb bei drei Jahren. Man bereitete sich mit dieser Veränderung auf die Zunahme der Schülerpopulation vor, denn nach der Revolution erhöhte sich der Anteil der Schüler unter den sechs- bis zwölfjährigen Kindern Ägyptens von 33\% auf 87\%. Daneben wurde eine neue Schulart, nämlich die Gewerbeschulen festgeschrieben und damit eine weitergehende Qualifikation durch entweder allgemeine oder berufliche Oberschulen (sog. Institute) gesichert (vgl. RUSTOM, 1994, 53f). Die allgemeinen Oberschulen bereiten die Schüler auf das Studium an den Universitäten vor (s.u.), wohingegen die Gewerbeschulen auf die Arbeit in den Firmen vorbereiten soll, um so den Bedarf des Arbeitsmarktes nach ausgebildeten Arbeitern im industriellen Bereich zu decken, womit auch die Umwandlung der ägyptischen Gesellschaft von einer landwirtschaftlichen zu einer landwirtschaftlich-industriellen Gesellschaft ermöglicht werden sollte (der Anteil der industriellen Produktion, der vor 1953 lediglich 6,7\% des gesamten nationalen Einkommens betrug, steigerte sich bis zum Jahr 1956 auf 26,7\%; vgl. HASSAN, 1996, 144f). Mit diesen Veränderungen kamen dann vielfache praktische Maß- 
nahmen in Gang (beispielsweise stammen fast alle derzeitigen Schulgebäude aus dieser Zeit).

Im akademischen Bereich hatte der Staat neue Universitäten eingerichtet, um die zunehmende Zahl der Schüler, die die Oberschule hinter sich hatten, aufnehmen zu können. Universitäten wurden in Alexandria, Zagazig und El Mansoura gegründet, nachdem das akademische Studium sich bis dahin im Wesentlichen auf eine einzige Universität, die sogenannte Farouk Universität (heute: Kairo Universität) beschränkt hatte. Die Veränderung in Ägypten betraf aber auch die Ausbildung in den religiösen Anstalten. So spielte die berühmte Azhar Institution eine wesentliche Rolle in der Entwicklung der neuen ägyptischen Gesellschaft. Die Azhar Universität führte im Jahre 1930 Fakultäten für Naturwissenschaften und Sozialwissenschaften neben den traditionellen religiösen Wissenschaften ein, was sie zu einem Studienplatz für viele Studenten aus den verschiedenen islamischen Ländern machte und bis heute noch macht. BADRAN, EL BUHI \& MOHAMMED (2001, 81f) berichten von mehr als 90.000 Studenten aus verschiedensten islamischen Ländern. Die ehrwürdige Azhar Institution hat weiterhin ein hohes Ansehen und Einfluss in vielen Ländern der islamischen Welt, besonders die theologische Fakultät, weil sie eine gemäßigte, die verschiedenen Richtungen überbrückende Einstellung hinsichtlich religiöser Fragen hat. Deshalb unterstützte der Staat (und unterstützt noch heute) die Bemühungen der Azhar, um so die gemäßigten religiösen Ausbildung in der ägyptischen und islamischen Gesellschaft zu verbreiten.

1968 wurden wichtige Modifikationen zum Ausbildungsgesetz von 1953 verabschiedet, sodass das Unterrichtsfach der islamischen religiösen Erziehung für die muslimischen Schüler und das Unterrichtsfach der christlich-religiösen Erziehung für die christlichen Schüler innerhalb des schulischen Stundenplanes eingesetzt wurde. Mit Blick auf die Bildungsförderung wurde 1982 die Schullandschaft nochmals entscheidend verändert. Unter dem Schlagwort „Bildung ist nötig" wurde die Schulpflicht von der Grundschule zusätzlich auf die Mittelschule ausgedehnt. Damit wurde eine „Basisbildung" zum Kern des ägyptischen Schulsystems (vgl. RAYAAN, 1992, 87f).

Nach 1982 begann der Staat (wenn auch etwas zögerlich), das Bildungssystem der Revolution zu überprüfen, um die Ausbildungsqualität weiter verbessern zu können. Dabei stellte sich insbesondere heraus, dass der Mangel an Finanzierungsmitteln und Ausrüstungsgegenständen auf Kosten der Qualität ging. Zwar 
ging die Rate der Analphabeten zwischen 1980 und 1997 deutlich zurück, liegt aber bei der Bevölkerung über 15 Jahre immer noch bei $58 \%$ weiblichen bzw. 35\% männlichen Einwohnern (vgl. ZENTRALES AMT FÜR ÖFFENTLICHE ERHEBUNGEN UND STATISTIK, 2001; CIA, 2004). Dies führte zwar einerseits zu der Einsicht, dass das System verbessert werden müsst. Andererseits suchte man eine Lösung in einer Intensivierung und Konzentration auf sprachliche und technisch orientierte Unterrichtsfächer, während andere stärker eingeschränkt wurden, zu denen auch der Sportunterricht gehörte (vgl. EL CHOULI, 1998, 37f). Aufgrund der finanziellen Lage des Staates stammen die Sportstätten immer noch aus der Zeit Nassers und werden kaum verbessert; aufgrund des warmen Klimas gibt es auch nur sehr selten eine Sporthalle und so findet der Sportunterricht im Freien statt. Besonderes (auch finanzielles) Augenmerk gilt heute sog. „zukunftsträchtigen“ Lehrinhalten wie der Umgang mit neuen Technologien und Medien. Daneben soll aber auch eine Charakterentwicklung keinesfalls ausgeblendet, sondern als gleichrangig herausgestellt werden (vgl. BADRAN, 1995, 191f).

Wie schon erwähnt, wurde das ägyptische Schulsystem durch die Zeit der englischen Besetzung beeinflusst und so spiegelte sich dies auch in der Entwicklung des Sportunterrichts wider. Obwohl der Sportunterricht 1953 als Fach mit übernommen wurde, konnte der Einfluss des britischen Systems erst 1978 durch die ägyptische Administration beseitigt werden.

Das „Britische System“ sah den Sportunterricht als Hauptfach vor. So war es auch nicht verwunderlich, dass sich nach der Umwandlung in ein Königreich 1922 das damalige Kultusministerium 1925 für die Einführung des Sportunterrichts als Hauptfach entschied. Die Lehrer dieses Faches waren die Absolventen der englischen Militärschulen und der Sportunterricht beschränkte sich in dieser Zeit auf einige wenige gymnastische Übungen. Die 1939 gegründete und dem Ministerium für soziale Angelegenheiten zugeordnete Abteilung ,Sport' hatte dann die Aufgabe, den Sport zu beaufsichtigen und ihn in der Gesellschaft zu verbreiten. Diese Abteilung beschloss die zusätzliche Einführung des Turnens im Sportunterricht (vgl. EL CHOULI, 1998, 41f). Da dem Sport als Unterrichtsfach aber dennoch keine hervorgehobene Bedeutung zugeschrieben wurde und die Anstrengungen beim Aufbau des allgemeinen Schulsystems (und hier insbesondere der intellektuellen Entwicklungsförderungen) nach 1953 im Vordergrund standen, wurde erst 
1964 eine weitere und allgemein ausgerichtete Verwaltungsabteilung des Kultusministeriums zur Betreuung des Arbeitsbereichs ,Sport und Jugend' gegründet. Dieser Abteilung wurde die Verantwortung übertragen, den Lehrplan des Sportunterrichts für verschiedene Schulphasen zu entwerfen, da die Zuständigen langsam feststellten, dass der Sportunterricht wie die anderen Unterrichtsfächer notwendig zum Erreichen bestimmter Bildungsziele hilfreich war (vgl. ABD EL HAMID, 1998, 49f). Trotz der dadurch zum Ausdruck kommenden langsamen Lösung vom britischen System hatten bis 1978 mehr als 20.000 ägyptische Armeeoffiziere den Sportunterricht gelehrt, und zwar genauso wie die englischen Armeeoffiziere vor der Revolution dieses Fach lehrten.

Nach der Entstehung der ersten Sportinstitute (1968 in Alexandria und Kairo) - als dann verstärkte Zweifel an den angewandten Methoden aufkamen - konnten die wenigen Sportwissenschaftler die Zuständigen des ägyptischen Ministerium für Erziehung und Unterricht 1978 davon überzeugen, die Sportlehrerausbildung in den Verantwortungsbereich der Universitäten zu legen und eine qualifizierte pädagogische Ausbildung zu sichern (vgl. BADRAN, EL BUHI \& MOHAMMED, 2001).

1990 fanden die Bemühungen dann einen weiteren gesellschaftspolitischen Niederschlag, bei dem durch das Kultusministerium ein Rahmen für die Inhalte des Sportunterrichts festgeschrieben wurde. Dabei erstellten Experten den noch heute gültigen Lehrplan (vgl. ABD EL AZIEM, 1993; siehe weitergehend Kapitel 3).

\subsubsection{Gegenwärtige Struktur}

Das ägyptische Bildungssystem ist so aufgebaut, dass die Kinder im Alter von vier bis sechs Jahren einen Kindergarten besuchen können. Anschließend werden sie in die Grundschule eingeschult.

Die Grundschule umfasst eine Ausbildung von fünf Jahren und ist zweigeteilt. Im ersten Teil (drei Jahre) werden - neben religiösen Inhalten - Grundkenntnisse z.B. in Lesen, Schreiben oder Rechnen vermittelt. Im zweiten Teil (zwei Jahre) sollen die Kenntnisse und Fertigkeiten auf die Verrichtung der täglichen Aktivitäten ausgerichtet sein. Grundsätzlich liegt die Unterrichtszeit zwischen 8 und 13 Uhr. Die Unterrichtsfächer der Grundschule (Arabisch, Mathematik, Religion, Englisch, 
Praktische Landwirtschaft, Sozialverhalten und Sport) werden je mit zwei Unterrichtseinheiten (à 45 Min.) pro Woche durchgeführt; in der Regel gibt ein Lehrer an sechs Schultagen dabei nur ein Unterrichtsfach ${ }^{9}$. Dementsprechend unterrichten in der Grundschule mindestens acht Fachlehrer. Die wöchentliche Unterrichtszeit von 16 Einheiten bedeutet, dass in der Grundschule auch viele Freistunden bestehen. Der Abschluss der Grundschule ermöglicht dann den Zugang zu weiterführenden Schulen. Für diesen Abschluss muss eine staatlich vorgegebene, schriftliche Prüfung abgelegt werden und dabei ein Ergebnis von mindestens 50\% der möglichen Punktzahl erreicht werden. Wird die Prüfung nicht bestanden, muss die letzte Klasse wiederholt werden (vgl. KARIM, BADRAN \& MOHAMMED, 2000, 135f). Für Sport als Fach findet jedoch keine Prüfung statt und im Zeugnis taucht das Fach auch nicht auf.

Die dreijährige Mittelschule soll die Schüler für weiterführende Schulen vorbereiten und erziehen. Dabei soll innen die Möglichkeit gegeben werden, ihre Neigungen zu entdecken und zu entwickeln sowie die Richtung zu wählen, in der sie sich weiterbilden möchten (vgl. HASSAN, 1996, 144). Innerhalb der Mittelschule muss in jedem Unterrichtsfach (die gleichen Fächer wie in der Grundschule plus ein Fach „Wissenschaft") jährlich eine Prüfung bestanden werden. Wird die Prüfung in einem Fach nicht bestanden (d.h. in den schriftlichen Prüfungen weniger als $40 \%$ der möglichen Punktzahl erreicht), so muss die Klasse wiederholt werden. Da dies jedoch pro Klassenstufe nur ein Mal möglich ist, bedeutet ein zweites NichtBestehen das Ende der Schulzeit, was allerdings sehr selten vorkommt. Nach der dritten Klasse wird wieder eine zentrale staatliche Prüfung abgelegt, bei der pro Fach mindestens $40 \%$ und von allen Fächern insgesamt $50 \%$ der möglichen Punkte zum Bestehen erreicht werden müssen. Mit Bestehen der Mittelschule wird die "Mittlere Reife" erlangt und ermöglicht bei einem Prüfungsergebnis von mehr als $70 \%$ der möglichen Punktzahl den Besuch der Oberschule. Wird ein schlechteres Ergebnis erzielt, kann eine Gewerbeschule (Handelsschule, Berufsschule oder Landwirtschaftsschule) besucht werden. Auch für die Mittelschule ist wieder festzustellen, dass das Fach Sport nicht geprüft wird und keinen Eingang in die Zeugnisse findet. Schließlich liegt ein weiterer bedeutsamer Aspekt der Mittelschule

\footnotetext{
${ }^{9}$ Diese Struktur von zwei Unterrichtseinheiten pro Unterrichtsfach an sechs Tagen pro Woche und die täglichen Schulzeit (8-13 Uhr) gilt auch für die nachfolgenden Schulformen.
} 
darin, dass hier im Gegensatz zur Grundschule eine Trennung von Jungen und Mädchen vorgenommen wird. Während in der Grundschule Mädchen und Jungen gemeinsam unterrichtet werden (entweder von Lehrern oder Lehrerinnen), so gibt es ab der Mittelschule nur noch reine Jungen- oder Mädchenschulen, die von gleichgeschlechtlichen Lehrern unterrichtet werden. Dies gilt auch für die nachfolgenden Oberschulen.

In der Oberschule werden die humanistische sowie die technische Bildung drei Jahre lang vertieft und in verschiedene Richtungen erweitert. Daraus resultieren deutlich mehr Unterrichtsfächer: Arabisch, Mathematik, Religion, Englisch, Praktische Landwirtschaft, Sozialverhalten, Französisch, Biologie, Geografie, Geschichte, Physik, Statistik, Philosophie und Sport. Dabei müssen ebenso jährliche Prüfungen mit nahezu der Mittelschule identischen Kriterien bestanden werden (ebenfalls keine Sportnote/Prüfung). Weil auch hier nur ein Lehrer ein Unterrichtsfach gibt, stehen der Oberschule mindestens 15 Lehrer zur Verfügung. In der Gewerbeschule erfolgt eine gezielte Berufsausbildung (vgl. HASSAN, 1996, 146f).

Wird die Oberschule, in der es keine Freistunden mehr gibt, wiederum mit einem guten Prüfungsergebnis abgeschlossen, so ermöglicht dies den Besuch der Universität. Die einzelnen Fakultäten (z.B. für Sport, Medizin, Maschinenbau, Mathematik) haben dabei einen vom Ministerium für Erziehung und Unterricht unterschiedlich vorgegebenen Numerus clausus, der jährlich bestimmt wird ${ }^{10}$. Absolventen mit weniger Punkten haben die Möglichkeit, spezialisierte Institute (wie pädagogische Institute, soziale Institute, kaufmännische Institute, Gesundheitsinstitute oder andere Gewerbeinstitute) zu besuchen. Gleichzeitig ermöglicht aber auch ein Abschluss der Gewerbeschule mit einem höher als 70\%-igen Gesamtergebnis den Besuch eines Institutes.

Der Abschluss des Institutes lässt sozusagen über den zweiten Bildungsweg wiederum den Besuch der Universität zu. Hier ist neben dem Erreichen eines über 70\% liegenden Prüfungsergebnisses (vgl. ÄGYPTISCHES MINISTERIUM FÜR ERZIEHUNG UND UNTERRICHT, 1998, 24) im Sportfach jedoch zusätzlich noch eine Aufnahmeprüfung notwendig (siehe Kapitel 3.3). Umseitige Grafik veranschaulicht zusammenfassend die heutige Bildungsstruktur (Abbildung 2).

\footnotetext{
${ }^{10}$ Für Medizin liegt dieser Numerus clausus etwa bei $95 \%$, für Sport etwa bei $70 \%$.
} 


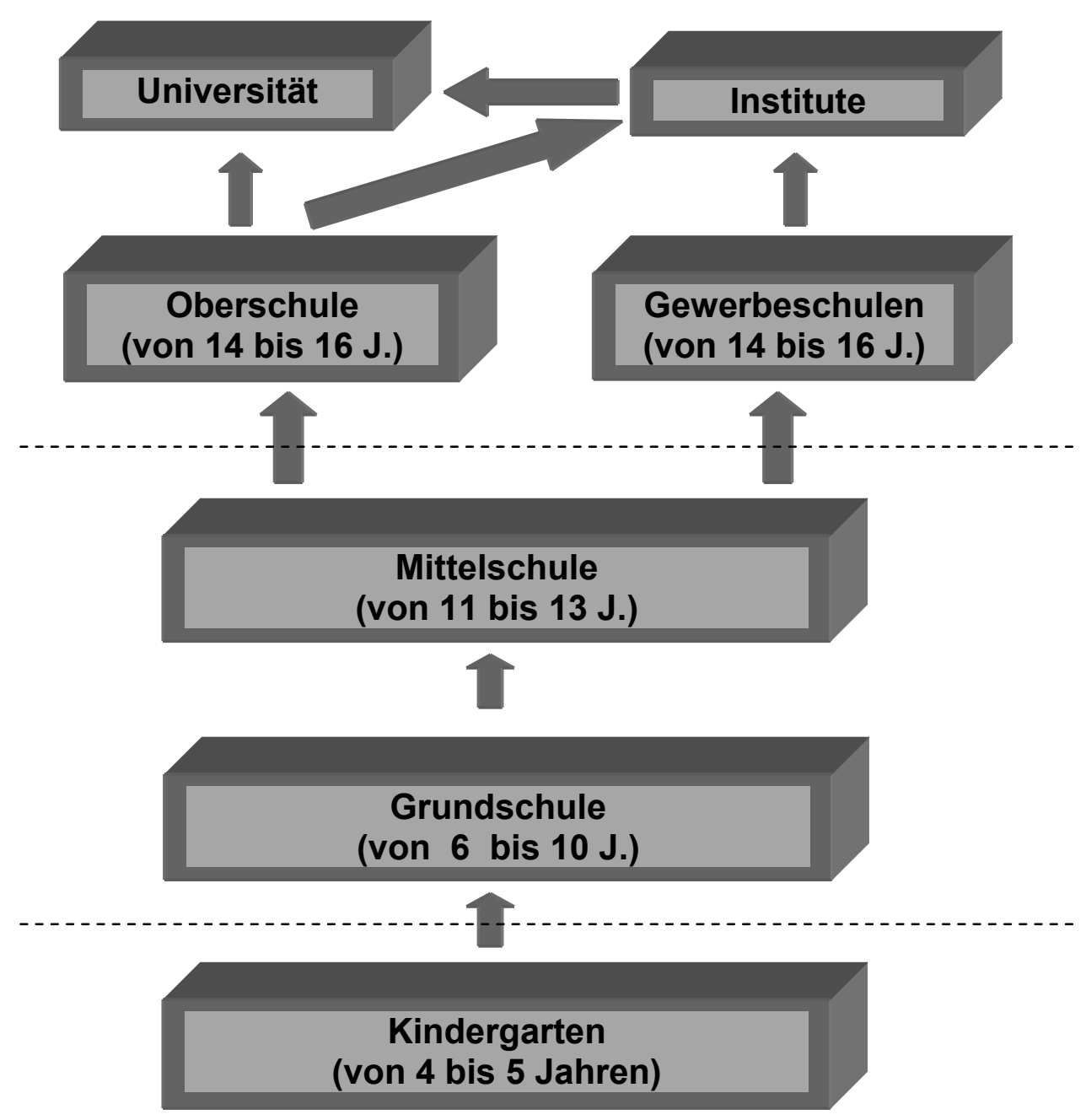

Abbildung 2: Überblick zum Ägyptischen Bildungssystem.

In Ägypten besuchen von den weiterführenden Schulen nach der Mittelschule insgesamt etwa $60 \%$ die Oberschule und $40 \%$ eine Gewerbeschule (Handelsschule, Berufsschule oder Landwirtschaftschule) (vgl. SALIH \& SHEHATA, 1994, 52). Von den Oberschülern setzen etwa $75 \%$ ihre Ausbildung in der Universität fort und lediglich $25 \%$ im Institut. Von den Gewerbeschulabsolventen setzen nur wenige (etwa 15\%) ihre Ausbildung in einem Institut fort und lediglich 10\% derjenigen, die das Institut abschließen, besuchen danach eine Universität.

Bedenkt man, dass 1995 ca. 38\% der Bevölkerung unter 15 Jahre alt war, wird deutlich, welche Anforderungen das Schulsystem zu bewältigen hat (vgl. BADRAN, EL BUHI \& MOHAMMED, 2001). In Ägypten steht weiterhin nicht genügend Schulraum zur Verfügung, um eine solch große Zahl von Schülerinnen und 
Schüler aufnehmen zu können. Die folgende Tabelle vermittelt einen Überblick zur aktuellen Schulsituation.

\begin{tabular}{|c|c|c|c|c|c|c|}
\hline & \multirow[t]{2}{*}{ Grundschule } & \multicolumn{2}{|c|}{ Mittelschule } & \multicolumn{2}{|c|}{ Oberschule } & \multirow[t]{2}{*}{ Gesamt } \\
\hline & & Jungen & Mädchen & Jungen & Mädchen & \\
\hline Schulen & 15608 & 4376 & 3168 & 936 & 684 & 24772 \\
\hline Klassen & 173529 & 58995 & 39329 & 14710 & 9808 & 296371 \\
\hline Schüler/innen & 7351126 & 2478553 & 1866803 & 623975 & 415983 & 12736440 \\
\hline Lehrer & 314561 & 100296 & 95043 & 38524 & 41186 & 589610 \\
\hline - männlich & 149959 & 88897 & 22224 & 36424 & 14519 & 312023 \\
\hline - weiblich & 164602 & 11399 & 72819 & 2100 & 26662 & 277582 \\
\hline \multirow{2}{*}{$\begin{array}{l}\text { Durch- } \\
\text { schnittliche } \\
\text { Anzahl von } \\
\text { Schülern/ } \\
\text { Schule }\end{array}$} & \multirow{2}{*}{470} & 566 & 589 & 667 & 608 & \multirow{2}{*}{467} \\
\hline & & \multicolumn{2}{|c|}{576} & \multicolumn{2}{|c|}{642} & \\
\hline \multirow{2}{*}{$\begin{array}{l}\text { Durch- } \\
\text { schnittliche } \\
\text { Anzahl von } \\
\text { Schülern/ } \\
\text { Klasse }\end{array}$} & \multirow[b]{2}{*}{42} & 42 & 47 & 42 & 42 & \multirow[b]{2}{*}{43} \\
\hline & & \multicolumn{2}{|c|}{44} & \multicolumn{2}{|c|}{42} & \\
\hline \multirow{2}{*}{$\begin{array}{l}\text { Durch- } \\
\text { schnittliche } \\
\text { Anzahl der } \\
\text { Lehrerl } \\
\text { Schule }\end{array}$} & \multirow{2}{*}{20} & 23 & 30 & 41 & 60 & \multirow{2}{*}{-} \\
\hline & & \multicolumn{2}{|c|}{26} & \multicolumn{2}{|c|}{49} & \\
\hline
\end{tabular}

Tabelle 4: Überblick zur aktuellen Schulsituation (Quelle der Daten, oberer Teil: STATISTISCHES ZENTRUM DES MINISTERIUMS FÜR ERZIEHUNG UND UNTERRICHT, 2001/2002).

Hieran wird deutlich, dass an den ägyptischen Schulen grundsätzlich sehr große Klassen unterrichtet werden und relativ viele Lehrerinnen und Lehrer zur Verfügung stehen: In den Grundschulen unterrichten etwa 20 Lehrer. Weil ein Fachlehrer lediglich ein Unterrichtsfach gibt und 8 Fächer unterrichtet werden, stehen einer Schule etwa zwei Fachlehrer pro Schule zur Verfügung. Diese müssen dann ca. 11 Klassen unterrichten. Bei zwei Unterrichtseinheiten pro Woche und der Sechs-Tage-Woche resultieren daraus etwa zwei Unterrichtseinheiten pro Tag. Dies bedeutet also eine geringe Arbeitszeit, aber eine relativ hohe Belastung während dieser Arbeitszeit. Größtes Problem ist entsprechend die Klassengröße. Praktisch identisch ist das Problem in den Mittel- und Oberschule. Der Mittelschule 
stehen durchschnittlich drei Fachlehrer für 13 Klassen zur Verfügung. Hieraus ergibt sich ein Verhältnis von neun Unterrichtseinheiten pro Lehrer und Woche. In der Oberschule werden 15 Unterrichtsfächer gelehrt. Bei gemittelten 49 Lehrern pro Schule resultiert auch hier eine tägliche Unterrichtszeit von zwei Einheiten. Dieses Verhältnis trifft allerdings nur bedingt auf den Sportunterricht zu, wie nachfolgende Tabelle verdeutlichen soll. In Bezug auf die Schulformen sei daran erinnert, dass in den weiterführenden Mädchenschulen nur Sportlehrerinnen und den weiterführenden Jungenschulen nur Sportlehrer unterrichten, sodass die relevanten Zahlen zum nachfolgenden Vergleich entsprechend hervorgehoben sind.

\begin{tabular}{|l|c|c|c|c|}
\hline & Grundschule & Mittelschule & Oberschule & Gesamt \\
\hline \hline Sportlehrer & 3931 & $\mathbf{4 6 4 8}$ & $\mathbf{1 5 5 3}$ & 10132 \\
\hline Sportlehrerinnen & 2403 & $\mathbf{2 9 8 6}$ & $\mathbf{9 0 4}$ & 6293 \\
\hline Gesamt & $\mathbf{6 3 3 4}$ & 7634 & 2457 & $\mathbf{1 6 4 2 5}$ \\
\hline
\end{tabular}

Tabelle 5: Anzahl der Sportlehrerinnen und Sportlehrer in Ägypten (Quelle: STATISTISCHES ZENTRUM DES MINISTERIUMS FÜR ERZIEHUNG UND UNTERRICHT, 2001/2002).

Setzt man die Zahlen mit denen aus Tabelle 4 ins Verhältnis, d.h. bezieht man die Anzahl der Sportlehrkräfte auf die Zahl der Grundschulen (15 608), so bedeutet dies, dass etwa 2 Sportlehrer pro Grundschule zur Verfügung stehen und diese dann durchschnittlich 470 Schüler betreuen. Dies trifft auch auf die Oberschulen zu. Hier kommt ebenfalls etwa eine Sportlehrkraft auf ca. 610 bzw. 670 Schüler(innen). In den Mittelschulen unterrichtet allerdings gerade mal ein Sportlehrer etwa 580 Schüler. Dementsprechend ist die Arbeitsbelastung der Sportlehrer in den Oberschulen leicht und den Mittelschulen deutlich erhöht: In der Oberschule muss ein Sportlehrer bzw. eine Sportlehrerin ca. 15 Einheiten (etwa 3 täglich) und in den Mittelschulen ca. 26 (etwa 4 täglich) unterrichten. Damit ist ein Mangel an Sportlehrkräften für die weiterführenden Schulen zu konstatieren.

Ein weiteres Merkmal des Schulsystems ist das nachmittägliche Freizeitangebot. Die einzelnen Fachlehrer bieten vielfach in ihrem Fachbereich Aktivitäten an, so auch der Sportlehrer den Schulsport. Dies ist zwar als traditionelle Aufgabe des Sportlehrers gedacht (vgl. ÄGYPTISCHES MINISTERIUM FÜR ERZIEHUNG UND UNTERRICHT, 1995; 2001), erfolgt aber freiwillig. Mit dem Schulsport wird 
das Ziel verfolgt, das Leistungsniveau in den einzelnen Sportarten zu verbessern und insbesondere talentierte Schüler zu fördern ${ }^{11}$. Die damit verbundene Leistungsorientierung zeigt sich an Schulwettkämpfen, aber auch weitergehend an studentischen Wettkämpfen (mit dem gleichen System). Damit soll eine Brücke zum außerschulischen Sport geschlagen werden, denn die besten der nationalen Wettkämpfe werden auch zu Kadertrainings der Nationalmannschaften eingeladen. Wettkämpfe von den Sporteinrichtungen werden rund um das Jahr angeboten. Sie finden auf Schulebene, zwischen den Schulen eines Distriktes oder auf Landesebene statt. Ähnliches trifft für die Universitäten zu. Die Wettkämpfe werden entweder vom Sportverband der Universitäten oder der Schulen ausgerichtet. Sport ist eine der wichtigsten Betätigungen zur Freizeitgestaltung. Jedoch wird aus einer Statistik des STATISTISCHEN ZENTRUMS DES MINISTERIUMS FÜR JUGEND UND SPORT (2001/2002) deutlich, dass lediglich in der Hälfte der Schule Freizeitaktivitäten anbieten. Umgekehrt betreiben gut zwei Drittel der Studenten Sport in der Freizeit. In den Schulen werden in folgenden Sportarten Wettkämpfe durchgeführt: Fußball, Basketball, Volleyball, Handball, Leichtathletik, Schwimmen, Tischtennis, Ringen und Judo. An diesen Wettkämpfen beteiligen sich etwa $12 \%$ der Schüler (vgl. ÄGYPTISCHES MINISTERIUM FÜR ERZIEHUNG UND UNTERRICHT, 1995, 95f). Die sportlichen Siege des jeweiligen Bildungsinstituts werden dann auch als Siege derer Leiter aufgefasst. Daher stehen Sportlehrer (bzw. „Trainer des Schulsports") bei Schülern und Studenten in hohem Ansehen (vgl. El HAMAHMI \& ABD EL AZIZ, 1998, 201f).

Die Bedeutung des Sportunterrichts und des Schulsports zur Anhebung des sportlichen Niveaus zeigt eine Untersuchung des Ministeriums für Jugend und Sport des Jahres 1998. Daraus ist zu Entnehmen, dass es zur weiteren Verbesserung einer noch größeren finanziellen Unterstützung bedarf, um Geräte, Ausrüstung, Lehrpersonal sowie Sportplätze und Programme bereitstellen zu können. Weiter fordert die Studie, die Schul- und Universitätsliga nicht nur für eine Auswahl, sondern wieder für alle Sportarten einzuführen (vgl. ÄGYPTISCHES MINISTERIUM FÜR JUGEND UND SPORT, 1999, 145f). Die Bedeutung des Schul- und Universitätssportes geht aber noch weiter, denn Schulen und Sportinstitute stellen auch

\footnotetext{
${ }^{11}$ Üblich ist ein sportartspezifisches Training einmal pro Woche. Das Angebot richtet sich nach den spezifischen Kenntnissen und Neigungen der Sportlehrer.
} 
die Sportanlagen, die von den Sportvereinen genutzt werden können. Die ägyptischen Universitäten bieten häufig gar Sportplätze, die den Richtlinien und Bestimmungen der internationalen Sportverbände entsprechen. So werden beispielsweise die Sportplätze der Universitäten von Ain Schams, Kairo und Helwan als Austragungsplätze der afrikanischen Spiele genutzt.

Abschließend sei noch auf die Lehrerausbildung und -administration eingegangen.

Die Ausbildung zum Grundschullehrer dauert fünf Jahre. Sie erfolgt an pädagogisch ausgerichteten Lehrerbildungsinstituten, die keinen Universitätsstatus besitzen. Eine Spezialisierung auf bestimmte Fächer erfolgt erst in den letzten beiden Jahren dieser Ausbildung. Voraussetzung für die Aufnahme an ein Lehrerbildungsinstitut ist ein Oberschulabschluss oder der Abschluss einer Gewerbeschule. Sport kann dabei als Nebenfach gewählt werden. Ein erfolgreicher Abschluss der Fortbildung berechtigt zum Unterrichten von Klasse eins bis Klasse acht, also auch ein Unterrichten in der Mittelschule.

Wer einen qualifizierten Grundschullehrerabschluss besitzt, kann seit 1995 nach einer Aufnahmeprüfung auch an einer Universität studieren. Dieses sechssemestrige Studium umfasst die Lehrbefähigung für Mittel- und Oberschule. Bei diesem Studium kann Sport dann auch als Hauptfach gewählt werden. Von dieser Fortbildungsmöglichkeit machen jedoch nur wenige Grundschullehrer gebrauch, da sie neben der Berufstätigkeit stattfindet.

Der übliche Weg zu einem achtsemestrigen Studium an einer Universität erfolgt jedoch über die Oberschule mit der direkten Legitimation zum Unterrichten in Mittel- und Oberschule (vgl. ÄGYPTISCHES MINISTERIUM FÜR ERZIEHUNG UND UNTERRICHT, 1998, 30). Die Prüfungen laufen studienbegleitend und die Absolventen erlangen einen Bachelor-Abschluss, der sie als Lehrer auszeichnet. Danach besteht für Bachelors mit einer Abschlussnote von mindestens "gut" die Möglichkeit, ein kostenpflichtiges und viersemestriges Magister-Studium zu absolvieren. Neben einer Magisterarbeit findet dann auch eine mündliche Abschlussprüfung statt.

Die arbeitenden Lehrer sind in allen Schulformen in drei Qualifikationsgrade eingeteilt, bei denen die höher qualifizierten etwas mehr Gehalt bekommen (siehe zur Lehreradministration Abb. 3). Nach der Ausbildung beginnt man als Bachelor mit 
Grad III; als Magister beginnt man mit Grad II. Danach kann man bei „Erfolg“ zunächst bis zu Grad I aufsteigen. Dies wird vom sog. Bezirksleiter beurteilt. Der Bezirksleiter war vorher auch ein (Grad I-)Fachlehrer, arbeitet in der Bezirksregierung und macht in den Schulen Unterrichtsstichproben. Sportlehrer steigen nun in der Hierarchie, wenn sie Erfolge der Schulmannschaften oder -athleten vorweisen können, bei den unangemeldeten Stichproben einen strukturierten Unterricht mit entsprechenden Medien durchführen, pünktlich sind, eine adäquates Erscheinungsbild zeigen und sich im Gespräch mit dem Schuldirektor ein positives Gesamtbild ergibt. „Grad I - Lehrer“ können sich dann nach langjähriger Berufserfahrung als Bezirksleiter bewerben. Der Bezirksleiter für Sport hat neben der Lehrerbeurteilung noch weitere Aufgaben:

1. Er sorgt für eine möglichst gleichmäßige Verteilung der Lehrer auf die Schulen, wobei die Verteilung in den Mittel- und Oberschulen von einem für alle Fächer zuständigen Bezirksoberleiter vorgenommen wird. Bei einer großen Zahl von Absolventen entscheidet die Abschlussnote des Studiums.

2. Er hat bei Inventarisierung dafür zu sorgen, dass Sportstätten und Unterrichtsmaterialien in ausreichender Zahl und mit ausreichender Qualität vorhanden sind.

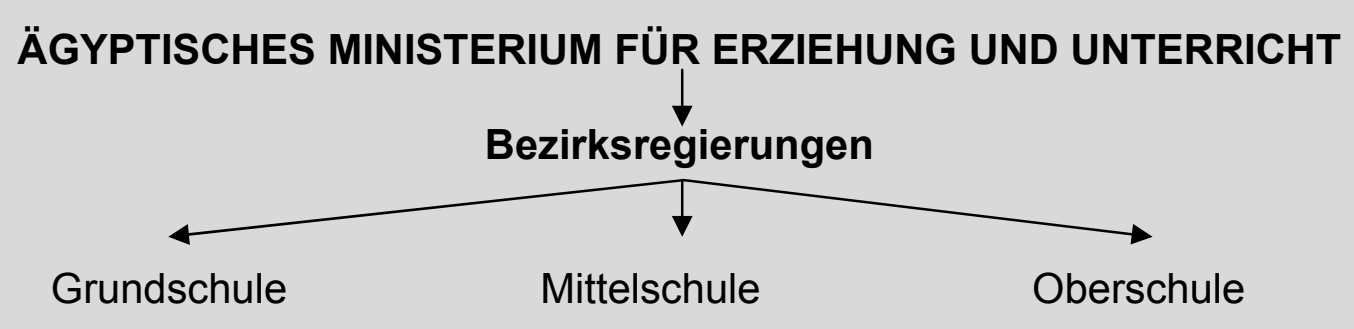

Bezirksleiter

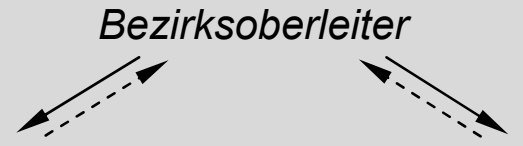

Bezirksleiter

Bezirksleiter

Grad I

Grad I

Grad I

Grad II

Grad II

Grad III

Grad III

Grad II

Grad III

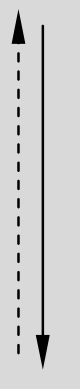

Abbildung 3: Lehreradministration in Ägypten. 


\subsection{Fazit}

Das vorliegende Kapitel zeigt anhand von einzeln ausgeführten Rahmenbedingungen die umgebende Struktur des ägyptischen Sportunterrichts. Sie wirken sich nicht nur auf den Sportunterricht aus, sondern interagieren auch miteinander. Dies sei an dieser Stelle anhand einiger markanter Eckpunkte verdeutlicht:

1. Die volkswirtschaftliche Situation Ägyptens ist durch eine hohe Staatsverschuldung und durch einen hohen Armutsstand gekennzeichnet. Ersteres limitiert die monetären Ressourcen in Bezug auf Sport und das Bildungssystem und Zweiteres die Mitgliedschaft in Sportvereinen. Deutlich wird eine geringe breitenwirksame Förderung des Sports an einer nicht vorhandenen Breitensportkultur und einer zu geringen Anzahl von Sportlehrern in den weiterführenden Schulen bei nur schlecht ausgestatteten Bildungsstätten (aus der Zeit Nassers) mit sehr großen Klassen. Allerdings besteht umgekehrt ein großes staatliches Interesse an der Förderung des Leistungssports (weil über Erfolge innenpolitische und außenpolitische Reputationen geschaffen werden sollen). Letzteres zeigt sich nicht nur an einer Förderung des Schulsports, sondern auch an der Aufnahme in die Lehrpläne. Vermutlich wirkt sich dies wiederum auf den Sportunterricht aus, weil hier einerseits Grundlagen für die Ausbildung der Sportelite geschaffen werden könnten und andererseits Sportlehrer bei ihrem Fortschreiten in der Karriere (Bezahlung nach Graden) danach beurteilt werden, ob sie sportliche Erfolge vorweisen können. Obwohl die Revolution im Juli 1952 einen politischen Wendepunkt markierte, in deren Folgezeit die Förderung von Bildung und Sport gleichberechtigt nebeneinander standen, so klafft heute eine größere Lücke zwischen Sportförderung und Bildung innerhalb des Sportunterrichts. Der hat sich zwar als Unterrichtsfach mit festen Lehrplänen etabliert, erscheint aber nicht im Zeugnis. Dementsprechend ist zu vermuten, dass Sportlehrer selten ihre Lernergebnisse durch Bewertungsmethoden überprüfen. Erschwert wird die Durchführung des Sportunterrichts schließlich durch die geografische Lage. Wenn die Wetterbdingungen ungünstig sind, findet der Sportunterricht nicht statt, weil er im Freien durchgeführt wird. 
2. Als wesentliche kulturelle Rahmenbedingungen wurden die Einflüsse von Islam als Staatsreligion und des Vaters auf das Sporttreiben - oder anders: die Einstellung zum Sport - aufgezeigt.

Während das öffentliche und staatlich geförderte Sportsystem, bei dem Fußball den höchsten Stellenwert genießt, einen Einfluss auf die Wahrnehmung und Überzeugungen von Sport hat, ist dies für den Islam in geringerem Maße festzustellen; beide verbinden mit dem Sport jedoch Wertvorstellungen wie Leistung oder Genuss, wohingegen sie sich am stärksten anhand des Wertpaares Fairness $^{12}$ - Vorbereitung für Krieg unterscheiden lassen. Abgesehen von einem indirekten Einfluss auf das Sporttreiben (über eine positive Grundhaltung) hat der Islam aber auch einen direkten Einfluss auf den Sportunterricht, wenn Ramadan ist. Dann wird das Sporttreiben - auch in der Schule - erschwert. Hinzu treten zwei weitere Aspekte. Einerseits sollten sich die stärkere Hierarchisierung in den Rollenbilder des Islam auch auf den Führungsstil des Sportlehrers auswirken (weil Gehorchen ein wichtiges Prinzip des Islam ist) und andererseits sollte sich die Zurückweisung der körperlichen Zurschaustellung (insbesondere für Frauen) auf die Kleidung beim Sporttreiben auswirken.

In Bezug auf die Familie als erste Sozialisationsinstanz des Sporttreibens wurde deutlich, dass mit dem Vater das Sporttreiben in der Freizeit und die Sichtweise von Sport steht und fällt. Da weiterhin selten Erfolge bei internationalen Wettkämpfen erzielt werden und eine gute Betreuung für Sportler fehlt (z. B. die Behandlung eines verletzten Athleten auf dessen eigene Kosten erfolgt), erscheint es für die Väter häufig wenig attraktiv, ihre Kinder Sport treiben zu lassen. Dies sollte sich neben dem Fehlen des Faches Sport auf dem Zeugnis auch auf eine geringe Bedeutsamkeitsbewertung des Sportunterrichts auswirken. Kinder werden daher eher angewiesen, sich auf die „geistigen“ Unterrichtsfächer zu konzentrieren.

Insgesamt sind damit in Bezug auf den Sportunterricht eher limitierende denn fördernde Bedingungen auszumachen, obwohl der (Leistungs-)Sport als grundsätzlich positiv angesehen wird. Im Folgenden wird der Sportunterricht selbst nun genauer zu betrachten sein.

\footnotetext{
12 Weil die Wurzeln des ägyptischen Sportsystems in der sozialistisch geprägten Zeit Nassers liegen, ist davon auszugehen, dass auch hinsichtlich des Wertekanons eine Übertragung auf den Sport stattgefunden hat.
} 


\section{Der ägyptische Lehrplan für Sportunterricht, Schul- sport und Sportlehrerausbildung}

In den ägyptischen Bildungsinstitutionen sind Sportaktivitäten mittlerweile ein wichtiger Bestandteil der Erziehung geworden. Die Rolle des in das Bildungs- und Sportsystem eingebetteten, ägyptischen Sportunterrichts besteht in der Vervollkommnung physischer, psychischer, sozialer und gesundheitlicher Aspekte, die insgesamt die Persönlichkeitsentwicklung fördern sollen (vgl. AHMAD, 1999, 11). Der Sportunterricht ist dabei jedoch primär die Basis für die Ausbildung konditioneller Aspekte und für sportartspezifische Fertigkeiten, insgesamt also einer Steigerung der physischen Leistungsfähigkeit. Daneben verspricht man sich von ihm die Bildung positiver Gewohnheiten, die Vermittlung verschiedener Kenntnisse in Bezug auf Sport und Gesundheit und dem Erlernen sozialer Fertigkeiten. Damit sind übergeordnete Erziehungsziele angesprochen, die als Leitlinien für die Gestaltung des Sportunterrichts herangezogen werden können.

Folgt man dem in der Einleitung erwähnten Analyseschema von SCHULZ (1972), so ist neben den Voraussetzungen des Unterrichts (Kapitel 2) auch dessen Feinstruktur zu betrachten. Hierzu gehören die erwähnten Erziehungsziele (die SCHULZ als Intentionen greift), Inhalte (Themen), Methoden und Hilfsmittel (Medien). Da der ägyptische Sportunterricht auf theoretischer Ebene in der ägyptischen Sportpädagogik bisher nur wenig systematisch aufgearbeitet wurde, ist es nicht verwunderlich, dass sich dies auch im „Lehrplan“ des Ministeriums für Erziehung und Unterricht zeigt ${ }^{13}$. Um nun empirische Prüfungen zu ermöglichen und dem Leser einen strukturierten Überblick zu geben, folgt dieses Kapitel der o.g. Analysestruktur, bei der im Einzelnen die Eigenschaften und Aufgaben des Sportlehrers, Erziehungsziele, Inhalte und Methodik, Rahmenbedingungen und Hilfsmittel sowie Ausbildung aufgegriffen werden. Sie werden vor dem Hintergrund der

\footnotetext{
${ }^{13}$ Weil die präzise Klärung des Begriffes Lehrplan schwer fällt, werden darunter hier ganz allgemein die Vorgaben, Forderungen und Richtlinien des Staates gefasst, aus denen sich das Wozu, Was und Wie des Sportes in der Schule ableiten lässt (vgl. BRÄUTIGAM 2003, 74 und 135). Das Besondere in Ägypten besteht darin, dass neben dem Sportunterricht auch der Schulsport und Aspekte der Lehrerausbildung aufgegriffen sind.
} 
ägyptischen Sportpädagogik und wo nötig auch der deutschen Sportpädagogik dargestellt.

\subsection{Eigenschaften und Aufgaben des Sportlehrers}

Die Lehrpläne Ägyptens (ÄGYPTISCHE MINISTERIUM FÜR ERZIEHUNG UND UNTERRICHT, 2001) $)^{14}$ beginnen zunächst mit allgemeinen Empfehlungen zu Persönlichkeitseigenschaften von Sportlehrern. Hier wird gefordert, dass Lehrer sportspezifische Kenntnisse (u.a. zur Geschichte des Sports) und Kenntnisse zur Entwicklung der Kinder und Jugendlichen haben sollte. Er soll seinen Beruf mögen und eine Führungspersönlichkeit sein. Letzteres beinhaltet neben deutlicher Sprache auch ein selbstbewusstes, freundliches, nicht nervöses und geduldiges Auftreten in sauberer Kleidung. Daneben sollte er Probleme lösen können, gesund und selbst ein guter Sportler sein. Weiterhin soll er aber auch Kulturinhalte weitergeben, fleißig sein, gesellschaftliche Probleme kennen, keine Bestechung annehmen oder Gerüchte weitertragen, die Wahrheit sprechen, mit seinen Kollegen zusammenarbeiten und Kontakte zum außerschulischen Sport pflegen. Die soziale Einbindung des Sportlehrers scheint deshalb wichtig für Sportunterricht und Schulsport, weil sich ein schlechtes Verhältnis nicht nur ungünstig auf die kooperative Verwirklichung der übergreifenden, gemeinsamen Erziehungsziele, sondern auch auf die Schulatmosphäre auswirkt. Insofern ist die Beziehung zu Kollegen keine ausschließliche Privatangelegenheit des Sportlehrers. Durch eine gute Zusammenarbeit im Kollegium können mögliche Defizite in Gesamtkonzept und Verwaltung erkannt und optimiert werden. Ist die Beziehung unter den Lehrern schlecht, so wird dies auch von den Schülern wahrgenommen. In Folge eines solchen schlechten Verhältnisses können Zweifel an der Integrität der Lehrer aufkommen, sodass deren Meinungen und Lehrinhalte - v.a. zum Sozialverhalten - nicht ihre beabsichtigte Wirkung entfalten können (vgl. ABU-HARGAH \& ZAGHLOUL, 1999, 33f).

\footnotetext{
${ }^{14}$ Die Lehrpläne für den Sportunterricht sind seit Jahren inhaltlich unverändert. Sie werden jedoch jedes Jahr mit anderem Titel und anderer Formatierung veröffentlicht. Im Literaturverzeichnis sind drei verschiedene Angaben gemacht.
} 
Seine Schüler soll der Sportlehrer gut behandeln und zum Schulsport motivieren. In besonderer Weise gilt dies für begabte Schüler. Schulsport ist aber keine zweckfreie Freizeitgestaltung, sondern sportartspezifisches Training. Hier seien dann gar psychologische und soziale Bedingungen im Wettkampf zu berücksichtigen. Grundlegend ist die Organisation und Betreuung von Schulmannschaften während des ganzen Schuljahres. Für die einzelnen Sportarten sollten schulinterne und schulübergreifende, gut strukturierte Turniere und regionale Vergleichskämpfe organisiert werden, wobei Eltern und Kollegen zum Zuschauen ermutigt werden sollen. Im Lehrplan werden konkrete Handlungsanweisungen bezüglich der Organisation gegeben. Dazu gehört die Dokumentation der Ergebnisse und Darstellungen von Turnierformen. Für das Training im Schulsport sind ebenfalls konkrete methodische Empfehlungen zu erkennen. Allerdings erfolgt die Beschreibung lediglich anhand von Bildern. In dieser Form sind im Lehrplan dann Krafttraining, Zirkeltraining, Bildreihen zu einzelnen sportspezifischen Techniken und Sportstätten (beschriftete Spielfelder mit Maßen) dargestellt.

Dem Sportlehrer fällt auch die Aufgabe zu, die Teilnahme an offiziellen Feiern (wie der Zeugnisverleihung am Ende des Schuljahres mit Eltern und Lehrern) zu unterstützen. Hierzu soll ebenfalls eine eigenständige Trainingsgruppe gebildet werden, die während dieser Feierlichkeiten Show-Einlagen präsentiert. Auch zu deren Gestaltung und Präsentation erfolgen im Lehrplan genaue methodische Handlungsanweisungen (anhand von 12 Seiten).

In Ägypten besteht aber meist eine geringe Zusammenarbeit zwischen Eltern und Schule. Oftmals kommen die Schüler den Aufforderungen der Lehrer nicht nach, weil sie zu Hause von ihren Eltern keine Unterstützung erhalten oder die Auffassungen der Eltern sich sogar gegen die entsprechenden Aufforderungen richten (z.B. auch wegen religiöser oder traditionalistischer Vorbehalte). Deshalb wird empfohlen, die Eltern-Schule-Beziehung durch einige Maßnahmen zu verbessern. Wenn die Beziehung zwischen den Eltern und der Schule gut ist, so ist dies für die Beziehung der Schüler zum Lehrer günstig, was sich vorteilhaft auf das Erlernen des Schulstoffes und auf das Selbstvertrauen der Schüler auswirkt. Probleme, die sonst verborgen bleiben, können in einer offenen, gesprächsbereiten Atmosphäre durch Mithilfe der Eltern leichter aufgedeckt und gelöst werden. Eine erste Maßnahme ist die gezielte Einladung der Eltern zu Feiern, Turnieren und sportliche Vorführungen in der Schule. Eine zweite Maßnahme besteht in persönlichen Ge- 
sprächen, die bei auftauchenden Problemen gesucht werden sollten. Hier sollte entsprechend diskutiert und deutlich gemacht werden, dass die Förderung bestimmter Fähigkeiten und Fertigkeiten bedeutsam für die weitere Entwicklung der Schüler ist (vgl. QILADA, 1998, 119f).

Vor allem soll der Sportlehrer aber Sportarten vermitteln, die im Lehrplan stehen (siehe Kapitel 3.3). Weil er für die ausgewogene Anwendung der Lehrpläne innerhalb des Schuljahres verantwortlich ist (vgl. EI HAMAHMI, \& ABD EL AZIZ, 1998, 65f), besteht eine weitere Forderung in der strukturierten und abwechslungsreichen Vermittlung von Fertigkeiten im Sportunterricht. Dies soll auf allen Könnensstufen beherrscht werden. In methodischer Hinsicht gehören dazu auch die Evaluation des Sportunterrichts und die Nutzung von Medien wie Plakaten. Daneben hat er sich um die Sportstätten und Materialien zu kümmern, sie sauber zu halten und zu inventarisieren.

An den Ausführungen wird deutlich, dass die zentrale Aufgabe des Sportlehrers in der Gestaltung von Sportunterricht und Schulsport liegen. Weil der Schulsport nun die wichtigste Reputation des Sportlehrers darstellt (auch bei seiner Beurteilung im Hinblick auf seine Karriere), ist zu vermuten, dass dies klare Auswirkungen auf die Gestaltung des Sportunterrichts hat. Mit ihm können und sollen Grundlagen für den Schulsport gelegt werden, wobei hier wieder die sportspezifische Leistungsorientierung zum Ausdruck kommt.

\subsection{Erziehungsziele}

Erst in jüngerer Zeit wurden vom Ägyptischen Ministerium für Erziehung und Unterricht neue Erziehungsziele für den ägyptischen Sportunterricht formuliert. Diese sollten als Grundlage für die Gestaltung des Unterrichts dienen. Die Ziele der Erziehung im und durch Sport lassen sich zunächst wie folgt zusammenfassen (vgl. ÄGYPTISCHES MINISTERIUM FÜR ERZIEHUNG UND UNTERRICHT, 1998, 12):

Aufgabe des Sportunterrichtes ist es, 
- die Schüler zu motivieren, ihre Freizeit durch sportliche Aktivitäten zu nutzen und ihnen die dazu nötigen Kenntnisse der Sportarten und -regeln zu vermitteln;

- den Schülern Wissen über Gesundheit zu vermitteln und die Grundlagen für einen gesunden Körper herzustellen;

- das Sozialverhalten der Schülern durch das Sporttreiben zu fördern;

- wertvolle psychische Eigenschaften der Schüler zu unterstützen;

- Freude und Spaß an der Bewegung zu vermitteln;

- den Schülern sportspezifische Kenntnisse (in erster Linie technischen und taktischen Regeln der Sportarten) zu vermitteln und

- konditionelle Fähigkeiten sowie sportartspezifische Fertigkeiten zu verbessern und einem altersentsprechenden gehoben Niveau zuzuführen.

Nun ist aber schnell erkennbar, dass die meisten dieser Erziehungsziele kaum ihre Wurzeln in der Geschichte des ägyptischen Sportsystems haben. Abgesehen von der Förderung direkter sportspezifischer Ziele treten hier völlig neue Ziele auf den Plan. Da sie sich aber auch kaum aus den kulturellen Bedingungen ableiten lassen, ist zu vermuten, dass die "Globalisierung" hier ihre Wirkung zeigt. Damit ist gemeint, dass in einem den westlichen Industrienationen gegenüber aufgeschlossenen Ägypten Ziele übernommen werden, die in Staaten wie USA oder Deutschland eine lange Entwicklungsgeschichte hinter sich haben. Letzteres bedeutet, dass solche Ziele ein gesellschaftlicher Prozess vorausgegangen ist, in dem nicht nur diese Ziele selbst ausgehandelt wurden, sondern auch ausdifferenzierte Reflexionen und Implementierungen gefolgt sind. Dies trifft auch auf den Sport in der Schule zu. Entsprechend ist zu vermuten, dass in Ägypten noch die Übertragung von Zielen in die Praxis fehlt und so erscheint es sinnvoll, die Erziehungsziele vor dem Hintergrund der ägyptischen - und wo nötig auch der deutschen - Sportpädagogik zu reflektieren. Es erscheint aber auch notwendig, weil damit ein Herunterbrechen im Hinblick auf eine Operationalisierung für die beabsichtigte empirische Prüfung erst ermöglicht wird. Im Einzelnen soll hier also zunächst ausgearbeitet werden, was die o.g. sehr abstrakten Erziehungsziele (die im Folgenden unter den Schlagworten Freizeit, Gesundheit, Sozialverhalten, psychische Eigenschaften, sportspezifische Kenntnisse und Freude / Spaß / Begeisterung zusam- 
mengefasst werden) bedeuten können; die grundlegenden sportspezifischen Fähigkeiten und Fertigkeiten sollen dabei nicht als eigene Kategorie thematisiert werden, sondern sie gehen unterschiedlich in die folgenden Kategorien ein.

\subsubsection{Freizeit}

Ein Hauptziel des Sportunterrichts ist es, zu lehren, wie die Freizeit gewinnbringend genutzt werden kann, sodass die Schüler zum selbstständigen Sporttreiben befähigt werden (vgl. KAMEL, SHALTUT \& CHAFAGA, 2002). Um diese Selbstständigkeit zu erreichen, sollen die Schüler Wissen und Können erwerben und dies entsprechend häufig üben. Dazu gehört aber auch, Werte und Normen in neuen Situationen anwenden und Formen wie Rahmenbedingungen der vorgegebenen Sportarten verändern sowie dabei weitere Fähigkeiten erwerben zu können (vgl. DIECKERT \& WOPP, 2001, 289ff). ALLAWY (1994) konkretisiert dies für Ägypten: Schüler sollen Risiken abschätzen und Sicherheitsmaßnahmen treffen können, aber bereit sein, etwas zu wagen. Sie können dabei kreativ handeln und sich schöpferisch über ihren Körper ausdrücken. Im Sportunterricht sollen dazu Grundlagen gelegt werden. Dies betrifft neben motorischen Fertigkeiten insbesondere die Vermittlung von regelgeleitetem Spielen (und hier v.a. der Sportspiele wie Fußball oder Handball). Hier steht allerdings im Vordergrund, dass die Sportarten organisiert werden können. Schüler sollen aber auch lernen, welche bewegungsbezogenen Wünsche sie haben und wie sie diesen nachgehen können. Dazu ist es hilfreich, wenn vielfältige freudvolle Erfahrungen in verschiedenen Sportarten gesammelt werden (vgl. KAMEL, SHALTUT \& CHAFAGA, 2002, 18f, s.u.).

Einteilen lassen sich die Erziehungsziele zur Freizeit nun zusammenfassend in motorische Voraussetzungen schaffen, Organisationsfähigkeit und die Anwendung dieser Merkmale in verschiedenen Sportarten. Dies ist dann die Basis für das Sporttreiben außerhalb des Sportunterrichts.

\subsubsection{Gesundheit}

Die Bedeutung von Gesundheit als Erziehungsziel des Sportunterrichts ist eine zweifache. Zum Einen geht es darum, Kenntnisse zu erwerben. Dies wiederum 
kann als Grundlage für Sportausübung in der Freizeit dienen. Insofern beinhaltet Gesundheit eine Spezifikation der Kenntnisvermittlung für die Freizeit. So soll die Bedeutung von Bewegung für das eigene Wohlbefinden im Einzelnen erkannt werden und ein Bewusstsein für körperliche Gesundheit geschaffen werden (vgl. ALLAWY, 1994). Zum Anderen soll durch das Sporttreiben selbst Gesundheit gefördert werden. Zwar wird Gesundheit heute mindestens dreidimensional betrachtet (körperliche, psychische und soziale Gesundheit; vgl. RÖTHIG, PROHL u.a. 2003), jedoch soll hier auf die körperliche Gesundheit abgehoben werden, zu der auch Hygiene gehört.

Im Zentrum des Sportunterrichts steht die Förderung körperlicher Fähigkeiten und Fertigkeiten. Diese sind auch die Grundlage für weitergehende gesundheitliche und gesellschaftliche Aspekte:

1. Ganz allgemein geht es um die Förderung der Kondition ( $\mathrm{vgl}$. im Einzelnen SPORTINSTITUT DER EL MANSOURA UNIVERSITÄT, 1998; WOLL \& BÖS, 2001, 299f). Sie lässt sich in fünf Hauptbeanspruchungsformen zerlegen: Kraft, Schnelligkeit, Ausdauer, Beweglichkeit und Koordination. Grundlage für die Förderung sind physiologische Adaptionsprozesse bzw. die Steigerung körperlicher Ressourcen, die sich in verschiedenen Körpersystemen wie Skelettmuskulatur, Nervensystem, Herz-Kreislauf-System und Hormonsystem vollziehen. Die einzelnen Aspekte lassen sich wiederum zerlegen. Beispielsweise kann es im Hinblick auf Kraft um lokale Kraftentfaltung, die Kraftausdauer oder das Zusammenspiel der Muskeln (intermuskuläre Koordination) gehen. Die einzelnen Wirkungen ergeben sich durch die praktischen Aktivitäten im Sportunterricht wie z.B. Laufen, Klettern, Werfen, Springen, Gymnastik mit Gewichten oder die Durchführung komplexer Sportarten. GRÖßING $(1997,93)$ nennt dies das Trainingskonzept der Gesundheitserziehung. In diesem Trainingskonzept wird die Verbesserung der Gesundheit über eine verbesserte Kondition angestrebt, was innerhalb der deutschen Sportpädagogik als vordergründige Gleichsetzung von Gesundheit und körperlicher Leistungsfähigkeit bemängelt wurde (vgl. FREY, 1981). Daneben stehende Konzepte wie Sicherheitserziehung (Vermeidung von Verletzungen) oder das Körpererfahrungskonzept, das auf Wohlbefinden zielt. Diese finden in Ägypten jedoch keine Berücksichtigung.

2. Die Förderung der körperlichen Aspekte erleichtert nicht nur die Lebensführung, sondern steigert auch deren Qualität. Mit einer verbesserten körperlichen 
Gesundheit werden die Schüler auf das Leben in der Gesellschaft vorbereitet. Dies betrifft in erster Linie die ökonomischen und gesellschaftlichen Dimensionen für Handwerksberufe. Damit können die Grundlagen für eine hohe Produktivität auf der Basis von Kraft, Ausdauer und Koordinationsfähigkeit geschaffen werden. Schließlich sei nochmals daran erinnert, dass der Sportunterricht entsprechende gesundheitliche Gewohnheiten aufbauen und festigen soll (u.a. wiederum die Hygiene oder Körperpflege).

Für die Untersuchung von Gesundheit scheinen vielfältige Testverfahren notwendig. Dies soll in dieser Arbeit jedoch nicht geleistet werden. Da es in erster Linie um die Implementierung der Erziehungsziele geht (und hier weniger die Effekte von Interesse sind), wird das Hauptmerkmal der Kategorie Gesundheit in den Kenntnissen gesehen und davon ausgegangen, dass sich die Förderung der körperlichen Gesundheit nebenher und quasi von selbst ergibt.

\subsubsection{Sozialverhalten}

Mit dem Sportunterricht sind in der Vergangenheit Sozialerziehung und soziales Lernen in vielfältiger Weise verbunden worden, wobei die pädagogischen Forderungen stark differierten: Von einer Erziehung zur Anpassung und Gleichschaltung bis zum Sportunterricht als Mittel zum Aufbau einer sozialistischen Gesellschaft (vgl. PÜHSE, 1990, 56 -148). Soziales Lernen bedeutet heute, dass die Abstimmung des Einzelnen mit einer sozialen Gruppe sich verbessert, indem soziale Handlungsfähigkeit erworben wird. Dabei geht es letztlich um eine Anpassung an aber auch eine Einflussnahme auf die Gesellschaft. Dieser Erwerb geschieht im Sportunterricht nicht immer absichtlich, sondern auch ungeplant, wie nebenbei (vgl. PÜHSE, 1990, 337ff).

Der Sportunterricht gibt dem Schüler nun die Möglichkeit, verschiedene Aspekte sozialer Erfahrungen wie Kooperation, Hilfeleistung, Akzeptanz oder auch Konfliktlösung zu erleben und einzuüben. Diese Kenntnisse und Fertigkeiten zeigen sich in fairem Verhalten, d.h. einerseits, sich nicht auf Kosten Schwächerer und durch rücksichtsloses Verhalten gegenüber einer Gruppe hervorzutun, sondern vielmehr zu kooperieren, und andererseits das faire Wettkämpfen, bei denen individuell optimale Ergebnisse erzielt werden sollen (vgl. ALLAWY, 1994). 
Aber auch in der Erziehungskategorie Sozialverhalten geht es wieder um den überdauernden Erwerb bestimmter Handlungsmuster für das spätere Sporttreiben in der Gruppe (vgl. KURZ, 1999) und für das alltägliche Verhalten. Unter Umständen wird auch gelernt, sich innerhalb einer Gruppe zu präsentieren oder durch entsprechendes Verhalten diese Gruppe gar zu führen, zumindest jedoch eine feste soziale Rolle zu finden und auszuüben. Weiterhin soll über den Sport und die dabei zu erwerbende Leistungsfähigkeit auch die allgemeine Leistungsbereitschaft der Schüler geweckt werden. Dem liegt der Gedanke zugrunde, dass es möglich sei, die Leistungsbereitschaft im Sport auf andere Bereiche wie Gesellschaft, Arbeit und andere Schulfächer zu übertragen. Diese Aspekte sind jedoch in hohem Maße abhängig von den Vermittlungsformen der Lehrer (vgl. BADRAN \& GHAZI, 2000, 104f). Beispielhaft sei dies für Konfliktsituationen verdeutlicht:

Konflikt treten häufiger in Wettkampfsituationen auf. Wenn Sieg oder Niederlage mit dem entsprechenden Gewinn oder Verlust an Ansehen auf dem Spiel stehen, kommt es leicht zu Auseinandersetzungen, die den weiteren Verlauf des Unterrichts behindern können. Dem Lehrer kommt dann die Rolle eines Moderators zu (vgl. CACHAY, 1980, 276 -299): Er kann beispielsweise die Situation entschärfen, indem er auch die Leistungen und gute Spielzüge der Verlierer lobt oder er dämpft generell übermäßigen Wettkampfeifer. Andererseits sollte der Lehrer bei auftretenden Differenzen diese nicht zu schnell und als übergeordneter Lenker beseitigen, denn den Schülern soll auch Gelegenheit gegeben werden, Konflikte auszuhalten und möglichst selbst zu lösen. Dazu bedarf es auch einer geordneten Konfliktlösungsstrategie, wobei der Lehrer bei weiter bestehender Uneinigkeit die Beteiligten zu Geduld, gemeinsamem Gespräch und Akzeptanz der beiderseitigen Standpunkte auffordert oder die umstrittene Situation abbricht, damit sich die Gemüter beruhigen können und später mit mehr Gelassenheit eine Einigung erzielt werden kann (vgl. BALZ, 1993, 141f).

An den Ausführungen wird deutlich, dass Sozialverhalten eng mit bestimmten Persönlichkeitsmerkmalen verbunden ist. Bestimmte psychische Bedingungen werden aber erst in der folgenden Kategorie aufgegriffen und so verbleiben für eine gezielte Untersuchung die Merkmale Konfliktlösung, Zusammenarbeit und Akzeptanz. Hieran wird deutlich, dass unter Sozialverhalten nicht das Organisieren des Sporttreibens aus dem Erziehungskomplex Freizeit gemeint ist. 


\subsubsection{Psychische Eigenschaften}

Im Sportunterricht können Erfahrungen wie z.B. Anerkennung durch Leistung gemacht werden. Dies kann motivieren, den Sport auch nach der Schulzeit auszuüben oder gar zu einer leistungsbetonten Gesamthaltung zu finden (vgl. ALLAWY, 1993, 76f). Dazu ist es notwendig, dass die motorische und konditionelle Leistungsfähigkeit verbessert wird und diese individuell eingeschätzt werden kann. Darüber sollen Schüler lernen, dass Leistung ein lohnenswertes Ziel ist und Anstrengungen zum Erreichen notwendig sind (vgl. ALLAWY, 1994). Die individuelle Einschätzung ist aber auch an Zutrauen gebunden. Wenn sich eine spezifische Aufgabenstellung ergibt, liegt eine wesentliche Voraussetzung darin, dass der Schüler die Überzeugung hat, dass er die Aufgabe auch lösen kann. Im deutschsprachigen Raum wird dies mit dem Konstrukt der Selbstwirksamkeit gefasst (vgl. z.B. BUND 2001), wird in Ägypten jedoch nicht differenziert thematisiert. Recht allgemein nennt NAGLA $(1993,23 f)$ daher als wichtige Ziele des Sportunterrichts die Stärkung von Kampfgeist (wodurch Leistung und Anstrengung zum Ausdruck kommen) und von Selbstvertrauen (vgl. auch ganz ähnlich EL CHOULI, ABD EL FATTAH \& DARWISH, 1994, 23f; ABD EL AZIEM, 1993). Eine solche Förderung ist aber nur möglich, wenn die individuellen Leistungsniveaus von Fähigkeiten und Fertigkeiten im Unterricht berücksichtigt werden.

Eine ähnliche Argumentation (überdauernde Eigenschaft - positive Erfahrungen individuelles Niveau als Ansatzpunkt) lässt sich auch auf Führungsqualitäten übertragen, denn dies gehört ebenso zu explizit gewünschten Erziehungszielen (vgl. ÄGYPTISCHES MINISTERIUM FÜR ERZIEHUNG UND UNTERRICHT, 1998). Hier spielen jedoch Aspekte des Sozialverhaltens (s.o.) die tragende Rolle. Weil die Erziehungsziele individuelle Leistung und Zusammenarbeit allerdings teilweise in Konflikt stehen können, erfordert dies ausdifferenzierte Konzepte, die den Lehrer solche Zielkonflikte lösen lassen können. Dies ist in Ägypten jedoch bisher nicht erfolgt. Vielmehr wird auf ein in dieser Situation hilfreiches Merkmal verwiesen, dass seine Wurzeln im Islam hat, nämlich dem Gehorsam.

Damit lassen sich als wünschenswerte psychische Eigenschaften, die der ägyptische Sportunterricht fördern soll, nennen: Selbstvertrauen, Kampfgeist, Führungsqualitäten und Gehorsam. 


\subsubsection{Sportspezifische Kenntnisse}

Sportspezifische Kenntnisse erscheinen bedeutend, denn auf dieser Grundlage sollen die Schüler lernen, wie sie praxisorientiert Probleme lösen können (vgl. ALLAWY, 1994). Entsprechend entstehen mit der Zeit möglichst individuelle Strategien, die auch im späteren Leben Anwendung finden können. Die Verknüpfung von Wissen und Problemlösungen kann im Sportunterricht leicht eingeübt werden - und dies auf einem Gebiet, das Spaß macht. Allerdings liegt hier wieder eine Konkretisierung aus dem Erziehungsbereich Freizeit vor. Hier geht es nicht mehr nur um die Organisation des Sporttreibens, sondern vielmehr um seine regelgeleitete Ausstaffierung. Dies betrifft neben Regeln von Sportarten (in Bezug auf Spielidee/Ziel, Name, Beschaffenheit und Umgang mit Sportgeräten und Kenntnisse über die Sportstätten wie Maße von Spielfeldern) auch taktische Regeln. Dazu gehören beispielsweise die Abstimmung auf einen Gegner oder Mitspieler, die Wahrnehmungslenkung oder die strategische Denkfähigkeit, sodass der Sportunterricht einen großen Einfluss auf die geistige Entwicklung der Schüler ausübt (vgl. ÄGYPTISCHES MINISTERIUM FÜR ERZIEHUNG UND UNTERRICHT, 1996, 33).

Der Sportunterricht ermöglicht dabei die Entdeckung der individuellen sportlichen Neigungen und Begabung. Zu Wissen, was man kann und möchte, ist eine wichtige Bedingung für zukünftiges Sporttreiben. Daher ist es notwendig, möglichst viele Sportarten auszuüben und über diese Sportarten Bescheid zu wissen (vgl. ALLAWY, 1994, s.u.). Ein möglicher Weg zur Förderung von Interesse liegt darin, den Schülern von nationalen und Internationalen Sportereignissen zu berichten. Zusammenfassend lassen sich als Teilziele der sportspezifischen Kenntnisse Regeln, Sportereignisse und vielfältige Erfahrungen nennen.

\subsubsection{Spaß/Freude/Begeisterung}

Wie erwähnt, ist während des Unterrichts ein vielfältiges Üben und Spielen anzustreben; dabei ist möglichst die Leistungsfähigkeit der einzelnen Schüler zu berücksichtigen, um allen Schüler die Teilnahme und Erfolge zu ermöglichen und 
Freude an der Bewegung zu erhalten oder zu fördern (vgl. ALLAWY, 1994). Letzteres wird als Grundlage für ein Sporttreiben über die Schulzeit hinaus betrachtet. Spaß und Freude am oder Begeisterung für den Sport als eigenständige Kategorie von Erziehungszielen ist ebenfalls mit der Kategorie Freizeit aber auch mit Sozialverhalten verknüpft. Es wird aber schnell deutlich, dass sie vielmehr mit psychischen Eigenschaften verbunden sind, denn bei der Umsetzung von Lehrplänen sind die Bedürfnisse und Neigungen der Schüler zu berücksichtigen (vgl. NAGLA, 1993; EL CHOULI, ABD EL FATTAH \& DARWISH, 1994, 23f). Hier wird allerdings vorausgesetzt, dass die Befriedigung von Bedürfnissen zwangsläufig zu Spaß oder Freude an der Aktivität führt. Dabei stellt sich die schwierige Frage, worin solche Bedürfnisse liegen können. In jüngster Zeit legte DAHLHAUS (2004) dazu eine Konzeption vor, die sich in einem Punkt mit den zuvor genannten ägyptischen Autoren deckt: Anerkennung (beispielsweise für Leistungen) wird als ein solches Bedürfnis genannt. Wesentlich an der Konzeption von DAHLHAUS ist aber, dass die Befriedigung der Bedürfnisse in hohem Maße von dem Interaktionspartner abhängig ist. Überträgt man dies auf den Sportunterricht, so bedeutet dies, dass das Verhalten des Lehrers ausschlaggebend für Freude oder Spaß ist. Zunächst betrifft dies bestimmte Rückmeldungen. Hier wären nicht nur Fehler zu korrigieren, sondern auch gute Leistungen zu loben (Anerkennung). Ein weiteres Merkmal sind die Hilfen, die der Sportlehrer gibt. Wenn eine bestimmte, zuvor misslungene Bewegung durch eine solche Hilfe klappt, ist dies sicher eine freudvolle Erfahrung. Neben den Bedürfnissen (die hier nun vereinfachend auf konkrete Wünsche der Schüler bezogen werden), ist aber eine Begeisterung für den Sport zu wecken. Diese kann sich aus dem Vorbildcharakters des Sportlehrers ergeben. Insofern scheinen für die Kategorie Freude/Spaß/Begeisterung zusammenfassend Rückmeldung, Hilfe, Bedürfnisse und Vorbild von Bedeutung. 


\subsubsection{Zusammenfassung und Schlussfolgerungen}

Bei der komplexen Zielstellung der Erziehung im, durch und zum Sport sind verschiedene übergeordnete Kategorien wie motorische Aspekte, Gesundheit, Kenntnisvermittlung, psychische Eigenschaften und soziale Fertigkeiten erkennbar. In Bezug auf die Sportdidaktik lassen sich darauf zwar unterschiedliche Perspektiven werfen (vgl. dazu beispielsweise BECKER, 1998; BRÄUTIGAM, 2003; GRÖßING, 1997), jedoch steht das Sporttreiben selbst sowohl im deutschen als auch im ägyptischen Sportunterricht im Vordergrund. Die Erfahrungen, die dort gemacht werden, dienen nicht nur einem Sporttreiben über die Schulzeit hinaus, sondern bereichern auch die Schulkultur. In Wettkämpfen können Leistungen erbracht und Anerkennung erworben werden; sportspezifische Fähigkeiten und Fertigkeiten, Kompetenzen und Kenntnisse sollen vermittelt werden. Die Gesundheit bzw. Fitness soll mit der Durchführung verschiedener Sportarten aktuell gefördert werden, Kenntnisse und ein Bewusstsein für den verantwortungsvollen Umgang mit dem eigenen Körper erworben werden. Schließlich soll er noch fachübergreifende Beiträge liefern, indem er prosoziales Verhalten und Persönlichkeitseigenschaften unterstützt.

In der deutschen Sportpädagogik wird deshalb von einem mehrperspektivischen Sportunterricht gesprochen (vgl. KURZ, 1999). Dabei werden den Schülerinnen und Schülern möglichst die verschiedenen Sinnrichtungen des sportlichen Handelns zugänglich gemacht und mit ihrer persönlichen Erfahrungs- und Erlebniswelt verknüpft. Die Inhalte des Sportunterrichts dienen als Medium, um verschiedene übergeordnete Zielbereiche zu erlangen. Im Sportunterricht darf es demnach also nicht nur um bloßen Bewegungsvollzug gehen, sondern auch um die Gewinnung von Erkenntnis, um Reflexion des Gegenstandes und der Methode, um selbständiges Lösen-Können von Problemen. Zu einer intensiven und reflektierten Auseinandersetzung mit dem Handlungsfeld Sport gehört nicht nur, dass die Schüler lernen, ihr sportliches Tun selbständig planend und gestaltend einzurichten und zu verbessern, sondern sie sollen sich vielmehr auch ein Bild von der vielfältigen Sportwirklichkeit machen können und erfahren, welche gesellschaftlichen Aspekte 
mit dem Sporttreiben verbunden sind, sodass die Schüler (überdauernd) in einem Lebensbereich verantwortlich handeln und inn mitgestalten können ${ }^{15}$.

Doch vor dem Hintergrund der unterschiedlichen Kultur (und insbesondere des Sport- und Schulsystems, siehe Kapitel 2) erhalten diese Zielbereiche in Ägypten andere Schwerpunkte. Sicherlich besteht auch in Deutschland ein staatliches Interesse an der Gesundheitsförderung sowie an einem Sporttreiben „über die Schulzeit hinaus“, jedoch zeigt sich in Bezug auf Letzteres der größte Unterschied. Während der deutsche Sportunterricht primär das Sporttreiben nach der Abschlussprüfung einer Schulform im Blick hat, rückt im ägyptischen Sportunterricht der Schulsport ins Zentrum des Interesses, denn "der Sportunterricht soll zur Entwicklung des Sports in allen Aspekten beitragen" (vgl. ÄGYPTISCHES MINISTERIUM FÜR ERZIEHUNG UND UNTERRICHT, 1991,17). Ruft man sich in Erinnerung, dass dieser leistungssportlich orientiert (und ohne ausgeprägte Breitensportkultur) ist, so wird deutlich, dass mit dem Schulsport die Basis für die Sportelite geschaffen werden soll. Nur der Sportelite und einigen Wohlhabenden im Vereinssport ist das Sporttreiben dann im Erwachsenenalter vorbehalten. Im Sinne einer Vorauswahl - und einer zeitlich kürzeren Verständnis von „über die Schulzeit hinaus" - soll der ägyptische Sportunterricht den Schulsport befruchten. Also: Sportunterricht als Basis des Schulsports und Schulsport als Basis für die sportliche Elite im Leistungssport sowie für die Wohlhabenden im Vereinssport. Vor diesem Hintergrund werden dann selbstverständlich auch soziale und psychische Ziele genannt, jedoch ist zu bezweifeln, dass sie mit den gleichen Wertvorstellungen wie in Deutschland auch in Bezug auf den Sport gefüllt werden sollen. So lassen sich die übergeordneten Erziehungsziele von KAMEL, SHALTUT \& CHAFAGA (2002) - Freizeit, Begeisterung, Psychosoziale Ziele und Kenntnisse ganz unterschiedlich lesen. Auch die Empfehlungen von KAMAL (2002) ergeben einen anderen Sinn, wenn man beim Lesen anstatt dem Oberziel einer ausdifferenzierten Breitensportkultur,Schulsport - Leistungssport/Clubsport' einsetzt:

\footnotetext{
${ }^{15}$ Dem Sportunterricht in der Gewerbeschule stellt sich beispielsweise die Aufgabe, den Erfahrungsgehalt und die Erziehungsziele des Faches Sport mit den Wünschen der Schülerinnen und Schüler, vor dem Hintergrund der besonderen Bedingungsfaktoren in Einklang zu bringen (vgl. ABD EL-KARIM, 1999). Darauf sei aber hier im Einzelnen nicht eingegangen.
} 
- Der Sportunterricht soll den Schülern möglichst vielfältige körperliche Erfahrungen vermitteln und sie über deren Reflexion zu einer eigenverantwortlichen Ausbildung der Körperlichkeit befähigen.

- Der Sportunterricht soll die Schüler anleiten, die Rahmenbedingungen und den Ablauf ihres Sporttreibens sowie sportlicher Übungs- und Wettkampfsituationen zunehmend selbst zu gestalten und zu verantworten.

- Der Sportunterricht soll die Schüler über die Auseinandersetzungen mit der vielfältigen Sportwirklichkeit befähigen, am Sport außerhalb der Schule teilzunehmen und inn mitzugestalten.

- Der Sportunterricht soll die Gesundheit der Schüler fördern; er soll sportbezogene Kenntnisse einzelner Sportarten, Einsichten und Gewohnheiten ausbilden, die eine gesunde Lebensführung in persönlicher und sozialer Verantwortung stützen können.

- Der Sportunterricht soll die Schüler befähigen, an sportlichen, von Regeln geleiteten Spielen unterschiedlicher Komplexität handlungstragend teilzunehmen; er soll zugleich ein Regelbewusstsein fördern, das an der Idee orientiert ist, alle in die Spielhandlung einzubeziehen.

- Der Sportunterricht soll den Schülern vielfältige, den jeweiligen Voraussetzungen angemessene Möglichkeiten bieten, Leistungen zu vollbringen und innen bewusst machen, dass und wie ihre Leistungsfähigkeit zu verbessern ist; er soll sie auch anleiten, die Kriterien, nach denen im Sport Handlungen als Leistungen bewertet werden, zu verstehen und situativ zu verändern.

Für die empirische Prüfung der Implementierung von Erziehungszielen im ägyptischen Sportunterricht ergeben sich schließlich zusammenfassend folgende Kategorien:

1. Freizeit: Motorische Voraussetzungen, Organisationsfähigkeit, verschiedene Sportarten.

2. Gesundheit: Kenntnisse.

3. Sozialverhalten: Konfliktlösung, Zusammenarbeit, Akzeptanz.

4. Psychische Eigenschaften: Selbstvertrauen, Kampfgeist, Führungsqualitäten, Gehorsam.

5. Sportspezifische Kenntnisse: Regeln, Sportereignisse, vielfältige Erfahrungen.

6. Spaß/Freude/Begeisterung: Rückmeldung, Hilfe, Bedürfnisse, Vorbild. 


\subsection{Inhalte und Methodik}

Die zuvor genannten Ziele sind grundsätzlich durch eine gute Planung und Durchführung des Sportunterrichts zu verwirklichen, indem alle vorhandenen materiellen und personellen Möglichkeiten ausgeschöpft werden. Die bereits geschilderten Probleme, wie große Klassen oder das Fehlen von Materialien, erschweren dies jedoch in starkem Maße. Umso wichtiger erscheint daher das methodischdidaktische Vorgehen während des Unterrichts, zu denen hier auch die Inhalte sowie deren Jahresstruktur gezählt werden.

\subsubsection{Inhalte und übergeordnete (Jahres-)Strukturen}

Die ägyptischen Lehrpläne versuchen den kindlichen und jugendlichen Entwicklungsstadien zu werden. Dies bedeutet, dass zunehmend komplexere und sportspezifischere Fertigkeiten unterrichtet werden. Auch das individuelle oder klassenkollektive Niveau von Fähigkeiten und Fertigkeiten soll berücksichtigt werden. Ebenso soll sich die Vermittlung der Kenntnisse am intellektuellen Entwicklungsstand orientieren. Während in der Grundschule für acht Monate im Jahr Sport unterrichtet wird, wird in der Mittel- und Oberschule nur noch sechs Monate Sportunterricht durchgeführt.

\section{Grundschule}

Folgende Tabelle (6) soll verdeutlichen, dass der Sportunterricht in der Grundschule auch motorische Grundfertigkeiten vermitteln soll. Wie im Rahmen der Schulsystems (Kapitel 2.4.2) schon erwähnt, sind die ersten drei Jahre der Schule ein Abschnitt, bei dem grundlegende Fertigkeiten gelehrt werden sollen. Mit einem spielerischen Bewegen sollen Interesse für das Fach Sport geweckt werden. Es schafft zunehmend Koordination, Körperausdruck und grundlegende turnerische wie leichtathletische Fertigkeiten. Das jährliche Unterrichtskonzept hat in den ersten drei Jahren die gleiche Struktur. 


\begin{tabular}{|c|c|c|c|}
\hline Monat & 1. Klasse & 2. Klasse & 3. Klasse \\
\hline $\begin{array}{l}\text { Septem- } \\
\text { ber }\end{array}$ & Zirkusbesuch & $\begin{array}{l}\text { Spiele zur Förderung } \\
\text { der Auge-Hand- und } \\
\text { Auge-Fuß-Koordination }\end{array}$ & $\begin{array}{l}\text { Bewegungsgeschichten } \\
\text { (Ausdrucksspiele) }\end{array}$ \\
\hline Oktober & $\begin{array}{l}\text { Bewegungsgeschich- } \\
\text { ten (Ausdrucksspiele) }\end{array}$ & Gleichgewichtsübungen & Variation dieser Spiele \\
\hline $\begin{array}{l}\text { Novem- } \\
\text { ber }\end{array}$ & Ballspielen & $\begin{array}{l}\text { Verschiedene Lauffor- } \\
\text { men (Hopserlauf, Si- } \\
\text { desteps, u.a.) }\end{array}$ & $\begin{array}{l}\text { Verschiedene Lauffor- } \\
\text { men (Hopserlauf, Si- } \\
\text { desteps, u.a.) }\end{array}$ \\
\hline $\begin{array}{l}\text { Dezem- } \\
\text { ber }\end{array}$ & Werfen und Fangen & $\begin{array}{l}\text { Bewegungsgeschichten } \\
\text { (Ausdrucksspiele), } \\
\text { Handstand mit Hilfestel- } \\
\text { lung }\end{array}$ & $\begin{array}{l}\text { Üben von Koordination } \\
\text { und Timing in unter- } \\
\text { schiedlichen Bewegun- } \\
\text { gen, Gymnastik für } \\
\text { Kraft, Beweglichkeit } \\
\text { und Gleichgewicht, so- } \\
\text { wie Schnelligkeit (Part- } \\
\text { nerübungen), }\end{array}$ \\
\hline Januar & $\begin{array}{l}\text { wie vorher, mit Wand- } \\
\text { kontakt }\end{array}$ & $\begin{array}{l}\text { Rad schlagen mit Hilfe- } \\
\text { stellung }\end{array}$ & $\begin{array}{l}\text { Rolle rückwärts, Waa- } \\
\text { ge, Brücke }\end{array}$ \\
\hline Februar & $\begin{array}{l}\text { Springen und Laufen } \\
\text { mit Rhythmus }\end{array}$ & $\begin{array}{l}\text { Laufen auf allen Vieren } \\
\text { und Weitsprung }\end{array}$ & Weitsprung, Staffellauf \\
\hline März & $\begin{array}{l}\text { Laufen mit Seil und } \\
\text { Rhythmus, Ballspiel, } \\
\text { Koordinationsgymnastik, } \\
\text { Seilspringen }\end{array}$ & $\begin{array}{l}\text { Rhythmische Übungen } \\
\text { mit und ohne Musik }\end{array}$ & $\begin{array}{l}\text { Rhythmische Übungen, } \\
\text { Werfen und Fangen, } \\
\text { Kleine Spiele }\end{array}$ \\
\hline April & $\begin{array}{l}\text { Ballwurf und Reifenspie- } \\
\text { le }\end{array}$ & $\begin{array}{l}\text { Verschiedene Lauffor- } \\
\text { men (Hopserlauf, Si- } \\
\text { desteps, u.a.) }\end{array}$ & Spiele mit Ball \\
\hline
\end{tabular}

Tabelle 6: Lehrinhalte des Sportunterrichts in den ersten drei Klassen der Grundschule (Quelle: ÄGYPTISCHES MINISTERIUM FÜR ERZIEHUNG UND UNTERRICHT, 2001).

Ab Klasse vier ändert sich die Lehrplan- bzw. Unterrichtsstruktur. Von nun an werden in einer Unterrichtseinheit (siehe Kapitel 3.3.2) zwei Hauptteile unterrichtet: Gymnastik zur Schulung der konditionellen Fähigkeiten und eine Sportart. Dazu gehören die Individualsportarten Turnen und Leichtathletik sowie die Spielsportarten Fußball, Handball, Basketball und Volleyball. Diese Struktur und diese Inhalte werden dann die ganze Schulzeit beibehalten, also auch in Mittel- und Oberschule so unterrichtet. Bei zunächst freier Auswahl der Inhalte, was Zeitperioden betrifft, werden in den einzelnen Sportarten folgende Inhalte vermittelt: 
- Gymnastik: freies Aufwärmen/Spielen, angeleitete (Kleine) Spiele, Kreisspiele.

- Turnen: Gleichgewichtsübungen (z.B. Waage, Laufen in der Luft nach Hochspringen), Handstand, Brücke, Rolle vor-, seit- und rückwärts.

- Leichtathletik: 30m-Lauf, Weitsprung, Ballweitwurf (300g), Stabwechsel (Staffellauf), Wettkampf.

- Fußball: Bewegung mit Ball, Pässe, Dribbling, Ballkontrolle, Torschuss, Körpertäuschung mit Ball und Spielregeln.

- Basketball: Ball führen (prellen), Passen und Fangen, Pässe aus der Bewegung, Zielwerfen auf den Korb, Dribbeln mit Handwechsel und Spielregeln.

- Handball: Werfen und Fangen aus dem Stand und in Bewegung, Grundhaltungen in der Verteidigung, Schlagwurf mit Stemmschritt, Wurftäuschungen und Spielregeln.

- Volleyball : Pritschen (Paarweise), Spielen auf dem Kleinspielfeld, Ballannahme durch Fangen, Baggern über eine Schnur nach Aufschlag von unten, Spielregeln.

Eine zusätzliche Strukturierung erhält der Sportunterricht ab Klasse 5. Auch diese Struktur wird bis zum Ende der Schulzeit beibehalten: Vier unterschiedlich zusammengesetzte Unterrichtsblöcke werden sechs Wochen lang unterrichtet. Die Blöcke setzen sich aus einem gymnastischen Teil, der die konditionellen Fähigkeiten schulen soll, einem Teil Individualsportart (Leichtathletik oder Turnen) und einem Teil Spielsportart zusammen. Da die grundlegende Struktur aus der vierten Klasse beibehalten wird, bedeutet dies, dass Gymnastik in jeder Unterrichtseinheit durchgeführt wird und dann eine weitere Sportart erlernt wird. Nachfolgende Tabelle zeigt den aktuellen Lehrplan für Klasse 5, wobei ersichtlich ist, dass die Gymnastik immer dem gleichen Schema folgt und die Individualsportarten deutlich schwerer gewichtet werden. Obwohl daraus zunächst zu folgern ist, dass in einem Unterrichtsblock neben Gymnastik jede Sportart drei Wochen unterrichtet wird, ist dies nicht der Fall, denn die Dauer der Sportartvermittlung bleibt dem Lehrer (bzw. seinen Neigungen) überlassen. 


\begin{tabular}{|c|c|c|c|c|c|c|c|}
\hline \multirow{2}{*}{$\begin{array}{r}\text { Sport- } \\
\text { arten }\end{array}$} & \multirow[t]{2}{*}{ Gymnastik } & \multicolumn{2}{|c|}{ Individualsportarten } & \multicolumn{4}{|c|}{ Spielsportarten } \\
\hline & & $\begin{array}{l}\text { Leicht- } \\
\text { athletik }\end{array}$ & Turnen & Fußball & Handball & $\begin{array}{c}\text { Basket- } \\
\text { ball }\end{array}$ & $\begin{array}{l}\text { Volley- } \\
\text { ball }\end{array}$ \\
\hline A & $\begin{array}{l}\text { Gymnastische } \\
\text { Übungen zur } \\
\text { Steigerung } \\
\text { der konditio- } \\
\text { nellen Fähig- } \\
\text { keiten, darun- } \\
\text { ter auch } \\
\text { rhythmische } \\
\text { Übungen und } \\
\text { verschiedene } \\
\text { Laufformen }\end{array}$ & $\begin{array}{l}30 \text { m-Lauf mit } \\
\text { Tiefstart, } \\
\text { Weitsprung } \\
\text { mit Ab- } \\
\text { sprungbal- } \\
\text { ken, Ball- } \\
\text { weitwurf, } \\
\text { Staffellauf, } \\
\text { Wettkampf }\end{array}$ & - & - & - & - & $\begin{array}{l}\text { Aufschlag } \\
\text { von un- } \\
\text { ten, Prit- } \\
\text { schen, } \\
\text { Schmet- } \\
\text { terball, } \\
\text { Spielre- } \\
\text { geln und } \\
\text { Spiel }\end{array}$ \\
\hline B & wie oben & $\begin{array}{l}\text { Weitsprung, } \\
\text { Ballweitwurf, } \\
\text { Gewöh- } \\
\text { nungsübun- } \\
\text { gen mit der } \\
\text { Kugel }\end{array}$ & - & - & - & $\begin{array}{l}\text { Fangen, } \\
\text { Dribbeln } \\
\text { mit } \\
\text { Hand- } \\
\text { wechsel } \\
\text { im Stand } \\
\text { und in } \\
\text { der Be- } \\
\text { wegung, } \\
\text { regelge- } \\
\text { rechtes } \\
\text { Dribbeln }\end{array}$ & - \\
\hline C & wie oben & - & $\begin{array}{l}\text { Handstand, } \\
\text { Rolle vor- } \\
\text { wärts und } \\
\text { rückwärts, } \\
\text { Gleichge- } \\
\text { wichtsü- } \\
\text { bungen wie } \\
\text { Waage }\end{array}$ & $\begin{array}{l}\text { Torschuss, } \\
\text { kleine } \\
\text { Fußball- } \\
\text { wettkämp- } \\
\text { fe, } \\
\text { Körpertäu- } \\
\text { schung mit } \\
\text { Ball }\end{array}$ & - & - & - \\
\hline D & wie oben & - & wie oben & - & $\begin{array}{l}\text { Aus dem } \\
\text { Prellen } \\
\text { Würfe } \\
\text { aufs Tor, } \\
\text { Werfen/ } \\
\text { Passen } \\
\text { und Fan- } \\
\text { gen im } \\
\text { Stand } \\
\text { und in } \\
\text { Bewe- } \\
\text { gung, } \\
\text { Dribbeln } \\
\text { mit } \\
\text { Hand- } \\
\text { wechsel, } \\
\text { Spiel }\end{array}$ & - & - \\
\hline
\end{tabular}

Tabelle 7: Unterrichtsinhalte der 5. Klasse in den sechswöchigen Unterrichtsblöcken (Quelle: ÄGYPTISCHES MINISTERIUM FÜR ERZIEHUNG UND UNTERRICHT, 2001). 


\section{Mittel- und Oberschule}

Für die Mittel- und Oberschule werden im Lehrplan dann lediglich noch Angaben zu den Fertigkeiten und Unterrichtsinhalten gemacht, die in den einzelnen Sportarten vermittelt werden sollen. Beispielhaft sei dies anhand der 2. Klasse der Mittelschule (bzw. der 7. Klasse) verdeutlicht:

- Leichtathletik: Laufen mit gleichmäßiger Schrittlänge, Läufe zwischen 3 und 5 Minuten, Skippings/Kniehebelauf, Tiefstart, Hochsprung, Speerwurf, Wettkampf (Mehrkampf), Bewertung.

- Turnen: Rolle vorwärts aus dem Stand, Gleichgewichtsübungen (z.B. Waage), Kopfstand mit Abrollen, Zusammensetzung der zuvor genannten Elemente in einer Übung.

- Basketball: Beid- und einhändige sowie direkte und indirekte Pässe, Distanzpässe, Werfen auf den Korb, Dribbeln mit rechter und linker Hand, Wettkampf (Spiel nach Regeln)

- Volleyball: Zuspiel von oben und unten (Pritschen und Baggern), Wettkampf (Spiel nach Regeln), Bewertung.

- Handball: Werfen und Fangen in verschiedenen Höhen, Torwürfe, Dribbeln, Wettkampf (Spiel nach Regeln), Bewertung.

- Fußball: Pass und Passannahme, Doppelpässe, Eckbälle, Ballkontrolle, Körpertäuschung mit Rhythmus, Wettkampf (Spiel nach Regeln), Bewertung.

Ersichtlich wird an der Auflistung, dass in den weiterführenden Schulen nicht mehr nur Fertigkeitsvermittlung stattfindet, sondern vielmehr auch trainiert wird. Zusätzliche Momente sind die Bewertung der meisten Sportart (nicht jedoch Gymnastik, Turnen und Basketball), die Anwendung der Fertigkeiten in Wettkämpfen und nach der 7. Klasse werden noch taktische Elemente der Sportspiele aufgegriffen. In der Abschlussklasse werden dann alle behandelten Fertigkeiten trainiert, überprüft und im Wettkampf angewandt. 


\subsubsection{Struktur einer Unterrichtseinheit}

Der Lehrplan des ägyptischen Sportunterrichts ist die Richtlinie, anhand der die allgemeinen Ziele des Sportunterrichts erreicht werden und die für die Unterrichtsplanung des Lehrers herangezogen werden sollen. Der Lehrplan beinhaltet daher in erster Linie organisatorische Empfehlungen, die den Unterrichtsablauf und das Erreichen der Erziehungsziele unterstützen sollen (vgl. EL HAMAHMI \& EL CHOULI, 1990, 28f). Auch ABD EL MAWGUUD (1995, 87f) weist darauf hin, dass gerade die übergeordnete Organisation des Sportunterrichts eine der wichtigsten Bedingungen für die Gewährleistung eines gelungenen Unterrichts ist. Sie wirkt sich positiv auf die Unterrichtsatmosphäre sowie auf die Beziehung zwischen Lehrer und Schüler aus und soll die Interaktion unter den Schülern fördern. Eine gute Unterrichtsorganisation trägt dazu bei, dass die Schüler die Fähigkeit erwerben, den Unterricht adäquat zu erleben und zu verstehen. Sie hilft den Schülern, ihr Verhalten positiv zu gestalten, sodass sie eine erwünschte Verhaltensnorm erwerben. Im Gegensatz dazu wirkt sich die Vernachlässigung der Unterrichtsstruktur nachteilig auf die Konzentration der Schüler während des Unterrichts aus, was effektivem Handeln entgegensteht. Damit geht der Nutzen des Sportunterrichts zu einem großen Teil verloren.

$\mathrm{Zu}$ einer organisierten Gestaltung wird der Sportunterricht nun in einzelne Abschnitte zerlegt. Neben der inhaltlichen Ausgestaltung und der Erledigung einiger übergreifender Vorbereitungen, gehört zum Sportunterricht als erster Abschnitt die direkte Vorbereitung, womit insbesondere die Kontrolle darüber gemeint ist, ob die Geräte und der Platz in adäquatem Zustand sind, ob die notwendigen Geräte (in der Nähe des Sportplatzes) vorhanden sind und ob die Sportplätze für die Aktivitäten präpariert worden sind. Dies muss auch im Interesse der Sicherheit der Schüler gewährleistet sein. Die Sportunterrichtzeit in Ägypten beträgt dann zwei Mal in der Woche 45 Minuten, insgesamt 90 Minuten. Für die Durchführung des Unterrichts formuliert HUMMUS $(1997,30)$ eine festgelegte, relativ rigide Struktur, die die Umsetzung erleichtern soll und die in Ägypten in der Praxis auch üblich ist:

1. Treffen des Lehrers mit den Schülern.

2. Umkleiden (Wechsel der Alltagsbekleidung in Sportbekleidung).

3. Gemeinsames Aufsuchen des Trainingsplatzes.

4. Aufstellung in Reihen und Kontrolle der Anwesenheit inkl. Dokumentation. 
5. Sportpraktischer Teil (s.u.).

6. Aufstellung in Reihen und abschließende Besprechung.

7. Reinigung im Waschraum und Umkleiden.

8. Gemeinsames Zurückgehen zu den Klasseräumen.

Der sportpraktische Teil des Sportunterrichts besteht aus vier Hauptabschnitten. Diese starre Struktur soll immer verfolgt werden:

\section{Stundenbeginn}

Dieser Teil soll ca. 7 Minuten dauern. Er besteht aus:

a - Verwaltungsaufgaben

1. Hierzu gehören die o.g. Punkte 1-4 von HUMMUS (1997).

2. Die Vorbereitung der Mittel und der Geräte für den Unterricht (vgl. EL LAQANI, 1995, 37f).

$b$ - Aufwärmen

Das Aufwärmen gilt als der wirkliche Beginn des Unterrichts, weshalb es mit der Anregung des sportlichen Konkurrenzgedankens gekennzeichnet sein soll. In diesem Teil werden Laufen, Springen und gemeinsamen Spiele bevorzugt. Die Schüler sollen sich bei freier Auswahl der Übung aufwärmen. Das Aufwärmen hat die folgenden Ziele:

1. Es aktiviert das Nervsystems, die Muskel und die vitalen Organe des Körpers.

2. Es hilft, die Schüler vor Verletzungen zu schützen.

3. Es bereitet die Schüler psychisch auf die anderen Teile des Unterrichts vor.

Die gebräuchliche Aufwärmungsart ist bei Jüngeren freies Spiel und bei den Älteren Spiel oder Gymnastik, jeweils mit oder ohne Hilfsmittel / Geräte (vgl. ABD EL KARIM, 1999, 28f). Dabei kann das Aufwärmen auch als Einleitung für den vorgesehen Hauptteil erfolgen, wodurch vermieden wird, dass auf eine möglicherweise die Schüler mehr ansprechende Aufwärmübung ein weniger reizvoller Unterrichtsteil folgt. Eventuell kann auf ein spezielles Warmmachen verzichtet werden, um untrainierte Schüler nicht schon vor der geforderten Leistung, z.B. Sprint, zu erschöpfen (vgl. dazu auch SÖLL, 1996, 122ff). 


\section{Hauptteil A: Schulung konditioneller Fähigkeiten / Gymnastik}

Dieser Teil dauert fünfzehn Minuten. Das Ziel dieses Teils ist die Förderung der konditionellen Fähigkeiten durch verschiedene gymnastische Übungen. In diesem Teil wird vorausgesetzt, dass die Schüler die Übungen korrekt ausführen und sich anstrengen. Die Übungen dieses Teils sind schwerer in ihrer Durchführung als das Aufwärmen. Deshalb ist bei den Übungen laut MADHKUR (1997, 42f) zu berücksichtigen, dass

- sie alle Körperteile bearbeiten;

- ständig vorkommende Fehler korrigiert werden, um körperliche Schäden zu vermeiden;

- keine starre, sondern eine freie Aufstellung der Schüler erfolgt;

- möglichst alle konditionellen Aspekte (Kraft, Schnelligkeit, Ausdauer, Beweglichkeit und Koordination) berücksichtigt und diese unterschiedlich verknüpft werden;

- verschiedene Kleingeräte (z.B. Stab, Seil, Medizinball) eingesetzt werden,

- sie die Gesundheit der Schüler fördern.

\section{Hauptteil B: Schulung sportartspezifischer Fertigkeiten}

Dieser Teil dauert ca. 20 Minuten und ist Kern des Unterrichts. Hier werden also konkrete Fertigkeiten der im Lehrplan vorgesehen Sportarten vermittelt. In diesem Teil wird die Rolle des Lehrers besonders wichtig. Er soll hier (ähnlich wie auch im ersten Hauptteil) anleiten und vormachen. Laut HUMMUS (1997) sei es bezogen auf letzteres aber besser einen Schüler bestimmte Fertigkeiten vormachen zu lassen. Interessanterweise sollen die Schüler besonders in diesem Teil verschiedene erwünschte Eigenschaften, wie z.B. Führungsqualität, Selbständigkeit oder Selbstvertrauen erwerben (vgl. HUMMUS, 1997, 24f).

\section{Schlussteil (Ausklang)}

Dieser Teil dauert ca. drei Minuten. Das Hauptziel dieses Teils ist die psychische Vorbereitung der Schüler auf den weiteren Unterricht und das Zurückehren des Körpers in den normalen Zustand. In diesem Teil werden die im Unterricht benutzten Geräte und Sachen an ihren Platz gebracht. Die Schüler werden zu den Bädern und Umkleideräumen geschickt, um sich zu waschen und dann ihre Kleidung zu wechseln (vgl. ABD EL KARIM, 1999, 35f). 
Abschließend ist festzustellen, dass die ägyptische Unterrichtsstruktur primär den sportspezifischen Zielen (inkl. Freizeit - Schulsport) und der Gesundheit dient, der Sportlehrer jedoch bei der Vermittlung über die Auswahl von Methoden frei verfügen kann. Vor dem Hintergrund der historischen Entwicklung des ägyptischen Schulsystems (Kapitel 2.4.1) wird jedoch deutlich, welchen Einfluss das „Britische System" heute noch hat. Im Prinzip wird der Sport immer noch stringent ja gar militärisch vermittelt und durchgeführt. Dies ist auch Bestandteil der Lehrerausbildung und so ist es sehr wahrscheinlich, dass der Sportunterricht trainingsorientiert abläuft und die Erziehungsziele Sozialverhalten, psychische Eigenschaften oder Freude an der Bewegung gewissermaßen nebenbei und automatisch erledigt werden. Zumindest wird klar, dass die Umsetzung dieser Erziehungsziele in starkem Maße von den methodischen Maßnahmen der Sportlehrer abhängig sind.

\subsubsection{Methodische Empfehlungen}

In Ägypten gibt es keine einheitlichen Richtlinien für die Auswahl der Erziehungsmethoden, jedoch besteht eine breite Übereinstimmung darüber, dass der Lehrer Kenntnisse über individuelle Unterschiede und Unterschiede von Klassen haben muss (vgl. z.B. ABD EL AZIEM, 1993). Er muss weiterhin Kenntnisse über die Nutzung von Sportplätzen, von Geräten und über spezielle Merkmale der Sportaktivitäten haben. Schließlich muss er die Vorteile und Nachteile der Unterrichtsstruktur kennen. Fehlt dem Lehrer eine der oben genannten Voraussetzungen, wirkt sich dies negativ auf die angewandten Unterrichtsmethoden aus (vgl. LUTFI, 1996, 11f). Davon ausgehend formuliert die ägyptische Sportpädagogik allgemeine methodisch-didaktisch Empfehlungen (vgl. ABD EL-AZIEM, 1993; ABD EL KARIM, 1997, 23f; AHMAD, 1999, 99):

1. Der Sportunterricht soll in einzelne zeitliche Abschnitte unterteilt werden (s.o.).

2. Die Altersphasen und ihre körperlichen wie intellektuellen Besonderheiten müssen bei der Wahl der Lerninhalte berücksichtigt werden.

3. Die Unterschiede in und zwischen den einzelnen Klassen müssen berücksichtigt werden. 
4. Motorische Fertigkeiten sollen nach den pädagogischen Leitlinien „vom Leichten zum Schweren“, „Vom Bekannten zum Unbekannten“ und „vom Langsamem zum Schnellen“ vermittelt werden. Inhalte - v.a. auch neue Inhalte - sollen an den jeweiligen Könnensstand angepasst sein und in individuellem Tempo gelehrt werden.

5. Die Begeisterung am und eine aufgeschlossene Grundhaltung zum Sport muss gefördert werden. Daraufhin ist die Vermittlung der Sportarten zu prüfen.

6. Die Ziele bei der Vermittlung der einzelnen Elemente müssen den Schülern erklärt werden.

7. Bewegungen müssen analysiert und Fehler ausgemerzt werden.

8. Veränderungen (incl. der Lernerfolge) sollen dokumentiert werden.

Im vorliegenden Lehrplan Ägyptens, der Inhalte und Unterrichtsstruktur festlegt, werden keine strengen methodisch-didaktischen Richtlinien festgelegt. Es werden jedoch eine Übungsliste für die Gestaltung des Aufwärmens und der Gymnastik sowie einige Modellstunden bereitgestellt. Während für das Aufwärmen acht Spiele vorgestellt werden, sind die Gymnastikempfehlungen (für Hauptteil A) in Individualübungen und Partnerübungen unterteilt. Die Individualübungen bestehen aus sieben Übungen für den Hals, 16 für die Arme, 34 für den Rumpf, 14 für die Beine, drei Entspannungsübungen / Ausklang und fünf Gleichgewichtsübungen. Als Partnerübungen werden 25 Kräftigungsübungen für die Arme und 81 Übungen für die Kräftigung des Rumpfes genannt. Alle Übungen werden ohne Geräte durchgeführt und sind im Lehrplan mit kurzen Erklärungen und Skizzen verdeutlicht. Folgende Abbildung 4 zeigt eine Seite aus dem Lehrplan für die Gymnastik und Abbildung 5 veranschaulicht danach eine von fünf Empfehlungen für eine Unterrichtseinheit. Man beachte dabei, dass die arabische Schrift von rechts nach links und von oben nach unten läuft. 

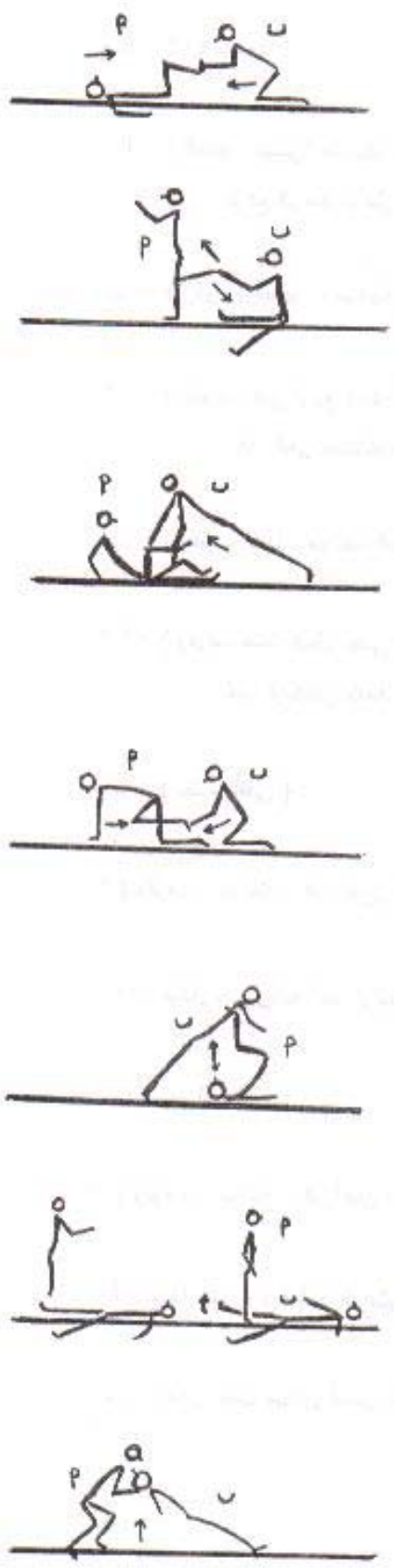

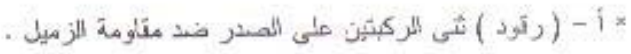

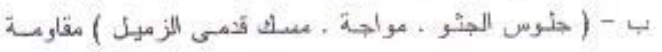

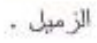

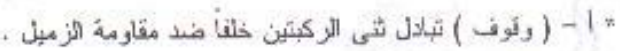

$$
\begin{aligned}
& \text { ب - ( جلوس طويل نثحا , هو أجه ) مقاومة الزميل . }
\end{aligned}
$$

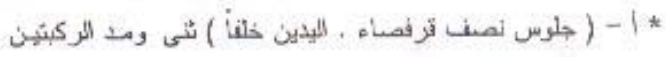

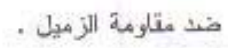

ب - ( انبطأح متل عال مواجة بالارنكز على ركبتين الزميل )

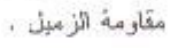

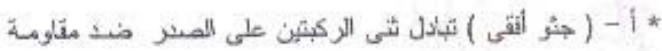

$$
\text { . }
$$

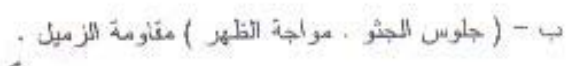

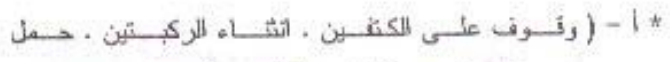$$
\text { المزميل مسر الابطبين بلقدمين ) مد الركيتين . }
$$

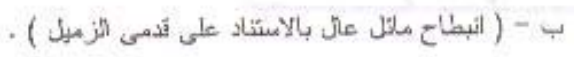

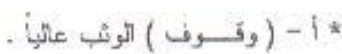

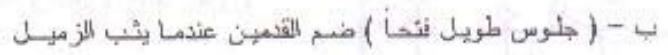

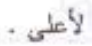

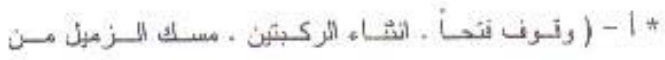

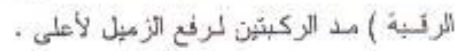

Abbildung 4: Auszug aus dem Lehrplan der Ägyptischen Regierung (Gymnastische Übungen). 


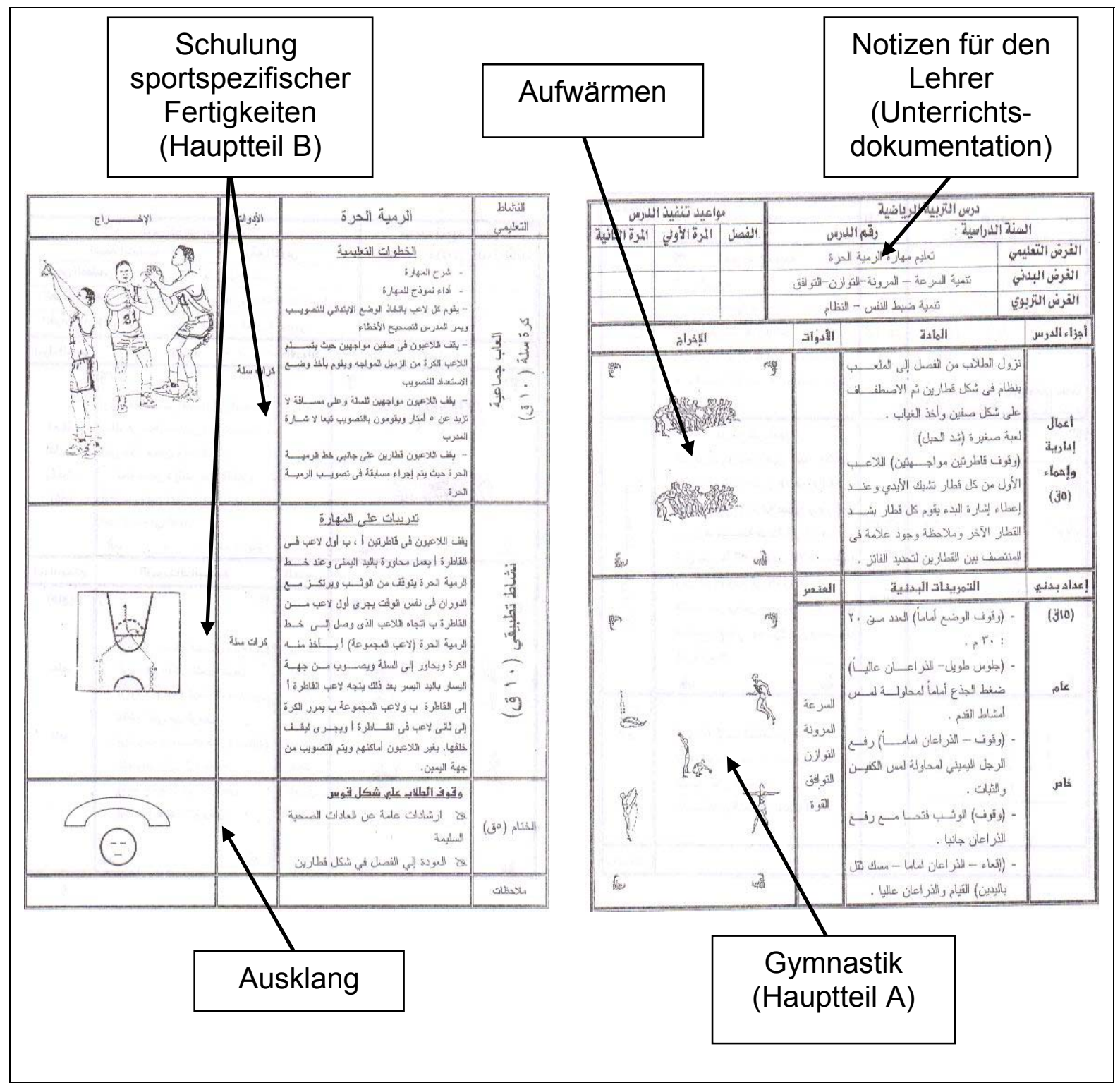

Abbildung 5: Auszug aus dem Lehrplan der Ägyptischen Regierung (Stundenbild).

Im Lehrplan wird in methodischer Hinsicht also lediglich eine Auswahl spezifischer Durchführungsformen genannt. Die stringente Struktur des Unterrichts spiegelt sich auch hier wider. Die Fähigkeiten und Fertigkeiten des Lehrers zur einzelne Ausgestaltung der Unterrichtsteile soll in der (akademischen) Ausbildung geleistet werden. Weitere sich daraus ergebende Aspekte werden daher auch im Rahmen der Sportlehrerausbildung (Kapitel 3.5) ausgeführt. Zuvor sei allerdings noch auf die Bewertung im Sportunterricht und danach auf die Rahmenbedingungen und Hilfsmittel des Sports in der Schule eingegangen. 


\subsubsection{Bewertung durch den Lehrer}

Das Bewerten ist ein wichtiges Unterrichtsverfahren. Man erkennt dadurch, in welchem Umfange die Ziele erreicht wurden und kann als Lehrer die eigene Leistung erkennen. So hilft das Auswerten beim Abschätzen des Erfolges von Unterrichtsverfahren. Daneben zielt die Bewertung auch auf die Feststellung des Niveaus im Vergleich zu anderen. In deren Folge ist die gezielte Arbeit an Stärken und Schwächen (sowohl bei Schülern als auch bei Lehrern) möglich. Durch die Ergebnisse können die für den Lehrplan Verantwortlichen also objektive Entscheidung darüber treffen, ob der Lehrplan abgeändert oder beibehalten werden soll. Deshalb muss das Auswerten parallel zur Durchführung des Lehrplanes geschehen (vgl. El HAMAHMI \& EL CHOULI, 1990, 51f). Die fortgesetzte Leistungskontrolle gibt dem Lehrer nach Ansicht von RIDWAN (1997) laufend Kenntnis darüber, welche Lerninhalte tatsächlich vermittelt worden sind, sodass er seinen Unterricht fortwährend variieren und damit den bestmöglichen Erfolg erzielen kann. Umgekehrt meint allerdings YA'QUUT (1992, 36f), dass mit dem Ende des Sportunterrichts im Verlauf des Schuljahres erst die Auswertung beginnt. Insgesamt geht es jedoch um die Erkenntnis für den Lehrenden, inwieweit die Fertigkeiten von den Schülern erworben worden sind, worüber sich wieder Korrekturmöglichkeiten des eigenen Handelns ergeben. Während EL HAMAHMI \& EL CHOULI und RIDWAN eher die angewandten Methoden in den Blick nehmen, konzentriert sich YA'QUUT eher auf das ganze (Jahres-) Programm.

Weitgehende Unklarheit besteht allerdings in der Anwendung von Bewertungsmethoden. Jedem Lehrer wird die Bewertung zwar grundsätzlich nahe gelegt, jedoch bestehen keine Handreichungen hinsichtlich der Bewertungskriterien. Einzige Empfehlung besteht darin, dass die Bewertung möglichst objektiv sein sollte. Jedoch sollen die Ergebnisse in Sportunterricht und Schulsport dokumentiert werden, wozu sie vorher bewertet sein müssen. Auf mehreren Seiten werden im Lehrplan Testmethoden wie Slalomlauf, Geschicklichkeitsübungen (Gleichgewicht), Zielwurf oder Weitwurf (Messen) beschrieben, ohne jedoch Kriterien oder Ablaufempfehlungen zu nennen. Insofern ist die Bewertungspraxis zu prüfen. 


\subsection{Rahmenbedingungen und Hilfsmittel}

Voraussetzung zum Erlernen und Durchführen des Sports in der Schule ist das Vorhandensein von Sportstätten, Sportgeräten und weiteren Hilfsmitteln. Weil das Fehlen solcher Mittel als Hindernis für die gute Durchführung des Lehrplanes gilt wobei man diesen Lehrplan als quantitativ, qualitativ und strukturell für gut entworfen ansieht -, hat sich der Sportlehrer darum zu kümmern (vgl. DARWISH, EL HAMAHMI \& EL MUHANDES, 1990, 27f; EL GOHARIE, 2002). Teilweise fällt dies aber auch in den Aufgabenbereich des Bezirksleiters (siehe S. 45).

Zunächst ist im Lehrplan das Verhalten bei Unfällen aufgegriffen. Ein Unfallkoffer soll vorhanden sein und vom Sportlehrer gepflegt werden. Weiterhin sind im Lehrplan konkrete Inhalte des Unfallkoffers wie beispielsweise Mercurochrom (zur Wunddesinfektion), Paracetamol (Schmerzmittel) oder Aspirin genannt. Im Falle eines Unfalls muss der Lehrer sofort mit dem Schüler in das nächste Krankenhaus. In leichteren Fällen soll er sofortige Hilfe leisten und einen Arzt kontaktieren. Zur Hilfe werden für typische Zwischenfälle wie Sonnenstich, Nasenbluten oder leichten Schnittverletzungen konkrete Notfallmaßnahmen genannt. Wichtigste Aufgabe des Sportlehrers ist jedoch die Unfallvermeidung, wobei hier Empfehlungen wie Warnhinweise geben, Gerätepflege und Hilfestellungen erfolgen.

Die Rahmenbedingungen des Sporttreibens in der Schule sind die Sportstätten. Für alle Schulformen (Grund-, Mittel- und Oberschulen) gelten die gleichen Richtlinien. Vom Ministerium für Erziehung und Unterricht wird gefordert, dass in einer Schule für die Spielsportarten (Fußball, Handball, Basketball und Volleyball) je regelgerechte Spielfelder vorhanden sind, sowie eine Weitsprunggrube mit $A b$ sprungbrett und ein Wurf- bzW. Stoßkreis (für Kugelstoß und Diskuswurf). Weil der Sportunterricht im Freien stattfindet, werden die Spielfelder auch zum Turnen, zur Leichtathletik und zur Gymnastik genutzt.

Für die Bezirksleiter der einzelnen Schulen, die für übergeordnete Inventarisierung der Sportstätten und Unterrichtsmaterialien verantwortlich sind, werden im Lehrplan konkrete Checklisten veröffentlicht. Diese sind wiederum für alle Schulformen identisch und beziehen sich zunächst auf die Anzahl von Bällen: Für zehn Schüler soll insgesamt ein Lederball zur Verfügung stehen. Für die Bälle der vier 
Spielsportarten richtet sich die Anzahl nach der Schulgröße. Demnach teilen sich möglichst 100-500 Schüle fünf bis acht Bälle, 501-1000 Schüler acht bis zwölf Bälle und 1001-1500 Schüler zwölf bis fünfzehn Bälle. Dies bedeutet vor dem Hintergrund der Schulgröße (siehe Kapitel 2.4.2) und bei den vier Spielsportarten u.U. nur ein Ball pro Sportart und Schule - und bei ebenfalls einem Ball/Klasse (ca. 40 Schüler) sollen die Sportarten dann vermittelt werden. Daneben ist im Lehrplan eine Checkliste ohne Mengenempfehlungen abgedruckt, die folgende Sportgeräte und Hilfsmittel umfasst:

- Sandgrube, Hochsprungstange und -matte, Diskus, Kugeln, Hürden, Speere

- Turnmatten, Kasten, Reck, Turnbänke, Springseile, Keulen, Reifen, Stäbe, Medizinbälle,

- Fußballtore, Handballtore, Basketballkörbe, Volleyballnetz, Tischtennisplatte, viele verschiedenen Bälle, Fußball, Basketball, Volleyball und Handball, Ersatzbälle, Gummiball,

- Stoppuhr, Luftpumpe, Megafon (Flüstertüte) für Turniere, lange Maßbänder und Fähnchen.

Da die Beobachtungen des Autors jedoch Zweifel am umfangreichen Vorhandensein solcher Hilfsmittel aufkommen ließen, ist auch die Kategorie Rahmenbedingungen/Hilfsmittel empirisch zu prüfen.

\subsection{Sportlehrerausbildungen}

Bei steigender Arbeitslosigkeit in Ägypten (und bei nicht vorhandenem Arbeitslosengeld) ist der Sportlehrerberuf für viele attraktiv, weil viele Arbeitsstellen frei sind und ein Arbeitsplatz nach der Ausbildung praktisch gesichert ist. Allerdings ist der Verdienst mit etwa 20,- $€$ nicht sehr hoch, denn er reicht gerade für die Grundversorgung einer dreiköpfigen Familie. Trotzdem ist die Zahl der Studienanfänger des Faches Sport relativ hoch und die Versorgung der Schulen mit Lehrkräften scheint in näherer Zukunft gesichert.

Der Sportlehrerberuf ist nun mit der Vermittlung der Erziehungsziele, der Durchführung des Unterrichts und der weiteren Aufgaben befasst. Er erfordert eine ge- 
zielte Ausbildung, in der die Lehramtsanwärter für die geforderten Aufgaben verantwortungsvoll vorbereitet werden. Mit der universitären Ausbildung der Sportlehrer und -lehrerinnen soll dies in ausreichend quantitativer und v.a. qualitativer Form gesichert werden. In den sieben Sportinstituten Ägyptens werden in erster Linie Kenntnisse in Unterrichtsplanung, Unterrichtsinhalten, Unterrichtsmethoden und Besonderheiten ihrer Anwendung vermittelt (vgl. EL GOHARIE, 2002).

Die Studenten werden für acht Semester in ein Sportinstitut aufgenommen, wenn sie das Abitur und die Aufnahmeprüfung bestanden haben. Die Prüfung besteht in erster Linie aus praktischen Tests. In einem Gespräch (Interview) mit dem einzelnen Kandidaten wird jedoch zuvor seine sprachliche Ausdrucksfähigkeit geprüft, außerdem werden Größe, Gewicht, Puls und Blutdruck gemessen und jeder Kandidat auf Körperhaltungsschäden untersucht. Erst nach dieser Eignungsprüfung wird man zur praktischen Prüfung zugelassen. Ein erster Teil der praktischen Prüfung orientiert sich an den konditionellen Eigenschaften Kraft, Schnellkraft, Ausdauer und Schnelligkeit. In jedem Test (100m-Lauf, 800m-Lauf, Klimmzüge, Standweitsprung, Hockstrecksprünge) ist eine bestimmte Punktzahl zu erlangen. In einem zweiten Teil wird der Kandidat in einer frei wählbaren Sportart geprüft. Dabei muss er die grundlegenden Techniken der Sportart vorführen. Die Summe aller Punkte entscheidet dann darüber, ob der Kandidat bestanden hat.

Im Rahmen des Studiums können zunächst zwei grundlegende Bildungsbereiche voneinander abgegrenzt werden: Einerseits soll mit der Ausbildung (meist implizit) die Allgemeinbildung und Persönlichkeitsentwicklung gefördert werden (vgl. ZAHRAN, 1996, 76f). Andererseits erfolgt eine explizite fachspezifische Ausbildung, die sich wiederum in zwei Bereiche unterteilen lässt, nämlich die theoretische und die fachpraktische Ausbildung. Im Folgenden seien die drei Bildungsbereiche dargestellt (vgl. dazu die umfassenden Arbeiten von ABD EL AZIEM, 1993; EL GOHARIE, 2002). 


\subsubsection{Förderung der Persönlichkeit}

Eine gefestigte Persönlichkeit ist einer der wichtigsten Faktoren für den Erfolg des Sportlehrers in seinen Aufgaben. Obwohl es schwer ist, Merkmale einer „guten“ Lehrerpersönlichkeit zu definieren, sollte der ägyptische Sportstudent folgende förderlichen Eigenschaften erwerben (vgl. auch ÄGYPTISCHES MINISTERIUM FÜR ERZIEHUNG UND UNTERRICHT, 1998):

Er soll

- seinen Beruf mögen und selbst an seine Botschaften glauben;

- $\quad$ seine Emotionen im Umgang mit anderen beherrschen können, Selbstvertrauen haben und motivieren können;

- $\quad$ eine gepflegte äußerliche Erscheinung haben, pünktlich sein und mit Kollegen ein gutes Verhältnis pflegen;

- Kenntnisse zu psychischen, sozialen und kulturellen Aspekten der Jugend haben;

- $\quad$ nicht zu streng sein;

- selbständig Probleme lösen können;

- Fähigkeiten im Bereich von Organisation und Verwaltung haben;

- sportlich geschickt und körperlich gesund sein.

Darüber hinaus sollte er eine gute Allgemeinbildung aufweisen. Dazu gehören Kenntnisse zu allgemeinen gesellschaftlichen Gegebenheiten und Umgangsformen des öffentlichen Lebens (z.B. religiöse, erzieherische, gesundheitliche oder wirtschaftliche Aspekte), Wissen um lokale und internationale gesellschaftliche Probleme und Positionen, Kenntnisse zur Mediennutzung und schließlich die Bereitschaft zur Weiterentwicklung durch aktive Teilnahme am öffentlichen Leben.

Die an der Universität eingesetzten Mittel für eine entsprechende Förderung werden hier stichpunktartig genannt. Sie sind zum größten Teil in die fachspezifische Ausbildung integriert (vgl. KAMEL, SHALTUT \& CHAFAGA, 2002, 13f): 
1. Durch die Nachahmung der vorbildlichen Hochschullehrer sollen die Lehramtsanwärter selbst zum Vorbild werden ${ }^{16}$.

2. Die Kandidaten sollen dazu ermutigt werden, eine bewusste Verbindung zwischen dem fachspezifischen Wissen und ihrer eigenen Persönlichkeit herzustellen.

3. Die sozialen, kulturellen und studentischen Aktivitäten sollen eine eigenständige Auseinandersetzung mit dem zukünftigen Beruf und die Beziehung untereinander fördern.

4. Durch Prämien und Ehrenurkunden sowie durch Ermutigungen der Dozenten während der Veranstaltungen sollen Anreize für den eigenen, zu erbringenden, und Belohnungen für den bereits erbrachten Aufwand geschaffen werden.

\subsubsection{Theoretische Ausbildung}

Am Anfang der theoretischen Ausbildung steht die Vermittlung von grundlegenden übergreifenden Fertigkeiten wie Arabisch, Englisch sowie der Umgang mit einem PC und am Ende werden wissenschaftliche Methoden wie Versuchsplanung, Datenerhebung, Datenauswertung (Statistik) sowie deren Darstellung behandelt. Hauptsächlich sollen die Sportstudenten bei der theoretischen Ausbildung jedoch unterschiedliche Teilgebiete der Sportwissenschaft kennen lernen. Schwerpunktmäßig werden Sportpädagogik, Sportpsychologie/Sportsoziologie, Sportmedizin (funktionelle Anatomie/Physiologie/Erste Hilfe), Sportgeschichte, Biomechanik und Trainingslehre behandelt. In erster Linie geht es darum, wie dieses Wissen auf die Unterrichtsgestaltung und auf das Training/die Organisation im Schulsport zu beziehen ist und so nimmt die Sportdidaktik bzw. die Methodenvermittlung bei der Sportlehrerausbildung eine zentrale Stellung ein, „weil der Erfolg des Erziehungsverfahrens als Ganzes davon abhängig ist, welche Methoden die Verantwortliche im Einzelnen anwenden“ (ABD EL RAHMAN, 1999, 69). Zusammenfassend erge-

\footnotetext{
${ }^{16}$ Wenn die ägyptische Sportpädagogik Aussagen zu Lehrplänen oder Lehrmethoden trifft, wird meist auch die Lehrerausbildung thematisiert. Dabei werden Empfehlungen für Hochschullehrer getätigt, die den Empfehlungen für Lehrern sehr ähneln. EL GOHARIE (2002) hebt beispielsweise Rückmeldungen (Feedback) heraus, empfiehlt jedoch auch darüber hinausgehend, dass akademische Lehrer Bücher schreiben und Poster oder gar Filme bei der Ausbildung der Studenten verwenden sollen.
} 
ben sich im Hinblick auf die Sportdidaktik folgende Studienschwerpunkte (vgl. ABD EL AZIEM, 1993; AHMAD 1996, 35f; BAHA'ALDIN, 1997, 55f; EL SHAHED, 1997, 17f) ${ }^{17}$ :

1. Kindliche Entwicklung mit der Betonung von Wachstumsprozessen und von motorischer Entwicklung

2. Funktionen und Funktionsweise menschlicher Bewegung

3. Kenntnis der Regelwerke und Schiedsrichterkenntnisse

4. Bestehende Lehrpläne und Möglichkeiten der Umsetzung:

a) Vorbereitung und Planung des Sportunterrichts

b) Erziehungsziele und Diskussion/Reflexion des Erziehungsprozesses

c) Unterrichtsmethoden und Strategien der sportpraktischen Vermittlung

d) Beobachtung und Beurteilung von Bewegungen

e) Dokumentation des Unterrichts

Im Kern der Lehrerausbildung steht der 4. Punkt, also die Umsetzung der Lehrpläne. Alle drei „Perspektiven“ der ägyptischen Sportpädagogik (vgl. ABD EL AZIEM, 1993), nämlich Kenntnis des Lehrplans, Bedürfnisse und Neigungen der Lehrer und Schüler sowie konkrete Hilfestellungen, sollen dabei Berücksichtigung finden. Betont wird jedoch letzter Aspekt und so ist der Großteil der Ausbildung mit der konkreten Gestaltung des Sportunterrichts (bzw. des Schulsports) befasst. Folglich findet auch eine enge Verschränkung von theoretischer und fachpraktischer Ausbildung statt. So werden in der fachpraktischen Ausbildung (s.u.) theoretische Inhalte auf die einzelnen Sportarten bezogen.

Insgesamt sollen die Studenten sich dabei selbstständig neues Praxiswissen erarbeiten und vielfältige Möglichkeiten der Umsetzung kennen lernen. Dazu gehören auch die treffende Anwendung von Lehrmethoden für große Klassen und die Berücksichtigung der Wetterbedingungen (vgl. auch ABD EL AZIEM, 1993; EL GOHARIE, 2002; EL SHAHED, 1997). In Bezug auf die Methodik werden konkret folgende Lehrinhalte gefordert und vermittelt:

\footnotetext{
${ }^{17}$ Die Studenten sollen dazu verschiedene Schriften kennen. Neben den Veranstaltungen soll also innerhalb des Studiums selbständig Wissen aus Fachbüchern erschlossen werden, die den Studenten zur Verfügung gestellt werden.
} 
- Schülergerechte Anweisungen und Erklärungen im Unterricht

- Vermittlung motorischer Fertigkeiten nach den pädagogischen Leitlinien „vom Leichten zum Schweren“, „vom Bekannten zum Unbekannten“ und „vom Langsamem zum Schnellen“. Dabei sollen die Inhalte an den jeweiligen Könnensstand angepasst werden können. Für das Unterrichten der sportspezifischen Fertigkeiten werden im Großen und Ganzen drei Methoden vermittelt:

\section{Ganzheitsmethode}

Komplexe Bewegungsabläufe sollen zuerst vorgemacht und dann nachgemacht werden. Diese Methode soll im Unterricht wegen der großen Schülerzahl und der kurzen Unterrichtszeit bevorzugt werden. Als weitere Argumente für die Ganzheitsmethode nennt ABD EL AZIEM, dass sie auch auf niedrigem Niveau durchgeführt werden kann, ein Gesamteindruck der Sportart vermittelt wird und sie schließlich abwechslungsreich und altersgemäß angewandt werden kann.

\section{Teilmethode}

Hier werden einzelne Teile von komplexen Fertigkeiten vermitteln. Dazu ist es notwendig, dass die Lehrer selbst über ein gehobenes Fertigkeitsniveau verfügen und die Beziehungen der einzelnen Teilbewegungen zur Gesamtbewegung bekannt sind. Besonders geeignet sei diese Methode für ein höheres Niveau der Fertigkeitsvermittlung (z.B. in der Oberschule), für den Schulsport und für kleine Klassen.

\section{Ganz-Teil-Methode}

Diese Methode setzt die zuvor genannten zusammen. Zuerst soll möglichst die Ganzheitsmethode angewandt und erst bei Problemen auf die Teilmethode zurückgegriffen werden. Hier wird die Teilmethode also zur Problemlösung eingesetzt. Neuerdings wird die Verknüpfung der Methoden allerdings differenzierter gelehrt (vgl. EL GOHARIE, 2002), nämlich nach Ganz-Teil-Methode und zusätzlich nach Teil-Ganz-Methode, bei der die einzelnen Bewegungsteile sukzessive bis zur Gesamtbewegung zusammengesetzt werden.

- Spiel- und Übungsformen zum Aufwärmen

- Schaffen eines Bewusstsein für die Notwendigkeit von Bewertungsmethoden. Hier wird gelehrt, wie die Bewertungsmethoden zur gezielten Entwicklungsför- 
derung eingesetzt werden können, wie gemessen und dokumentiert wird. In den einzelnen Sportarten sollen die Studenten gar eigene Bewertungsmethoden entwickeln, vorstellen und diskutieren.

- Erkennen von Problemen bei der Vermittlung, deren Diskussion und selbständige Lösung. In diesem Rahmen sollen die Studenten auch verschiedene Führungsstile kennen lernen.

\subsubsection{Fachpraktische Ausbildung}

In der fachpraktischen Ausbildung lernen die Studenten zunächst selbst die wichtigsten Sportarten, um sie daraufhin selbst auf gehobenem Niveau vermitteln zu können. Einerseits sind die Mannschaftssportarten Fußball, Basketball, Volleyball, Handball und Hockey und andererseits die Individualsportarten Leichtathletik, Turnen, Schwimmen, Tischtennis, Boxen, Ringen und Fechten zu nennen. Daneben werden Gymnastik und Kleine Spiele gelehrt.

Durch diese praktische Vermittlung bekommen die Studenten auch ein entsprechendes Bild davon, wie sie selbst die Sportarten in den Schulen lehren können. Dabei geht es in erster Linie um das Wie (s.o.). Im 3. und 4. Semester erfolgen zusätzlich schon erste kurze Lehrversuche (etwa 15 Min.) mit den Kommilitonen.

Die eigene Erfahrung mit dem Unterrichten wird dann weitergehend in die Ausbildungsstruktur integriert, denn es werden Praktika gefordert. Im dritten Ausbildungsjahr besuchen die Studenten wöchentlich eine Mittelschule und im vierten Ausbildungsjahr wöchentlich eine Oberschule, wo sie unterrichten. In der vorlesungsfreien Zeit muss zwischen dem 5. und 8. Semester weiterhin ein dreiwöchiges Praktikum in einer Schulform absolviert werden. Hier sollen die Studenten ihre Kenntnisse erproben, Selbstvertrauen gewinnen, ein Vorbild für die Schüler werden und sich im Sozialsystem Schule zurechtfinden, wozu auch die Beziehungspflege zu den Eltern gehört. Abschließend werden die Studenten von den Schullehrern beurteilt und sollen selbst einen Bericht schreiben. 


\subsection{Fazit}

In diesem Kapitel wird der Sportunterricht anhand einiger Strukturmerkmale charakterisiert. $\mathrm{Er}$ ist maßgeblich von den ausformulierten Richtlinien des ÄGYPTISCHEN MINISTERIUMS FÜR ERZIEHUNG UND UNTERRICHT bestimmt, die in erster Linie für das Unterrichten durch die Sportlehrer gedacht sind. Zum Sportlehrer selbst (als direkter Vermittler dieser Richtlinien) werden einige wünschenswerte Persönlichkeitseigenschaften und vor allem seine Aufgaben formuliert. Neben der Vermittlung bestimmter Inhalte nach bestimmten Methoden unter bestimmten Rahmenbedingungen (s.u.) scheint hier wesentlich, dass nicht nur der Sportunterricht, sondern vielmehr auch der Schulsport durchgeführt werden soll. Letzterer dient zu Showeinlagen bei Feierlichkeiten oder einem leistungssportlichen Trainieren. Aufgrund des Gewichtes des Schulsports (im Hinblick auf die Sportstruktur Ägyptens und die Reputation von Lehrern) ist zu vermuten, dass dies auch deutliche Auswirkungen auf die Gestaltung des Sportunterrichts hat. Mit inm können und sollen Grundlagen für den Schulsport gelegt werden. Vergleichbar ist die große Zielrichtung des ägyptischen Sportunterrichts mit einer frühen Phase der deutschen Leibeserziehung und Sportpädagogik, zu der GRÖBING (1997, 19f) bemerkt: „In der Begründung als Unterrichtsfach tritt das pädagogische Argument zurück (...) - aus der Erziehung durch Sport wird die Erziehung zum Sport." Wesentliche Ursachen für heutige Unterschiede liegen in der verschiedenartigen Kultur, insbesondere aber der differenten Struktur des Sportsystems (siehe Kapitel 3.2.7).

Die Persönlichkeitseigenschaften der Sportlehrer lassen sich nun als erste konkrete Voraussetzungen für das Unterrichten heranziehen. Allerdings sind die thematisierten Eigenschaften kaum einer empirischen Prüfung zugänglich. Aus diesem Grunde erscheint es zweckmäßig, diese Voraussetzungen einerseits auf die Einstellung zum Sportunterricht einzuengen, andererseits aber auch zu präzisieren. So wird also hier davon ausgegangen, dass die Einstellung eine wesentliche Disposition zum Lehrerhandeln darstellt, die sich in verschiedene handlungsspezifische Meinungen bzw. Überzeugungen zu konkreten Merkmale des Sportunterrichts (hier: Erziehungsziele, Inhalte, Methoden, Rahmenbedingungen und Hilfsmittel) zerlegen lässt (vgl. dazu beispielsweise AJZEN 1991; 2001). 
Die Analyse der Einstellung bzw. der Meinungen der Lehrer zum Sportunterricht eröffnet dann Aussagen zu mindestens drei Aspekten. Erstens können die personellen Handlungsvoraussetzungen zur Umsetzung der Richtlinien als günstig oder ungünstig beurteilt werden - beispielsweise im Hinblick auf die Akzeptanz von Erziehungszielen oder methodischen Empfehlungen. Zweitens können Aussagen über die Qualität der Ausbildung getroffen werden und drittens sollten sich Schlussfolgerungen darüber ziehen lassen, inwieweit die gewünschten Aspekte des Lehrplans tatsächlich in die Praxis der ägyptischen Schule passen.

Daraufhin ist aber auch die Wirklichkeit des ägyptischen Sportunterrichts, d.h. die tatsächliche Umsetzung der Richtlinien, zu prüfen. Auch hier stellen die Sportlehrer einen wesentlichen Ansatzpunkt dar, denn sie sind nicht nur für die Umsetzung der Lehrpläne zuständig, sondern beobachten die Schüler bei der Sportausübung, kennen die auftretenden Schwierigkeiten, wissen welche Sportarten und Übungen den Neigungen der Schüler entgegenkommen und welche aufgrund der vorhandenen Möglichkeiten umsetzbar sind - kurzum: sie kennen und adaptieren den praktischen Alltag des Sportunterrichts. Im Sinne einer ersten Exploration kann dies dann mit den Lehrermeinungen konfrontiert werden, was unter Berücksichtigung der strukturellen Bedingungen insgesamt Aussagen zur Optimierung des ägyptischen Sportunterrichts und seiner Richtlinien erlauben sollte. Zusammenfassend richtet sich die nachfolgende empirische Analyse (Befragung der Lehrer) damit auf zwei zentrale Fragen:

1. Wie sollte der Sportunterricht in der Arabischen Republik Ägypten aus Sicht der Sportlehrer sein? und

2. Wie läuft er tatsächlich in der Praxis $a b$ ?

Diese zentralen Fragen sind auf die dargestellte Struktur des Sportunterrichts zu beziehen, deren Kategorien sich wiederum unterschiedlich zerlegen lassen. Zunächst betrifft dies die Erziehungsziele. Die einzelnen Analyseeinheiten oder Unterkategorien wurden bereits am Ende von Kapitel 3.2.7 (S. 62 unten) zusammengefasst und seien hier nicht nochmals wiederholt.

Ebenso sind Inhalte und Methoden zu analysieren, die eng miteinander verknüpft sind (vgl. auch BADIR, 1998). Mit der Jahresplanung sind die Inhalte der Sportarten und mit der rigiden Strukturierung der einzelnen Unterrichtseinheit die einzel- 
nen Abläufe seit Jahren festgelegt und so eignet sie sich wenig als Kriterien für eine Analyse. Da die unterschiedlichen Teilaspekte der Inhalte wie technische und taktische Fertigkeiten der Sportarten einerseits, sowie die konditionellen Fähigkeiten im Rahmen der Gymnastik andererseits weiten Spielraum lassen, sollen diese als inhaltliche Schwerpunkte untersucht werden. Damit ist also keine Jahresplanung gemeint, sondern die konkrete schwerpunktmäßige Ausgestaltung der Unterrichtseinheiten nach Zielbereichen. Dies könnte beispielsweise Aufschluss darüber geben, ob unverhältnismäßig wenige koordinative Fähigkeiten gegenüber Kraftfähigkeiten geschult oder ob mehr technisch-taktische Finessen gegenüber grundlegenden motorischen Mustern vermittelt werden.

Daneben lässt auch die Strukturierung der Unterrichtseinheiten immer noch großen Spielraum für unterschiedliche Unterrichtsmethoden. Festzustellen ist jedoch, dass die Methodenfrage in der ägyptischen Sportpädagogik bisher wenig systematisch aufgearbeitet wurde, was sich auch in den Lehrplänen des ÄGYPTISCHEN MINISTERIUMS FÜR ERZIEHUNG UND UNTERRICHT niederschlägt. Zwar kann vermutet werden, dass der Sportunterricht trainingsorientiert abläuft, jedoch ist noch nichts über das Wie bekannt ${ }^{18}$. Dies macht deutlich, dass die Methodenfrage nicht isoliert, sondern verbunden mit anderen Einflussgrößen und Aufgaben des Unterrichts zu betrachten ist (s.o.; vgl. MEYER, 1989, 35). Mit MEYER wird daher zunächst davon ausgegangen, dass die Frage nach den Vorund Nachteilen einzelner methodischer Maßnahmen im praktischen Unterricht in den Hintergrund tritt und Gesichtspunkte im Vordergrund stehen, ob die Aufgabenstellung von den Schülern verstanden wurde, die Übung begriffen oder ob der Zeitrahmen vergrößert werden muss. Insofern sind zunächst Aspekte der allgemeinen Methodik in den Blick zu nehmen. In Anlehnung an die Empfehlungen der ägyptischen Sportdidaktik (siehe Kapitel 3.3.3 und 3.5.2) scheinen hier folgende Kategorien fruchtbar: Vormachen der Übungen, schülergerechte Anweisungen, Rückmeldungen (richtig/falsch), Prüfungen zur Vermittlung von Erfolgserlebnissen, Prüfungsvorbereitungen, selbständige Problemlösungen, Wettkampfdurchführungen und sportgerechte Kleidung. Bei der Analyse einer solchen allgemeinen Me-

\footnotetext{
${ }^{18}$ Mit Blick auf die Erziehungsziele konnte gezeigt werden, dass die Unterrichtsstruktur sich explizit auf sportspezifische Ziele und Gesundheit richtet und nur implizit auf Sozialverhalten, psychische Eigenschaften oder Freude an der Bewegung.
} 
thodik kann die Analyse jedoch nicht stehen bleiben und so gewinnen ebenso Aspekte an Bedeutung, die der sportspezifischen Methodik zuzuordnen sind. Dabei steht die Frage im Vordergrund, inwieweit die „großen Methoden“ der Sportdidaktik (Ganzheitsmethode, Teilmethode und Ganz-Teilmethode) bei der Vermittlung sportartspezifischer Fertigkeiten Anwendung finden und ob sich bei den verschiedenen Sportarten Unterschiede ergeben. Daneben scheint noch interessant, inwiefern die Sportlehrer bei den großen Klassen noch individuell auf die Schüler eingehen können und wollen. Schließlich wurde in Kapitel 3.4 und 3.5 auf die Notwendigkeit von Bewertungsmethoden hingewiesen. Aufgrund der fehlenden Sportnoten im Zeugnis ist auch hier fraglich, inwieweit sie Anwendung finden. Um hierüber Aufschluss zu bekommen, wird danach gefragt, ob in der Praxis bewertet wird und welche Kriterien für eine solche Bewertung herangezogen werden sollten. Insgesamt wird die Methodenfrage also auf vier Unterkategorien bezogen, nämlich auf allgemeine Methoden, auf sportspezifische Methoden, auf Bewertungsmethoden und auf inhaltliche Schwerpunkte.

Schließlich sind die Rahmenbedingungen und Hilfsmittel des Sportunterrichts anhand der Lehrerbefragung zu erheben. Darüber können die Voraussetzungen dafür geprüft werden, ob der Sportunterricht die an inn gestellten Erwartungen grundsätzlich erreichen kann. Ist beispielsweise kein Basketballkorb vorhanden, wird die Vermittlung von Basketball praktisch unmöglich. Dies bedeutet, dass die Checklisten der Bezirksleiter noch keinen Aufschluss über das tatsächliche Vorliegen der Rahmenbedingungen und Hilfsmittel zulassen. Für die Untersuchung sollen nun allerdings nicht alle im Lehrplan genannten Hilfsmittel herangezogen werden, sondern nur solche, die der Autor als Voraussetzung zu einer abwechslungsreichen Umsetzung der Richtlinien betrachtet. 


\section{Empirische Untersuchung}

Dieses Kapitel beschreibt nun nochmals zusammenfassend die Methodik der Untersuchung, die zur Beantwortung der am Ende des letzten Kapitels aufgeworfenen Fragen sinnvoll erscheint. Insgesamt sollen mit ihr folgende übergreifende Fragen geklärt werden:

- Werden die übergreifenden Erziehungsziele von den Sportlehrern akzeptiert und im Unterricht berücksichtigt?

- Was wollen die Sportlehrer inhaltlich und methodisch in ihrem Unterricht unternehmen und was machen sie tatsächlich?

- Welche Lernvoraussetzungen sind gegeben, welche sind möglichst noch zu schaffen?

Die Beschreibung der bereits durchgeführten Untersuchung folgt nun dem allgemeinen Schema von BORTZ (1999): Untersuchungsplanung (hier: Fragebogenkonstruktion), Untersuchungsdurchführung (hier: Auswahl der Stichprobe, Aspekte der Datenerhebung) und Auswertung (hier: Datenverarbeitung).

\subsection{Aufbau des Fragebogens}

Der Entwurf des Fragebogens ist die Festlegung der zentralen Kategorien, um die sich die Fragestellungen drehen (siehe dazu auch Kapitel 3.6). Sportlehrer verfügen über Informationen zum Sportunterricht, die sie diesbezüglich als Experten qualifizieren. Neben der schriftlichen Erhebung personaler Merkmale wie Geschlecht, Schulform und Alter ${ }^{19}$ sollten die Sportlehrer also zu folgenden drei Kategorien befragt werden:

\footnotetext{
${ }^{19}$ Aufgrund der in Ägypten fest vorgegebenen Ausbildungsstruktur wurde auf die Erhebung der Berufserfahrung verzichtet. Es wird angenommen, dass das Alter auch die Jahre der Berufserfahrung widerspiegelt.
} 
- Erziehungsziele des Sportunterrichts

- Methodisches Vorgehen im Sportunterricht

- Rahmenbedingungen und Hilfsmittel des Sportunterrichts

Für die Ausgestaltung der Fragen zu den Erziehungszielen wurde Kapitel 3.2 herangezogen. Die vom Ministerium für Erziehung und Unterricht vorgegeben Zielbereiche finden sich daher auch als Unterkategorien wieder (siehe dazu den Überblick zum Fragebogen, Tab. 8). Daneben wurde das methodische Vorgehen beleuchtet, das im Lehrplan weitgehend unberücksichtigt bleibt. Hier erscheinen als Unterkategorien das allgemeine methodische Vorgehen, das sportspezifische methodische Vorgehen, die inhaltlichen Schwerpunkte und die Bewertungsmethoden interessant (siehe Kapitel 3.3). Schließlich wurden auch die Rahmenbedingungen und Hilfsmittel empirisch erhoben (3.4).

Die weitere Konstruktion der Fragen berücksichtigte dann zwei Aspekte: Zum Einen wurde nach den Meinungen der Sportlehrer gefragt und zum Anderen nach der tatsächlichen Umsetzung dieser Inhalte in der Praxis. Hierüber sollte festgestellt werden, inwiefern Bedingungen für zukünftige Veränderungen gegeben sind und was tatsächlich in der Praxis des ägyptischen Sportunterrichts geschieht; es soll herausgefunden werden, wie sich die drei o.g. Kategorien im Denken der Sportlehrer und in der Praxis darstellen.

Schließlich ist zu bemerken, dass am häufigsten fünfstufige Likert-Skalen als Messskalen verwendet wurden. Wo nötig und für einen ersten Überblick hilfreich, wurde auch auf einfache Nominalskalen zurückgegriffen, beispielsweise zum Ermitteln von Unterschieden im Unterrichten einzelner Sportarten. Zur Validierung einzelner Aussagen wurde zusätzlich nach der Anwendung einzelner individueller Methoden gefragt ${ }^{20}$.

${ }^{20}$ Dem Anhang ist der gesamte Fragebogen beigefügt. 


\begin{tabular}{|c|c|c|c|}
\hline \multirow[b]{2}{*}{ Kategorien } & \multirow[b]{2}{*}{ Unterkategorien } & \multicolumn{2}{|c|}{ Items } \\
\hline & & $\begin{array}{l}\text { Meinungen } \\
\text { der Sport- } \\
\text { lehrer }\end{array}$ & $\begin{array}{l}\text { Praxis des Sport- } \\
\text { unterrichts }\end{array}$ \\
\hline \multirow{6}{*}{ Erziehungsziele } & Freizeit & $1-3$ & $47-48$ \\
\hline & Gesundheit & $4-7$ & $49-53$ \\
\hline & Sozialverhalten & $8-12$ & $54-58$ \\
\hline & Psychische Eigenschaften & $13-16$ & $59-63$ \\
\hline & $\begin{array}{c}\text { Sportspezifische } \\
\text { Kenntnisse }\end{array}$ & $17-20$ & $64-67$ \\
\hline & Freude/Spaß/Begeisterung & $21-24$ & $68-74$ \\
\hline \multirow{4}{*}{$\begin{array}{l}\text { Methodisches } \\
\text { Vorgehen }\end{array}$} & Allgemeine Methodik & $25-33$ & $75-81$ \\
\hline & $\begin{array}{l}\text { Methodik bei der sportart- } \\
\text { spezifischen Fertigkeits- } \\
\text { vermittlung }\end{array}$ & $34-36$ & $82-83$ \\
\hline & Inhaltliche Schwerpunkte & $37-43$ & $84-85$ \\
\hline & Bewertungsmethoden & $44-45$ & $86-87$ \\
\hline $\begin{array}{c}\text { Rahmenbedingungen } \\
\text { und Hilfsmittel }\end{array}$ & - & 46 & $88-89$ \\
\hline
\end{tabular}

Tabelle 8: Aufbau des Fragebogens.

\subsection{Stichprobenauswahl und Datenerhebung}

Um die Ziele der Untersuchung zu erreichen, mussten bestimmte Voraussetzung bei der Auswahl der Untersuchungsstichprobe berücksichtigen werden. Die ausgewählten Lehrer und Lehrerinnen sollten eine qualifizierte, akademische Ausbildung durchlaufen haben und die Lehrmittel und die Lehrmethoden kennen. Dies wurde durch deren Ausbildung zunächst vereinfachend als Gegeben vorausgesetzt.

Weiterhin sollten verschiedene Schulformen in den Blick genommen und Unterschiede aufgedeckt werden. Um die Untersuchung nicht zu weit auszudehnen fiel eine Entscheidung auf Mittel- und Oberschulen.

Der Bezirk El Dakahlia (in dessen Hauptstadt El Mansoura auch das Heimatinstitut des Autors liegt) wurde auch ausgewählt, weil er mitten im Nil-Delta liegt, was ihn repräsentativ für eine große Anzahl von Bezirken macht. Tabelle 9 gibt zunächst einen Überblick zu Schulenanzahl und entsprechenden Sportlehrkräften im Jahre 2002. Daran wird ersichtlich, dass die Verhältnisse von Sportlehrern und Schulen erheblich variieren können (vgl. auch Tabelle 5, S. 42). Während in diesem Bezirk knapp eine Sportlehrerin für eine Mädchen-Mittelschule zur Verfügung steht, un- 
terrichten bei sonst durchschnittlichen Werten gar vier Sportlehrer pro JungenOberschule.

\begin{tabular}{|r|c|c|c|}
\hline & Mittelschule & Oberschule & Gesamt \\
\hline \hline Jungenschulen & 169 & 25 & 194 \\
\hline Mädchenschulen & 180 & 24 & 204 \\
\hline Sportlehrer & 417 & 99 & 516 \\
\hline Sportlehrerinnen & 167 & 50 & 217 \\
\hline \hline \multicolumn{2}{|r|}{ Gesamtzahl der Sportlehrkräfte } & $\mathbf{7 3 3}$ \\
\hline
\end{tabular}

Tabelle 9: Zahl der Schulen und Sportlehrkräfte im Bezirk El Dakahlia (Quelle: ZENTRALES AMT FÜR ÖFFENTLICHE ERHEBUNG UND STATISTIK, 2002).

Die Stichprobenauswahl für die Untersuchung erfolgte zufällig. Verschiedene Schulen wurden direkt besucht. Dazu wurde das persönliche Stipendium des Autors unterbrochen und eine Reise nach El Mansoura unternommen. Um den Lehrern Anreize zur Teilnahme zu geben, wurden drei Maßnahmen ergriffen:

- Ihnen wurde ein Fragebogen persönlich ausgehändigt und nach einer Woche auch wieder persönlich von den Probanden zurückgenommen.

- Den Probanden wurden die Ziele der Studie bei der Verteilung der Fragebögen erklärt, mit dem Hinweis auf die Bedeutung der Studie für den Sportunterricht in der arabischen, ägyptischen Republik, um möglichst viele richtige Antworten zu bekommen.

- Sie sollten ein Zeugnis der Sportfakultät der El Mansoura Universität erhalten, das darauf hinweist, dass sie bei der Untersuchung des Sportunterrichts im Bezirk El Dakahlia teilgenommen haben.

Daraus resultierte bei 200 verteilten Fragebogen eine Stichprobengröße von $\mathrm{N}=186$ (also einer Rücklaufquote von 93\%). So konnten etwa 25\% der Gesamtzahl der Sportlehrkräfte befragt werden. Genauer erfolgte dann eine Befragung von:

- 104 Sportlehrern der Mittelschule

- 17 Sportlehrerinnen der Mittelschule

- $54 \quad$ Sportlehrern der Oberschule und

- 11 Sportlehrerinnen der Oberschule 


\subsection{Datenverarbeitung}

Nach der Befragung, die im Zeitraum vom 29.12.2002 bis 07.01.2003 durchgeführt wurde, schlossen sich ab Mitte März 2003 an:

- Erstellung eines Codierplans,

- Übertragen der Daten auf Eingabeformulare,

- Eingabe der Daten in einen PC,

- Fehlersuche und -korrektur,

- Mit Hilfe eines Computerprogramms (SPSS Version 10.0.7) wurden die Daten ausgewertet. Hier wurden v.a. deskriptive Daten wie Mittelwerte und Häufigkeitsverteilungen generiert.

Im folgenden Kapitel werden die Ergebnisse dargestellt und diskutiert. 


\section{Darstellung und Diskussion der Ergebnisse}

Die erbrachten Ergebnisse der Untersuchungen seien hier dargestellt und diskutiert. Dies erfolgt zunächst sehr eng am Fragebogen. Dabei sei jedoch auf zwei Besonderheit zur Darstellungsform aufmerksam gemacht: 1. Sie erfolgt in großen Teilen in tabellarischer Form, die die Unterschiede zwischen Geschlechter und zwischen Schulformen deutlich machen soll. Wurden bei der Codierung der Items Likert-Skalen verwendet, so wird in den Tabellen nur noch die Codierung angegeben (1=stimme völlig zu, 2=stimme zu, 3=unentschieden, 4=stimme nicht zu, 5 =stimme überhaupt nicht zu). 2. Während im ersten Teil (Meinungen der Sportlehrerinnen und Sportlehrer) zunächst lediglich eine Darstellung der Ergebnisse erfolgt und diese dann im Anschluss zusammenfassend diskutiert werden, werden die einzelnen (Teil-) Ergebnisse zur tatsächlichen Umsetzung schon am Ende der Unterkategorien kritisch beleuctet. Danach erfolgt aber nochmals eine zusammenfassende Betrachtung. Schließlich werden die Ergebnisse der beiden Abschnitte ,Meinungen' und ,Praxis' konfrontiert und unter Berücksichtigung der strukturellen Bedingungen nochmals in seiner Gesamtheit diskutiert.

Abbildung 6 gibt zunächst einen Überblick zur Altersstruktur der untersuchten Sportlehrkräfte. Ersichtlich wird hier, dass die meisten im mittleren Alter sind und nur noch vergleichsweise wenig ältere Sportlehrer unterrichten, denn viele sind schon zum Bezirksleiter aufgestiegen. Ausnahmen bilden die Sportlehrer der Oberschule einerseits und die relativ jungen Sportlehrerinnen der Mittelschule.

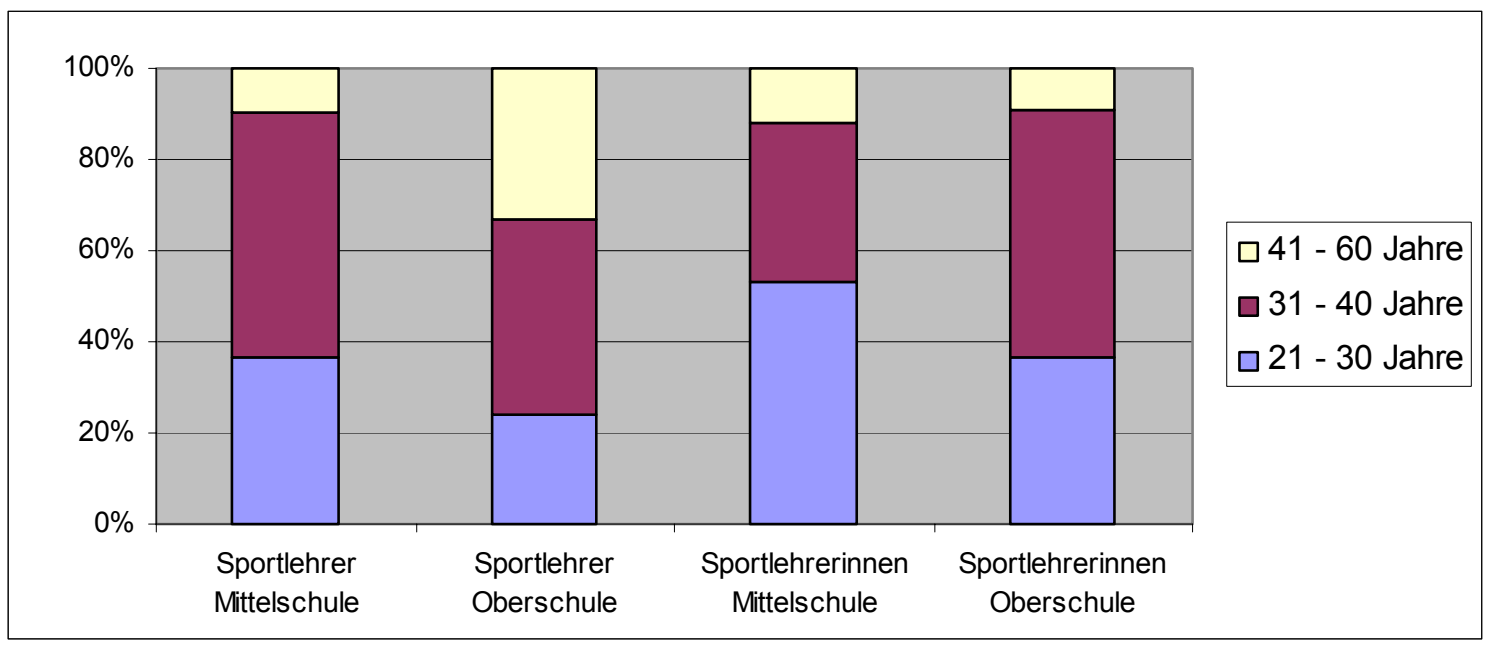

Abbildung 6: Altersverteilung der untersuchten Sportlehrkräfte. 


\subsection{Meinungen der Sportlehrerinnen und Sportlehrer}

Wie bereits erwähnt, wurden Sportlehrerinnen und Sportlehrer der Mittel- und Oberschulen befragt. Um den Überblick zu wahren, wird für die Unterkategorien jeweils eine Tabelle für die Mittelschulen und jeweils eine für Oberschulen abgebildet und in deren Anschluss kommentiert.

\subsubsection{Erziehungsziele}

Die Erziehungsziele des Sportunterrichts wurden in Kapitel 3.2 in sechs Unterkategorien zerlegt, nämlich Freizeit, Gesundheit, Sozialverhalten, psychische Eigenschaften, sportspezifische Kenntnisse und Freude/Spaß/Begeisterung (siehe im Überblick S. 62 unten). Im Folgenden werden die Ergebnisse aus den einzelnen Unterkategorien nacheinander dargestellt.

\section{Freizeit}

\begin{tabular}{|c|c|c|c|c|c|c|c|c|c|c|c|c|}
\hline \multirow{3}{*}{ Item } & \multicolumn{6}{|c|}{ Sportlehrer } & \multicolumn{6}{|c|}{ Sportlehrerinnen } \\
\hline & \multicolumn{5}{|c|}{ Häufigkeiten in \% } & \multirow{2}{*}{$\begin{array}{c}\text { Codierter } \\
\text { Mittelwert }\end{array}$} & \multicolumn{5}{|c|}{ Häufigkeiten in \% } & \multirow{2}{*}{$\begin{array}{l}\text { Codierter } \\
\text { Mittelwert }\end{array}$} \\
\hline & 1 & 2 & 3 & 4 & 5 & & 1 & 2 & 3 & 4 & 5 & \\
\hline $\begin{array}{l}1 \text { - Der Sportunterricht } \\
\text { ist ein wichtiges Mittel, } \\
\text { um die motorischen } \\
\text { Voraussetzungen für } \\
\text { den Freizeitsport zu } \\
\text { legen. }\end{array}$ & 56.7 & 23.1 & 1.0 & 9.6 & 9.6 & 1.92 & 58.8 & 17.6 & - & 11.8 & 11.8 & 2.00 \\
\hline $\begin{array}{l}2 \text { - Die Schüler sollen } \\
\text { viele verschiedene } \\
\text { Sportarten ausüben } \\
\text { können. }\end{array}$ & 71.2 & 27.9 & 1.05 & - & - & 1.30 & 82.4 & 17.6 & - & - & - & 1.18 \\
\hline $\begin{array}{l}3 \text { - Die Schüler sollen } \\
\text { ihren Freizeitsport } \\
\text { selbst organisieren } \\
\text { können. }\end{array}$ & 51.0 & 30.8 & 14.4 & 2.9 & 1.0 & 1.72 & 52.9 & 29.4 & 11.8 & - & 5.9 & 1.76 \\
\hline
\end{tabular}

Tabelle 10: Meinungen der Sportlehrkräfte der Mittelschule zur vorbereitenden Funktion des Sportunterrichts für den Freizeitsport. 


\begin{tabular}{|c|c|c|c|c|c|c|c|c|c|c|c|c|}
\hline \multirow{3}{*}{ Item } & \multicolumn{6}{|c|}{ Sportlehrer } & \multicolumn{6}{|c|}{ Sportlehrerinnen } \\
\hline & \multicolumn{5}{|c|}{ Häufigkeiten in \% } & \multirow{2}{*}{$\begin{array}{c}\text { Codierter } \\
\text { Mittelwert }\end{array}$} & \multicolumn{5}{|c|}{ Häufigkeiten in \% } & \multirow{2}{*}{$\begin{array}{l}\text { Codierter } \\
\text { Mittelwert }\end{array}$} \\
\hline & 1 & 2 & 3 & 4 & 5 & & 1 & 2 & 3 & 4 & 5 & \\
\hline $\begin{array}{l}1 \text { - Der Sportunterricht } \\
\text { ist ein wichtiges Mittel, } \\
\text { um die motorischen } \\
\text { Voraussetzungen für } \\
\text { den Freizeitsport zu } \\
\text { legen. }\end{array}$ & 68.5 & 16.7 & 1.9 & 5.6 & 7.4 & 1.67 & 63.6 & 27.3 & - & - & 9.1 & 1.64 \\
\hline $\begin{array}{l}2 \text { - Die Schüler sollen } \\
\text { viele verschiedene } \\
\text { Sportarten ausüben } \\
\text { können. }\end{array}$ & 61.1 & 38.9 & - & - & - & 1.39 & 45.5 & 54.5 & - & - & - & 1.55 \\
\hline $\begin{array}{l}3 \text { - Die Schüler sollen } \\
\text { ihren Freizeitsport } \\
\text { selbst organisieren } \\
\text { können. }\end{array}$ & 61.1 & 29.6 & 5.6 & 3.7 & - & 1.52 & 36.4 & 36.4 & 18.2 & 9.1 & - & 2.00 \\
\hline
\end{tabular}

Tabelle 11: Meinungen der Sportlehrkräfte der Oberschule zur vorbereitenden Funktion des Sportunterrichts für den Freizeitsport.

Aus den Tabellen 10 und 11 ist ersichtlich, dass sich sowohl Sportlehrerinnen als auch Sportlehrer beider Schulformen über die Funktion des Sportunterrichts für den Freizeitsport im Klaren sind. Sie messen diesem Erziehungsziel durchgehend eine hohe Bedeutung zu. Dies betrifft sowohl die motorischen und organisatorischen Voraussetzungen sowie eine große Bandbreite an Sportarten. Hervorzuheben ist allerdings, dass ein Teil der Sportlehrerinnen das Erziehungsziel Freizeit für ihre Schülerinnen ablehnen. Am stärksten zeigt sich dies bei den Sportlehrerinnen der Mittelschule, denn hier nehmen etwa ein Viertel der Befragten eine solche Haltung ein. 


\section{Gesundheit}

\begin{tabular}{|c|c|c|c|c|c|c|c|c|c|c|c|c|}
\hline \multirow{3}{*}{ Item } & \multicolumn{6}{|c|}{ Sportlehrer } & \multicolumn{6}{|c|}{ Sportlehrerinnen } \\
\hline & \multicolumn{5}{|c|}{ Häufigkeiten in \% } & \multirow{2}{*}{\begin{tabular}{|c|} 
Codierter \\
Mittelwert
\end{tabular}} & \multicolumn{5}{|c|}{ Häufigkeiten in \% } & \multirow{2}{*}{$\begin{array}{l}\text { Codierter } \\
\text { Mittelwert }\end{array}$} \\
\hline & 1 & 2 & 3 & 4 & 5 & & 1 & 2 & 3 & 4 & 5 & \\
\hline $\begin{array}{l}4 \text { - Die Schüler sollen } \\
\text { den Zusammenhang } \\
\text { von Bewegung und } \\
\text { Gesundheit kennen. }\end{array}$ & 77.9 & 21.2 & - & 1.0 & - & 1.24 & 76.5 & 17.6 & 5.9 & - & - & 1.29 \\
\hline $\begin{array}{l}5 \text { - Die Schüler sollen } \\
\text { den Zusammenhang } \\
\text { von Ernährung und } \\
\text { Gesundheit kennen. }\end{array}$ & 76.9 & 22.1 & - & 1.0 & - & 1.25 & 88.2 & 11.8 & - & - & - & 1.12 \\
\hline $\begin{array}{l}6 \text { - Die Schüler sollen } \\
\text { Kenntnisse über das } \\
\text { Herz-Kreislauf-System } \\
\text { haben. }\end{array}$ & 47.1 & 47.1 & 4.8 & 1.0 & - & 1.60 & 35.3 & 58.8 & 5.9 & - & - & 1.71 \\
\hline $\begin{array}{l}7 \text { - Die Schüler sollen } \\
\text { Kenntnisse über die } \\
\text { gesundheitliche Be- } \\
\text { deutung der Hygiene } \\
\text { haben. }\end{array}$ & 71.2 & 26.9 & - & 1.0 & 1.0 & 1.34 & 64.7 & 35.3 & - & - & - & 1.35 \\
\hline
\end{tabular}

Tabelle 12: Meinungen der Sportlehrkräfte der Mittelschule zur Vermittlung gesundheitlicher Kenntnisse durch den Sportunterricht.

\begin{tabular}{|c|c|c|c|c|c|c|c|c|c|c|c|c|}
\hline \multirow{3}{*}{ Item } & \multicolumn{6}{|c|}{ Sportlehrer } & \multicolumn{6}{|c|}{ Sportlehrerinnen } \\
\hline & \multicolumn{5}{|c|}{ Häufigkeiten in \% } & \multirow{2}{*}{$\begin{array}{c}\text { Codierter } \\
\text { Mittelwert }\end{array}$} & \multicolumn{5}{|c|}{ Häufigkeiten in \% } & \multirow{2}{*}{\begin{tabular}{|l} 
Codierter \\
Mittelwert \\
\end{tabular}} \\
\hline & 1 & 2 & 3 & 4 & 5 & & 1 & 2 & 3 & 4 & 5 & \\
\hline $\begin{array}{l}4 \text { - Die Schüler sollen } \\
\text { den Zusammenhang } \\
\text { von Bewegung und } \\
\text { Gesundheit kennen. }\end{array}$ & 88.9 & 7.4 & 3.7 & - & - & 1.15 & 63.6 & 36.4 & - & - & - & 1.36 \\
\hline $\begin{array}{l}5 \text { - Die Schüler sollen } \\
\text { den Zusammenhang } \\
\text { von Ernährung und } \\
\text { Gesundheit kennen. }\end{array}$ & 75.9 & 20.4 & 3.7 & - & - & 1.28 & 72.7 & 27.3 & - & - & - & 1.27 \\
\hline $\begin{array}{l}6 \text { - Die Schüler sollen } \\
\text { Kenntnisse über das } \\
\text { Herz-Kreislauf-System } \\
\text { haben. }\end{array}$ & 55.6 & 42.6 & 1.9 & - & - & 1.46 & 45.5 & 54.5 & - & - & - & 1.55 \\
\hline $\begin{array}{l}7 \text { - Die Schüler sollen } \\
\text { Kenntnisse über die } \\
\text { gesundheitliche Be- } \\
\text { deutung der Hygiene } \\
\text { haben. }\end{array}$ & 66.7 & 33.3 & - & - & - & 1.33 & 63.6 & 36.4 & - & - & - & 1.36 \\
\hline
\end{tabular}

Tabelle 13: Meinungen der Sportlehrkräfte der Oberschule zur Vermittlung gesundheitlicher Kenntnisse durch den Sportunterricht. 
Bei der Betrachtung der Tabellen 12 und 13 sieht man, dass die Sportlehrer der Vermittlung gesundheitlicher Kenntnisse durch ihren Unterricht durchschnittlich einen noch höheren Wert zuschreiben, als dies in der Erziehungskategorie Freizeit der Fall ist. Auch die Gesundheit als Erziehungsziel wird von Lehrern beider Geschlechter und beider Schulen als stark erstrebenswert erachtet. Interessant ist dabei der Aspekt, dass die Vermittlung von Kenntnissen zum Zusammenhang von Gesundheit und Bewegung im Durchschnitt höchste Zustimmungen erbrachte. Dieser stehen andere gesundheitliche Themen wie Ernährung oder Hygiene allerdings nur wenig nach. Die spezifischere Vermittlung von Kenntnissen über das Herz-Kreislauf-System erhält dabei die geringste Bewertung, erfährt aber immer noch eine sehr klare Zustimmung. Zusammen mit dem Befund, dass das Erziehungsziel gesundheitliche Kenntnisse insgesamt praktisch keine Ablehnung erfährt, bedeutet dies nun, dass die Vermittlung gesundheitlicher Kenntnisse als eine überaus wichtige Aufgabe für den eigenen Sportunterricht betrachtet wird.

\section{Sozialverhalten}

\begin{tabular}{|c|c|c|c|c|c|c|c|c|c|c|c|c|}
\hline \multirow{3}{*}{ Item } & \multicolumn{6}{|c|}{ Sportlehrer } & \multicolumn{6}{|c|}{ Sportlehrerinnen } \\
\hline & \multicolumn{5}{|c|}{ Häufigkeiten in \% } & \multirow{2}{*}{$\begin{array}{l}\text { Codierter } \\
\text { Mittelwert }\end{array}$} & \multicolumn{5}{|c|}{ Häufigkeiten in \% } & \multirow{2}{*}{$\begin{array}{l}\text { Codierter } \\
\text { Mittelwert }\end{array}$} \\
\hline & 1 & 2 & 3 & 4 & 5 & & 1 & 2 & 3 & 4 & 5 & \\
\hline $\begin{array}{l}8 \text { - Der Lehrer muss } \\
\text { Konflikte unter den } \\
\text { Schülern lösen. }\end{array}$ & 64.4 & 29.8 & 1.0 & 3.8 & 1.0 & 1.47 & 64.7 & 35.3 & - & - & - & 1.35 \\
\hline $\begin{array}{l}9 \text { - Die Schüler sollen } \\
\text { ihre Konflikte selbst } \\
\text { lösen }\end{array}$ & 25.0 & 39.4 & 10.6 & 23.1 & 1.9 & 2.37 & 29.4 & $\mid 17.6$ & 11.8 & 35.3 & 5.9 & 2.71 \\
\hline $\begin{array}{l}10 \text { - Die Schüler sollen } \\
\text { sprachliche Fähigkei- } \\
\text { ten zur Konfliktlösung } \\
\text { erlernen. }\end{array}$ & 26.0 & 52.9 & 18.3 & 1.9 & 1.0 & 1.99 & 35.3 & 58.8 & 5.9 & - & - & 1.71 \\
\hline $\begin{array}{l}11 \text { - Die Schüler sollen } \\
\text { Formen der Zusam- } \\
\text { menarbeit (z.B. Grup- } \\
\text { penarbeit) können. }\end{array}$ & 65.4 & 31.7 & 2.9 & - & - & 1.37 & 88.2 & 11.8 & - & - & - & 1.12 \\
\hline $\begin{array}{l}12 \text { - Die Schüler sollen } \\
\text { es akzeptieren lernen, } \\
\text { wenn sich ein anderer } \\
\text { durchsetzt. }\end{array}$ & 41.3 & 48.1 & 6.7 & 2.9 & 1.0 & 1.74 & 58.8 & 35.3 & - & 5.9 & - & 1.53 \\
\hline
\end{tabular}

Tabelle 14: Meinungen der Sportlehrkräfte der Mittelschule zum Erziehungsziel Sozialverhalten. 


\begin{tabular}{|c|c|c|c|c|c|c|c|c|c|c|c|c|}
\hline \multirow{3}{*}{ Item } & \multicolumn{6}{|c|}{ Sportlehrer } & \multicolumn{6}{|c|}{ Sportlehrerinnen } \\
\hline & \multicolumn{5}{|c|}{ Häufigkeiten in \% } & \multirow{2}{*}{$\begin{array}{l}\text { Codierter } \\
\text { Mittelwert } \\
\end{array}$} & \multicolumn{5}{|c|}{ Häufigkeiten in \% } & \multirow{2}{*}{$\begin{array}{l}\text { Codierter } \\
\text { Mittelwert }\end{array}$} \\
\hline & 1 & 2 & 3 & 4 & 5 & & 1 & 2 & 3 & 4 & 5 & \\
\hline $\begin{array}{l}8 \text { - Der Lehrer muss } \\
\text { Konflikte unter den } \\
\text { Schülern lösen. }\end{array}$ & 63.0 & 31.5 & 5.6 & - & - & 1.43 & 63.6 & 27.3 & - & - & 9.1 & 1.64 \\
\hline $\begin{array}{l}9 \text { - Die Schüler sollen } \\
\text { ihre Konflikte selbst } \\
\text { lösen }\end{array}$ & 25.9 & 40.7 & 7.4 & 25.9 & - & 2.33 & 27.3 & 27.3 & 9.1 & 18.2 & 18.2 & 2.73 \\
\hline $\begin{array}{l}10 \text { - Die Schüler sollen } \\
\text { sprachliche Fähigkei- } \\
\text { ten zur Konfliktlösung } \\
\text { erlernen. }\end{array}$ & 24.1 & 72.2 & - & 3.7 & - & 1.83 & 45.5 & 45.5 & 9.1 & - & - & 1.64 \\
\hline $\begin{array}{l}11 \text { - Die Schüler sollen } \\
\text { Formen der Zusam- } \\
\text { menarbeit (z.B. Grup- } \\
\text { penarbeit) können. }\end{array}$ & 74.1 & 25.9 & - & - & - & 1.26 & 72.7 & 27.3 & - & - & - & 1.27 \\
\hline $\begin{array}{l}12 \text { - Die Schüler sollen } \\
\text { es akzeptieren lernen, } \\
\text { wenn sich ein anderer } \\
\text { durchsetzt. }\end{array}$ & 50.0 & 44.4 & - & 5.6 & - & 1.61 & 45.5 & 45.5 & 9.1 & - & - & 1.64 \\
\hline
\end{tabular}

Tabelle 15: Meinungen der Sportlehrkräfte der Oberschule zum Erziehungsziel Sozialverhalten.

Anhand der Tabellen 14 und 15 ist ersichtlich, dass die Sportlehrkräfte die Grundlagen für Sozialverhalten wie sprachliche Fähigkeiten zur Konfliktlösung, Zusammenarbeit und Akzeptanz der Erfolge Anderer durchgehend für wichtig bis sehr wichtig halten. Als Erziehungsziele werden diese Grundlagen von den Lehrkräften der Oberschulen geringfügig klarer erkannt. Treten dann allerdings Konflikte auf, so sind die Antwortformate bezüglich der Lösungsstrategien widersprüchlich. Während die Konfliktlösung durch die Lehrer eine hohe Zustimmung erfährt, zeigt sich kein umgekehrter Effekt bei der Konfliktlösung durch die Schüler selbst. Zum Teil lässt sich eine klare Ablehnung selbständiger Problemlösungen als Erziehungsziel ausmachen (bei den Sportlehrerinnen mit etwa 40\% sogar höher als bei den Sportlehrern mit ca. 25\%), jedoch stimmen dem die Sportlehrkräfte auch deutlich zu. Daraus kann einerseits gefolgert werden, dass die Konfliktlösung durch die Lehrer als klarer Auftrag verstanden wird, jedoch in manchen Fällen den Schülern überlassen sein sollte. Andererseits kann aber auch vermutet werden, dass bei den Sportlehrkräften Unklarheit darüber besteht, wie mit Konflikten umgegangen werden sollte. 


\begin{tabular}{|c|c|c|c|c|c|c|c|c|c|c|c|c|}
\hline \multirow{3}{*}{ Item } & \multicolumn{6}{|c|}{ Sportlehrer } & \multicolumn{6}{|c|}{ Sportlehrerinnen } \\
\hline & \multicolumn{5}{|c|}{ Häufigkeiten in \% } & \multirow{2}{*}{$\begin{array}{l}\text { Codierter } \\
\text { Mittelwert } \\
\end{array}$} & \multicolumn{5}{|c|}{ Häufigkeiten in \% } & \multirow{2}{*}{$\begin{array}{l}\text { Codierter } \\
\text { Mittelwert }\end{array}$} \\
\hline & 1 & 2 & 3 & 4 & 5 & & 1 & 2 & 3 & 4 & 5 & \\
\hline $\begin{array}{l}13 \text { - Die Schüler sollen } \\
\text { Selbstvertrauen } \\
\text { bekommen. }\end{array}$ & 69.2 & 29.8 & 1.0 & - & - & 1.32 & 76.5 & 23.5 & - & - & - & 1.24 \\
\hline $\begin{array}{l}14 \text { - Die Schüler sol- } \\
\text { len,Kampgeist“ er- } \\
\text { werben. }\end{array}$ & 67.3 & 29.8 & 1.0 & 1.9 & - & 1.38 & 82.4 & 17.6 & - & - & - & 1.18 \\
\hline $\begin{array}{l}15 \text { - Die Schüler sollen } \\
\text { Führungsqualitäten } \\
\text { erwerben. }\end{array}$ & 76.0 & 22.1 & - & 1.9 & - & 1.28 & 88.9 & 11.1 & - & - & - & 1.18 \\
\hline $\begin{array}{l}16 \text { - Die Schüler sollen } \\
\text { Gehorsam bei Anwei- } \\
\text { sungen und Befehlen } \\
\text { lernen. }\end{array}$ & 78.8 & 18.3 & 2.9 & - & - & 1.24 & 88.2 & 11.8 & - & - & - & 1.12 \\
\hline
\end{tabular}

Tabelle 16: Meinungen der Sportlehrkräfte der Mittelschule zur Förderung psychischer Eigenschaften.

\begin{tabular}{|c|c|c|c|c|c|c|c|c|c|c|c|c|}
\hline \multirow{3}{*}{ Item } & \multicolumn{6}{|c|}{ Sportlehrer } & \multicolumn{6}{|c|}{ Sportlehrerinnen } \\
\hline & \multicolumn{5}{|c|}{ Häufigkeiten in \% } & \multirow{2}{*}{$\begin{array}{l}\text { Codierter } \\
\text { Mittelwert }\end{array}$} & \multicolumn{5}{|c|}{ Häufigkeiten in $\%$} & \multirow{2}{*}{\begin{tabular}{|l} 
Codierter \\
Mitte/wert
\end{tabular}} \\
\hline & 1 & 2 & 3 & 4 & 5 & & 1 & 2 & 3 & 4 & 5 & \\
\hline $\begin{array}{l}13 \text { - Die Schüler sollen } \\
\text { Selbstvertrauen } \\
\text { bekommen. }\end{array}$ & 70.4 & 29.6 & - & - & - & 1.30 & 72.7 & 27.3 & - & - & - & 1.27 \\
\hline $\begin{array}{l}14 \text { - Die Schüler sollen } \\
\text { „Kampgeist" er- } \\
\text { werben. }\end{array}$ & 83.3 & 16.7 & - & - & - & 1.17 & 63.6 & 36.4 & - & - & - & 1.36 \\
\hline $\begin{array}{l}15 \text { - Die Schüler sollen } \\
\text { Führungsqualitäten } \\
\text { erwerben. }\end{array}$ & 88.9 & 11.1 & - & - & - & 1.11 & 63.6 & 27.3 & - & 9.1 & - & 1.55 \\
\hline $\begin{array}{l}16 \text { - Die Schüler sollen } \\
\text { Gehorsam bei Anwei- } \\
\text { sungen und Befehlen } \\
\text { lernen. }\end{array}$ & 87.0 & 11.1 & 1.9 & - & - & 1.15 & 90.9 & 9.1 & - & - & - & 1.09 \\
\hline
\end{tabular}

Tabelle 17: Meinungen der Sportlehrkräfte der Oberschule zur Förderung psychischer Eigenschaften.

Bezüglich des Erziehungszieles der Förderung psychischer Eigenschaften durch den Sportunterricht herrscht laut Tabellen 16 und 17 weitgehende Einigkeit unter den Sportlehrkräften. Hier besteht eine überaus starke Zustimmung, die die Werte zur Vermittlung gesundheitlicher Kenntnisse durchschnittlich sogar nochmals übersteigt. Mit einem einzigen Ausreißer bei der Führungsqualität (Sportlehrerinnen der Oberschule) lehnen die Sportlehrkräfte diese Erziehungsziele praktisch 
nicht ab und sind auch kaum unentschieden. Selbstvertrauen und „Kampfgeist“ als Bedingungen für ein leistungssportliches Sportengagement spiegeln das Verständnis des Sports in Ägypten wider und machen deutlich, dass der Sportunterricht aus Sicht der Sportlehrkräfte eben genau diese Ziele verfolgen sollte. Ganz ähnlich können die Aspekte Führungsqualität und Gehorsam als Spiegel des kulturellen Hintergrundes verstanden werden.

\section{Sportspezifische Kenntnisse}

\begin{tabular}{|c|c|c|c|c|c|c|c|c|c|c|c|c|}
\hline \multirow{3}{*}{ Item } & \multicolumn{6}{|c|}{ Sportlehrer } & \multicolumn{6}{|c|}{ Sportlehrerinnen } \\
\hline & \multicolumn{5}{|c|}{ Häufigkeiten in \% } & \multirow{2}{*}{$\begin{array}{l}\text { Codierter } \\
\text { Mittelwert }\end{array}$} & \multicolumn{5}{|c|}{ Häufigkeiten in \% } & \multirow{2}{*}{$\begin{array}{l}\text { Codierter } \\
\text { Mittelwert }\end{array}$} \\
\hline & 1 & 2 & 3 & 4 & 5 & & 1 & 2 & 3 & 4 & 5 & \\
\hline $\begin{array}{l}17 \text { - Die Schüler sollen } \\
\text { die räumlichen und / } \\
\text { oder gerätebezogenen } \\
\text { Regeln bei den unter- } \\
\text { schiedlichen gelehrten } \\
\text { Sportarten wissen. }\end{array}$ & 41.3 & 51.0 & 3.8 & 3.8 & - & 1.70 & 29.4 & 64.7 & - & 5.9 & - & 1.82 \\
\hline $\begin{array}{l}18 \text { - Die Schüler sollen } \\
\text { die Spielregeln und } \\
\text { taktischen Regeln in } \\
\text { den unterunterschied- } \\
\text { lichen Sportarten ken- } \\
\text { nen und einhalten. }\end{array}$ & 20.2 & 59.6 & 10.6 & 9.6 & - & 2.10 & 23.5 & 64.7 & - & 11.8 & - & 2.00 \\
\hline $\begin{array}{l}19 \text { - Die Schüler sollen } \\
\text { über wichtige aktuelle } \\
\text { Sportereignisse (z.B. } \\
\text { Fußballweltmeister- } \\
\text { schaft, Olympische } \\
\text { Spiele) informiert wer- } \\
\text { den. }\end{array}$ & 34.6 & 50.0 & 10.6 & 3.8 & 1.0 & 1.87 & 41.2 & 58.8 & - & - & - & 1.59 \\
\hline $\begin{array}{l}20 \text { - Die Schüler sollen } \\
\text { möglichst viele eigene } \\
\text { Lernerfahrungen } \\
\text { sammeln. }\end{array}$ & 41.3 & 31.7 & 13.5 & 12.5 & 1.0 & 2.00 & 47.1 & 23.5 & 11.8 & 17.6 & - & 2.00 \\
\hline
\end{tabular}

Tabelle 18: Meinungen der Sportlehrkräfte der Mittelschule zur Vermittlung sportspezifischer Kenntnisse. 


\begin{tabular}{|c|c|c|c|c|c|c|c|c|c|c|c|c|}
\hline \multirow{3}{*}{ Item } & \multicolumn{6}{|c|}{ Sportlehrer } & \multicolumn{6}{|c|}{ Sportlehrerinnen } \\
\hline & \multicolumn{5}{|c|}{ Häufigkeiten in \% } & \multirow{2}{*}{$\begin{array}{l}\text { Codierter } \\
\text { Mittelwert }\end{array}$} & \multicolumn{5}{|c|}{ Häufigkeiten in \% } & \multirow{2}{*}{$\begin{array}{l}\text { Codierter } \\
\text { Mittelwert }\end{array}$} \\
\hline & 1 & 2 & 3 & 4 & 5 & & 1 & 2 & 3 & 4 & 5 & \\
\hline $\begin{array}{l}17 \text { - Die Schüler sollen } \\
\text { die räumlichen und } \\
\text { loder gerätebezoge- } \\
\text { nen Regeln bei den } \\
\text { unterschiedlichen } \\
\text { gelehrten Sportarten } \\
\text { wissen. }\end{array}$ & 50.0 & 48.1 & 1.9 & - & - & 1.52 & 45.5 & 45.5 & 9.0 & - & - & 1.64 \\
\hline $\begin{array}{l}18 \text { - Die Schüler sollen } \\
\text { die Spielregeln und } \\
\text { taktischen Regeln in } \\
\text { den unterunterschied- } \\
\text { lichen Sportarten ken- } \\
\text { nen und einhalten. }\end{array}$ & 16.7 & 70.4 & 3.7 & 9.3 & - & 2.06 & 27.3 & 45.5 & 18.2 & 9.1 & - & 2.09 \\
\hline $\begin{array}{l}19 \text { - Die Schüler sollen } \\
\text { über wichtige aktuelle } \\
\text { Sportereignisse (z.B. } \\
\text { Fußballweltmeister- } \\
\text { schaft, Olympische } \\
\text { Spiele) informiert wer- } \\
\text { den. }\end{array}$ & 51.9 & 38.9 & 7.4 & 1.9 & - & 1.59 & 36.4 & 54.5 & 9.1 & - & - & 1.73 \\
\hline $\begin{array}{l}20 \text { - Die Schüler sollen } \\
\text { möglichst viele eigene } \\
\text { Lernerfahrungen } \\
\text { sammeln. }\end{array}$ & 44.4 & 40.7 & - & 14.8 & - & 1.85 & 18.2 & 63.6 & 9.1 & 9.1 & - & 2.09 \\
\hline
\end{tabular}

Tabelle 19: Meinungen der Sportlehrkräfte der Oberschule zur Vermittlung sportspezifischer Kenntnisse.

Die Tabellen zur Vermittlung sportspezifischer Kenntnisse (18 und 19) zeigen durchschnittlich eine Konzentration um den Wert 2, wodurch eine Zustimmung zum Ausdruck kommt. In den meisten Fällen liegt hier auch der Höhepunkt der Häufigkeitsverteilungen. Die geringsten Bewertungen (aber immer noch Zustimmung) erhalten die Spielregeln / taktische Regeln der Sportarten und die eigenen Lernerfahrungen der Schüler. Letztere treffen zum Teil sogar auf Ablehnung. Dieser Befund scheint in die gleiche Richtung zu weisen, wie die Konfliktlösung im Erziehungsbereich Sozialverhalten, d.h. es scheint eine Unklarheit oder aber eine situationsabhängige Beurteilung vorzuliegen. Etwas deutlicher fällt die Zustimmung bei der Kenntnisvermittlung in Bezug auf Geräte und Spielfelder aus. Hier scheint schon durch den Lehrplan eine feste Zielbindung durch die Sportlehrkräfte stattgefunden zu haben. Interessant ist weiterhin, dass dies in etwa die gleichen Werte erreicht wie die Informationen zu aktuellen Sportereignissen. SportlehrerInnen in Ägypten scheinen weiterhin ihrer Vorbildfunktion nachkommen und ihrer Begeisterung für den Sport Ausdruck verleihen zu wollen. Als Erziehungsziel 
scheinen die Vermittlung sportspezifischer Kenntnisse durch ihre Vorgabe zwar auf Zustimmung zu treffen, jedoch sind sie nach den Daten in den Köpfen nicht so stark verankert wie Gesundheit oder psychische Eigenschaften, die enger mit dem Charakter denn der Reglementierung des Sporttreibens verbunden sind.

\section{Freude/Spaß/Begeisterung}

\begin{tabular}{|c|c|c|c|c|c|c|c|c|c|c|c|c|}
\hline \multirow{3}{*}{ Item } & \multicolumn{6}{|c|}{ Sportlehrer } & \multicolumn{6}{|c|}{ Sportlehrerinnen } \\
\hline & \multicolumn{5}{|c|}{ Häufigkeiten in \% } & \multirow{2}{*}{$\begin{array}{l}\text { Codierter } \\
\text { Mittelwert }\end{array}$} & \multicolumn{5}{|c|}{ Häufigkeiten in \% } & \multirow{2}{*}{$\begin{array}{l}\text { Codierter } \\
\text { Mittelwert }\end{array}$} \\
\hline & 1 & 2 & 3 & 4 & 5 & & 1 & 2 & 3 & 4 & 5 & \\
\hline $\begin{array}{l}21 \text { - Der Lehrer sollte } \\
\text { möglichst viele positi- } \\
\text { ve Rückmeldungen } \\
\text { geben, um die Schüler } \\
\text { stolz auf ihre sportli- } \\
\text { chen Handlungen zu } \\
\text { machen. }\end{array}$ & 75.0 & 24.0 & 1.0 & - & - & 1.26 & 82.4 & 17.6 & - & - & - & 1.18 \\
\hline $\begin{array}{l}22 \text { - Der Lehrer sollte } \\
\text { immer Hilfe anbieten. }\end{array}$ & 55.8 & 30.8 & 9.6 & 3.8 & - & 1.62 & 70.6 & 29.4 & - & - & - & 1.29 \\
\hline $\begin{array}{l}23 \text { - Der Lehrer sollte } \\
\text { ein gutes Vorbild sein } \\
\text { indem er seine Be- } \\
\text { geisterung für den } \\
\text { Sport zum Ausdruck } \\
\text { bringt. }\end{array}$ & 61.5 & 32.7 & 2.9 & 1.9 & 1.0 & 1.48 & 52.9 & 47.1 & - & - & - & 1.47 \\
\hline $\begin{array}{l}24 \text { - Die Bedürfnisse } \\
\text { der Schüler sind zu } \\
\text { berücksichtigen. }\end{array}$ & 57.7 & 38.5 & 3.8 & - & - & 1.46 & 41.2 & 58.8 & - & - & - & 1.59 \\
\hline
\end{tabular}

Tabelle 20: Meinungen der Sportlehrkräfte der Mittelschule zur Vermittlung von Freude/ Spaß/ Begeisterung. 


\begin{tabular}{|c|c|c|c|c|c|c|c|c|c|c|c|c|}
\hline \multirow{3}{*}{ Item } & \multicolumn{6}{|c|}{ Sportlehrer } & \multicolumn{6}{|c|}{ Sportlehrerinnen } \\
\hline & \multicolumn{5}{|c|}{ Häufigkeiten in \% } & \multirow{2}{*}{$\begin{array}{l}\text { Codierter } \\
\text { Mittelwert } \\
\end{array}$} & \multicolumn{5}{|c|}{ Häufigkeiten in \% } & \multirow{2}{*}{$\begin{array}{l}\text { Codierter } \\
\text { Mittelwert } \\
\end{array}$} \\
\hline & 1 & 2 & 3 & 4 & 5 & & 1 & 2 & 3 & 4 & 5 & \\
\hline $\begin{array}{l}21 \text { - Der Lehrer sollte } \\
\text { möglichst viele positi- } \\
\text { ve Rückmeldungen } \\
\text { geben, um die Schüler } \\
\text { stolz auf ihre sportli- } \\
\text { chen Handlungen zu } \\
\text { machen. }\end{array}$ & 72.2 & 27.8 & - & - & - & 1.28 & 81.8 & $\mid 18.2$ & - & - & - & 1.18 \\
\hline $\begin{array}{l}22 \text { - Der Lehrer sollte } \\
\text { immer Hilfe anbieten. }\end{array}$ & 53.7 & 42.6 & 1.9 & - & 1.9 & 1.54 & 63.6 & 27.3 & 9.1 & - & - & 1.45 \\
\hline $\begin{array}{l}23 \text { - Der Lehrer sollte } \\
\text { ein gutes Vorbild sein } \\
\text { indem er seine Be- } \\
\text { geisterung für den } \\
\text { Sport zum Ausdruck } \\
\text { bringt. }\end{array}$ & 59.3 & 35.2 & 1.9 & 3.7 & - & 1.50 & 54.5 & 36.4 & 9.1 & - & - & 1.55 \\
\hline $\begin{array}{l}24 \text { - Die Bedürfnisse } \\
\text { der Schüler sind zu } \\
\text { berücksichtigen. }\end{array}$ & 46.3 & 46.3 & 1.9 & 5.6 & - & 1.67 & 63.6 & 36.4 & - & - & - & 1.36 \\
\hline
\end{tabular}

Tabelle 21: Meinungen der Sportlehrkräfte der Oberschule zur Vermittlung von Freude/ Spaß/ Begeisterung.

Die Tabellen 20 und 21 zeigen insgesamt (d.h. über Geschlechter und Schulformen hinweg) eine starke Zustimmung und praktisch keine Ablehnung zu Aspekten des Erziehungszieles Freude/Spaß/Begeisterung. Vergleichbar ist der Grad der Zustimmung mit dem Erziehungsbereich gesundheitliche Kenntnisse. Auch im Rahmen dieses Erziehungszieles sind die Unterschiede nur sehr gering. Etwas stärker als Sportlehrer möchten Sportlehrerinnen Hilfe geben und votieren ebenfalls sehr bestimmt für die Überzeugung, möglichst viele Rückmeldungen zu geben. Umgekehrt ist die Zustimmung zur Vorbildfunktion des Sportlehrers bei den männlichen Sportlehrkräften geringfügig stärker.

\subsubsection{Methodisches Vorgehen}

Das unter methodischem Vorgehen bezeichnete Agieren der Sportlehrer in ihrem Unterricht wurde in Kapitel 3.6 in vier Unterkategorien zerlegt. Die Ergebnisse zu diesen einzelnen Aspekte werden nun in nachfolgender Reihenfolge dargestellt: Allgemeine Methodik, Methodik bei der sportartspezifischen Fertigkeitsvermittlung, inhaltliche Schwerpunkte und Bewertungsmethoden. 


\section{Allgemeine Methodik}

\begin{tabular}{|c|c|c|c|c|c|c|c|c|c|c|c|c|}
\hline \multirow{3}{*}{ Item } & \multicolumn{6}{|c|}{ Sportlehrer } & \multicolumn{6}{|c|}{ Sportlehrerinnen } \\
\hline & \multicolumn{5}{|c|}{ Häufigkeiten in \% } & \multirow{2}{*}{$\begin{array}{c}\text { Codierter } \\
\text { Mittelwert } \\
\end{array}$} & \multicolumn{5}{|c|}{ Häufigkeiten in \% } & \multirow{2}{*}{$\begin{array}{l}\text { Codierter } \\
\text { Mittelwert } \\
\end{array}$} \\
\hline & 1 & 2 & 3 & 4 & 5 & & 1 & 2 & 3 & 4 & 5 & \\
\hline $\begin{array}{l}25 \text { - Es ist wichtig, } \\
\text { dass der Lehrer in der } \\
\text { Praxis immer auch die } \\
\text { verschiedenen Übun- } \\
\text { gen vormacht. }\end{array}$ & 30.8 & 66.3 & 1.9 & 1.0 & - & 1.73 & 23.5 & 76.5 & - & - & - & 1.76 \\
\hline $\begin{array}{l}26 \text { - Der Lehrer muss } \\
\text { die Schüler anleiten. } \\
\text { Das muss in jeder } \\
\text { Situation so sein. }\end{array}$ & 58.7 & 37.5 & 1.0 & 2.9 & - & 1.48 & 58.8 & 35.3 & - & 5.9 & - & 1.53 \\
\hline $\begin{array}{l}27 \text { - In den überwie- } \\
\text { genden Sportspielsi- } \\
\text { tuationen sollen die } \\
\text { Schüler (u.a. mit Hilfe } \\
\text { des Lehrers) lernen, } \\
\text { selbständig Problem- } \\
\text { lösungen zu finden. }\end{array}$ & 40.4 & 44.2 & 9.6 & 4.8 & 1.0 & 1.82 & 17.6 & 64.7 & 11.8 & 5.9 & - & 2.06 \\
\hline $\begin{array}{l}28 \text { - Der Lehrer soll } \\
\text { immer Rückmeldun- } \\
\text { gen (richtig - falsch) } \\
\text { während der Ausbil- } \\
\text { dung geben. }\end{array}$ & 60.6 & 33.7 & 2.9 & 1.9 & 1.0 & 1.49 & 64.7 & 35.3 & - & - & - & 1.35 \\
\hline $\begin{array}{l}29 \text { - Prüfungen sollten } \\
\text { nicht nur für den Leh- } \\
\text { rer sein, sondern auch } \\
\text { eine wichtige Metho- } \\
\text { den, um den Lerner- } \\
\text { folg der Schüler zu } \\
\text { sichern. }\end{array}$ & 89.4 & 10.6 & - & - & - & 1.11 & 94.1 & 5.9 & - & - & - & 1.16 \\
\hline $\begin{array}{l}30 \text { - Die Schüler sollen } \\
\text { sich selbständig (im } \\
\text { Rahmen der Ausbil- } \\
\text { dung) auf die Prüfung } \\
\text { vorbereiten. }\end{array}$ & 39.4 & 40.4 & 17.3 & 2.9 & - & 1.84 & 52.9 & 29.4 & 11.8 & 5.9 & - & 1.71 \\
\hline $\begin{array}{l}\text { 31- Wettkämpfe mit } \\
\text { anderen Schulen sind } \\
\text { wichtig. }\end{array}$ & 64.4 & 33.7 & 1.0 & 1.0 & - & 1.38 & 82.4 & 17.6 & - & - & - & 1.18 \\
\hline $\begin{array}{l}32 \text { - Die Schüler sollen } \\
\text { in allen Sportarten } \\
\text { Wettkämpfe durchfüh- } \\
\text { ren. }\end{array}$ & 26.0 & 57.7 & 5.8 & 6.7 & 3.8 & 2.05 & 23.5 & 64.7 & - & 11.8 & - & 2.00 \\
\hline $\begin{array}{l}33 \text { - Die Schüler sollen } \\
\text { in jedem Fall sportge- } \\
\text { recht gekleidet sein. }\end{array}$ & 75.0 & 24.0 & 1.0 & - & - & 1.26 & 70.6 & 29.4 & - & - & - & 1.29 \\
\hline
\end{tabular}

Tabelle 22: Meinungen der Sportlehrkräfte der Mittelschule zur allgemeinen Methodik. 


\begin{tabular}{|c|c|c|c|c|c|c|c|c|c|c|c|c|}
\hline \multirow{3}{*}{ Item } & \multicolumn{6}{|c|}{ Sportlehrer } & \multicolumn{6}{|c|}{ Sportlehrerinnen } \\
\hline & \multicolumn{5}{|c|}{ Häufigkeiten in \% } & \multirow{2}{*}{\begin{tabular}{|c|} 
Codierter \\
Mittelwert
\end{tabular}} & \multicolumn{5}{|c|}{ Häufigkeiten in \% } & \multirow{2}{*}{$\begin{array}{l}\text { Codierter } \\
\text { Mittelwert } \\
\end{array}$} \\
\hline & 1 & 2 & 3 & 4 & 5 & & 1 & 2 & 3 & 4 & 5 & \\
\hline $\begin{array}{l}25 \text { - Es ist wichtig, } \\
\text { dass der Lehrer in der } \\
\text { Praxis immer auch die } \\
\text { verschiedenen Übun- } \\
\text { gen vormacht. }\end{array}$ & 48.1 & 50.0 & 1.9 & - & - & 1.54 & 63.6 & 36.4 & - & - & - & 1.36 \\
\hline $\begin{array}{l}26 \text { - Der Lehrer muss } \\
\text { die Schüler anleiten. } \\
\text { Das muss in jeder } \\
\text { Situation so sein. }\end{array}$ & 66.7 & 27.8 & 3.7 & - & 1.9 & 1.43 & 72.7 & 27.3 & - & - & - & 1.27 \\
\hline $\begin{array}{l}27 \text { - In den überwie- } \\
\text { genden Sportspielsi- } \\
\text { tuationen sollen die } \\
\text { Schüler (u.a. mit Hilfe } \\
\text { des Lehrers) lernen, } \\
\text { selbständig Problem- } \\
\text { lösungen zu finden. }\end{array}$ & 59.3 & 33.3 & 3.7 & 3.7 & - & 1.52 & 27.3 & 45.5 & 9.1 & 18.2 & - & 2.18 \\
\hline $\begin{array}{l}28 \text { - Der Lehrer soll } \\
\text { immer Rückmeldun- } \\
\text { gen (richtig - falsch) } \\
\text { während der Ausbil- } \\
\text { dung geben. }\end{array}$ & 75.9 & 24.1 & - & - & - & 1.24 & 63.6 & 27.3 & - & 9.1 & - & 1.55 \\
\hline $\begin{array}{l}29 \text { - Prüfungen sollten } \\
\text { nicht nur für den Leh- } \\
\text { rer sein, sondern auch } \\
\text { eine wichtige Metho- } \\
\text { den, um den Lerner- } \\
\text { folg der Schüler zu } \\
\text { sichern. }\end{array}$ & 87.0 & 11.1 & 1.9 & - & - & 1.15 & 100 & - & - & - & - & 1.00 \\
\hline $\begin{array}{l}30 \text { - Die Schüler sollen } \\
\text { sich selbständig (im } \\
\text { Rahmen der Ausbil- } \\
\text { dung) auf die Prüfung } \\
\text { vorbereiten. }\end{array}$ & 61.1 & 24.1 & 14.8 & - & - & 1.54 & 54.5 & 27.3 & 18.2 & - & - & 1.64 \\
\hline $\begin{array}{l}\text { 31- Wettkämpfe mit } \\
\text { anderen Schulen sind } \\
\text { wichtig. }\end{array}$ & 83.3 & 11.1 & 3.7 & - & 1.9 & 1.26 & 90.9 & 9.1 & - & - & - & 1.09 \\
\hline $\begin{array}{l}32 \text { - Die Schüler sollen } \\
\text { in allen Sportarten } \\
\text { Wettkämpfe durchfüh- } \\
\text { ren. }\end{array}$ & 33.3 & 46.3 & 5.6 & 11.1 & 3.7 & 2.06 & 36.4 & 36.4 & 9.1 & 9.1 & 9.1 & 2.18 \\
\hline $\begin{array}{l}33 \text { - Die Schüler sollen } \\
\text { in jedem Fall sportge- } \\
\text { recht gekleidet sein. }\end{array}$ & 64.8 & 31.5 & 1.9 & 1.9 & - & 1.41 & 90.9 & 9.1 & - & - & - & 1.09 \\
\hline
\end{tabular}

Tabelle 23: Meinungen der Sportlehrkräfte der Oberschule zur allgemeinen Methodik.

Auch die in den Tabellen 22 und 23 zusammengefassten Ergebnisse zur allgemeinen Methodik zeigen insgesamt eine hohe Zustimmung in den verschiedenen 
Aspekten. Wiederum zeigen sich lediglich nur geringste Unterschiede zwischen Sportlehrerinnen und Sportlehrern sowie zwischen den Schulformen. Während der Höhepunkt der Häufigkeitsverteilungen meist bei voller Zustimmung liegt, so ist dies am deutlichsten bei dem Aspekt, dass Prüfungen zur Sicherung des Lernerfolges der Schüler dienen sollten. Sehr wichtig für die Lehrkräfte ist ebenfalls, die Schüler entsprechend anzuleiten (und die Übungen vorzumachen), einfache Rückmeldungen zu geben, Wettkämpfe mit anderen Schulen und eine sportgerechte Bekleidung der Schüler. Dies scheint insgesamt bei den Lehrerinnen und auch bei den Lehrkräften der Oberschulen tendenziell stärker ausgeprägt. Mit Blick auf die Oberschule wird daher vorsichtig vermutet, dass die Leistungsorientierung eine größere Rolle spielt als in den Mittelschulen. Obwohl die mit selbständigem Handeln der Schüler verbundenen Aspekte (Problemlösungen und Prüfungsvorbereitung) sowie die Durchführung von Wettkämpfen in allen Sportarten insgesamt auch eine Zustimmung erfahren, treffen diese Items teilweise auf Ablehnung. Auch hier ist die Ablehrung der weiblichen Lehrkräfte geringfügig klarer. Ähnlich wie bei den Erziehungszielen spiegelt auch die allgemeine Methodik insgesamt ein leistungsorientiertes Bild vom Sport und eine leichte Unsicherheit oder situationsabhängige Beurteilung in Bezug auf selbständiges Handeln der Schüler wider.

\section{Methodik bei der sportartspezifischen Fertigkeitsvermittlung}

\begin{tabular}{|c|c|c|c|}
\hline \multirow{2}{*}{ Item } & \multirow{2}{*}{ Antwortmöglichkeiten } & Sportlehrer & Sportlehrerinnen \\
\hline & & Häufigkeiten in \% & Häufigkeiten in \% \\
\hline \multirow{8}{*}{$\begin{array}{l}34 \text { - Welche Lehrmetho- } \\
\text { den passen bei der Ver- } \\
\text { mittlung sportartspezifi- } \\
\text { scher Fertigkeiten zu Ihren } \\
\text { Schülern? }\end{array}$} & Nur Ganzheitsmethode & 6.7 & - \\
\hline & Nur Teilmethode & 2.9 & - \\
\hline & Nur Ganz-Teil-Methode & 19.2 & 5.9 \\
\hline & $\begin{array}{l}\text { Sowohl Ganzheitsmethode als } \\
\text { auch Teilmethode }\end{array}$ & 34.6 & 47.1 \\
\hline & $\begin{array}{l}\text { Sowohl Ganzheitsmethode als } \\
\text { auch Ganz-Teil-Methode }\end{array}$ & 2.9 & 11.8 \\
\hline & $\begin{array}{l}\text { Sowohl Teilmethode als auch } \\
\text { Ganz-Teil-Methode }\end{array}$ & 12.5 & 11.8 \\
\hline & Alle Methoden & 20.2 & 17.6 \\
\hline & Andere Methoden & 1.0 & 5.9 \\
\hline
\end{tabular}

Tabelle 24: Meinungen der Sportlehrkräfte der Mittelschule zur Methodik bei der sportartspezifischen Fertigkeitsvermittlung. 


\begin{tabular}{|l|l|c|c|}
\hline \multirow{2}{*}{ Item } & \multicolumn{1}{|c|}{ Antwortmöglichkeiten } & $\begin{array}{c}\text { Sportlehrer } \\
\text { Häufigkeiten in \% }\end{array}$ & $\begin{array}{c}\text { Sportlehrerinnen } \\
\text { Häufigkeiten in \% }\end{array}$ \\
\hline \hline \multirow{4}{*}{$\begin{array}{l}\text { 34 - Welche Lehrmetho- } \\
\text { den passen bei der Ver- } \\
\text { mittlung sportartspezifi- } \\
\text { scher Fertigkeiten zu Ihren } \\
\text { Schülern? }\end{array}$} & Nur Ganzheitsmethode & 1.9 & - \\
\cline { 2 - 4 } & Nur Teilmethode & 7.4 & - \\
\cline { 2 - 4 } & $\begin{array}{l}\text { Sur Ganz-Teil-Methode } \\
\text { auch Teilmethode }\end{array}$ & 9.3 & 18.2 \\
\cline { 2 - 4 } & $\begin{array}{l}\text { Sowohl Ganzheitsmethode als } \\
\text { auch Ganz-Teil-Methode }\end{array}$ & 29.6 & 45.5 \\
\cline { 2 - 4 } & $\begin{array}{l}\text { Sowohl Teilmethode als auch } \\
\text { Ganz-Teil-Methode }\end{array}$ & 11.1 & 9.1 \\
\cline { 2 - 4 } & Alle Methoden & 29.6 & 9.1 \\
\cline { 2 - 4 } & Andere Methoden & 1.9 & 18.2 \\
\hline
\end{tabular}

Tabelle 25: Meinungen der Sportlehrkräfte der Oberschule zur Methodik bei der sportartspezifischen Fertigkeitsvermittlung.

Die Tabellen 24 und 25 zur sportartspezifischen Fertigkeitsvermittlung im Hinblick auf die "großen Methoden“ der Sportdidaktik zeigen eine breitere Streuung, die schon durch das Antwortformat bedingt ist. Größere Bedeutung im Vergleich der Daten untereinander messen die Sportlehrkräfte jedoch tendenziell einer Mischung von Methoden zu. Ihrer Meinung nach sind v.a. die Ganz-Teil-Methode, sowohl Ganzheitsmethode als auch Teilmethode oder aber alle Methoden am Ehesten passend für die Schüler. Obwohl die gleichberechtigte Durchführung von Ganzheits- und Teilmethode von den meisten favorisiert wird (um 30\% der Sportlehrer und nahezu die Hälfte der Sportlehrerinnen), lässt sich den Daten kaum entnehmen, wie die Methoden in einzelnen Fällen kombiniert werden (wollen). Denn beispielsweise kommt die kombinierte Anwendung von Ganz-Teil-Methode mit anderen nur auf jeweils etwa 10\%. Daraus kann gefolgert werden, dass zwar eher eine Mischung von Methoden als wichtig bzw. passend für die Schüler betrachtet werden, jedoch eine ausdifferenzierte Anwendung kaum der Einstellung der Sportlehrkräfte entspricht. Die nur relativ geringe Zustimmung zur Ganz-TeilMethode lässt vermuten, dass eher wenig direkte Kombinationen bei der Vermittlung einzelner Fertigkeiten gewollt werden. Eine mögliche Lehreraussage wie „Bei manchen Fertigkeiten passt die Ganzheitsmethode und bei anderen die Teilmethode" könnte demnach den aus ihrer Sicht passende Methodencharakter beschreiben.

Die nun folgenden Tabellen geben einen weiteren Aspekt der allgemeinen Methodik wieder, nämlich das potenziell gewünschte individuelle Eingehen auf die Schüler. 


\begin{tabular}{|c|c|c|c|c|c|c|c|c|c|c|c|c|}
\hline \multirow{3}{*}{ Item } & \multicolumn{6}{|c|}{ Sportlehrer } & \multicolumn{6}{|c|}{ Sportlehrerinnen } \\
\hline & \multicolumn{5}{|c|}{ Häufigkeiten in \% } & \multirow{2}{*}{$\begin{array}{l}\text { Codierter } \\
\text { Mittelwert } \\
\end{array}$} & \multicolumn{5}{|c|}{ Häufigkeiten in \% } & \multirow{2}{*}{\begin{tabular}{|l} 
Codierter \\
Mitte/wert
\end{tabular}} \\
\hline & 1 & 2 & 3 & 4 & 5 & & 1 & 2 & 3 & 4 & 5 & \\
\hline $\begin{array}{l}35 \text { - In den überwie- } \\
\text { genden Situationen } \\
\text { sind individuelle } \\
\text { Lehrmethoden we- } \\
\text { sentlich. }\end{array}$ & 12.5 & 39.4 & 4.8 & 32.7 & 10.6 & 2.89 & 5.9 & 52.9 & 5.9 & 35.3 & - & 2.71 \\
\hline $\begin{array}{l}36 \text { - In den überwie- } \\
\text { genden Situationen } \\
\text { sind kollektive Lehr- } \\
\text { methoden wesentlich. }\end{array}$ & 38.5 & 32.7 & 19.2 & 7.7 & 1.9 & 2.02 & 41.2 & 23.5 & 23.5 & 11.8 & - & 2.06 \\
\hline
\end{tabular}

Tabelle 26: Meinungen der Sportlehrkräfte der Mittelschule zum individuellen vs. kollektiven Unterrichten.

\begin{tabular}{|c|c|c|c|c|c|c|c|c|c|c|c|c|}
\hline \multirow{3}{*}{ Item } & \multicolumn{6}{|c|}{ Sportlehrer } & \multicolumn{6}{|c|}{ Sportlehrerinnen } \\
\hline & \multicolumn{5}{|c|}{ Häufigkeiten in \% } & \multirow{2}{*}{$\begin{array}{c}\text { Codierter } \\
\text { Mittelwert }\end{array}$} & \multicolumn{5}{|c|}{ Häufigkeiten in \% } & \multirow{2}{*}{$\begin{array}{l}\text { Codierter } \\
\text { Mittelwer }\end{array}$} \\
\hline & 1 & 2 & 3 & 4 & 5 & & 1 & 2 & 3 & 4 & 5 & \\
\hline $\begin{array}{l}35 \text { - In den überwie- } \\
\text { genden Situationen } \\
\text { sind individuelle } \\
\text { Lehrmethoden we- } \\
\text { sentlich. }\end{array}$ & 18.5 & 25.9 & 7.4 & 42.6 & 5.6 & 2.91 & 9.1 & 45.5 & - & 45.5 & - & 2.82 \\
\hline $\begin{array}{l}36 \text { - In den überwie- } \\
\text { genden Situationen } \\
\text { sind kollektive Lehr- } \\
\text { methoden wesentlich. }\end{array}$ & 42.6 & 38.9 & 13.0 & 5.6 & - & 1.81 & 27.3 & 36.4 & 36.4 & - & - & 2.09 \\
\hline
\end{tabular}

Tabelle 27: Meinungen der Sportlehrkräfte der Oberschule zum individuellen vs. kollektiven Unterrichten.

Die Ergebnisse zum individuellen vs. kollektiven Unterrichten aus den Tabellen 26 und 27 zeigt ein weiteres Mal nur geringste Unterschiede zwischen Sportlehrerinnen und Sportlehrer sowie zwischen Mittel- und Oberschulen. Insgesamt stimmen die Sportlehrkräfte den kollektiven Methoden für ihren Sportunterricht zu und sind in Bezug auf individuelle Lehrmethoden durchschnittlich eher unentschieden. Letzteres bedeutet aber nicht, dass die meisten Sportlehrkräfte unentschieden sind, sondern sich diesbezüglich in zwei Lager teilen. Die eine Hälfte möchte in den überwiegenden Situationen individuell unterrichten und die andere Hälfte weist dies zurück. Unter Berücksichtigung der großen Klassen lässt sich folgende Vermutung anstellen: Die Einen weisen individuelle Methoden als unmöglich zurück und die anderen haben einen ausgeprägten Wunsch danach, der stärker ist als diese Bedingung - möglicherweise haben sie auch positive Erfahrungen mit individuellen Methoden gemacht bzw. gar Wege gefunden, diese anzuwenden. Ob- 
wohl etwa $10 \%$ der Mittelschullehrer umgekehrt kollektive Methoden sogar ablehnen, erhalten sie insgesamt die erwartete Zustimmung, was vor dem Hintergrund der großen Klassen als notwendige Einsicht zu werten ist. Da die individuellen Methoden aber nicht klar abgelehnt werden, lässt dies auf ein nicht unerhebliches Potenzial zu deren Implementierung schließen.

\section{Inhaltliche Schwerpunkte}

\begin{tabular}{|c|c|c|c|c|c|c|c|c|c|c|c|c|}
\hline \multirow{3}{*}{ Item } & \multicolumn{6}{|c|}{ Sportlehrer } & \multicolumn{6}{|c|}{ Sportlehrerinnen } \\
\hline & \multicolumn{5}{|c|}{ Häufigkeiten in \% } & \multirow{2}{*}{$\begin{array}{c}\text { Codierter } \\
\text { Mittelwert }\end{array}$} & \multicolumn{5}{|c|}{ Häufigkeiten in \% } & \multirow{2}{*}{$\begin{array}{l}\text { Codierter } \\
\text { Mittelwert }\end{array}$} \\
\hline & 1 & 2 & 3 & 4 & 5 & & 1 & 2 & 3 & 4 & 5 & \\
\hline $\begin{array}{l}37 \text { - Die Schüler sollen } \\
\text { vor allem Kraft ausbil- } \\
\text { den. }\end{array}$ & 44.2 & 51.0 & 3.8 & 1.0 & - & 1.62 & 64.7 & 29.4 & 5.9 & - & - & 1.41 \\
\hline $\begin{array}{l}38 \text { - Die Schüler sollen } \\
\text { vor allem Ausdauer } \\
\text { ausbilden. }\end{array}$ & 41.3 & 53.8 & 1.9 & 1.9 & 1.0 & 1.67 & 70.6 & 29.4 & - & - & - & 1.29 \\
\hline $\begin{array}{l}39 \text { - Die Schüler sollen } \\
\text { vor allem Beweg- } \\
\text { lichkeit erlangen. }\end{array}$ & 53.8 & 45.2 & 1.0 & - & - & 1.47 & 70.6 & 29.4 & - & - & - & 1.29 \\
\hline $\begin{array}{l}40 \text { - Die Schüler sollen } \\
\text { vor allem Schnelligkeit } \\
\text { erlangen. }\end{array}$ & 81.8 & 18.2 & - & - & - & 1.38 & 70.6 & 29.4 & - & - & - & 1.29 \\
\hline $\begin{array}{l}\text { 41-Die Schüler sollen } \\
\text { vor allem koordinative } \\
\text { Fähigkeiten ausbilden. }\end{array}$ & 62.5 & 34.6 & 1.0 & 1.0 & 1.0 & 1.43 & 88.2 & 11.8 & - & - & - & 1.12 \\
\hline $\begin{array}{l}42 \text { - Die Schüler sollen } \\
\text { vor allem motorische } \\
\text { Grundfertigkeiten } \\
\text { erwerben (wie Werfen, } \\
\text { Springen, Drehen, } \\
\text { Treffen). }\end{array}$ & 49.0 & 44.2 & 3.8 & 2.9 & - & 1.61 & 70.6 & 23.5 & - & 5.9 & - & 1.41 \\
\hline $\begin{array}{l}43 \text { - Die Schüler sollen } \\
\text { vor allem sportartspe- } \\
\text { zifische Fertigkeiten } \\
\text { erwerben (z.B. Kopf- } \\
\text { ball im Fußball, } \\
\text { Sprungwurf im Hand- } \\
\text { ball, Korbleger im } \\
\text { Basketball, Fosbury - } \\
\text { Flop). }\end{array}$ & 36.5 & 41.3 & 13.5 & 7.7 & 1.0 & 1.95 & 47.1 & 47.1 & - & 5.9 & - & 1.65 \\
\hline
\end{tabular}

Tabelle 28: Meinungen der Sportlehrkräfte der Mittelschule zu inhaltlichen Schwerpunkten des Sportunterrichts. 


\begin{tabular}{|c|c|c|c|c|c|c|c|c|c|c|c|c|}
\hline \multirow{3}{*}{ Item } & \multicolumn{6}{|c|}{ Sportlehrer } & \multicolumn{6}{|c|}{ Sportlehrerinnen } \\
\hline & \multicolumn{5}{|c|}{ Häufigkeiten in \% } & \multirow{2}{*}{$\begin{array}{c}\text { Codierter } \\
\text { Mittelwert }\end{array}$} & \multicolumn{5}{|c|}{ Häufigkeiten in \% } & \multirow{2}{*}{$\begin{array}{l}\text { Codierter } \\
\text { Mittelwert }\end{array}$} \\
\hline & 1 & 2 & 3 & 4 & 5 & & 1 & 2 & 3 & 4 & 5 & \\
\hline $\begin{array}{l}37 \text { - Die Schüler sollen } \\
\text { vor allem Kraft ausbil- } \\
\text { den. }\end{array}$ & 55.6 & 40.7 & 1.9 & 1.9 & - & 1.50 & 54.5 & 45.5 & - & - & - & 1.45 \\
\hline $\begin{array}{l}38 \text { - Die Schüler sollen } \\
\text { vor allem Ausdauer } \\
\text { ausbilden. }\end{array}$ & 50.0 & 44.4 & 3.7 & - & 1.9 & 1.59 & 45.5 & 54.5 & - & - & - & 1.55 \\
\hline $\begin{array}{l}39 \text { - Die Schüler sollen } \\
\text { vor allem Beweg- } \\
\text { lichkeit erlangen. }\end{array}$ & 64.8 & 29.6 & 3.7 & 1.9 & - & 1.43 & 81.8 & 18.2 & - & - & - & 1.18 \\
\hline $\begin{array}{l}40 \text { - Die Schüler sollen } \\
\text { vor allem Schnelligkeit } \\
\text { erlangen }\end{array}$ & 68.5 & 27.8 & 1.9 & 1.9 & - & 1.37 & 81.8 & 18.2 & - & - & - & 1.18 \\
\hline $\begin{array}{l}\text { 41-Die Schüler sollen } \\
\text { vor allem koordinative } \\
\text { Fähigkeiten ausbilden. }\end{array}$ & 70.4 & 25.9 & - & 3.7 & - & 1.37 & 72.7 & 27.3 & - & - & - & 1.27 \\
\hline $\begin{array}{l}42 \text { - Die Schüler sollen } \\
\text { vor allem motorische } \\
\text { Grundfertigkeiten } \\
\text { erwerben (wie Werfen, } \\
\text { Springen, Drehen, } \\
\text { Treffen). }\end{array}$ & 53.7 & 37.0 & - & 9.3 & - & 1.65 & 63.6 & 27.3 & 9.1 & - & - & 1.45 \\
\hline $\begin{array}{l}43 \text { - Die Schüler sollen } \\
\text { vor allem sportartspe- } \\
\text { zifische Fertigkeiten } \\
\text { erwerben (z.B. Kopf- } \\
\text { ball im Fußball, } \\
\text { Sprungwurf im Hand- } \\
\text { ball, Korbleger im } \\
\text { Basketball, Fosbury - } \\
\text { Flop). }\end{array}$ & 31.5 & 44.4 & 9.3 & 14.8 & - & 2.07 & 45.5 & 36.4 & 18.2 & - & - & 1.73 \\
\hline
\end{tabular}

Tabelle 29: Meinungen der Sportlehrkräfte der Oberschule zu inhaltlichen Schwerpunkten des Sportunterrichts.

Aus den Tabellen 28 und 29 wird ersichtlich, dass die breit gefächerten sportspezifischen Zielstellungen (Entwicklung und Verbesserung konditioneller Fähigkeiten und von Fertigkeiten) für die inhaltliche Ausgestaltung des Sportunterrichts insgesamt als sehr bedeutend von den Sportlehrkräften beurteilt werden. Erstmals sieht man nun einen durchgehenden Unterschied im Hinblick auf die Geschlechter, nicht aber im Hinblick auf Schulformen. Die Sportlehrerinnen geben den einzelnen Aspekten allen eine höhere Zustimmung als die Sportlehrer, auch wenn der Unterschied nur gering ist. In Bezug auf die konditionellen Aspekte zeigen Sportlehrer eine erkennbare Tendenz hin zu der Überzeugung, dass v.a. Schnelligkeit und Koordination ausgebildet werden soll. Die Sportlehrerinnen zeigen die gleiche Tendenz, zusätzlich aber noch eine hin zu Beweglichkeit. Bezüglich der Fertigkei- 
ten ist festzustellen, dass sie den konditionellen Fähigkeiten etwas nachstehen. Interessanterweise sind die Lehrkräfte beider Schulformen hier der Ansicht, dass stärker als die speziellen Techniken die motorischen Grundfertigkeiten ausgebildet werden sollten. Bei wie gesagt eher marginalen Unterschieden zwischen den Aspekten der inhaltlichen Schwerpunktsetzung kann also gefolgert werden, dass praktisch keine deutliche Bevorzugung einzelner Aspekte gewünscht wird, aber Inhalte der Gymnastik insgesamt doch etwas schwerer wiegen. Dies spiegelt ansatzweise den Lehrplan und etwas blasser die Entwicklung des ägyptischen Sportunterrichts wider.

\section{Bewertungsmethoden}

\begin{tabular}{|c|c|c|c|c|c|c|c|c|c|c|c|c|c|}
\hline \multirow{3}{*}{ Item } & \multirow{3}{*}{$\begin{array}{c}\text { Antwort- } \\
\text { möglichkeiten }\end{array}$} & \multicolumn{6}{|c|}{ Sportlehrer } & \multicolumn{6}{|c|}{ Sportlehrerinnen } \\
\hline & & \multicolumn{5}{|c|}{ Häufigkeiten in \% } & \multirow{2}{*}{$\begin{array}{c}\text { Codierter } \\
\text { Mittel- } \\
\text { wert }\end{array}$} & \multicolumn{5}{|c|}{ Häufigkeiten in \% } & \multirow{2}{*}{$\begin{array}{c}\text { Codierter } \\
\text { Mittel- } \\
\text { wert }\end{array}$} \\
\hline & & 1 & 2 & 3 & 4 & 5 & & 1 & 2 & 3 & 4 & 5 & \\
\hline $\begin{array}{l}44- \\
\text { Welche }\end{array}$ & $\begin{array}{l}\text { Regelmäßige } \\
\text { Bewertung }\end{array}$ & 33.7 & 1.9 & 6.7 & 6.7 & 1.0 & 1.89 & 35.3 & 58.8 & - & 5.9 & - & 1.76 \\
\hline $\begin{array}{l}\text { Bewer- } \\
\text { tungs- }\end{array}$ & $\begin{array}{l}\text { Zwischen- } \\
\text { bewertung }\end{array}$ & 14.4 & 55.8 & $\mid$\begin{tabular}{|l|}
15.4 \\
\end{tabular} & 5.8 & 8.7 & 2.38 & 17.6 & 52.9 & 11.8 & 11.8 & 5.9 & 2.35 \\
\hline $\begin{array}{l}\text { thoden } \\
\text { finden } \\
\text { Sie } \\
\text { wich- } \\
\text { tig? }\end{array}$ & Endbewertung & 53.8 & 30.8 & 7.7 & 6.7 & 1.0 & 1.70 & 76.5 & 5.9 & - & 11.8 & 5.9 & 1.65 \\
\hline
\end{tabular}

Tabelle 30: Meinungen der Sportlehrkräfte der Mittelschule zu allgemeinen Bewertungsmethoden.

\begin{tabular}{|c|c|c|c|c|c|c|c|c|c|c|c|c|c|}
\hline \multirow{3}{*}{ Item } & \multirow{3}{*}{$\begin{array}{l}\text { Antwort- } \\
\text { möglichkeiten }\end{array}$} & \multicolumn{6}{|c|}{ Sportlehrer } & \multicolumn{6}{|c|}{ Sportlehrerinnen } \\
\hline & & \multicolumn{5}{|c|}{ Häufigkeiten in \% } & \multirow{2}{*}{$\begin{array}{c}\text { Codierter } \\
\text { Mittel- } \\
\text { wert }\end{array}$} & \multicolumn{5}{|c|}{ Häufigkeiten in \% } & \multirow{2}{*}{$\begin{array}{c}\text { Codierter } \\
\text { Mittel- } \\
\text { wert }\end{array}$} \\
\hline & & 1 & 2 & 3 & 4 & 5 & & 1 & 2 & 3 & 4 & 5 & \\
\hline $\begin{array}{l}44- \\
\text { Welche }\end{array}$ & $\begin{array}{l}\text { Regelmäßig } \\
\text { Bewertung }\end{array}$ & 25.9 & 38.9 & 13.0 & 22.2 & - & 2.31 & 27.3 & 54.5 & 18.2 & & - & 1.91 \\
\hline $\begin{array}{l}\text { Bewer- } \\
\text { tungs- }\end{array}$ & $\begin{array}{l}\text { Zwischen } \\
\text { Bewertung }\end{array}$ & 24.1 & 42.6 & 24.1 & 3.7 & 5.6 & 2.24 & 27.3 & 36.4 & 18.2 & & 18.2 & 2.45 \\
\hline $\begin{array}{l}\text { meno- } \\
\text { den } \\
\text { finden } \\
\text { Sie } \\
\text { wich- } \\
\text { tig? }\end{array}$ & End Bewertung & 55.6 & 25.9 & 13.0 & 3.7 & 1.9 & 1.70 & 72.7 & 9.1 & 18.2 & 1 & - & 1.45 \\
\hline
\end{tabular}

Tabelle 31: Meinungen der Sportlehrkräfte der Oberschule zu allgemeinen Bewertungsmethoden. 
Die aus den Tabellen 30 und 31 hervorgehende Wichtigkeit von Bewertungsmethoden zeigt insgesamt eine hohe bis sehr hohe Gewichtung (männliche und weibliche Sportlehrer beider Schulformen). Die Sportlehrkräfte stimmen Bewertungsverfahren zu und haben ein Bewusstsein dafür. Jedoch zeigt sich im Hinblick auf regelmäßige und Zwischenbewertung eine breitere Streuung. Insbesondere die Zwischenbewertung zeigt in der Lehrermeinung eine deutliche Tendenz zur Mitte, worin eine Unentschlossenheit zum Ausdruck kommt. Daraus ist zu schließen, dass die Sportlehrkräfte den kontinuierlichen Einsatz von Bewertungsmethoden kaum verinnerlicht haben. Die folgenden Tabellen erfassen daher einen weiteren Aspekt der Bewertung, nämlich bestimmte Bewertungsmerkmale.

\begin{tabular}{|l|l|c|c|}
\hline \multirow{2}{*}{ Item } & \multirow{2}{*}{ Antwortmöglichkeiten } & Sportlehrer & Sportlehrerinnen \\
\cline { 3 - 4 } & & Häufigkeiten in \% & Häufigkeiten in \% \\
\hline \hline \multirow{2}{*}{$\begin{array}{l}\text { 45 - Welche Bewertungs- } \\
\text { inhalte sollten bei Prüfungen } \\
\text { berücksichtigt werden? }\end{array}$} & Beobachtungsmerkmale & 81.7 & 82.4 \\
\cline { 2 - 4 } & $\begin{array}{l}\text { Vorhergehende unterrichtsbe- } \\
\text { gleitende Notizen }\end{array}$ & 27.9 & 35.3 \\
\cline { 2 - 4 } & Gesundheitszustand & 18.3 & 17.6 \\
\cline { 2 - 4 } & Soziale Bedingungen & 3.0 & 23.5 \\
\hline
\end{tabular}

Tabelle 32: Meinungen der Sportlehrkräfte der Mittelschule zu Merkmalen einer Bewertung.

\begin{tabular}{|l|l|c|c|}
\hline \multirow{2}{*}{ Item } & \multirow{2}{*}{ Antwortmöglichkeiten } & Sportlehrer & Sportlehrerinnen \\
\cline { 3 - 4 } & & Häufigkeiten in \% & Häufigkeiten in \% \\
\hline \hline \multirow{2}{*}{$\begin{array}{l}45 \text { - Welche Bewertungs- } \\
\text { inhalte sollten bei Prüfungen } \\
\text { berücksichtigt werden? }\end{array}$} & Beobachtungsmerkmale & 83.3 & 81.8 \\
\cline { 2 - 4 } & $\begin{array}{l}\text { Vorhergehende unterrichts- } \\
\text { begleitende Notizen }\end{array}$ & 31.5 & 27.3 \\
\cline { 2 - 4 } & Gesundheitszustand & 20.4 & 9.0 \\
\cline { 2 - 4 } & Soziale Bedingungen & - & 6.0 \\
\hline
\end{tabular}

Tabelle 33: Meinungen der Sportlehrkräfte der Oberschule zu Merkmalen einer Bewertung.

Aus den Tabellen 32 und 33 geht hervor, dass die Sportlehrkräfte durchgehend das wesentliche Merkmal einer Bewertung als wichtig betrachten (je über $80 \%$ ) und knapp ein Drittel (dabei) Notizen als sinnvoll betrachtet. Interessant sind hier aber gewissermaßen zwei gewichtige „Ausreisser“: Die Sportlehrerinnen der Mittelschule wollen zu fast einem Viertel soziale Bedingungen berücksichtigen und die Sportlehrer beider Schulformen zu etwa 20\% den Gesundheitszustand der Schüler. Scheinbar ist durch die Ausbildung teilweise schon ein relativ ausdifferenziertes Bewusstsein für die Bewertung vorhanden. 


\subsubsection{Rahmenbedingungen}

An dieser Stelle seien die Ergebnisse zu notwendigen Rahmenbedingungen wiedergegeben (siehe dazu auch Kapitel 3.6). Die Hilfsmittel als notwendige bzw. gewünschte Aspekte des Sportunterrichts werden nicht erfasst, denn möglichst viele Hilfsmittel wünscht sich wohl jeder Lehrer.

\begin{tabular}{|c|c|c|c|c|c|c|c|c|c|c|c|c|c|}
\hline \multirow{3}{*}{ Item } & \multirow{3}{*}{$\begin{array}{c}\text { Antwort- } \\
\text { möglichkeiten }\end{array}$} & \multicolumn{6}{|c|}{ Sportlehrer } & \multicolumn{6}{|c|}{ Sportlehrerinnen } \\
\hline & & \multicolumn{5}{|c|}{ Häufigkeiten in \% } & \multirow{2}{*}{$\begin{array}{c}\text { Codierter } \\
\text { Mittel- } \\
\text { wert }\end{array}$} & \multicolumn{5}{|c|}{ Häufigkeiten in \% } & \multirow{2}{*}{$\begin{array}{c}\text { Codierte } \\
\text { Mittel- } \\
\text { wert }\end{array}$} \\
\hline & & 1 & 2 & 3 & 4 & 5 & & 1 & 2 & 3 & 4 & 5 & \\
\hline \multirow{6}{*}{$\begin{array}{l}\text { 46-Welche } \\
\text { Rahmen- } \\
\text { bedingun- } \\
\text { gen benö- } \\
\text { tigen Sie } \\
\text { unbedingt } \\
\text { für Ihren } \\
\text { Sportunter- } \\
\text { richt? }\end{array}$} & $\begin{array}{l}\text { Regelmäßige } \\
\text { Wartung der } \\
\text { Sportstätten und } \\
\text { der dazugehöri- } \\
\text { gen Einrichtun- } \\
\text { gen wie Gegens- } \\
\text { tände }\end{array}$ & 54.8 & 45.2 & - & - & - & 1.45 & 58.8 & 41.2 & - & - & - & 1.41 \\
\hline & $\begin{array}{l}\text { Adäquater Um- } \\
\text { kleideraum }\end{array}$ & 39.4 & 46.2 & 10.6 & 1.0 & 2.9 & 1.82 & 47.1 & 29.4 & 17.6 & 5.9 & - & 1.82 \\
\hline & $\begin{array}{l}\text { Ausreichende } \\
\text { Anzahl von } \\
\text { Toiletten und } \\
\text { Waschbecken }\end{array}$ & 60.6 & 38.5 & 1.0 & - & - & 1.40 & 82.4 & 17.6 & - & - & - & 1.18 \\
\hline & $\begin{array}{l}\text { Ärztliche Betreu- } \\
\text { ung bei Unfällen } \\
\text { der Schüler }\end{array}$ & 66.3 & 32.7 & 1.0 & - & - & 1.35 & 88.2 & 11.8 & - & - & - & 1.12 \\
\hline & $\begin{array}{l}\text { Eine Mahlzeit } \\
\text { pro Tag/Schüler }\end{array}$ & 64.4 & 24.0 & 8.7 & 1.9 & 1.0 & 1.51 & 82.4 & 17.6 & - & - & - & 1.18 \\
\hline & $\begin{array}{l}\text { Ein extra Bud- } \\
\text { get für den } \\
\text { Sportunterricht }\end{array}$ & 77.9 & 19.2 & 1.9 & - & 1.0 & 1.27 & 82.4 & 11.8 & 5.9 & - & - & 1.24 \\
\hline
\end{tabular}

Tabelle 34: Meinungen der Sportlehrkräfte der Mittelschule zu unbedingt benötigten Rahmenbedingungen. 


\begin{tabular}{|c|c|c|c|c|c|c|c|c|c|c|c|c|c|}
\hline \multirow{3}{*}{ Item } & \multirow{3}{*}{$\begin{array}{l}\text { Antwort- } \\
\text { möglichkeiten }\end{array}$} & \multicolumn{6}{|c|}{ Sportlehrer } & \multicolumn{6}{|c|}{ Sportlehrerinnen } \\
\hline & & \multicolumn{5}{|c|}{ Häufigkeiten in \% } & \multirow{2}{*}{$\begin{array}{c}\text { Codier- } \\
\text { ter } \\
\text { Mittel- } \\
\text { wert }\end{array}$} & \multicolumn{5}{|c|}{ Häufigkeiten in \% } & \multirow{2}{*}{$\begin{array}{c}\text { Codier- } \\
\text { ter } \\
\text { Mittel- } \\
\text { wert } \\
\end{array}$} \\
\hline & & 1 & 2 & 3 & 4 & 5 & & 1 & 2 & 3 & 4 & 5 & \\
\hline \multirow{6}{*}{$\begin{array}{l}\text { 46-Welche } \\
\text { Rahmenbe- } \\
\text { dingungen } \\
\text { benötigen } \\
\text { Sie unbe- } \\
\text { dingt für } \\
\text { Ihren Sport- } \\
\text { unterricht? }\end{array}$} & $\begin{array}{l}\text { Regelmäßige } \\
\text { Wartung der } \\
\text { Sportstätten und } \\
\text { der dazugehöri- } \\
\text { gen Einrichtun- } \\
\text { gen wie Gegens- } \\
\text { tände }\end{array}$ & 75.9 & 24.1 & - & - & - & 1.24 & 63.6 & 36.4 & - & - & - & 1.36 \\
\hline & $\begin{array}{l}\text { Adäquater Um- } \\
\text { kleideraum }\end{array}$ & 42.6 & 38.9 & 11.1 & 1.9 & 5.6 & 1.89 & 45.5 & 54.5 & - & & - & 1.55 \\
\hline & $\begin{array}{l}\text { Ausreichende } \\
\text { Anzahl von } \\
\text { Toiletten und } \\
\text { Waschbecken }\end{array}$ & 66.7 & 29.6 & - & 1.9 & 1.9 & 1.43 & 81.8 & 9.1 & - & - & 9.1 & 1.45 \\
\hline & $\begin{array}{l}\text { Ärztliche Betreu- } \\
\text { ung bei Unfällen } \\
\text { der Schüler }\end{array}$ & 68.5 & 29.6 & - & 1.9 & - & 1.35 & 90.9 & 9.1 & - & & - & 1.09 \\
\hline & $\begin{array}{l}\text { Eine Mahlzeit } \\
\text { pro Tag/Schüler }\end{array}$ & 72.2 & 20.4 & 5.6 & 1.9 & - & 1.37 & 72.7 & 9.1 & 18.2 & & - & 1.45 \\
\hline & $\begin{array}{l}\text { Ein extra Budget } \\
\text { für den Sportun- } \\
\text { terricht }\end{array}$ & 81.5 & 9.3 & 5.6 & 3.7 & - & 1.31 & 81.8 & 9.1 & 9.1 & - & - & 1.27 \\
\hline
\end{tabular}

Tabelle 35: Meinungen der Sportlehrkräfte der Oberschule zu unbedingt benötigten Rahmenbedingungen.

Erwartungsgemäß zeigt sich anhand einer Auswahl scheinbar grundsätzlich notwendiger Rahmenbedingungen für den Sportunterricht durch die Sportlehrkräfte eine hohe Zustimmung (Tabelle 34 und 35). Bei wiederum nur marginalen Unterschieden zwischen Schulformen und Geschlechtern ist jedoch ein Aspekt hervorstechend. Adäquate Umkleideräume sind zwar wichtig, jedoch nicht so bedeutsam wie Sportstätten selbst, sanitäre Anlagen, ärztliche Betreuung oder gar eine Mahlzeit/Tag für die Schüler und ein eigenes Budget. Für Sportlehrerinnen der Oberschule wird ein adäquater Umkleideraum jedoch wieder sehr bedeutsam. 


\subsubsection{Zusammenfassende Diskussion}

Zunächst ist festzustellen, dass sich insgesamt in Bezug auf Geschlecht und Schulform kaum Unterschiede in den Meinungen der Sportlehrkräfte herauskristallisierten. Lediglich punktuelle marginale Differenzen konnten aufgedeckt werden, auf die gleich eingegangen wird. Weiterhin zeigt sich in den drei erhobenen Kategorien Erziehungsziele, methodisches Vorgehen und Rahmenbedingungen insgesamt eine überaus deutliche Zustimmung. Vor dem Hintergrund der kulturellen Bedingungen ist diese starke Zustimmung -, die darauf schließen lässt, dass die einzelnen zerlegten Kategorien als Leitlinien verinnerlicht sind, - jedoch zu relativieren: Grundsätzlich gilt es in Ägypten als höflich, einer Aussage zunächst zuzustimmen. Kommt nun jemand von einem Sportinstitut und fragt nach den (letztlich dort gelehrten und) zu vermittelnden Zielen, Methoden und notwendigen Rahmenbedingungen, so werden sie folglich eher als wichtig beurteilt. Falls dies nicht den Tatsachen entsprechen sollte, wäre dies kaum überprüfbar. Besonders bedeutsam sind daher die Befunde, wo die Daten eine geringe Ablehnung zeigten; sie wiegen gewissermaßen schwerer als in den USA oder Deutschland. Die Ergebnisse seien nacheinander anhand der drei übergeordneten Kategorien resümiert und kommentiert.

Die Erziehungsziele des Sportunterrichts dienen als übergeordnete Leitlinien u.a, um ein vermehrtes Sporttreiben zu fördern (vgl. FARAG, 1999). Sie lassen sich nach den vorliegenden Ergebnissen nun in zwei Gruppen einteilen. Zur ersten Gruppe gehören psychische Eigenschaften, Gesundheit und Freude / Spaß / Begeisterung. Sie spiegelt in erster Linie den Charakter des Sporttreibens wider, der in Ägypten eher leistungsorientierter Wettkampfsport und gezieltes Training bedeutet. In dieser Gruppe waren kaum ablehnende Haltungen zu beobachten (mit übergreifenden Durchschnitten in den Unterkategorien von 1,2 - 1,4). Hervorzuheben sind hier lediglich die Befunde, dass die Sportlehrerinnen der Ausbildung von Führungsqualitäten ihrer Schülerinnen und ihre Vorbildfunktion geringer als ihre durchschnittlich etwas älteren männlichen Kollegen bewerten, jedoch stärker Hilfestellungen und Rückmeldungen geben wollen. Ursachen für die ersten beiden Ergebnisse lassen sich sicherlich in den kulturellen Bedingungen suchen, während Ursachen für die letzten beiden Ergebnisse noch offen bleiben müssen. 
Als zweite Gruppe lassen sich Freizeit, Sozialverhalten sowie sportspezifische Kenntnisse zusammenfassen. Sie weisen stärker auf Grundlagen eines selbständigen Sporttreibens hin. In dieser Gruppe tauchten immer wieder ablehnende Statements auf und so resultierten insgesamt höhere durchschnittliche Ergebnisse in den Unterkategorien $(1,6-1,85)$. Hierzu wurde bereits gefolgert, dass entweder eine situationsabhängige Beurteilung vorgenommen wurde oder aber Unsicherheiten bei den Sportlehrkräften bestehen. Möglicherweise wird den Erziehungszielen, die sich gewissermaßen wie von selbst bzw. nebenbei ergeben, auch per se geringere Bedeutungen zugeschrieben und sie sind in der Ausbildung „zu kurz gekommen“. Deutlich schwerer wiegt jedoch die Vermutung, dass die geringere Bewertung auf die faktische Bedingung der großen Klassen zurückzuführen ist. Hier erscheint es zudem häufig auch unmöglich, selbständiges Lernen zu kanalisieren - obwohl dies auch eindeutig gewünscht wird. Interessant ist in dieser zweiten Gruppe der Befund, dass v.a. bei den weiblichen Lehrkräften ablehnende Haltungen zu beobachten waren. Neben der Funktion des Sportunterrichts als Mittel der Förderung eines freizeitsportlichen Engagements (Schulsport) bezog sich dies auf selbständige Konfliktlösungen der Schüler(innen) und eigene Lernerfahrungen. Zwar ergaben sich wie gesagt meist deutliche Zustimmungen (und hier insbesondere bei sozial- und sportbezogenen Kenntnissen wie Sprache, Zusammenarbeit und Akzeptanz der Erfolge anderer sowie der Förderung des Schulsports), jedoch lassen sich die anderen aus pragmatischen Gründen bei über 40 SchülerInnen nur schwer umsetzen und dies stimmte wohl manche(n) um. Trotz übergreifender Zustimmung und trotz des ägyptischen Sportsystems besteht scheinbar gerade hier entsprechend Nachholbedarf für die Ausbildung.

Die Ergebnisse zum methodischen Vorgehen spiegeln diese Annahmen teilweise wider. Obwohl hier die Zustimmung ebenfalls hoch war (mit übergreifenden Durchschnittswerten von 1,4-2,4), ergab sich insbesondere im Hinblick auf Aspekte der sozialen Interaktion eine zwar zahlenmäßig gering ausgeprägte aber von der Bedeutung her nicht ungewichtige Ablehnungsquote. Dies zeigte sich insbesondere bei individuellen vs. kollektiven Methoden (mit dem höchsten Durchschnittswert von 2,4), wobei der Vorzug individueller Methoden zwar vielfach erkannt aber kaum umsetzbar scheint. 
Während die Wichtigkeit der allgemeinen Methoden Rückmeldung, Anleitung, Vormachen und Wettkämpfe ein weiteres Mal ein leistungssportliches Verständnis zum Vorschein kommen lassen (bei Sportlehrerinnen und Lehrkräften der Oberschule etwas stärker ausgeprägt), findet sich hier wiederum eine geringe Bedeutsamkeitsbewertung von selbständigem Handeln der Schüler (Prüfungsvorbereitung und Problemlösung) und Wettkämpfen in allen Sportarten, die bei den Sportlehrerinnen ausgeprägter ist. Zusammen mit obigen Befunden kann vermutet werden, dass sich die weiblichen (und etwas jüngeren) Sportlehrkräfte der Problematiken einerseits stärker bewusst sind, andererseits aber auch eher den gesellschaftlichen Konventionen folgen wollen.

Ähnliches gilt für die Inhaltlichen Schwerpunkte. Zwar wird die breite sportspezifische Zielstellung des Sportunterrichts erkannt (Zustimmung der Frauen wieder stärker!), jedoch sollen eher Grundfertigkeiten denn spezielle Fertigkeiten gelehrt werden. Da sich dies insbesondere auch in der Oberschule zeigt, kann gefolgert werden, dass es der Sportunterricht aus Grund- und Mittelschule bisher nicht verstanden hat, solche Grundfertigkeiten zu vermitteln. In kausaler Hinsicht ist hier ebenso auf die (methodische) Ausbildung zu verweisen oder aber auf die großen Klassen.

Weil in der ägyptischen Sportpädagogik häufig betont wird, dass es keine für jeden Fall geeignete Unterrichtsmethode gibt (vgl. z.B. ABD EL AZIZ, 1998), ist nicht verwunderlich, dass die Ergebnisse zur Methodik bei der sportartspezifischen Fertigkeitsvermittlung streuten. Einerseits wird "sowohl die Ganzheitsmethode als auch die Teilmethode" als Item von den SportlehrerInnen favorisiert, jedoch ist eine gezielte Kombination von Methoden - wie die Ergebnisse zur GanzTeil-Methode zeigen - andererseits kaum passend nach der Auffassung der Sportlehrkräfte. Vermutlich scheint hier nicht ganz klar, in welcher Vermittlungsart Kombinationen von Teilen und Ganzem effizient und gewinnbringend angewandt werden können.

Auch für Bewertungsmethoden (als Teil der Ausbildung) besteht ein Bewusstsein. Innen wird ein hoher Wert beigemessen, obwohl keine Noten im Zeugnis erscheinen - sie dienen lediglich zur Sicherung des Lernerfolges. In Bezug auf regelmäBige Bewertungen ist wiederum eine Unsicherheit festzustellen bzw. in Bezug auf Zwischenbewertungen eine erhebliche Ablehnung. Entweder scheuen die Sportlehrkräfte den zusätzlichen Aufwand oder aber die Auffassung YA'QUUTs (1992), 
ist weiter verbreitet als die von El HAMAHMI \& EL CHOULI (1990) oder RIDWAN (1997) (siehe S. 76). Bei der Bewertung sollten auch aus Sicht der Sportlehrerlnnen weiterhin die zentralen Bewegungsmerkmale herangezogen, allerdings werden Notizen nur von etwa einem Drittel als wichtig betrachtet. Dementsprechend scheint das Ziel von Bewertungen nicht sehr klar. Dafür spricht weiterhin die geringe und breit verteilte Bedeutsamkeitsbewertung gesundheitlicher (eher Sportlehrer) oder sozialer Aspekte (eher Sportlehrerinnen). Für die Ausbildung wäre entsprechend zu folgern, dass hier zielgenauere Methoden vermittelt werden sollten.

Bei nochmals geringen Unterschieden ist auch die Zustimmung im Hinblick auf erwünschte Rahmenbedingungen insgesamt groß (Durchschnitt 1,4). Hier ist jedoch davon auszugehen, dass sich nicht nur die Vorgaben des Lehrplans, sondern auch die Erfahrungen aus der Praxis deutlich auf dieses Meinungsbild niederschlagen. Einziger geschlechter-unterscheidender Befund liegt in adäquaten Umkleideräumen. Sportlehrerinnen sehen darin eine noch wichtigere Bedingung als Sportlehrer, womit die Neigung zu gesellschaftlicher Konformität wieder als bestätigt angesehen werden kann.

Betrachtet man die Befunde zu den einzelnen Kategorien im Hinblick auf Überschneidungen, so lassen sich zu den Meinungen der Sportlehrkräfte folgende Punkte resümieren:

1. Mittel- und Oberschullehrer unterscheiden sich praktisch nicht, jedoch orientieren sich Sportlehrerinnen etwas stärker als Sportlehrer an gesellschaftlichen Konventionen und erkennen Problemlagen scheinbar etwas deutlicher.

2. Erziehungsziele, die mit freudvollem Leistungssport/Trainieren verbunden sind, werden höher gewichtet.

3. Eine stärkere Berücksichtigung von Sozialverhalten im Sportunterricht und von spezifischen Methoden (sportartspezifische Vermittlung und Bewertung) für die Ausbildung scheint empfehlenswert. Dies betrifft neben Konfliktlösungen selbständiges Lernen und Handeln der SchülerInnen, die variantenreiche Anwendung der Ganz-Teil-Methode und den effektiven Umgang mit großen Klassen. 


\subsection{Praxis des Sportunterrichts}

Um die tabellarische Darstellungsform nicht übermäßig auszudehnen und weil sich bei der Meinung der Sportlehrkräfte in Bezug auf die beiden Schulformen kaum Unterschiede ergaben, werden die Items zur Praxis des Sportunterrichts nur noch in Einzelfällen anhand von zwei getrennten Tabellen (je Mittel- und Oberschule) dargestellt, aber die Tabellen wie zuvor kurz kommentiert. Auch die Analyse der Praxis des Sportunterrichts bzw. der tatsächlichen Umsetzung von Erziehungszielen, methodischem Vorgehen und dem Vorliegen verschiedener Rahmenbedingungen wie Hilfsmitteln folgt der groben Struktur aus Kapitel 5.1 (siehe auch Tab. 8, S. 91). Erinnert sei daran, dass am Ende der Unterkategorien eine zusammenfassende Beurteilung erfolgt.

\subsubsection{Erziehungsziele}

Die Implementierung von Erziehungszielen im Sportunterricht wurde bei der empirischen Prüfung an vielfachen Stellen durch Ergänzungsfragen abgesichert. Die häufigsten und hervorstechenden Antworten werden dann am Ende der jeweiligen Kommentare aufgenommen.

\section{Freizeit}

\begin{tabular}{|c|c|c|c|c|c|c|}
\hline Item & & & & & & \\
\hline \multirow{10}{*}{$\begin{array}{l}47 \text { - Lernen die Schüler, die Sportarten auch in } \\
\text { ihrer Freizeit selbständig, d.h. ohne Sie, auszu- } \\
\text { üben? }\end{array}$} & \multicolumn{6}{|c|}{ Mittelschule } \\
\hline & \multicolumn{3}{|c|}{ Sportlehrer } & \multicolumn{3}{|c|}{ Sportlehrerin } \\
\hline & \multicolumn{3}{|c|}{ Häufigkeiten in \% } & \multicolumn{3}{|c|}{ Häufigkeiten in \% } \\
\hline & $\mathrm{Ja}$ & Vielleicht & Nein & $\mathrm{Ja}$ & Vielleicht & Nein \\
\hline & 21.2 & 26.9 & 51.9 & 29.4 & 70.6 & - \\
\hline & \multicolumn{6}{|c|}{ Oberschule } \\
\hline & \multicolumn{3}{|c|}{ Sportlehrer } & \multicolumn{3}{|c|}{ Sportlehrerin } \\
\hline & \multicolumn{3}{|c|}{ Häufigkeiten in \% } & \multicolumn{3}{|c|}{ Häufigkeiten in \% } \\
\hline & $\mathrm{Ja}$ & Vielleicht & Nein & $\mathrm{Ja}$ & Vielleicht & Nein \\
\hline & 22.2 & 40.7 & 37.0 & 18.2 & 27.3 & 54.5 \\
\hline
\end{tabular}

Tabelle 36: Erlernen selbstständiger Ausführung der Sportarten. 
Aus den obigen Antworten der Sportlehrkräfte geht hervor, dass die Schüler kaum lernen, die unterrichteten Sportarten selbstständig in ihrer Freizeit auszuüben. Nur etwa $20 \%$ der Sportlehrkräfte sind der Ansicht, dass die Schüler die Sportarten auch eigenständig ausführen können. Beurteilt man die „Vielleicht-Aussagen“ vor dem Hintergrund einer möglichst objektiven Bewertung und gezielten Methodik durch die Sportlehrer, so rücken diese eher in die Nähe von „Nein“.

Etwa die Hälfte der Sportlehrkräfte antwortete hier nun mit „ja“ oder „vielleicht“. Die resultierende Kontrollfrage ergab, dass von ihnen als Lehrmaßnahme etwa 50\% eine freie Zeitverfügung im Sportunterricht zum Sporttreiben nach belieben nannten. Etwa ein Drittel orientiert sich an den Neigungen der Schüler bei ihren Empfehlungen für die Freizeit und argumentiert hinsichtlich der weitergehenden Aspekte Gesundheit und Sicherheit. Nur wenige Sportlehrkräfte sprechen talentierte Schüler an.

\begin{tabular}{|c|c|c|c|c|c|}
\hline \multirow{3}{*}{ Item } & \multirow{3}{*}{$\begin{array}{l}\text { Antwort- } \\
\text { möglichkeiten }\end{array}$} & \multicolumn{2}{|c|}{ Mittelschule } & \multicolumn{2}{|c|}{ Oberschule } \\
\hline & & Sportlehrer & $\begin{array}{c}\text { Sportlehre- } \\
\text { rinnen }\end{array}$ & Sportlehrer & $\begin{array}{c}\text { Sportlehre- } \\
\text { rinnen }\end{array}$ \\
\hline & & \multicolumn{2}{|c|}{ Häufigkeiten in \% } & \multicolumn{2}{|c|}{ Häufigkeiten in \% } \\
\hline \multirow{6}{*}{$\begin{array}{l}\text { 48- Bei welchen } \\
\text { Sportarten wei- } \\
\text { sen Sie auf Mög- } \\
\text { lichkeiten zur } \\
\text { Freizeitausübung } \\
\text { hin? }\end{array}$} & Fußball & $\begin{array}{l}79.8 \\
\end{array}$ & - & 94.4 & - \\
\hline & Handball & 12.5 & 76.5 & 20.4 & 27.3 \\
\hline & Basketball & 12.5 & 64.7 & 25.9 & 18.2 \\
\hline & Volleyball & 26.9 & 76.5 & 29.6 & 45.5 \\
\hline & Leichtathletik & 15.4 & 23.5 & 29.6 & 63.6 \\
\hline & Turnen & 13.3 & 11.8 & 16.6 & - \\
\hline
\end{tabular}

Tabelle 37: Hinweise auf die Möglichkeit zum Freizeitsport (nach Sportarten).

Tabelle 37 zeigt, dass die Sportlehrer am Häufigsten auf Möglichkeiten zur Freizeitausübung beim Fußball hinweisen (etwa 80-95\%), während die Sportlehrerinnen dies aber auch in einer mittleren Ausprägung tun. Umgekehrt findet dies am wenigsten beim Turnen statt. Während Leichtathletik in der Oberschule einen hohen Stellenwert in punkto Freizeit genießt, so ist dies in der Mittelschule eher Volleyball. Insgesamt weisen die Sportlehrerinnen der Mittelschule aber auf alle Spielsportarten hin. Bei den männlichen Lehrern gehen die Empfehlungen insgesamt so eindeutig in Richtung Fußball, dass die anderen Sportarten nicht so häufig empfohlen werden. Gymnastik taucht hier übrigens nicht auf, weil es keinen Schulsport Gymnastik gibt. 
Zusammenfassend ist festzustellen, dass das Erziehungsziel Freizeit von weniger als einem Viertel der Sportlehrkräfte umgesetzt wird. Diejenigen, die es aktiv verfolgen, lassen die Schüler hauptsächlich nach belieben Sport treiben und nur ein sehr geringer Teil sichtet Talente oder berücksichtigt gar individuelle Neigungen. Es ist zwar zu vermuten, dass auf Grundlage der Lehrerneigungen vielfach Empfehlungen erfolgen, jedoch dienen sie nicht als Lerngrundlage für selbständiges Sporttreiben, sondern als Einladung für den Schulsport. Dabei werden von den Männern v.a. Fußball, von den Frauen ein breiteres Spektrum an Spielsportarten und von den Oberschullehrern insbesondere Leichtathletik präferiert.

\section{Gesundheit}

\begin{tabular}{|c|c|c|c|c|c|c|}
\hline \multicolumn{7}{|l|}{ Item } \\
\hline \multirow{10}{*}{$\begin{array}{l}49 \text { - Lernen die Schüler etwas über den Zusam- } \\
\text { menhang von Bewegung und Gesundheit? }\end{array}$} & \multicolumn{6}{|c|}{ Mittelschule } \\
\hline & \multicolumn{3}{|c|}{ Sportlehrer } & \multicolumn{3}{|c|}{ Sportlehrerinnen } \\
\hline & & ufigkeiten & in $\%$ & Hä & ufigkeiten i & $n \%$ \\
\hline & $\mathrm{Ja}$ & Vielleicht & Nein & $\mathrm{Ja}$ & Vielleicht & Nein \\
\hline & 32.7 & 433.3 & 24.0 & 17.6 & 64.7 & 17.6 \\
\hline & \multicolumn{6}{|c|}{ Oberschule } \\
\hline & \multicolumn{3}{|c|}{ Sportlehrer } & \multirow{2}{*}{\multicolumn{3}{|c|}{ Sportlehrerinnen }} \\
\hline & \multicolumn{3}{|c|}{ Häufigkeiten in \% } & Häufigkeiten in \% & & \\
\hline & $\mathrm{Ja}$ & Vielleicht & Nein & $\mathrm{Ja}$ & Vielleicht & Nein \\
\hline & 27.8 & 48.1 & 24.15 & 18.2 & 18.2 & 63.6 \\
\hline
\end{tabular}

Tabelle 38: Erwerb gesundheitlicher Kenntnisse (Zusammenhang zwischen Bewegung und Gesundheit).

Bis auf die Antworten der Sportlehrerinnen der Oberschule zeigen die Ergebnisse zum ersten Aspekt des Erziehungsziels gesundheitlicher Kenntnisse eine annähernde Normalverteilung. Die meisten Sportlehrkräfte sind sich unsicher, ob ihre Schüler etwas darüber lernen. Nur um 20\% der Sportlehrerinnen und um 30\% der Sportlehrer antworteten mit einem eindeutigen ,ja“ und so kann gefolgert werden, dass in nur geringem Ausmaß gezielte Maßnahmen eingesetzt werden. Die häufigsten explizit genannten Maßnahmen waren bei der Kontrollfrage Hinweise zur „gesunden“ Übungsausführung bei der Gymnastik und die allgemeine Vermittlung von Kenntnissen als Theorie-Einheit, wenn die Praxis wegen Regen oder Wüstensturm nicht stattfinden kann. 


\begin{tabular}{|c|c|c|c|c|c|}
\hline \multirow{3}{*}{ Item } & \multirow{3}{*}{$\begin{array}{l}\text { Antwort- } \\
\text { möglichkeiten }\end{array}$} & \multicolumn{2}{|c|}{ Mittelschule } & \multicolumn{2}{|c|}{ Oberschule } \\
\hline & & Sportlehrer & $\begin{array}{l}\text { Sportlehre- } \\
\text { rinnen }\end{array}$ & Sportlehrer & $\begin{array}{l}\text { Sportlehre- } \\
\text { rinnen }\end{array}$ \\
\hline & & \multicolumn{2}{|c|}{ Häufigkeiten in \% } & \multicolumn{2}{|c|}{ Häufigkeiten in \% } \\
\hline \multirow{7}{*}{$\begin{array}{l}50 \text { - Bei welchen } \\
\text { Sportarten ver- } \\
\text { deutlichen Sie } \\
\text { solche Zusam- } \\
\text { menhänge? }\end{array}$} & Gymnastik & 63.6 & 59.4 & 41.8 & 53.1 \\
\hline & Fußball & 44.2 & - & 35.2 & - \\
\hline & Handball & 30.8 & 24.7 & 14.8 & 27.3 \\
\hline & Basketball & 37.5 & 47.1 & 16.7 & 27.3 \\
\hline & Volleyball & 29.8 & 22.9 & 18.5 & 27.3 \\
\hline & Leichtathletik & 40.4 & 13.4 & 44.4 & 27.3 \\
\hline & Turnen & 28.8 & 41.2 & 33.3 & 10.7 \\
\hline
\end{tabular}

Tabelle 39: Vermittlung gesundheitlicher Kenntnisse nach Sportarten (Zusammenhang zwischen Bewegung und Gesundheit).

Wie die Kontrollfrage zu Frage 49 schon gezeigt hat, werden erwartungsgemäß insbesondere im Rahmen der Gymnastik gesundheitliche Aspekte aufgegriffen, wobei dies in der Mittelschule ausgeprägter erscheint. Diese Tendenz zeigt sich aber auch bei den anderen Sportarten. Auffällig ist ebenso, dass den Sportlehrern dies bei der Leichtathletik in den Sinn kommt, aber auch Fußball Raum für gesundheitsrelevante Fragen bietet. Die relativ jungen Sportlehrerinnen der Mittelschule zeigen neben Gymnastik eine schwerpunktmäßige Vermittlung bei Basketball und Turnen und die Sportlehrerinnen der Oberschule bei Hand-, Basket- und Volleyball sowie Leichtathletik zu je knapp einem Drittel. Zu vermuten ist insgesamt, dass die Vermittlung gesundheitlicher Kenntnisse in Bezug auf Bewegung sich bei Gymnastik und Leichtathletik eher auf konditionelle Aspekte bezieht und in den anderen Sportarten eher Verletzungsprophylaxe gemeint ist. Mit Ausnahme der Gymnastik liegen die Werte jedoch unter $50 \%$.

\begin{tabular}{|c|c|c|c|c|c|c|}
\hline \multirow{11}{*}{$\begin{array}{l}51 \text { - Lernen die Schüler etwas über den Zusammen- } \\
\text { hang zwischen Ernährung und Gesundheit? }\end{array}$} & & & & & & \\
\hline & \multicolumn{6}{|c|}{ Mittelschule } \\
\hline & \multicolumn{3}{|c|}{ Sportlehrer } & \multicolumn{3}{|c|}{ Sportlehrerinnen } \\
\hline & \multicolumn{3}{|c|}{ Häufigkeiten in \% } & \multicolumn{3}{|c|}{ Häufigkeiten in \% } \\
\hline & $\mathrm{Ja}$ & Vielleicht & Nein & $\mathrm{Ja}$ & Vielleicht & Nein \\
\hline & 36.5 & 27.9 & 35.6 & 52.9 & 29.4 & 17.6 \\
\hline & \multicolumn{6}{|c|}{ Oberschule } \\
\hline & \multicolumn{3}{|c|}{ Sportlehrer } & \multicolumn{3}{|c|}{ Sportlehrerinnen } \\
\hline & \multicolumn{3}{|c|}{ Häufigkeiten in \% } & \multicolumn{3}{|c|}{ Häufigkeiten in \% } \\
\hline & $\mathrm{Ja}$ & Vielleicht & Nein & $\mathrm{Ja}$ & Vielleicht & Nein \\
\hline & 42.6 & 27.8 & 29.6 & 18.2 & 45.5 & 36.4 \\
\hline
\end{tabular}

Tabelle 40: Erwerb gesundheitlicher Kenntnisse (Zusammenhang zwischen Ernährung und Gesundheit). 
Die Tabelle 40 (Kenntnisse zum Zusammenhang von Ernährung und Gesundheit) zeigt ein diffuses Bild. Geschlechterunterschiede oder Unterschiede zwischen den Schulformen können aus den Daten nicht herausgelesen werden. Da die Kontrollantworten jedoch lediglich „allgemeine Informationen“ enthielten, ist zu folgern, dass dieses Unterrichtsziel nur geringfügig und wenn ja, nur unsystematisch aufgegriffen wird - manchmal ergibt sich dies wohl aus Gesprächen.

\begin{tabular}{|c|c|c|c|c|}
\hline Item & & & & \\
\hline \multirow{10}{*}{$\begin{array}{l}52 \text { - Vermitteln Sie Kenntnisse über das Herz- } \\
\text { Kreislauf-System? }\end{array}$} & \multicolumn{4}{|c|}{ Mittelschule } \\
\hline & \multirow{2}{*}{\multicolumn{2}{|c|}{$\begin{array}{l}\text { Sportlehrer } \\
\text { Häufigkeiten in \% }\end{array}$}} & \multirow{2}{*}{\multicolumn{2}{|c|}{$\begin{array}{c}\text { Sportlehrerinnen } \\
\text { Häufigkeiten in \% }\end{array}$}} \\
\hline & & & & \\
\hline & $\mathrm{Ja}$ & Nein & $\mathrm{Ja}$ & Nein \\
\hline & 24.0 & 76.0 & 17.6 & 82.4 \\
\hline & \multicolumn{4}{|c|}{ Oberschule } \\
\hline & \multicolumn{2}{|c|}{ Sportlehrer } & \multicolumn{2}{|c|}{ Sportlehrerinnen } \\
\hline & \multicolumn{2}{|c|}{ Häufigkeiten in \% } & \multicolumn{2}{|c|}{ Häufigkeiten in \% } \\
\hline & $\mathrm{Ja}$ & Nein & $\mathrm{Ja}$ & Nein \\
\hline & 42.6 & 57.4 & 18.2 & 81.8 \\
\hline
\end{tabular}

Tabelle 41: Vermittlung von Kenntnissen über das Herz-Kreislauf-System.

In den überwiegenden Fällen werden im Sportunterricht keine Kenntnisse über das Herz-Kreislaufsystem vermittelt. Dies zeigt sich für weibliche Lehrkräfte und Lehrkräfte der Mittelschule ausgeprägter. Der Befund ist insofern interessant, als die Aussagen zur Vermittlung von Zusammenhängen zwischen Bewegung und Gesundheit (Tabelle 39) relativiert werden müssen. Wenn Gymnastik und Leichtathletik sich an den konditionellen Fähigkeiten wie beispielsweise Ausdauer orientieren, so lässt dies eigentlich direkt auf das Herz-Kreislauf-System schließen. Da hier aber kaum explizites Wissen vermittelt wird, scheinen allgemeine Aussagen (wie auch schon bei Kenntnissen zur Ernährung) die Kenntnisvermittlung zu charakterisieren. Dies zeigte sich auch bei der Kontrollfrage nach den Maßnahmen. 


\begin{tabular}{|c|c|c|c|c|c|c|}
\hline Item & & & & & & \\
\hline \multirow{9}{*}{$\begin{array}{l}53 \text { - Lernen die Schüler die gesundheitliche Bedeu- } \\
\text { tung der Hygiene kennen? }\end{array}$} & \multicolumn{6}{|c|}{ Mittelschule } \\
\hline & \multicolumn{3}{|c|}{$\begin{array}{c}\text { Sportlehrer } \\
\text { Häufigkeiten in \% }\end{array}$} & \multicolumn{3}{|c|}{$\begin{array}{l}\text { Sportlehrerinnen } \\
\text { Häufigkeiten in \% }\end{array}$} \\
\hline & $\mathrm{Ja}$ & Vielleicht & Nein & $\mathrm{Ja}$ & Vielleicht & Nein \\
\hline & 72.1 & 14.4 & 13.5 & 58.8 & 29.4 & 11.8 \\
\hline & \multicolumn{6}{|c|}{ Oberschule } \\
\hline & \multicolumn{3}{|c|}{ Sportlehrer } & \multicolumn{3}{|c|}{ Sportlehrerinnen } \\
\hline & \multicolumn{3}{|c|}{ Häufigkeiten in \% } & \multicolumn{3}{|c|}{ Häufigkeiten in \% } \\
\hline & $\mathrm{Ja}$ & Vielleicht & Nein & $\mathrm{Ja}$ & Vielleicht & Nein \\
\hline & 51.9 & 16.7 & 31.5 & 9.1 & 9.1 & 81.8 \\
\hline
\end{tabular}

Tabelle 42: Vermittlung von Kenntnissen über Hygiene.

In der Mehrzahl der Fälle werden Kenntnisse über die gesundheitliche Bedeutung der Hygiene vermittelt. Nur die Sportlehrerinnen der Oberschule bilden eine Ausnahme und so ist insgesamt eine stärke Bedeutung in der Mittelschule festzustellen. Wichtigste genannte Maßnahme waren Hinweise zu einem sauberen Erscheinungsbild sowie die Anweisung, dass sich die Schüler nach dem Sport oder sonstigem Spiel waschen müssen.

Die Befunde zum Erziehungsziel gesundheitliche Kenntnisse lassen sich wie folgt zusammenfassen: Die Sportlehrkräfte sind sich oft nicht sicher, ob ihre Schüler etwas über die Gesundheit lernen. Hervorzuheben sind aber Maßnahmen, die durch die Weitergabe allgemeiner Informationen gekennzeichnet sind. Weil aber einerseits häufiger angegeben wird, dass in den einzelnen Sportarten Kenntnisse vermittelt werden, und andererseits kaum spezifische Informationen wie zum Herz-Kreislauf-System oder zu Ernährung genannt werden, kann gefolgert werden, dass das Erziehungsziel nicht in spezifischen Wissensbeständen mündet, sondern darunter in erster Linie Wissen um Verletzungsprophylaxe verstanden wird. Das Erziehungsziel Gesundheit wird zwar hoch bewertet („Trainingskonzept Gesundheit“), kommt in seiner Gänze in der Praxis also noch zu kurz. In Bezug auf Hygiene (Waschen nach dem Sport und Sauberkeit) werden aber wahrscheinlich schon in der Mittelschule Gewohnheiten ausgebildet. Schließlich ist zu erwähnen, dass die Sportlehrkräfte der Mittelschule das Erziehungsziel Gesundheit insgesamt eher verfolgen als die der Oberschule - aber eben eher in allgemeiner Art. 
Daher wird angenommen, dass in der Oberschule eine stärkere Konzentration auf sportartspezifische Inhalte erfolgt.

\section{Sozialverhalten}

\begin{tabular}{|l|c|c|c|c|}
\hline \multirow{2}{*}{ Item } & \multicolumn{2}{c|}{ Sportlehrer } & \multicolumn{2}{c|}{ Sportlehrerinnen } \\
\cline { 2 - 4 } & \multicolumn{2}{|c|}{ Häufigkeiten in \% } & \multicolumn{2}{c|}{ Häufigkeiten in \% } \\
\cline { 2 - 4 } & Ja & Nein & Ja & Nein \\
\hline \hline 54 - Lernen die Schüler ihre Konflikte selbst zu lösen? & 39.4 & 60.6 & 17.6 & 82.4 \\
\hline $\begin{array}{l}55 \text { - Vermitteln Sie den Schüler sprachliche Fähigkei- } \\
\text { ten zur Konfliktlösung? }\end{array}$ & 45.2 & 54.8 & 23.5 & 76.5 \\
\hline
\end{tabular}

Tabelle 43: Erlernen selbständiger Konfliktlösung (Mittelschule).

\begin{tabular}{|l|c|c|c|c|}
\hline \multirow{2}{*}{ Item } & \multicolumn{2}{c|}{ Sportlehrer } & \multicolumn{2}{c|}{ Sportlehrerinnen } \\
\cline { 2 - 4 } & Häufigkeiten in \% & \multicolumn{2}{c|}{ Häufigkeiten in \% } \\
\cline { 2 - 4 } & Ja & Nein & Ja & Nein \\
\hline \hline 54 - Lernen die Schüler ihre Konflikte selbst zu lösen? & 44.4 & 55.6 & - & 100.0 \\
\hline $\begin{array}{l}55 \text { - Vermitteln Sie den Schüler sprachliche Fähigkei- } \\
\text { ten zur Konfliktlösung? }\end{array}$ & 37.0 & 63.0 & 9.1 & 90.9 \\
\hline
\end{tabular}

Tabelle 44: Erlernen selbständiger Konfliktlösung (Oberschule).

Die eigenständige Konfliktlösung der Schüler sowie sprachliche Fähigkeiten dazu als Teile des Erziehungszieles Sozialverhalten werden in der überwiegenden Anzahl der Fälle nicht gelernt. Auffällig ist, dass dies deutlich stärker bei den Sportlehrerinnen und auch bei Sportlehrkräften der Oberschule der Fall ist. Bei den männlichen Lehrkräften geht die Konfliktlösung durch die Schüler jedoch zu einem größeren Teil in den Unterricht ein. Als wichtigste Maßnahmen der Protagonisten selbständiger Konfliktlösung wurden das explizite Thematisieren von Fehlverhalten (z.B. die Zurückweisung von Schimpfen durch den Islam), Ratschläge und die mögliche Vermeidung von Konflikten genannt. Ein geringerer Teil lässt in die Gespräche (während und nach den Unterrichtseinheiten) Themen wie Selbstvertrauen und Wertschätzung sowie Fragetechniken eingehen. Die tatsächliche Konfliktlösung bleibt aber immer noch dem Sportlehrer vorbehalten. 


\begin{tabular}{|c|c|c|c|c|c|c|}
\hline Item & & & & & & \\
\hline \multirow{10}{*}{$\begin{array}{l}56 \text { - Lernen die Schüler zusammen } \\
\text { zu arbeiten? }\end{array}$} & \multicolumn{6}{|c|}{ Mittelschule } \\
\hline & \multicolumn{3}{|c|}{ Sportlehrer } & \multicolumn{3}{|c|}{ Sportlehrerinnen } \\
\hline & \multicolumn{3}{|c|}{ Häufigkeiten in \% } & \multicolumn{3}{|c|}{ Häufigkeiten in \% } \\
\hline & $\mathrm{Ja}$ & Vielleicht & Nein & $\mathrm{Ja}$ & Vielleicht & Nein \\
\hline & 53.8 & 8.7 & 37.5 & 47.1 & 52.9 & - \\
\hline & \multicolumn{6}{|c|}{ Oberschule } \\
\hline & \multicolumn{3}{|c|}{ Sportlehrer } & \multicolumn{3}{|c|}{ Sportlehrerinnen } \\
\hline & \multicolumn{3}{|c|}{ Häufigkeiten in \% } & \multicolumn{3}{|c|}{ Häufigkeiten in \% } \\
\hline & $\mathrm{Ja}$ & Vielleicht & Nein & $\mathrm{Ja}$ & Vielleicht & Nein \\
\hline & 55.6 & 9.3 & 35.2 & 81.8 & 18.2 & - \\
\hline
\end{tabular}

Tabelle 45: Vermittlung von Zusammenarbeit.

Die deutliche Mehrheit der Sportlehrkräfte ist der Ansicht, dass ihre Schüler lernen, zusammen zu arbeiten. Dieses Votum fällt bei den Sportlehrerinnen wiederum klarer aus. Während hier keine eindeutige Verneinung viel, so ist dies bei den Sportlehrern zu annähernd 40\% der Fall. Obwohl die Zusammenarbeit meist gelernt wird, werden von den Sportlehrern wahrscheinlich mehr „Einzelkämpfer" ausgebildet.

Die Antworten auf die Kontrollfrage (Maßnahmen der Förderung) waren in ihrer Qualität vielfältig. Die meisten Sportlehrkräfte (70 bis $80 \%$ ) gaben an, dass ihre Schüler zusammen den Sportplatz und die Geräte für den Unterricht vorbereiten, Reihen- oder Kreisaufstellungen systematisch durchgeführt werden und bei Gruppeneinteilungen im Unterricht ein Schüler bzw. eine Schülerin das Kommando übernimmt. Nahezu die Hälfte der Lehrkräfte weist auf die Bedeutung von Hilfestellungen hin (z.B. beim Turnen) und noch ein Drittel verstand unter Zusammenarbeit das taktische Zusammenspiel in den Mannschaftssportarten.

\begin{tabular}{|c|c|c|c|c|c|}
\hline \multirow{3}{*}{ Item } & \multirow{3}{*}{$\begin{array}{l}\text { Antwort- } \\
\text { möglichkeiten }\end{array}$} & \multicolumn{2}{|c|}{ Mittelschule } & \multicolumn{2}{|c|}{ Oberschule } \\
\hline & & Sportlehrer & $\begin{array}{l}\text { Sportlehre- } \\
\text { rinnen }\end{array}$ & Sportlehrer & $\begin{array}{c}\text { Sportlehre- } \\
\text { rinnen }\end{array}$ \\
\hline & & \multicolumn{2}{|c|}{ Häufigkeiten in \% } & \multicolumn{2}{|c|}{ Häufigkeiten in \% } \\
\hline \multirow{7}{*}{$\begin{array}{l}57 \text { - Bei wel- } \\
\text { chen Sportar- } \\
\text { ten fördern } \\
\text { Sie die Zu- } \\
\text { samm- } \\
\text { enarbeit? }\end{array}$} & Gymnastik & 38.4 & 443.8 & 31.3 & 35.6 \\
\hline & Fußball & 75.0 & - & 81.5 & - \\
\hline & Handball & 43.3 & 82.4 & 31.5 & 36.4 \\
\hline & Basketball & 46.2 & 64.7 & 35.2 & 45.5 \\
\hline & Volleyball & 47.1 & 76.5 & 35.2 & 54.5 \\
\hline & Leichtathletik & 30.8 & 17.6 & 20.4 & 54.5 \\
\hline & Turnen & 12.8 & 11.8 & 11.1 & - \\
\hline
\end{tabular}

Tabelle 46: Vermittlung von Zusammenarbeit (nach Sportarten). 
Die Vermittlung von Zusammenarbeit in den einzelnen Sportarten scheint in den Mittelschulen etwas ausgeprägter. Insgesamt können die Befunde als Bestätigung von Ergebnissen aus der vorhergehenden Frage betrachtet werden. Reihen- und Kreisaufstellungen bei der Gymnastik und Zusammenspiel in den Mannschaftssportarten können hier klar als Unterrichtsinhalte erkannt werden. Darauf konzentrieren sich die Sportlehrkräfte, wobei die Hilfestellung im Turnen kaum verfolgt wird. Demgegenüber tritt Fußball bei den Sportlehrern wieder deutlich heraus, aber meist liegt die Berücksichtigung der Förderung der Zusammenarbeit unter $50 \%$, wenngleich die Mannschaftssportarten höhere Werte aufweisen.

\begin{tabular}{|c|c|c|c|c|c|c|}
\hline Item & & & & & & \\
\hline \multirow{10}{*}{$\begin{array}{l}58 \text { - Lernen die Schüler das Durchsetzen eines an- } \\
\text { deren zu akzeptieren? }\end{array}$} & \multicolumn{6}{|c|}{ Mittelschule } \\
\hline & \multirow{2}{*}{\multicolumn{3}{|c|}{$\begin{array}{l}\text { Sportlehrer } \\
\text { Häufigkeiten in \% }\end{array}$}} & \multirow{2}{*}{\multicolumn{3}{|c|}{$\begin{array}{c}\text { Sportlehrerinnen } \\
\text { Häufigkeiten in \% }\end{array}$}} \\
\hline & & & & & & \\
\hline & $\mathrm{Ja}$ & \begin{tabular}{|l} 
Vielleicht \\
\end{tabular} & Nein & $\mathrm{Ja}$ & \begin{tabular}{|l|} 
Vielleicht \\
\end{tabular} & Nein \\
\hline & 83.7 & 7.7 & 8.7 & 47.1 & 17.6 & 35.3 \\
\hline & \multicolumn{6}{|c|}{ Oberschule } \\
\hline & \multicolumn{3}{|c|}{ Sportlehrer } & \multicolumn{3}{|c|}{ Sportlehrerinnen } \\
\hline & \multicolumn{3}{|c|}{ Häufigkeiten in \% } & \multicolumn{3}{|c|}{ Häufigkeiten in \% } \\
\hline & $\mathrm{Ja}$ & Vielleicht & Nein & $\mathrm{Ja}$ & Vielleicht & Nein \\
\hline & 72.2 & 9.3 & 18.5 & 90.9 & - & 9.1 \\
\hline
\end{tabular}

Tabelle 47: Akzeptanz von Erfolgen anderer Schüler.

Die Sportlehrerinnen und Sportlehrer sind klar der Überzeugung, dass ihre Schülerinnen und Schüler das Durchsetzen anderer akzeptieren können. Die jüngeren Sportlehrerinnen der Mittelschule sind sich aber nicht so sicher. Bei den Maßnahmen beziehen die Sportlehrkräfte die Akzeptanz aber in erster Linie auf hervorragende Schüler; sie sollen akzeptiert werden und dies wird auch betont.

Zusammenfassend ergeben sich anhand der Ergebnisse zum Erziehungsziel Sozialverhalten anhand der Aspekten selbständige Konfliktlösung, Zusammenarbeit und Akzeptanz drei Kernpunkte: 1. Konflikte werden durch die Lehrer gelöst, wobei in Gesprächen für einen Umgang damit sensibilisiert wird. Bei Frauen und Lehrkräften der Oberschule ist dies etwas ausgeprägter. 2. Die Förderung der Zusammenarbeit geht in den Sportunterricht deutlich ein, wobei sie sich dies in der Hauptsache auf die Vorbereitung der Sportanlagen, bestimmte Aufstellungsformen (vornehmlich bei der Gymnastik) und das Zusammenspiel in den Mannschafts- 
sportarten bezieht. Dies lernen Schülerinnen wiederum ausgeprägter. 3. Die Schülerinnen und Schüler lernen v.a. hervorragende Klassenkameraden zu akzeptieren. Dies wird deutlich durch die Sportlehrkräfte gefördert.

\section{Psychische Eigenschaften}

\begin{tabular}{|c|c|c|c|c|c|c|}
\hline Item & & & & & & \\
\hline \multirow{10}{*}{$\begin{array}{l}59 \text { - Fördern Sie ge- } \\
\text { zielt das Selbstver- } \\
\text { trauen Ihrer Schüler? }\end{array}$} & \multicolumn{6}{|c|}{ Mittelschule } \\
\hline & \multicolumn{3}{|c|}{ Sportlehrer } & \multicolumn{3}{|c|}{ Sportlehrerinnen } \\
\hline & \multicolumn{3}{|c|}{ Häufigkeiten in \% } & \multicolumn{3}{|c|}{ Häufigkeiten in \% } \\
\hline & Ja & Nein & $\begin{array}{c}\text { Das Selbstvertrauen } \\
\text { wird automatisch } \\
\text { gestärkt }\end{array}$ & Ja & Nein & $\begin{array}{c}\text { Das Selbstvertrauen } \\
\text { wird automatisch } \\
\text { gestärkt }\end{array}$ \\
\hline & 61.5 & 4.8 & 33.7 & 76.5 & 5.9 & 17.6 \\
\hline & \multicolumn{6}{|c|}{ Oberschule } \\
\hline & \multicolumn{3}{|c|}{ Sportlehrer } & \multicolumn{3}{|c|}{ Sportlehrerinnen } \\
\hline & \multicolumn{3}{|c|}{ Häufigkeiten in \% } & \multicolumn{3}{|c|}{ Häufigkeiten in \% } \\
\hline & Ja & Nein & $\begin{array}{c}\text { Das Selbstvertrauen } \\
\text { wird automatisch } \\
\text { gestärkt }\end{array}$ & Ja & Nein & $\begin{array}{c}\text { Das Selbstvertrauen } \\
\text { wird automatisch } \\
\text { gestärkt }\end{array}$ \\
\hline & 68.5 & 3.7 & 27.8 & 45.5 & 18.2 & 36.4 \\
\hline
\end{tabular}

Tabelle 48: Förderung des Selbstvertrauens.

Die Mehrheit der Lehrer ist der Meinung, dass sie das Selbstvertrauen ihrer Schüler fördern und nur ein geringer Teil tut dies nicht. Bemerkenswert sind aber auch die insgesamt knapp 30\%, die davon ausgehen, dass das Selbstvertrauen automatisch durch den Sportunterricht bzw. das Sporttreiben gefördert wird. Es kann also davon gesprochen werden, dass die Sportlehrkräfte in Ägypten meist klar das Erziehungsziel Förderung des Selbstvertrauens verfolgen, dies aber auch häufiger dem Zufall überlassen. 


\begin{tabular}{|c|c|c|c|c|}
\hline Item & \multicolumn{4}{|c|}{ Mittelschule } \\
\hline \hline \multirow{4}{*}{60 - Erwerben die Schüler „Kampfgeist“? } & \multicolumn{4}{|c|}{ Sportlehrerinnen } \\
\cline { 2 - 5 } & \multicolumn{2}{|c|}{ Sportlehrer } & \multicolumn{2}{|c|}{ Späufigkeiten in \% } \\
\cline { 2 - 5 } & Häufigkeiten in \% & Ja & Nein \\
\cline { 2 - 5 } & Ja & Nein & Ja & \multicolumn{4}{|c|}{ Oberschule } \\
\cline { 2 - 5 } & 81.7 & 18.3 & 88.2 & 11.8 \\
\cline { 2 - 5 } & \multicolumn{3}{|c|}{ Sportlehrer } & \multicolumn{2}{|c|}{ Sportlehrerinnen } \\
\cline { 2 - 5 } & Häufigkeiten in \% & Häufigkeiten in \% \\
\cline { 2 - 5 } & Ja & Nein & Ja & Nein \\
\cline { 2 - 5 } & 64.8 & 35.2 & 54.5 & 45.5 \\
\hline
\end{tabular}

Tabelle 49: Erwerb von „Kampfgeist“.

Der überwiegende Teil der SportlehrerInnen ist der Meinung, dass ihre Schützlinge „Kampfgeist“ erwerben. Interessanterweise ist dies wieder in den Mittelschulen ausgeprägter. Vor dem Hintergrund des Schulsports wird als konkrete Maßnahmen am Häufigsten die Durchführung von Wettkämpfen im Sportunterricht genannt. Zu einem geringeren Teil werden die Schüler weiterhin von den Lehrern ermutigt, Schwierigkeiten zu überwinden und es wird meist betont, dass sie gewinnen sollen.

\begin{tabular}{|l|c|c|c|c|c|c|}
\hline \multirow{2}{*}{ Item } & \multicolumn{3}{|c|}{ Sportlehrer } & \multicolumn{3}{c|}{ Sportlehrerinnen } \\
\cline { 2 - 7 } & \multicolumn{3}{|c|}{ Häufigkeiten in \% } & \multicolumn{3}{c|}{ Häufigkeiten in \% } \\
\cline { 2 - 7 } & Ja & Vielleicht & Nein & Ja & Vielleicht & Nein \\
\hline \hline $\begin{array}{l}\text { 61 - Erwerben die Schüler Führungsqualitä- } \\
\text { ten? }\end{array}$ & 74.0 & 16.3 & 9.6 & 100.0 & - & - \\
\hline $\begin{array}{l}\text { 62 - Lernen die Schüler Gehorsam bei Anwei- } \\
\text { sungen und Befehlen? }\end{array}$ & 83.7 & 1.9 & 14.4 & 82.4 & - & 17.6 \\
\hline 63 - Lernen die Schüler, sich zu bemühen? & 54.8 & 26.0 & 19.2 & 52.9 & 35.3 & 11.8 \\
\hline
\end{tabular}

Tabelle 50: Erwerb weiterer psychischer Eigenschaften (Mittelschule).

\begin{tabular}{|l|c|c|c|c|c|c|}
\hline \multirow{2}{*}{ Item } & \multicolumn{3}{|c|}{ Sportlehrer } & \multicolumn{3}{c|}{ Sportlehrerinnen } \\
\cline { 2 - 7 } & \multicolumn{3}{|c|}{ Häufigkeiten in \% } & \multicolumn{2}{c|}{ Häufigkeiten in \% } \\
\cline { 2 - 7 } & Ja & Vielleicht & Nein & Ja & Vielleicht & Nein \\
\hline \hline $\begin{array}{l}\text { 61 - Erwerben die Schüler Führungsqualitä- } \\
\text { ten? }\end{array}$ & 64.8 & 25.9 & 9.3 & 100.0 & - & - \\
\hline $\begin{array}{l}\text { 62 - Lernen die Schüler Gehorsam bei Anwei- } \\
\text { sungen und Befehlen? }\end{array}$ & 88.9 & - & 11.1 & 90.9 & - & 9.1 \\
\hline 63 - Lernen die Schüler, sich zu bemühen? & 53.7 & 27.8 & 18.5 & 45.5 & 9.1 & 45.5 \\
\hline
\end{tabular}

Tabelle 51: Erwerb weiterer psychischer Eigenschaften (Oberschule). 
Die überwiegende Mehrheit der Sportlehrkräfte bejaht, dass ihre Schüler Führungsqualitäten erwerben - die Lehrerinnen sogar vollkommen. Eine ähnlich starke Zustimmung erfährt das Lernen von Gehorsam. Schwächer, aber immer noch positiv, sind die Einschätzungen zum eigenen Bemühen der Schüler.

Interessant sind nun die konkreten Maßnahmen. Während die Sportlehrkräfte zur Führungsqualität zu etwa zwei Drittel angeben, dass ihre Schüler immer Anleitungen erhalten und das Richtige vs. Falsche erklärt wird, sowie knapp die Hälfte angibt, dass beim Erbringen von Leistung auch die Gesundheit zu berücksichtigen ist, zeigt sich hier ein bestimmtes Bild von Führungsqualität. Hier wird auf das Befolgen von Normen abgehoben, zu denen auch ein gesunder Körper gehört. Dies zeigt sich ebenfalls im Hinblick auf Gehorsam, wobei über 70\% der Sportlehrkräfte zwei wichtige Maßnahmen angeben: a) Die Vor- und Nachbereitung der Sportstätten (siehe dazu auch die Ergebnisse zum Item 56: Zusammenarbeit); b) Die Bestrafung ungehorsamer Schüler bis zum Verweis aus dem Unterricht. Die Schüler sollen also insgesamt also gewissermaßen alle „spuren“. Etwas gegenläufig sind daher auch möglicherweise die Angaben zum eigenen Bemühen der Schüler, wobei hier wieder relativ unkonkrete Maßnahmen wie Durchführung von Wettkämpfen im Unterricht mit dem Hinweis auf Anstrengung und in geringerem Maße Gespräche mit Schülern angegeben werden.

Im Hinblick auf das Erziehungsziel psychischer Eigenschaften ist zusammenfassend festzustellen, dass ein weiteres Mal keine systematischen Geschlechtsunterschiede und keine Unterschiede zwischen den Schulformen ausmachen lassen. Die Schülerinnen und Schüler erwerben aus Sicht der Sportlehrkräfte Selbstvertrauen (zu häufig allerdings eher zufällig), „Kampfgeist“ (auch hier meist wenig gezielt durch konkrete Wettkampfformen und Zuspruch) und werden zur Konformität erzogen. Diese Aspekte sind insgesamt als gute Bedingungen im Hinblick auf den Schul- bzw. Leistungssport anzusehen. Ein vorgegebenes Trainingsprogramm kann mit solchen Schülern problemlos durchgeführt werden. Allerdings ist fraglich, ob dadurch tatsächlich weitergehende Erfolge erzielt werden können, denn in Belastungssituationen können wenig konkret erworbenes Selbstvertrauen und „Kampfgeist“ kaum als gesicherte Grundlage für Erfolg dienen, d.h. die Schüler können in solchen Situationen eher „scheitern“. 


\begin{tabular}{|l|c|c|c|c|c|c|}
\hline \multirow{2}{*}{ Item } & \multicolumn{3}{|c|}{ Sportlehrer } & \multicolumn{3}{c|}{ Sportlehrerinnen } \\
\cline { 2 - 7 } & \multicolumn{2}{|c|}{ Häufigkeiten in \% } & \multicolumn{2}{c|}{ Häufigkeiten in \% } \\
\cline { 2 - 7 } & Ja & Vielleicht & Nein & Ja & Vielleicht & Nein \\
\hline \hline $\begin{array}{l}\text { 64 - Lernen die Schüler die räumlichen und/oder } \\
\text { gerätebezogenen Regeln bei den unterschiedli- } \\
\text { chen Sportarten? }\end{array}$ & 23.1 & 25.0 & 51.9 & 76.5 & 17.6 & 5.9 \\
\hline $\begin{array}{l}\text { 65-Lernen die Schüler die Einhaltung von Spiel- } \\
\text { regeln und von taktischen Regeln? }\end{array}$ & 18.3 & 16.3 & 65.4 & 64.7 & 17.6 & 17.6 \\
\hline $\begin{array}{l}\text { 66 - Berichten Sie den Schüler über wichtige } \\
\text { aktuelle Sportereignisse (z.B. Fußballweltmeis- } \\
\text { terschaft, Olympische Spiele)? }\end{array}$ & 20.2 & 26.9 & 52.9 & 35.3 & 52.9 & 11.8 \\
\hline
\end{tabular}

Tabelle 52: Erwerb sportspezifischer Kenntnisse (Mittelschule).

\begin{tabular}{|l|c|c|c|c|c|c|}
\hline \multirow{2}{*}{ Item } & \multicolumn{3}{c|}{ Sportlehrer } & \multicolumn{3}{c|}{ Sportlehrerinnen } \\
\cline { 2 - 7 } & \multicolumn{2}{|c|}{ Häufigkeiten in \% } & \multicolumn{2}{c|}{ Häufigkeiten in \% } \\
\cline { 2 - 7 } & Ja & Vielleicht & Nein & Ja & Vielleicht & Nein \\
\hline $\begin{array}{l}\text { 64 - Lernen die Schüler die räumlichen und/oder } \\
\text { gerätebezogenen Regeln bei den unterschiedli- } \\
\text { chen Sportarten? }\end{array}$ & 38.9 & 24.1 & 37.0 & 72.7 & - & 27.3 \\
\hline $\begin{array}{l}\text { 65-Lernen die Schüler die Einhaltung von Spiel- } \\
\text { regeln und von taktischen Regeln? }\end{array}$ & 22.2 & 22.2 & 55.6 & 9.1 & 9.1 & 81.8 \\
\hline $\begin{array}{l}\text { 66 - Berichten Sie den Schüler über wichtige } \\
\text { aktuelle Sportereignisse (z.B. Fußballweltmeis- } \\
\text { terschaft, Olympische Spiele)? }\end{array}$ & 24.1 & 25.9 & 50.0 & 27.3 & 18.2 & 54.5 \\
\hline
\end{tabular}

Tabelle 53: Erwerb sportspezifischer Kenntnisse (Oberschule).

Überraschenderweise verneint ein Großteil der Lehrer den Erwerb sportspezifischer Kenntnisse, wobei die Aspekte insgesamt von den jüngeren Sportlehrerinnen der Mittelschule und die Regelkenntnisse (der Regelwerke) in der Oberschule bejaht werden. In Bezug auf Letzteres zeigt sich damit insgesamt ein positives Verhältnis. Die meisten Lehrer stellen zusammen genommen kaum Bezüge zum aktuellen Sportgeschehen her (was den Unterricht lebendiger und spannender machen könnte), unterrichten kaum taktische Regeln, bringen den Schülern aber meist die grundlegenden Regeln der Sportarten bei.

Als konkrete Maßnahmen nennen die Sportlehrkräfte, die die Items bejahen, nur zu einem geringen Teil, dass Regeln während des Sporttreibens thematisiert werden und sie schiedsen. Wenige nennen allgemeine Informationen über Taktiken und Spielabläufe und Einige weisen auf aktuelle Wettkampftermine aktueller Sportereignisse hin. 


\begin{tabular}{|c|c|c|c|c|}
\hline Item & & & & \\
\hline \multirow{10}{*}{$\begin{array}{l}67 \text { - Gestalten Sie Ihren Sportunterricht so, dass } \\
\text { die Schüler möglichst viele eigene Lernerfahrun- } \\
\text { gen sammeln? }\end{array}$} & \multicolumn{4}{|c|}{ Mittelschule } \\
\hline & \multirow{2}{*}{\multicolumn{2}{|c|}{$\begin{array}{l}\text { Sportlehrer } \\
\text { Häufigkeiten in \% }\end{array}$}} & \multicolumn{2}{|c|}{ Sportlehrerinnen } \\
\hline & & & \multicolumn{2}{|c|}{ Häufigkeiten in \% } \\
\hline & Ja Nein & Nein & $\mathrm{Ja}$ & Nein \\
\hline & 22.1 & 77.9 & 58.8 & 41.2 \\
\hline & \multicolumn{4}{|c|}{ Oberschule } \\
\hline & \multicolumn{2}{|c|}{ Sportlehrer } & \multicolumn{2}{|c|}{ Sportlehrerinnen } \\
\hline & \multicolumn{2}{|c|}{ Häufigkeiten in \% } & \multicolumn{2}{|c|}{ Häufigkeiten in \% } \\
\hline & $\mathrm{Ja}$ & Nein & $\mathrm{Ja}$ & Nein \\
\hline & 18.5 & 81.5 & 27.3 & 72.7 \\
\hline
\end{tabular}

Tabelle 54: Vermittlung vielfältiger Lernerfahrungen.

Außer von den Sportlehrerinnen der Mittelschule wird deutlich verneint, dass ihr Unterricht möglichst viele selbständige Lernerfahrungen ermöglicht. Dies bestätigt die vorhergehenden Befunde, die in Richtung Konformität weisen (Sozialverhalten und psychische Eigenschaften). Auch die Kontrollfrage der etwa 20\% Befürworter selbständiger Lernerfahrungen zeigt, dass mit Kenntnisvermittlung zu möglichst allen Aspekten von Wettkämpfen kaum Raum für eigene Lerninhalte gegeben scheint.

Zusammenfassend ergeben sich zum Erziehungsziel sportspezifische Kenntnisse folgende Kernpunkte: Zu einem gewichtigen Anteil werden diese zentralen Aspekte des Sporttreibens nicht gelehrt. Wenngleich die Regeln der Sportarten meist vermittelt werden (denn ohne sie kann die Durchführung kaum gelingen), kommen taktische Inhalte, Informationen zu Sportereignissen und selbständige Lernerfahrungen deutlich zu kurz. Unterschiede zwischen Geschlechtern und Schulformen sind nur punktuell zu beobachten. 


\section{Freude/Spaß/Begeisterung}

\begin{tabular}{|c|c|c|c|c|}
\hline Item & & & & \\
\hline \multirow{10}{*}{$\begin{array}{l}68 \text { - Geben Sie möglichst viele positive Rückmel- } \\
\text { dungen, um die Schüler stolz auf ihre sportlichen } \\
\text { Handlungen zu machen? }\end{array}$} & \multicolumn{4}{|c|}{ Mittelschule } \\
\hline & \multirow{2}{*}{\multicolumn{2}{|c|}{$\begin{array}{c}\text { Sportlehrer } \\
\text { Häufigkeiten in \% }\end{array}$}} & \multirow{2}{*}{\multicolumn{2}{|c|}{$\begin{array}{c}\text { Sportlehrerinnen } \\
\text { Häufiakeiten in \% }\end{array}$}} \\
\hline & & & & \\
\hline & $\mathrm{Ja}$ & Nein & $\mathrm{Ja}$ & Nein \\
\hline & 92.3 & 7.7 & 94.1 & 5.9 \\
\hline & \multicolumn{4}{|c|}{ Oberschule } \\
\hline & \multirow{2}{*}{\multicolumn{2}{|c|}{$\begin{array}{c}\text { Sportlehrer } \\
\text { Häufigkeiten in \% }\end{array}$}} & \multirow{2}{*}{\multicolumn{2}{|c|}{$\begin{array}{c}\text { Sportlehrerinnen } \\
\text { Häufigkeiten in \% }\end{array}$}} \\
\hline & & & & \\
\hline & $\mathrm{Ja}$ & Nein & $\mathrm{Ja}$ & Nein \\
\hline & 87.0 & 13.0 & 100.0 & - \\
\hline
\end{tabular}

Tabelle 55: Positive Rückmeldungen durch die Sportlehrkräfte.

Die Sportlehrkräfte Ägyptens geben ein klares Votum zu positiven Rückmeldungen ab, denn nahezu alle bemühen sich in ihrem Unterricht dazu. Als konkrete Maßnahmen nannte je etwa die Hälfte, dass sie die Schüler zu guten Leistungen immer wieder ermuntern und erfolgreiche Schüler vor der ganzen Klasse loben. Ob allerdings ausgeprägtes Feedback bei der sportartspezifischen Fertigkeitsvermittlung gegeben wird, bleibt fraglich. Zu vermuten ist jedoch, dass zumindest gute Schüler durch häufiges Herausheben aus der Gruppe Freude oder Begeisterung entwickeln.

\begin{tabular}{|c|c|c|c|c|c|c|c|c|c|c|}
\hline Item & & & & & & & & & & \\
\hline \multirow{10}{*}{$\begin{array}{l}\text { 69- Bieten } \\
\text { Sie Hilfe } \\
\text { an? }\end{array}$} & \multicolumn{10}{|c|}{ Mittelschule } \\
\hline & \multicolumn{5}{|c|}{ Sportlehrer } & \multicolumn{5}{|c|}{ Sportlehrerinnen } \\
\hline & \multicolumn{4}{|c|}{ Häufigkeiten in \% } & \multirow[b]{2}{*}{$\begin{array}{l}\text { Codierter } \\
\text { Mittelwert }\end{array}$} & \multicolumn{4}{|c|}{ Häufigkeiten in \% } & \multirow{2}{*}{$\begin{array}{l}\text { Codierter } \\
\text { Mittelwert }\end{array}$} \\
\hline & Immer & Meistens & $\begin{array}{c}\text { Manch } \\
\text { mal }\end{array}$ & $\mathrm{Nie}$ & & Immer & Meistens & $\begin{array}{c}\text { Manch } \\
\text { mal }\end{array}$ & $\mathrm{Nie}$ & \\
\hline & 30.8 & 36.5 & 24.0 & 8.7 & 2.11 & 17.6 & 5.9 & 76.5 & - & 2.59 \\
\hline & \multicolumn{10}{|c|}{ Oberschule } \\
\hline & \multicolumn{5}{|c|}{ Sportlehrer } & \multicolumn{5}{|c|}{ Sportlehrerinnen } \\
\hline & \multicolumn{4}{|c|}{ Häufigkeiten in \% } & \multirow[b]{2}{*}{$\begin{array}{l}\text { Codierter } \\
\text { Mittelwert }\end{array}$} & \multicolumn{4}{|c|}{ Häufigkeiten in \% } & \multirow[b]{2}{*}{$\begin{array}{l}\text { Codierter } \\
\text { Mittelwert }\end{array}$} \\
\hline & Immer & Meistens & $\begin{array}{c}\text { Manch } \\
\text { mal }\end{array}$ & $\mathrm{Nie}$ & & Immer & Meistens & $\begin{array}{c}\text { Manch } \\
\text { mal }\end{array}$ & $\mathrm{Nie}$ & \\
\hline & 38.9 & 13.0 & 40.7 & 7.4 & 2.16 & 45.5 & - & 36.4 & 18.2 & 2.27 \\
\hline
\end{tabular}

Tabelle 56 : Angebot von Hilfe.

Die Antwortmöglichkeiten zur Hilfe durch die Sportlehrkräfte wurden mit 1 bis 4 codiert (immer=1, ..., nie=4). Mit Durchschnittswerten von etwa 2 bieten die Sport- 
lehrkräfte im Schnitt also manchmal Hilfe an, obwohl die Verteilungshöhepunkte sich immer unterscheiden. Es sind kaum systematische Unterschiede zu erkennen, jedoch bieten die Männer tendenziell mehr Hilfe an. Während ein kleiner Prozentsatz sogar nie Hilfe gibt, bietet durchschnittlich ein Drittel immer Hilfe an. Insgesamt etwa die Hälfte antwortete jedoch zu den konkreten Maßnahmen, dass sie immer Hilfe anbieten würden und dass individuelle Unterschiede zwischen den Schülern berücksichtigt würden. Vor dem Hintergrund der großen Klassen kann dies bedeuten, dass die Lehrer nicht bei jedem Schüler Hilfestellungen geben können. Umgekehrt muss aber davon ausgegangen werden, dass ein noch zu großer Anteil der Sportlehrkräfte kaum oder keine Hilfe anbietet, und dies u.U. gerade bei leistungsschwachen Schülern der Fall ist. Sie und weniger leistungsstarke Schüler werden es schwerer haben, Freude am Sporttreiben zu finden.

\begin{tabular}{|l|l|c|c|c|c|}
\hline \multirow{4}{*}{ Item } & \multirow{2}{*}{$\begin{array}{c}\text { Antwort- } \\
\text { möglichkeiten }\end{array}$} & \multicolumn{2}{|c|}{ Mittelschule } & \multicolumn{2}{|c|}{ Oberschule } \\
\cline { 3 - 6 } & & Sportlehrer & $\begin{array}{c}\text { Sportlehre- } \\
\text { rinnen }\end{array}$ & Sportlehrer & $\begin{array}{c}\text { Sportlehre- } \\
\text { rinnen }\end{array}$ \\
\cline { 3 - 6 } & & \multicolumn{2}{|c|}{ Häufigkeiten in \% } & \multicolumn{2}{|c|}{ Häufigkeiten in \% } \\
\hline \hline \multirow{3}{*}{$\begin{array}{l}\text { 70- Bei wel- } \\
\text { chen }\end{array}$} & Gymnastik & 15.2 & 18.8 & 17.7 & 19.1 \\
\cline { 2 - 6 } $\begin{array}{l}\text { Sportarten } \\
\text { bieten Sie } \\
\text { Hilfe an? }\end{array}$ & Fußball & 46.2 & - & 50.0 & - \\
\cline { 2 - 6 } & Handball & 22.1 & 52.9 & 42.6 & 12.6 \\
\cline { 2 - 6 } & Basketball & 18.3 & 64.7 & 37.0 & 54.5 \\
\cline { 2 - 6 } & Volleyball & 27.9 & 64.7 & 46.3 & 45.5 \\
\cline { 2 - 6 } & Leichtathletik & 27.9 & 41.2 & 42.6 & 63.6 \\
\cline { 2 - 6 } & Turnen & 12.4 & 11.1 & 40.7 & 10.7 \\
\hline
\end{tabular}

Tabelle 57: Angebot von Hilfe (nach Sportarten).

In Bezug auf die einzelnen unterrichteten Sportarten ist anhand von Tabelle 57 ersichtlich, dass die Sportlehrerinnen insgesamt etwas häufiger Hilfe anbieten. Dies trifft auch auf das gering ausgeprägte Helfen bei der Gymnastik zu. Sportlehrerinnen ist dabei insbesondere die Vermittlung von Basketball, Volleyball und Leichtathletik ein Anliegen. Bei Männern steht wieder Fußball im Vordergrund, obwohl die Oberschullehrer mit einer Konzentration um 40\% auch in den anderen Sportarten Hilfestellungen leisten (auch im Turnen!). In vielen Sportarten bieten aber weniger als die Hälfte der Sportlehrkräfte einfache oder komplexe Hilfen bei ihrem Unterrichten an, sodass hier ein Manko festgestellt werden muss, das möglicherweise über die Verknüpfung der Themen Begeisterung und Methodik in der Sportlehrerausbildung verringert werden kann. 


\begin{tabular}{|c|c|c|c|c|c|c|c|c|c|c|}
\hline Item & & & & & & & & & & \\
\hline \multirow{9}{*}{$\begin{array}{l}71 \text { - Sind } \\
\text { Sie ein } \\
\text { gutes Vor- } \\
\text { bild, indem } \\
\text { Sie Ihre } \\
\text { Begeiste- } \\
\text { rung für } \\
\text { den Sport } \\
\text { zum Aus- } \\
\text { druck brin- } \\
\text { gen? }\end{array}$} & \multicolumn{10}{|c|}{ Mittelschule } \\
\hline & \multicolumn{5}{|c|}{ Sportlehrer } & \multicolumn{5}{|c|}{ Sportlehrerinnen } \\
\hline & \multicolumn{4}{|c|}{ Häufigkeiten in \% } & \multirow[b]{2}{*}{$\begin{array}{l}\text { Codierter } \\
\text { Mittelwert }\end{array}$} & \multicolumn{4}{|c|}{ Häufigkeiten in \% } & \multirow[b]{2}{*}{$\begin{array}{l}\text { Codierter } \\
\text { Mittelwert }\end{array}$} \\
\hline & $\begin{array}{l}\text { Ja, } \\
\text { immer }\end{array}$ & Meistens & $\begin{array}{c}\text { Manch } \\
\text { mal }\end{array}$ & $\mathrm{Nie}$ & & $\begin{array}{l}\text { Ja, } \\
\text { im- } \\
\text { mer }\end{array}$ & Meistens & $\begin{array}{c}\text { Manch } \\
\text { mal }\end{array}$ & $\mathrm{Nie}$ & \\
\hline & 45.2 & $\overline{12.5}$ & 40.4 & 1.9 & $\overline{1.99}$ & 82.4 & 5.9 & 111.8 & - & $\overline{1.29}$ \\
\hline & \multicolumn{10}{|c|}{ Oberschule } \\
\hline & \multicolumn{5}{|c|}{ Sportlehrer } & \multicolumn{5}{|c|}{ Sportlehrerinnen } \\
\hline & \multicolumn{4}{|c|}{ Häufigkeiten in \% } & & \multicolumn{4}{|c|}{ Häufigkeiten in \% } & \multirow[b]{2}{*}{$\begin{array}{l}\text { Codierter } \\
\text { Mittelwert }\end{array}$} \\
\hline & $\begin{array}{l}\text { Ja, } \\
\text { immer }\end{array}$ & Meistens & $\begin{array}{c}\text { Manch } \\
\text { mal }\end{array}$ & $\mathrm{Nie}$ & $\begin{array}{l}\text { Codierter } \\
\text { Mittelwert }\end{array}$ & $\begin{array}{l}\text { Ja, } \\
\text { im- } \\
\text { mer }\end{array}$ & Meistens & $\begin{array}{c}\text { Manch } \\
\text { mal }\end{array}$ & $\mathrm{Nie}$ & \\
\hline & 46.3 & 9.3 & 33.3 & 11.1 & 2.09 & 27.3 & 18.2 & $\overline{36.4}$ & 18.2 & 2.45 \\
\hline
\end{tabular}

Tabelle 58: Sportlehrerinnen und Sportlehrer als „Vorbild durch Begeisterung“.

Wie bei Item 69 wurde zum Ausdrücken der Begeisterung für den Sport von 1-4 codiert und der Mittelwert liegt wieder insgesamt um „meistens“. Ohne zunächst große erkennbare Unterschiede (Geschlecht und Schulform) sind bis auf die annähernd gleichverteilten Antworten der weiblichen Oberschullehrer die meisten Sportlehrkräfte der Überzeugung, immer ein gutes Vorbild zu sein und der Begeisterung für den Sport Ausdruck zu verleihen. Bei den Sportlehrern fällt auf, dass sie entweder immer oder aber nur manchmal ein Vorbild sind. Unter Berücksichtigung der Verteilung bei den Sportlehrerinnen können also vielleicht verschiedene Lehrertypen gebildet werden. Zu vermuten ist aber, dass das universitäre Ausbildungsziel „Förderung der Persönlichkeit“ bisher noch nicht im Hinblick auf Ausdruck und Charakter in erwünschtem Maße in der Praxis der SportlehrInnen Ägyptens angekommen ist, denn als Maßnahmen nannten je die Hälfte der Sportlehrkräfte Erklärungen und Vormachen der Übungen sowie das Vormachen-Lassen durch einen Schüler. 


\begin{tabular}{|c|c|c|c|c|c|}
\hline \multirow{3}{*}{ Item } & \multirow{3}{*}{$\begin{array}{c}\text { Antwort- } \\
\text { möglichkeiten }\end{array}$} & \multicolumn{2}{|c|}{ Mittelschule } & \multicolumn{2}{|c|}{ Oberschule } \\
\hline & & Sportlehrer & $\begin{array}{l}\text { Sportlehre- } \\
\text { rinnen }\end{array}$ & Sportlehrer & $\begin{array}{c}\text { Sportlehre- } \\
\text { rinnen }\end{array}$ \\
\hline & & \multicolumn{2}{|c|}{ Häufigkeiten in \% } & \multicolumn{2}{|c|}{ Häufigkeiten in \% } \\
\hline \multirow{7}{*}{$\begin{array}{l}72 \text { - Bei welchen } \\
\text { Sportarten sind } \\
\text { Sie ein gutes } \\
\text { Vorbild und be- } \\
\text { geistern die Schü- } \\
\text { ler dadurch? }\end{array}$} & Gymnastik & 12.7 & $\overline{10.1}$ & 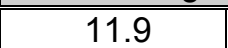 & 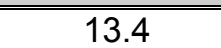 \\
\hline & Fußball & 51.9 & - & 51.9 & - \\
\hline & Handball & 22.1 & 64.7 & 42.6 & 9.6 \\
\hline & Basketball & 27.9 & 47.1 & 38.9 & 27.3 \\
\hline & Volleyball & 24.0 & 70.6 & 29.6 & 54.5 \\
\hline & Leichtathletik & 33.7 & 47.1 & 38.9 & 63.6 \\
\hline & Turnen & 12.5 & 29.4 & 15.7 & 11.9 \\
\hline
\end{tabular}

Tabelle 59 : Vorbild-Sein (nach Sportarten).

Die Verteilung der Antworten zum Vorbild-Sein, aufgeschlüsselt nach Sportarten, ähneln stark der aus Item 70 (Hilfe nach Sportarten). Stichpunktartig: Frauen insgesamt gering häufiger Vorbild als Männer, besonders bei Basketball, Volleyball und Leichtathletik, Männer präferieren Fußball, sind in Oberschule um 40\% verteilt. Da auch hier meist weniger als die Hälfte in den Sportarten ein Vorbild zu sein scheint, können zwei Vermutungen angestellte werden. Erstens, Vorbild-Sein hat für die Sportlehrer etwas mit Vormachen und Helfen zu tun. Zweitens, die Schüler kommen durch zu viele mangelnde Vorbilder auch in der Mehrzahl der Sportarten zu nur wenig Begeisterung (und für den Sport insgesamt). Auch hier spiegelt sich das Bild der in Ägypten traditionellen, geförderten und in der öffentlichen Wahrnehmung wichtigen Sportarten wider: Fußball, Leichtathletik, Basketball und Volleyball (siehe auch Kapitel 2.2) sind für die Lehrer nach Können und Wichtigkeit die zentralen Sportarten. Insgesamt verweist dies wiederum auf eine mögliche Adaption der Ausbildung.

\begin{tabular}{|c|c|c|c|c|}
\hline Item & & & & \\
\hline \multirow{10}{*}{73 - Belohnen Sie besonders gute Leistungen? } & \multicolumn{4}{|c|}{ Mittelschule } \\
\hline & \multirow{2}{*}{\multicolumn{2}{|c|}{$\begin{array}{c}\text { Sportlehrer } \\
\text { Häufigkeiten in \% } \\
\end{array}$}} & \multirow{2}{*}{\multicolumn{2}{|c|}{\begin{tabular}{|c|} 
Sportlehrerinnen \\
Häufigkeiten in \%
\end{tabular}}} \\
\hline & & & & \\
\hline & $\mathrm{Ja}$ & Nein & $\mathrm{Ja}$ & Nein \\
\hline & 85.6 & 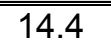 & 94.1 & $\overline{5.9}$ \\
\hline & \multicolumn{4}{|c|}{ Oberschule } \\
\hline & \multirow{2}{*}{\multicolumn{2}{|c|}{$\begin{array}{c}\text { Sportlehrer } \\
\text { Häufigkeiten in \% }\end{array}$}} & \multirow{2}{*}{\multicolumn{2}{|c|}{$\begin{array}{c}\text { Sportlehrerinnen } \\
\text { Häufiakeiten in \% }\end{array}$}} \\
\hline & & & & \\
\hline & $\mathrm{Ja}$ & Nein & $\mathrm{Ja}$ & Nein \\
\hline & 75.9 & 24.1 & 54.5 & 45.5 \\
\hline
\end{tabular}

Tabelle 60: Belohnen besonders guter Leistungen. 
Besonders in der Mittelschule belohen die Lehrer ihre Schüler für besonders gute Leistungen. Die Ergebnisse zur Unterstützung der Akzeptanz erfolgreicher Schüler zeigen sich auch hier, denn das Item erhält ebenfalls eine sehr hohe Zustimmung. Besonders gute Schüler finden Annerkennung durch Lehrer und Klassenkameraden. Um dieser Anerkennung Ausdruck zu verleihen, äußert die Hälfte der Sportlehrkräfte nur eine Anerkennung, wenn sie auch wirklich der Meinung sind, dass die Leistung auch gut war, gleiche $50 \%$ belohnen Leistungen bei einer Feier und ein Drittel versucht diese Schüler an Vereine zu vermitteln.

\begin{tabular}{|c|c|c|c|c|c|c|c|c|c|c|}
\hline Item & & & & & & & & & & \\
\hline \multirow{10}{*}{$\begin{array}{l}74 \text { - Berück- } \\
\text { sichtigen Sie } \\
\text { die Bedürfnis- } \\
\text { se der Schü- } \\
\text { ler? }\end{array}$} & \multicolumn{10}{|c|}{ Mittelschule } \\
\hline & \multicolumn{5}{|c|}{ Sportlehrer } & \multicolumn{5}{|c|}{ Sportlehrerinnen } \\
\hline & \multicolumn{4}{|c|}{ Häufigkeiten in \% } & \multirow[b]{2}{*}{$\begin{array}{l}\text { Codierter } \\
\text { Mittelwert }\end{array}$} & \multicolumn{4}{|c|}{ Häufigkeiten in \% } & \multirow[b]{2}{*}{$\begin{array}{l}\text { Codierter } \\
\text { Mittelwer }\end{array}$} \\
\hline & Immer & Meistens & $\begin{array}{c}\text { Manch } \\
\text { mal }\end{array}$ & Nie & & Immer & Meistens & $\begin{array}{c}\text { Manch } \\
\text { mal }\end{array}$ & $\mathrm{Nie}$ & \\
\hline & 10.6 & 21.2 & 34.6 & 33.7 & 2.91 & - & 11.8 & 88.2 & - & 2.88 \\
\hline & \multicolumn{10}{|c|}{ Oberschule } \\
\hline & \multicolumn{5}{|c|}{ Sportlehrer } & \multicolumn{5}{|c|}{ Sportlehrerinnen } \\
\hline & \multicolumn{4}{|c|}{ Häufigkeiten in \% } & & \multicolumn{4}{|c|}{ Häufigkeiten in \% } & \multirow{2}{*}{$\begin{array}{l}\text { Codierter } \\
\text { Mittelwert }\end{array}$} \\
\hline & Immer & Meistens & $\begin{array}{c}\text { Manch } \\
\text { mal }\end{array}$ & $\mathrm{Nie}$ & Mittelwert & Immer & Meistens & $\begin{array}{c}\text { Manch } \\
\text { mal }\end{array}$ & $\mathrm{Nie}$ & \\
\hline & 25.9 & 11.6 & 48.1 & 14.8 & 2.52 & 45.5 & - & 54.5 & - & 2.19 \\
\hline
\end{tabular}

Tabelle 61: Berücksichtigung von Bedürfnissen der Schüler.

Insgesamt berücksichtigen die Lehrer nach eigener Aussage zu durchschnittlich „meistens“ bis „manchmal“ die Bedürfnisse der Schüler. Da „immer“ oder „meistens“ nicht sehr häufig genannt wurden, kann hier ein Mangel festgestellt werden, wenngleich die Oberschullehrerinnen zu fast der Hälfte immer die Bedürfnisse ihrer Schülerinnen berücksichtigen. Scheinbar geht es den Sportlehrkräften insgesamt um eine strenge Durchführung des Unterrichts, um unkontrolliertem und emotionalem Toben Einhalt zu gebieten. Etwa die Hälfte derjenigen, die die Bedürfnisse berücksichtigen, fragen die Schüler, welche Sportarten sie mögen. Ein gutes Drittel ergreift als Maßnahme Wettkämpfe in verschiedenen Sportarten.

Die Vermittlung des Erziehungszieles Freude/Spaß/Begeisterung wurde mit bestimmten Lehrerhandlungen in Verbindung gebracht. Zusammenfassend seien folgende Punkte hervorgehoben: Bei nur geringen Unterschieden zwischen den Geschlechtern und Schulformen bieten die Sportlehrerinnen etwas mehr Hilfe bei 
einer breiteren Palette an Sportarten an. Das gleiche gilt für das Vorbild-Sein, das inhaltlich mit den Hilfestellungen von den Sportlehrkräften in Verbindung gebracht wird. Während sich dies stärker in den traditionellen und „förderungswürdigen“ Sportarten zeigen, ist die Vermittlung von Freude / Spaß / Begeisterung in diesen beiden Aspekte aber noch relativ gering ausgeprägt (durchschnittlich unter 50\%). Da die Bedürfnisse der Schüler weiterhin wenig berücksichtigt werden, ist auf eine rigide Unterrichtsstrukturierung zu schließen, wobei die wichtigsten Fördermaßnahmen der einzelnen Aspekte in einzelnen Gesprächen und wenig praktischen Maßnahmen liegen. Demgegenüber werden positive Rückmeldungen häufig gegeben und gute Leistungen durch Anerkennungen belohnt. Mit den Ergebnissen ist zu folgern, dass ägyptische Schüler im Sportunterricht am ehesten an Fußball, Basketball, Volleyball und Leichtathletik Freude entwickeln; leistungsstarke Schüler können in jeder Sportart durch Anerkennung „Spaß am Sport“ haben.

\subsubsection{Methodisches Vorgehen}

Vor dem Hintergrund vielfach schon angerissener Maßnahmen seien nun die Ergebnissen zum methodischen Vorgehen im Einzelnen wiedergegeben. Sie können die Erkenntnisse aus dem vorangegangenen Kapitel vertiefen, ergänzen, modifizieren oder relativieren. Hauptaugenmerk liegt aber auf den strukturellen Aspekten.

\section{Allgemeine Methodik}

\begin{tabular}{|c|c|c|c|c|c|c|c|c|c|c|}
\hline Item & & & & & & & & & & \\
\hline \multirow{10}{*}{$\begin{array}{l}75 \text { - Machen } \\
\text { Sie in der } \\
\text { Praxis die } \\
\text { verschiedenen } \\
\text { Übungen vor? }\end{array}$} & \multicolumn{10}{|c|}{ Mittelschule } \\
\hline & \multicolumn{5}{|c|}{ Sportlehrer } & \multicolumn{5}{|c|}{ Sportlehrerinnen } \\
\hline & \multicolumn{4}{|c|}{ Häufigkeiten in \% } & \multirow{2}{*}{$\begin{array}{l}\text { Codierter } \\
\text { Mittelwert }\end{array}$} & \multicolumn{4}{|c|}{ Häufigkeiten in \% } & \multirow{2}{*}{$\begin{array}{l}\text { Codierter } \\
\text { Mittelwert }\end{array}$} \\
\hline & Immer & Meistens & $\begin{array}{c}\text { Manch } \\
\text { mal }\end{array}$ & $\mathrm{Nie}$ & & Immer & Meistens & $\begin{array}{c}\text { Manch } \\
\text { mal }\end{array}$ & $\mathrm{Nie}$ & \\
\hline & 14.4 & 16.3 & 35.6 & 33.7 & 2.88 & 64.7 & 5.9 & 29.4 & - & 1.65 \\
\hline & \multicolumn{10}{|c|}{ Oberschule } \\
\hline & \multicolumn{5}{|c|}{ Sportlehrer } & \multicolumn{5}{|c|}{ Sportlehrerinnen } \\
\hline & \multicolumn{4}{|c|}{ Häufigkeiten in \% } & \multirow[b]{2}{*}{$\begin{array}{l}\text { Codierter } \\
\text { Mittelwert }\end{array}$} & \multicolumn{4}{|c|}{ Häufigkeiten in \% } & \multirow[b]{2}{*}{$\begin{array}{l}\text { Codierter } \\
\text { Mitte/wert }\end{array}$} \\
\hline & Immer & Meistens & $\begin{array}{c}\text { Manch } \\
\text { mal }\end{array}$ & $\mathrm{Nie}$ & & Immer & Meistens & $\begin{array}{c}\text { Manch } \\
\text { mal }\end{array}$ & $\mathrm{Nie}$ & \\
\hline & 29.6 & 13.0 & 33.3 & $\overline{24.1}$ & 2.52 & 36.4 & $\overline{-1}$ & 445.5 & $\overline{18.2}$ & 2.45 \\
\hline
\end{tabular}

Tabelle 62: Vormachen von Übungen. 
Bei nicht erkennbaren Unterschieden zwischen den Schulformen lässt sich das Vormachen der Übungen durch die Sportlehrkräfte wie folgt charakterisieren: Sie geben hauptsächlich ,immer' oder ,manchmal' an, wobei die jungen Sportlehrerinnen der Mittelschule hervorstechen. Von den übrigen machen gar 20-30\% die Übungen nie vor. Entweder machen hier (gute) Schüler die Übungen vor oder die Anweisungen erfolgen nur mündlich. Das Lernen durch Beobachten (z.B. der Akzentuierung bestimmter Bewegungsteile) wird wahrscheinlich in zu vielen Fällen durch die Art der Methodik verhindert.

\begin{tabular}{|c|c|c|c|c|c|}
\hline \multirow{3}{*}{ Item } & \multirow{3}{*}{$\begin{array}{c}\text { Antwort- } \\
\text { möglichkeiten }\end{array}$} & \multicolumn{2}{|c|}{ Mittelschule } & \multicolumn{2}{|c|}{ Oberschule } \\
\hline & & Sportlehrer & $\begin{array}{l}\text { Sportlehre- } \\
\text { rinnen }\end{array}$ & Sportlehrer & $\begin{array}{l}\text { Sportlehre- } \\
\text { rinnen }\end{array}$ \\
\hline & & \multicolumn{2}{|c|}{ Häufigkeiten in \% } & \multicolumn{2}{|c|}{ Häufigkeiten in \% } \\
\hline \multirow{7}{*}{$\begin{array}{l}76 \text { - Bei welcher } \\
\text { Sportart ma- } \\
\text { chen Sie Übun- } \\
\text { gen vor? }\end{array}$} & Gymnastik & 73.6 & 68.3 & 81.3 & 72.8 \\
\hline & Fußball & 67.3 & - & 53.7 & - \\
\hline & Handball & 37.5 & 76.5 & 37.0 & 63.6 \\
\hline & Basketball & 19.2 & 70.6 & 24.1 & 36.4 \\
\hline & Volleyball & 23.1 & 70.6 & 31.5 & 36.4 \\
\hline & Leichtathletik & 34.6 & 70.6 & 29.6 & 54.5 \\
\hline & Turnen & 17.8 & 23.5 & 18.5 & 12.7 \\
\hline
\end{tabular}

Tabelle 63: Vormachen von Übungen (nach Sportarten).

Ähnlich der Spezifizierung von Aspekten der Erziehungsziele auf Sportarten, tauchen auch beim Vormachen praktisch keine systematischen Unterschiede zwischen den Schulformen auf und die Sportarten sind vergleichbar akzentuiert (Männer eher Fußball, Frauen Basketball, Volleyball und Leichtathletik, zusätzlich allerdings noch Handball). Die Sportlehrerinnen machen häufiger in den einzelnen Sportarten vor, wobei insgesamt das überaus häufige Vormachen bei der Gymnastik auffällt (dies zeigte sich auch bei der Kontrollfrage aus Item 75, wobei ca. $30 \%$ angaben, dass sie Gymnastik vormachen, wie es im Lehrplan steht). Während bei den Sportlehrern mit Fußball und Gymnastik nur zwei Sportarten über $50 \%$ kommen, machen über die Hälfte der Sportlehrerinnen bei drei bis fünf Sportarten vor. Zusammen mit obigen Ergebnissen ist zu vermuten, dass bei der Gymnastik viele Sportlehrkräfte immer vormachen und in den anderen Sportarten deutliche Defizite zu erkennen sind, die bei den Frauen etwas geringer ausfallen. 


\begin{tabular}{|c|c|c|c|c|c|c|c|c|c|c|}
\hline Item & & & & & & & & & & \\
\hline \multirow{10}{*}{$\begin{array}{l}77 \text { - Geben Sie } \\
\text { Rückmeldun- } \\
\text { gen (richtig - } \\
\text { falsch) während } \\
\text { der } \\
\text { Übungen? }\end{array}$} & \multicolumn{10}{|c|}{ Mittelschule } \\
\hline & \multicolumn{5}{|c|}{ Sportlehrer } & \multicolumn{5}{|c|}{ Sportlehrerinnen } \\
\hline & \multicolumn{4}{|c|}{ Häufigkeiten in \% } & \multirow{2}{*}{$\begin{array}{l}\text { Codierter } \\
\text { Mittelwert }\end{array}$} & \multicolumn{4}{|c|}{ Häufigkeiten in \% } & \multirow[b]{2}{*}{$\begin{array}{l}\text { Codierter } \\
\text { Mittelwert }\end{array}$} \\
\hline & Immer & Meistens & $\begin{array}{c}\text { Manch } \\
\text { mal }\end{array}$ & $\mathrm{Nie}$ & & Immer & Meistens & $\begin{array}{c}\text { Manch } \\
\text { mal }\end{array}$ & $\mathrm{Nie}$ & \\
\hline & 30.8 & 17.3 & 33.7 & 18.3 & 2.39 & 47.1 & 5.9 & 47.1 & - & 2.00 \\
\hline & \multicolumn{10}{|c|}{ Oberschule } \\
\hline & \multicolumn{5}{|c|}{ Sportlehrer } & \multicolumn{5}{|c|}{ Sportlehrerinnen } \\
\hline & \multicolumn{4}{|c|}{ Häufigkeiten in \% } & \multirow{2}{*}{$\begin{array}{l}\text { Codierter } \\
\text { Mittelwert }\end{array}$} & \multicolumn{4}{|c|}{ Häufigkeiten in \% } & \multirow{2}{*}{$\begin{array}{l}\text { Codierter } \\
\text { Mittelwert }\end{array}$} \\
\hline & Immer & Meistens & $\begin{array}{c}\text { Manch } \\
\text { mal }\end{array}$ & Nie & & Immer & Meistens & $\begin{array}{c}\text { Manch } \\
\text { mal }\end{array}$ & $\mathrm{Nie}$ & \\
\hline & 40.7 & 13.0 & 35.2 & $\overline{11.1}$ & 2.17 & 54.5 & 9.1 & 36.4 & $\overline{-1}$ & 1.81 \\
\hline
\end{tabular}

Tabelle 64: Rückmeldung durch die Sportlehrkräfte in ihrem Unterricht.

Durchaus vergleichbar mit den Ergebnissen zum Vormachen, können auch bei den Rückmeldungen zwei Konzentrationen festgestellt, nämlich auf ,immer' und ,manchmal'. Diesmal geben die Sportlehrerinnen aber durchgehend häufiger ,immer' an, sodass hier positivere Mittelwerte resultieren. Obwohl diese Daten insgesamt zuversichtlich stimmen (etwa die Hälfte der Sportlehrkräfte geben häufiger Rückmeldungen), sei darauf aufmerksam gemacht, dass ein Drittel bis die Hälfte der Sportlehrkräfte nur manchmal Rückmeldungen geben.

Etwa die Hälfte derjenigen, die Rückmeldungen geben, nennen als Maßnahmen Beobachtungen und Korrekturen von Fehlern bei der Gymnastik und gut zwei Drittel wollen gar alle Fehler bei den Übungen in ihrem Sportunterricht korrigieren.

\begin{tabular}{|l|l|c|c|c|c|}
\hline \multirow{4}{*}{ Item } & \multicolumn{1}{|c|}{$\begin{array}{c}\text { Antwort- } \\
\text { möglichkeiten }\end{array}$} & \multicolumn{2}{|c|}{ Mittelschule } & \multicolumn{2}{|c|}{ Oberschule } \\
\cline { 3 - 6 } & & Sportlehrer & $\begin{array}{c}\text { Sportlehre- } \\
\text { rinnen }\end{array}$ & Sportlehrer & $\begin{array}{c}\text { Sportlehre- } \\
\text { rinnen }\end{array}$ \\
\cline { 3 - 6 } & Gäufigkeiten in \% & Häufigkeiten in \% \\
\hline \hline \multirow{4}{*}{$\begin{array}{l}\text { 78- Bei welcher } \\
\text { Sportart geben Sie } \\
\text { Rückmeldungen? }\end{array}$} & Gymnastik & 55.4 & 69.1 & 57.9 & 63.3 \\
\cline { 2 - 6 } & Fußball & 56.7 & - & 59.3 & - \\
\cline { 2 - 6 } & Handball & 40.4 & 70.6 & 40.7 & 100.0 \\
\cline { 2 - 6 } & Basketball & 35.6 & 69.6 & 46.3 & 27.3 \\
\cline { 2 - 6 } & Lolleyball & 28.9 & 58.8 & 44.4 & 45.5 \\
\cline { 2 - 6 } & Leichtathletik & 46.2 & 41.2 & 58.3 & 54.5 \\
\cline { 2 - 6 } & Turnen & 26.0 & 47.1 & 37.0 & 18.2 \\
\hline
\end{tabular}

Tabelle 65: Rückmeldung durch die Sportlehrkräfte (nach Sportarten).

Rückmeldungen in den einzelnen Sportarten geben vergleichsweise viele Sportlehrkräfte. Das Verhältnis von über $50 \%$ : unter $50 \%$ liegt bei $12: 14$ und die Geschlechter ähneln sich hier stark. Es zeigt sich eine Tendenz wie beim Vorma- 
chen, jedoch tritt bei den Sportlehrerinnen Leichtathletik hinzu. Die Gymnastik hat auch bei den Rückmeldungen einen hohen Stellenwert (siehe auch Item 77) und insgesamt zeigt sich hier das stärkste Votum für einzelne Sportarten. Rückmeldungen werden also zu einem großen Teil im Sportunterricht gegeben, jedoch zeigen die Werte, dass weniger als die Hälfte nur selten Rückmeldungen geben für eine zentrale Methode des Sportunterrichts bedeutend zu wenig.

\begin{tabular}{|c|c|c|c|c|c|c|c|c|c|c|}
\hline Item & & & & & & & & & & \\
\hline \multirow{10}{*}{$\begin{array}{l}79 \text { - Bereiten } \\
\text { Sie die Schü- } \\
\text { ler auf die } \\
\text { Prüfungen } \\
\text { vor? }\end{array}$} & \multicolumn{10}{|c|}{ Mittelschule } \\
\hline & \multicolumn{5}{|c|}{ Sportlehrer } & \multicolumn{5}{|c|}{ Sportlehrerinnen } \\
\hline & \multicolumn{4}{|c|}{ Häufigkeiten in \% } & \multirow[b]{2}{*}{$\begin{array}{l}\text { Codierter } \\
\text { Mittelwert }\end{array}$} & \multicolumn{4}{|c|}{ Häufigkeiten in \% } & \multirow{2}{*}{$\begin{array}{l}\text { Codierter } \\
\text { Mittelwert }\end{array}$} \\
\hline & Immer & Meistens & $\begin{array}{c}\begin{array}{c}\text { Manch } \\
\text { mal }\end{array} \\
\end{array}$ & Nie & & Immer & Meistens & $\begin{array}{c}\text { Manch } \\
\text { mal }\end{array}$ & $\mathrm{Nie}$ & \\
\hline & 13.5 & 1.0 & 34.6 & 51.0 & 3.23 & 52.9 & 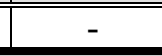 & 35.3 & 11.8 & 2.06 \\
\hline & \multicolumn{10}{|c|}{ Oberschule } \\
\hline & \multicolumn{5}{|c|}{ Sportlehrer } & \multicolumn{5}{|c|}{ Sportlehrerinnen } \\
\hline & \multicolumn{4}{|c|}{ Häufigkeiten in \% } & \multirow[b]{2}{*}{$\begin{array}{l}\text { Codierter } \\
\text { Mittelwert }\end{array}$} & \multicolumn{4}{|c|}{ Häufigkeiten in \% } & \multirow[b]{2}{*}{$\begin{array}{l}\text { Codierter } \\
\text { Mittelwert }\end{array}$} \\
\hline & Immer & Meistens & $\begin{array}{c}\text { Manch } \\
\text { mal }\end{array}$ & Nie & & Immer & Meistens & $\begin{array}{c}\text { Manch } \\
\text { mal }\end{array}$ & $\mathrm{Nie}$ & \\
\hline & 9.3 & 3.7 & 35.2 & 51.9 & 3.29 & 9.1 & - & 72.7 & 18.2 & 3.00 \\
\hline
\end{tabular}

Tabelle 66: Prüfungsvorbereitungen seitens der Sportlehrkräfte.

Die Daten aus Tabelle 66 zeigen, dass die männlichen Sportlehrkräfte eher keine Prüfungsvorbereitungen durchführen. Insgesamt über $80 \%$ der Sportlehrerinnen und Sportlehrer bereiten nur manchmal oder nie auf Prüfungen vor, wobei die jungen Sportlehrerinnen der Mittelschule eine Ausnahme bilden (zur Hälfte immer). Geringfügige Unterschiede lassen sich anhand der Schulformen erkennen, denn die Oberschullehrerlnnen bereiten etwas weniger vor.

Als häufigste Maßnahmen nannten die Lehrkräfte, dass sie Tests nur mit den Schulmannschaften durchführen (etwa 70\%), um 40\% erklären den Schülern, wie wichtig Tests für die Leistungsentwicklung sind (und wie sie sich selbst darauf vorbereiten können) und ca. ein Drittel bereitet die Schüler durch Gespräche in der vorhergehenden Unterrichtseinheit vor. 


\begin{tabular}{|c|c|c|c|c|c|}
\hline \multirow{3}{*}{ Item } & \multirow{3}{*}{$\begin{array}{c}\text { Antwort- } \\
\text { möglichkeiten }\end{array}$} & \multicolumn{2}{|c|}{ Mittelschule } & \multicolumn{2}{|c|}{ Oberschule } \\
\hline & & Sportlehrer & $\begin{array}{l}\text { Sportlehre- } \\
\text { rinnen }\end{array}$ & Sportlehrer & $\begin{array}{l}\text { Sportlehre- } \\
\text { rinnen }\end{array}$ \\
\hline & & \multicolumn{2}{|c|}{ Häufigkeiten in \% } & \multicolumn{2}{|c|}{ Häufigkeiten in \% } \\
\hline \multirow{6}{*}{$\begin{array}{l}\text { 80- Bei welcher } \\
\text { Sportart bereiten } \\
\text { sie die Schüler } \\
\text { auf die Prüfung } \\
\text { vor? }\end{array}$} & Fußball & 477.1 & - & 488.1 & - \\
\hline & Handball & 24.0 & 70.6 & 31.5 & 81.8 \\
\hline & Basketball & 10.7 & 52.9 & 18.5 & 36.4 \\
\hline & Volleyball & 14.4 & 64.7 & 16.7 & 45.5 \\
\hline & Leichtathletik & 32.7 & 69.6 & 29.6 & 18.2 \\
\hline & Turnen & 14.4 & 17.6 & 14.8 & - \\
\hline
\end{tabular}

Tabelle 67: Prüfungsvorbereitungen (nach Sportarten).

Die wenig ausgeprägten Prüfungsvorbereitungen der Männer zeigt sich auch bei den Sportarten. Mit Ausnahme von Fußball bereiten sie nur in einem geringen Teil der Sportarten vor. Die Frauen zeigen wieder eine breitere Streuung, vornehmlich auf die Spielsportarten. Insgesamt ist die Prüfungsvorbereitung im ägyptischen Sportunterricht also gering ausgeprägt, wahrscheinlich werden Prüfungen eher selten durchgeführt ${ }^{21}$.

\begin{tabular}{|l|l|c|c|c|c|}
\hline \multirow{2}{*}{ Item } & \multirow{2}{*}{$\begin{array}{c}\text { Antwort- } \\
\text { möglichkeiten }\end{array}$} & \multicolumn{2}{|c|}{ Mittelschule } & \multicolumn{2}{c|}{ Oberschule } \\
\cline { 3 - 6 } & & Sportlehrer & $\begin{array}{c}\text { Sportlehre- } \\
\text { rinnen }\end{array}$ & Sportlehrer & $\begin{array}{c}\text { Sportlehre- } \\
\text { rinnen }\end{array}$ \\
\cline { 3 - 6 } & & \multicolumn{2}{|c|}{ Häufigkeiten in \% } & \multicolumn{2}{c|}{ Häufigkeiten in \% } \\
\hline \hline \multirow{2}{*}{$\begin{array}{l}\text { 81- Bei wel- } \\
\text { cher Sportart } \\
\text { führen } \\
\begin{array}{l}\text { Sie Wettkämp- } \\
\text { fe durch? }\end{array}\end{array}$} & Fußball & 99.0 & - & 96.3 & - \\
\cline { 2 - 6 } & Handball & 93.3 & 94.1 & 90.7 & 90.9 \\
\cline { 2 - 6 } & Basketball & 89.4 & 77.4 & 88.9 & 77.9 \\
\cline { 2 - 6 } & Leilleyball & 93.3 & 89.2 & 95.3 & 80.9 \\
\cline { 2 - 6 } & Turnen & 76.9 & 90.1 & 87.0 & 81.8 \\
\hline
\end{tabular}

Tabelle 68: Durchführung von Wettkämpfen (nach Sportarten).

Die Durchführung von Wettkämpfen im ägyptischen Sportunterricht (bzw. im Schulsport) scheint überaus beliebt. Mit Ausnahme des Turnens werden in praktisch allen gelehrten Sportarten fast immer Wettkämpfe durchgeführt.

Zusammenfassend lässt sich im Hinblick auf die Bedeutsamkeit der Aspekte allgemeiner Methodik eine Rangfolge ausmachen: 1. Wettkämpfe, 2. Rückmeldungen, 3. Vormachen und 4. Prüfungsvorbereitungen. Während die Wettkämpfe als

${ }^{21}$ In Gymnastik finden keine Prüfungen statt und es werden auch keine Wettkämpfe durchgeführt (nächster Aspekt). Insofern tauchen sie auch nicht in den Listen auf. 
wichtigstes Mittel des Sportunterrichts heraustreten (in praktisch allen Sportarten außer Turnen), fallen die anderen Aspekte deutlich zurück. Rückmeldungen geben lediglich etwa die Hälfte der Sportlehrkräfte, etwas weniger machen vor und vielfach finden keine Prüfungsvorbereitungen statt. Wahrscheinlich verteilen sich die Maßnamen auf die auch bei der Implementierung der Erziehungsziele schwerpunktmäßig herausgetretenen Sportarten (Männer Fußball, Frauen Spielsportarten und insgesamt tendenziell Leichtathletik), wobei während der Gymnastik vielfach nach Lehrplan vorgemacht und korrigiert wird. Wenngleich die Sportlehrerinnen mehr Rückmeldungen geben und auch etwas häufiger auf Prüfungen vorbereiten, werden konkrete Möglichkeiten einer gezielten Verbesserung von Fähigkeiten und Fertigkeiten zu häufig ausgelassen. Deutlich zu viele Sportlehrkräfte antworten bei zentralen Aspekten der Vermittlung nur mit ,manchmal' oder ,nie'.

\section{Methodik bei der sportartspezifischen Fertigkeitsvermittlung}

\begin{tabular}{|c|c|c|c|c|c|}
\hline \multirow{3}{*}{ Item } & \multirow{3}{*}{$\begin{array}{l}\text { Antwort- } \\
\text { möglichkeiten }\end{array}$} & \multicolumn{2}{|c|}{ Mittelschule } & \multicolumn{2}{|c|}{ Oberschule } \\
\hline & & Sportlehrer & $\begin{array}{l}\text { Sportlehre- } \\
\text { rinnen }\end{array}$ & Sportlehrer & $\begin{array}{c}\text { Sportlehre- } \\
\text { rinnen }\end{array}$ \\
\hline & & \multicolumn{2}{|c|}{ Häufigkeiten in \% } & \multicolumn{2}{|c|}{ Häufigkeiten in \% } \\
\hline \multirow{8}{*}{$\begin{array}{l}82 \text { - Welche } \\
\text { Lehrmethoden } \\
\text { setzen Sie bei } \\
\text { der Vermittlung } \\
\text { der im Lehrplan } \\
\text { vorgesehenen } \\
\text { Sportarten ein? }\end{array}$} & $\begin{array}{l}\text { Nur Ganzheitsme- } \\
\text { thode }\end{array}$ & 75.1 & 62.25 & 82.56 & 90.17 \\
\hline & Nur Teilmethode & 96.9 & 87.31 & 93.14 & 89.47 \\
\hline & $\begin{array}{l}\text { Nur Ganz-Teil- } \\
\text { Methode }\end{array}$ & 99.3 & 89.14 & 92.17 & 84.12 \\
\hline & $\begin{array}{l}\text { Sowohl Ganzheits- } \\
\text { methode als auch } \\
\text { Teilmethode }\end{array}$ & 57.4 & 71.11 & 77.24 & 63.25 \\
\hline & $\begin{array}{l}\text { Sowohl Ganzheits- } \\
\text { methode als auch } \\
\text { Ganz-Teil-Methode }\end{array}$ & 33.6 & 46.27 & 53.18 & 60.17 \\
\hline & $\begin{array}{l}\text { Sowohl Teilmetho- } \\
\text { de als auch Ganz- } \\
\text { Teil-Methode }\end{array}$ & 13.1 & 22.13 & 64.01 & 71.43 \\
\hline & Alle Methoden & 31.7 & 55.19 & 72.53 & 80.20 \\
\hline & Andere Methoden & - & - & - & - \\
\hline
\end{tabular}

Tabelle 69: Verwendete Lehrmethode bei der Fertigkeitsvermittlung.

Gute $90 \%$ der Sportlehrkräfte wenden die drei „großen Methoden“ der sportartspezifischen Fertigkeitsvermittlung an. Dies ist bei Frauen und Männern gleichermaßen der Fall. Allerdings geben die Sportlehrerinnen zusätzlich zu einem hohen Prozentsatz Kombinationen von Methoden an (50-80\%). Wie schon bei den Mei- 
nungen der Sportlehrkräfte vermutet, findet nur ein überwiegender Teil einzelner Methoden Eingang in den ägyptischen Sportunterricht - zu ungunsten eines Methodenmixes -, was aber interessanterweise bei den weiblichen Lehrkräften eher nicht der Fall ist.

\begin{tabular}{|c|c|c|c|c|c|}
\hline \multirow{3}{*}{ Item } & \multirow{3}{*}{$\begin{array}{c}\text { Antwort- } \\
\text { möglichkeiten }\end{array}$} & \multicolumn{2}{|c|}{ Mittelschule } & \multicolumn{2}{|c|}{ Oberschule } \\
\hline & & Sportlehrer & $\begin{array}{l}\text { Sportlehre- } \\
\text { rinnen }\end{array}$ & Sportlehrer & $\begin{array}{l}\text { Sportlehre- } \\
\text { rinnen }\end{array}$ \\
\hline & & \multicolumn{2}{|c|}{ Häufigkeiten in \% } & \multicolumn{2}{|c|}{ Häufigkeiten in \% } \\
\hline \multirow{2}{*}{$\begin{array}{l}83 \text { - Welche } \\
\text { Lehrmethoden } \\
\text { setzen Sie über- } \\
\text { wiegenden ein? }\end{array}$} & Individuelle & 11.5 & - & 9.6 & 18.2 \\
\hline & Kollektive & 89.4 & 100.0 & 94.4 & 81.8 \\
\hline
\end{tabular}

Tabelle 70: Individuelle vs. kollektive Unterrichtsmethoden.

Vor dem Hintergrund der Klassengröße werden erwartungsgemäß kollektive Unterrichtsmethoden eingesetzt. Sportlehrerinnen der Mittelschule setzen sie ausschließlich ein. Berücksichtigt man die Ergebnisse zur Lehrermeinung, so ist festzustellen, dass Wunsch und Realität in Bezug auf individuelle Methoden deutlich auseinander klaffen. Daher müssen die dort getätigten Vermutungen eher in Richtung „unmöglich“ korrigiert werden.

Die Methodik bei der sportartspezifischen ist schnell zusammengefasst. Während einzelne Methoden eine breite Anwendung finden, kombinieren die Sportlehrerinnen vielfach auch. Verständlich vor dem Hintergrund der großen Klassen ist im zweiten Aspekt die Anwendung kollektiver Methoden. Wahrscheinlich ist es unmöglich bei 45 Min. pro Unterrichtseinheit und über 40 Schülern gezielt auf einzelne Schüler einzugehen. 


\section{Inhaltliche Schwerpunkte}

\begin{tabular}{|l|l|c|c|c|c|}
\hline \multirow{2}{*}{ Item } & \multirow{2}{*}{$\begin{array}{c}\text { Antwort- } \\
\text { möglichkeiten }\end{array}$} & \multicolumn{2}{|c|}{ Mittelschule } & \multicolumn{2}{c|}{ Oberschule } \\
\cline { 3 - 6 } & & Sportlehrer & $\begin{array}{c}\text { Sportlehre- } \\
\text { rinnen }\end{array}$ & Sportlehrer $\begin{array}{c}\text { Sportlehre- } \\
\text { rinnen }\end{array}$ \\
\cline { 3 - 6 } & & \multicolumn{2}{|c|}{ Häufigkeiten in \% } & Häufigkeiten in \% \\
\hline \hline $\begin{array}{l}84-\text { Welche } \\
\text { Fähigkeiten } \\
\text { fördern Sie } \\
\text { besonders } \\
\text { ausgeprägt? }\end{array}$ & Ausdauerfähigkeiten & 53.8 & 11.8 & 51.9 & 18.2 \\
\cline { 2 - 6 } & Beweglichkeit & 28.8 & 15.9 & 18.5 & 18.2 \\
\cline { 2 - 6 } & Schnelligkeit & 51.9 & 11.8 & 35.2 & 63.6 \\
\hline
\end{tabular}

Tabelle 71: Inhaltliche Schwerpunkte (Fähigkeiten).

Bei der Möglichkeit zur Mehrfachnennung gaben über die Hälfte der Sportlehrer an, besonders ausgeprägt Ausdauer zu fördern, wobei sich sonst ein eher diffuses Bild zeigt. Mit Blick auf die Schulformen ergaben sich nur punktuelle Unterschiede (hervorzuheben: 70\% der Mittelschullehrerinnen Koordinative Fähigkeiten, 60\% der Oberschullehrerinnen Schnelligkeit).

\begin{tabular}{|c|c|c|c|c|c|}
\hline \multirow{3}{*}{ Item } & \multirow{3}{*}{$\begin{array}{c}\text { Antwort- } \\
\text { möglichkeiten }\end{array}$} & \multicolumn{2}{|c|}{ Mittelschule } & \multicolumn{2}{|c|}{ Oberschule } \\
\hline & & Sportlehrer & $\begin{array}{c}\text { Sportlehre- } \\
\text { rinnen }\end{array}$ & Sportlehrer & $\begin{array}{c}\text { Sportlehre- } \\
\text { rinnen }\end{array}$ \\
\hline & & \multicolumn{2}{|c|}{ Häufigkeiten in \% } & \multicolumn{2}{|c|}{ Häufigkeiten in \% } \\
\hline \multirow{3}{*}{$\begin{array}{l}85 \text { - Gibt es bei } \\
\text { der Vermittlung } \\
\text { von Fertigkeiten } \\
\text { in Ihrem Unter- } \\
\text { richt } \\
\text { Schwerpunkte? }\end{array}$} & $\begin{array}{l}\text { Ich vermittle mehr moto- } \\
\text { rische Grundfertigkeiten } \\
\text { (wie Werfen, Springen, } \\
\text { Drehungen, Treffen). }\end{array}$ & 12.8 & 11.8 & 15.0 & 18.2 \\
\hline & $\begin{array}{l}\text { Ich vermittle mehr sport- } \\
\text { artspezifische Fertigkei- } \\
\text { ten (z.B. Kopfball im } \\
\text { Fußball, Sprungwurf im } \\
\text { Handball, Korbleger im } \\
\text { Basketball, Speerwurf). }\end{array}$ & 10.6 & 11.8 & 9.1 & 27.3 \\
\hline & $\begin{array}{l}\text { Ich vermittle beide Fertig- } \\
\text { keiten zu gleichen Teilen. }\end{array}$ & 81.7 & 76.5 & 75.9 & 54.5 \\
\hline
\end{tabular}

Tabelle 72: Inhaltliche Schwerpunkte (Fertigkeiten).

Etwa 10 bis 20\% der Sportlehrkräfte haben im Hinblick auf Fertigkeitsvermittlung Präferenzen (eher motorische Grundfertigkeiten oder eher sportartspezifische Fertigkeiten). Der überwiegende Teil (etwa drei Viertel) vermittelt jedoch beide Fertigkeiten zu gleichen Teilen. Dies ist bei den Männern ausgeprägter als bei den Frauen. 
Im Untersuchungsaspekt inhaltliche Schwerpunkte kommt bei der weitgehend erkannten breiten Zielsetzung also folgenden zwei Aspekten eine hervorgehobene Bedeutung zu. Erstens werden die Ausdauerfähigkeiten von den männlichen Lehrern bevorzugt, was auf ein spezielles Gesundheitsverständnis verweisen könnte. Zweitens findet meist eine gleichberechtigte Vermittlung von Fertigkeiten statt auch in der Oberschule, wo eigentlich mehr sportspezifische Fertigkeiten geschult werden sollen aber nach Meinung der Lehrer gleichberechtigt angewendet werden wollen (siehe Kapitel 5.1.2, 3.).

\section{Bewertungsmethoden}

\begin{tabular}{|c|c|c|c|c|}
\hline Item & & & & \\
\hline \multirow{10}{*}{$\begin{array}{l}86 \text { - Bewerten sie den Leistungsstand oder die } \\
\text { Leistungsentwicklung der Schüler nach objekti- } \\
\text { ven Kriterien? }\end{array}$} & \multicolumn{4}{|c|}{ Mittelschule } \\
\hline & \multicolumn{2}{|c|}{ Sportlehrer } & \multicolumn{2}{|c|}{ Sportlehrerinnen } \\
\hline & \multicolumn{2}{|c|}{ Häufigkeiten in \% } & \multicolumn{2}{|c|}{ Häufigkeiten in \% } \\
\hline & $\mathrm{Ja}$ & Nein & $\mathrm{Ja}$ & Nein \\
\hline & 46.2 & 53.8 & 48.8 & 51.2 \\
\hline & \multicolumn{4}{|c|}{ Oberschule } \\
\hline & \multicolumn{2}{|c|}{ Sportlehrer } & \multicolumn{2}{|c|}{ Sportlehrerinnen } \\
\hline & \multicolumn{2}{|c|}{ Häufigkeiten in \% } & \multicolumn{2}{|c|}{ Häufigkeiten in \% } \\
\hline & $\mathrm{Ja}$ & Nein & $\mathrm{Ja}$ & Nein \\
\hline & 39.9 & 60.1 & 35.7 & 64.3 \\
\hline
\end{tabular}

Tabelle 73: Bewertungen im Unterricht.

Der überwiegende Teil der Sportlehrkräfte gibt an, keine Bewertungen im Unterricht vorzunehmen, aber je die Hälfte der Mittelschullehrer tut dies nach objektiven Kriterien oder eben nicht. Etwas ausgeprägter ist dies allerdings in den Oberschulen, wo die Bewertungspraxis zu zwei Dritteln nicht erkennbar ist. 


\begin{tabular}{|c|c|c|c|c|c|}
\hline \multirow{3}{*}{ Item } & \multirow{3}{*}{$\begin{array}{l}\text { Antwort- } \\
\text { möglichkeiten }\end{array}$} & \multicolumn{2}{|c|}{ Mittelschule } & \multicolumn{2}{|c|}{ Oberschule } \\
\hline & & Sportlehrer & $\begin{array}{l}\text { Sportlehre- } \\
\text { rinnen }\end{array}$ & Sportlehrer & $\begin{array}{c}\text { Sportlehre- } \\
\text { rinnen }\end{array}$ \\
\hline & & \multicolumn{2}{|c|}{ Häufigkeiten in \% } & \multicolumn{2}{|c|}{ Häufigkeiten in \% } \\
\hline \multirow{3}{*}{$\begin{array}{l}87- \\
\text { Wenn } \\
\text { ja, wie } \\
\text { kommen } \\
\text { Sie zum } \\
\text { Bewer- } \\
\text { tung- } \\
\text { sergeb- } \\
\text { nis? }\end{array}$} & $\begin{array}{l}\text { Durch regelmäßige } \\
\text { Zwischenbewertungen }\end{array}$ & 22.9 & 41.2 & 31.5 & 54.5 \\
\hline & \begin{tabular}{|l|} 
Durch Zwischenbe- \\
wertungen und End- \\
bewertung
\end{tabular} & 29.8 & 47.1 & 29.6 & 18.2 \\
\hline & Durch Endbewertung & 48.1 & 11.8 & 39.0 & 27.3 \\
\hline
\end{tabular}

Tabelle 74: Bewertungsstile.

Von insgesamt der Hälfte bzw. einem Drittel der Sportlehrkräfte wurden dann die Bewertungsstile erfragt. Anhand von Tabelle 74 lässt sich erkennen, dass die Männer stärker durch Endbewertung zu einem Ergebnis kommen und Frauen stärker durch Zwischenbewertungen. Die Tatsache, dass aber nur 40-50\% überhaupt bewerten, mag ihre Ursache im fehlenden Niederschlag des Sportunterrichts als Note im Zeugnis haben. Obwohl an den Universitäten explizit unterrichtet, im Lehrplan aufgegriffen und - wie im folgenden Abschnitt zu sehen sein wird auch die materiellen Bedingungen gegeben, scheinen die Sportlehrkräfte wenig motiviert, überhaupt Bewertung als Baustein des Unterrichts zu verwenden. Vor diesem Hintergrund lässt sich auch die wenig ausgeprägte Prüfungsvorbereitung erklären. Dort wurde auch ersichtlich, dass die prüfenden Sportlehrkräfte zu fast drei Viertel nur im Schulsport Tests durchführen und deren Bedeutung thematisieren wollen.

\subsubsection{Rahmenbedingungen und Hilfsmittel}

Die Rahmenbedingungen und Hilfsmittel wurden getrennt erhoben. Im Einzelnen wird wiedergegeben, welche essenziellen materiellen und organisatorischen Bedingungen gegeben sind und welche Hilfsmittel vorliegen, die jeweils aus Sicht des Lehrplans notwendig wären. 


\begin{tabular}{|c|c|c|c|c|c|}
\hline \multirow{3}{*}{ Item } & \multirow{3}{*}{$\begin{array}{l}\text { Antwort- } \\
\text { möglichkeiten }\end{array}$} & \multicolumn{2}{|c|}{ Mittelschule } & \multicolumn{2}{|c|}{ Oberschule } \\
\hline & & Sportlehrer & $\begin{array}{l}\text { Sportlehre- } \\
\text { rinnen }\end{array}$ & Sportlehrer & $\begin{array}{l}\text { Sportlehre- } \\
\text { rinnen }\end{array}$ \\
\hline & & \multicolumn{2}{|c|}{ Häufigkeiten in \% } & \multicolumn{2}{|c|}{ Häufigkeiten in \% } \\
\hline \multirow{6}{*}{$\begin{array}{l}88 \text { - Wel- } \\
\text { che Rah- } \\
\text { menbedin- } \\
\text { gungen } \\
\text { liegen } \\
\text { tatsächlich } \\
\text { für Ihren } \\
\text { Sportunter- } \\
\text { richt vor? }\end{array}$} & $\begin{array}{l}\text { Regelmäßige War- } \\
\text { tung der Sportstät- } \\
\text { ten und der dazu- } \\
\text { gehörigen Einrich- } \\
\text { tungen wie Ge- } \\
\text { genstände }\end{array}$ & 59.6 & 29.4 & 57.4 & 63.6 \\
\hline & $\begin{array}{l}\text { Adäquater Um- } \\
\text { kleideraum }\end{array}$ & 18.7 & - & 13.7 & 19.7 \\
\hline & $\begin{array}{l}\text { Ausreichende } \\
\text { Anzahl von Toilet- } \\
\text { ten und Waschbe- } \\
\text { cken }\end{array}$ & 20.2 & 41.2 & 14.8 & 18.2 \\
\hline & $\begin{array}{l}\text { Ärztliche Betreu- } \\
\text { ung bei Krank- } \\
\text { heitsfällen / Unfäl- } \\
\text { len der Schüler }\end{array}$ & 44.2 & 11.8 & 25.9 & 19.6 \\
\hline & $\begin{array}{l}\text { Mehrere Mahlzei- } \\
\text { ten pro Woche für } \\
\text { die Schüler }\end{array}$ & 13.8 & 15.9 & 16.7 & 18.2 \\
\hline & $\begin{array}{l}\text { Ein extra Budget } \\
\text { für den Sportunter- } \\
\text { richt }\end{array}$ & 18.3 & 17.6 & 20.4 & 27.3 \\
\hline
\end{tabular}

Tabelle 75: Rahmenbedingungen des ägyptischen Sportunterrichts.

Tabelle 75 offenbart ein problematisches Bild der Rahmenbedingungen. Obwohl die überwiegende Mehrheit (etwa zwei Drittel) der Sportlehrkräfte angibt, dass eine regelmäßige Wartung der Sportstätten incl. Einrichtungen stattfindet (von der Mittelschullehrerinnen sogar nur etwa ein Drittel), ist dies bedenklich. Vor dem Hintergrund des hohen Alters der Sportstätten (siehe Kapitel 2.3), scheint eine intensive Pflege geboten, damit die Sportstätten nicht verfallen. Mit einem Drittel „Nicht-Pfleger“ ist das Risiko für eine weitere Minderung der Sportgelegenheiten stark erhöht ${ }^{22}$, wie die weiteren Daten zeigen. Über adäquate Umkleideräume verfügen nur 15-20\% der Schulen, die untersuchten Mittelschulen für Mädchen scheinen keine zu haben. Obwohl von der Sportpädagogik gefordert und implizit auch im Lehrplan enthalten, kann dies dazu führen, dass häufig überhaupt keine Sportkleidung getragen wird und auch aus kulturellen Gründen (Verhüllung der Frauen i.w.S.) Nachbesserungen vorgenommen werden müssten. Eine ausrei-

\footnotetext{
${ }^{22}$ Ursachen für die noch zu niedrigen Werte können allerdings vielfältig sein. Beispielsweise könnte dies auch das Frageverständnis der Lehrer sein. Wenn unter ,Wartung' die Aufgaben der Bezirksleiter verstanden wurde, dann müsste man diesen eine mangelhafte Arbeit attestieren.
} 
chende Anzahl an Toiletten und Waschbecken haben im Durchschnitt ca. 20\% der Schulen. Vor dem Hintergrund des Erziehungszieles Hygiene fehlen hier ebenso in einer gravierend hohen Anzahl essenzielle Bedingungen. Auch die Notfallversorgung scheint nur zu einem geringen Teil gesichert. Entweder kümmern sich die Sportlehrkräfte nicht darum (Kontakt zu Ärzten) oder vertrauen auf ihre Verletzungsprophylaxe im Unterricht, was aber mit den geringen Werten zu Hilfestellung etc. tatsächlich nicht sehr ausgeprägt sein kann. Gefahren für die Schüler müssen in nächster Zukunft stark reduziert werden! Bei Ausgabe von Mahlzeiten für etwa $15 \%$ der Schüler verfügen einige Sportlehrkräfte schließlich auch über ein eigenes Budget (um 20\%). Aktuell dringliche Anschaffungen können also nur von weniger als einem Viertel der Lehrerinnen und Lehrer getätigt werden. Insgesamt besteht hier dringender Handlungsbedarf, der einerseits von den Sportlehrkräften und andererseits von der Administration zu lösen wäre.

\begin{tabular}{|c|c|c|c|c|c|}
\hline \multirow{3}{*}{ Item } & \multirow{3}{*}{$\begin{array}{l}\text { Antwort- } \\
\text { möglichkeiten }\end{array}$} & \multicolumn{2}{|c|}{ Mittelschule } & \multicolumn{2}{|c|}{ Oberschule } \\
\hline & & Sportlehrer & $\begin{array}{l}\text { Sportlehre- } \\
\text { rinnen }\end{array}$ & Sportlehrer & $\begin{array}{c}\text { Sportlehre- } \\
\text { rinnen }\end{array}$ \\
\hline & & \multicolumn{2}{|c|}{ Häufigkeiten in \% } & \multicolumn{2}{|c|}{ Häufigkeiten in \% } \\
\hline \multirow{17}{*}{$\begin{array}{l}\text { 89- Wel- } \\
\text { che } \\
\text { Hilfsmittel } \\
\text { liegen für } \\
\text { Ihren } \\
\text { Sportun- } \\
\text { terricht } \\
\text { tatsäch- } \\
\text { lich vor? }\end{array}$} & Turnmatten & 21.9 & 477.1 & 33.0 & 28.5 \\
\hline & \begin{tabular}{|l|} 
Fußbälle/Handbälle/ \\
Basketbälle/Volleybälle
\end{tabular} & 75.0 & 76.5 & 79.6 & 81.8 \\
\hline & Ersatzbälle & 66.3 & 47.1 & 68.5 & 54.5 \\
\hline & \begin{tabular}{|l|} 
Medizinbälle \\
\end{tabular} & 30.8 & 41.2 & 37.0 & 45.5 \\
\hline & \begin{tabular}{|l|} 
Hürden \\
\end{tabular} & 50.0 & 23.5 & 35.2 & 27.3 \\
\hline & Springseile & 22.1 & 23.5 & 33.3 & - \\
\hline & Gymnastikbänke & 51.0 & 82.4 & 57.4 & 81.8 \\
\hline & \begin{tabular}{|l|} 
Kasten/Pferd \\
\end{tabular} & 36.5 & 23.2 & 43.9 & 31.4 \\
\hline & Keulen/Reifen/Stäbe & 44.2 & 29.4 & 38.9 & 19.7 \\
\hline & Fußballtore & 85.6 & 23.5 & 83.2 & 27.3 \\
\hline & \begin{tabular}{|l|} 
Handballtore \\
\end{tabular} & 89.4 & 88.2 & 85.3 & 93.9 \\
\hline & Basketballkörbe & 87.5 & 82.4 & 70.4 & 68.5 \\
\hline & $\begin{array}{l}\text { Volleyballnetz incl. } \\
\text { Pfosten }\end{array}$ & 78.8 & 88.2 & 85.2 & 90.9 \\
\hline & Kugel (Kugelstoß) & 27.9 & 47.1 & 44.4 & 81.8 \\
\hline & Diskus/Speer & 22.1 & 35.3 & 25.9 & 18.2 \\
\hline & Sandgrube & 22.1 & 29.4 & 20.4 & 45.5 \\
\hline & \begin{tabular}{|l} 
Lange Maßbänder \\
\end{tabular} & 43.3 & 35.3 & 40.7 & 89.2 \\
\hline
\end{tabular}

Tabelle 76: Bereitstehende Hilfsmittel.

Aus eigenen Beobachtungen verfügen die meisten Sportlehrkräfte in Ägypten über eine Armbanduhr mit Stoppuhr. Im Hinblick auf Bewertungsmethoden wurde letztere daher nicht erhoben. Diesbezüglich fällt aber auf, dass nur in den Oberschulen für die Mädchen eine befriedigende Anzahl von Maßbändern vorliegt. Sonst 
fehlen für die gewünschten (und notwendigen) Bewertungen beispielsweise in Wurfdisziplinen oder für das Markieren der Sportstätten in mehr als der Hälfte der Fälle Maßbänder. In Bezug auf verschiedene Sportarten ergab sich folgendes Bild:

Für das Turnen liegen nur zu durchschnittlich einem Drittel Turnmatten vor. Sie wären weiterhin nicht nur zur Sicherheit der Schüler hilfreich, sondern auch für die gymnastischen Übungen oder bei der Vermittlung von Fertigkeiten einsetzbar. Über Gymnastikbänke verfügen 50\% der Jungenschulen und 80\% der Mädchenschulen. Möglicherweise steht dahinter der Gedanke, diese als Schwebebalken einzusetzen. Sie sind aber nicht nur aus organisatorischen Gründen hilfreich, sondern ebenfalls zur Fähigkeits- und Fertigkeitsschulung einsetzbar. Letzteres gilt auch für Pferde/Kästen (wobei das Pferd als Teildisziplin im Lehrplan gefordert ist) und für gewissermaßen klassische Hilfsmittel wie Seile, Medizinbälle, Stäbe usw. Weil diese lediglich zu 20-40\% vorhanden sind, scheint eine gezielt unterstützte und differenzierte Vermittlung sportartspezifischer Fertigkeiten sowie eine variantenreiche Fähigkeitsvermittlung in den überwiegende Fällen zumindest deutlich erschwert oder gar unmöglich.

Für die Spielsportarten sind die wesentlichen Bedingungen (Bälle und Pumpe, Tore usw.) durchgehend zu einem großen Teil vorhanden. Vielfach liegen sogar Ersatzbälle vor. Auffällig ist hier, dass Frauen kaum über Fußballtore verfügen. Vielfach liegen die Werte aber trotzdem häufiger um $75 \%$, was bedeutet, dass etwa ein Viertel nicht über diese zentralen Bedingungen verfügt. Ein weiteres Mal wird deutlich, welche wichtigen Aufgaben die Administration zu bewältigen hat, um eine flächendeckende Versorgung zur Umsetzung des Lehrplans zu erwirken.

Auch für die technischen Disziplinen der Leichtathletik (Würfe, Stoß, Hürden, Weitsprung) liegen die Bedingungen im Durchschnitt nur zu 20-30\% vor, sodass zu vermuten ist, dass hier eher Laufdisziplinen durchgeführt werden.

Insgesamt sind kaum systematische Unterschiede zwischen Geschlechtern und Schulformen auszumachen, wobei nach den Daten Jungenschulen etwas besser mit Fußballtoren, Hürden sowie Keulen/Reifen/Stäben und Mädchenschulen besser mit Medizinbällen und Gymnastikbänken ausgestattet sind. Viele klassische Hilfsmittel für einen abwechslungsreichen und in kleine Lernschritte zerlegten Sportunterricht fehlen in der überwiegenden Anzahl der Fälle. Zwar sind die zentralen Bedingungen zur Ausübung der Spielsportarten und für einfach durchzufüh- 
rende Teildisziplinen der Individualsportarten (wie Laufen oder Bodenturnen) gegeben, aber kaum die Grundlagen für die weitergehende Vermittlung spezieller Techniken, wie es im Lehrplan vorgesehen ist.

Zusammenfassend ist zu den Rahmenbedingungen und Hilfsmittel festzustellen, dass sie in nur unbefriedigendem Ausmaß gegeben sind. Hauptsächlich fällt dies in den Arbeitsbereich der Administration, wobei als zentrale Aufgaben folgende angesehen werden können: 1. Schaffen von Sanitäranlagen und Umkleideräumen, 2. Verbesserung der Bedingungen, um einzelne Sportarten überhaupt ausüben und bewerten zu können oder Überdenken des Lehrplanes, 3. Bereitstellung von Hilfsmitteln für die Durchführung von Turnen/Gymnastik, wobei diese gezielt bei der Vermittlung von Fertigkeiten in den anderen Sportarten eingesetzt werden könnten. Daneben muss aber auch erreicht werden, dass sich die Sportlehrkräfte stärker um die Notfallversorgung kümmern.

\subsubsection{Zusammenfassende Diskussion}

Obwohl im Gegensatz zu den Meinungen der Sportlehrerinnen und Sportlehrer innerhalb der Praxis des Sportunterrichts teilweise stärkere Unterschiede zeigten, scheint dies nicht immer erwähnenswert. Sie werden daher nur in den Fällen genannt, die vor dem Hintergrund der Strukturanalyse bedeutsame Schlussfolgerungen ermöglichen. Ein weiterer Unterschied zum Untersuchungskomplex ,Meinungen' liegt darin, dass geringe Zustimmungen bzw. ,Nein'-Antworten nicht schwerer ins Gewicht fallen, denn diese sind (vom Untersucher) eher überprüfbar (siehe Kapitel 5.1.4). Ein letzter Unterschied besteht darin, dass die Befunde vielfach schon für sich sprechen und weniger Interpretationen erfordern. Zu den drei Kategorien werden daher hier vielfach nochmals Ergebnisse wiedergegeben:

Die Umsetzung der Erziehungsziele erbrachte vielfältige Einzelbefunde, die aber erst abschließend in einen zusammenfassenden Rahmen gegossen werden sollen. Zuvor werden die wichtigsten Einzelergebnisse der Unterkategorien wiederzugeben. 
In Bezug auf das Erziehungsziel Freizeit wurde deutlich, dass nur etwa ein Viertel der Sportlehrkräfte gezielt daran arbeitet und kaum Talentsichtungen vorgenommen werden. Unter Berücksichtigung der gewünschten Förderung des Schulsports ist damit kaum eine entsprechende Gestaltung zu erkennen. Obwohl Sportlehrer insbesondere Fußball, Sportlehrerinnen ein breiteres Spektrum an Sportarten und Oberschullehrkräfte besonders Leichtathletik fördern, bleibt es (in nur wenigen Fällen) bei allgemeinen Einladungen und einer fehlenden Förderung selbständigen Sporttreibens.

Gesundheitliche Kenntnisse werden ebenfalls eher in allgemeiner Form vermittelt, dies aber mehr in der Mittelschule. Der Schwerpunkt liegt in diesem Erziehungskomplex eher auf Verletzungsprophylaxe und dem „Trainingskonzept der Gesundheitserziehung“, die in der Oberschule stärker akzentuiert erscheinen.

Im Hinblick auf Sozialverhalten wurde festgestellt, dass Konfliktlösungen fast immer durch die Lehrer vorgenommen werden - und dies ausgeprägter bei den Sportlehrerinnen und den Lehrkräften der Oberschule. Während das Lernen von ,Zusammenarbeit' auf den Aufbau der Gerätschaften für den Sportunterricht, auf Aufstellungsformen bei der Gymnastik und auf das taktische Zusammenspiel in den Mannschaftssportarten bezogen wird, beziehen die Sportlehrkräfte Akzeptanz auf gute Schüler. Wahrscheinlich werden kaum Gruppenarbeiten durchgeführt und die Annahme, dass sich im ägyptischen Sportunterricht eine stärker hierarchisierte Rollenbildung wieder findet, kann als bestätigt angesehen werden.

Die Ergebnisse zur Unterkategorie psychische Eigenschaften lassen eine Erziehung hin zur Konformität und eine deutliche Tendenz dahingehend erkennen, Kampfgeist und Selbstvertrauen durch Wettkämpfe zu fördern. Dies dient aber nur den sehr guten Schülern. Nur für sie sind solche Bedingungen nicht schlecht.

Bei einer überwiegenden Vermittlung von Regelkenntnissen werden taktische Kenntnisse praktisch nicht vermittelt (Unterkategorie sportspezifische Kenntnisse). Dies rückt auch den obigen Aspekt Zusammenarbeit in ein weniger günstiges Licht, wobei kaum vielfältige Lernerfahrungen ermöglicht werden.

Die Vermittlung von Freude / Spaß / Begeisterung findet in den Sportarten zu weniger als $50 \%$ statt. Wenngleich Frauen etwas mehr Hilfestellungen in vielen Sportarten bieten, findet eine rigide Unterrichtsstrukturierung bei einer geringen Berücksichtigung der Bedürfnisse statt. Allerdings werden vielfach Rückmeldungen gegeben und gute Schüler finden wiederum Anerkennung. Daraus ist zu 
schließen, dass gute Schüler wohl eher Spaß am Sport finden, wobei die wahrscheinlich freudvollsten Sportarten Fußball, Basketball, Volleyball und Leichtathletik sind.

Zusammenfassend ist festzustellen, dass eine deutliche Erziehung zur Konformität stattfindet. Die Schüler sollen gehorchen. Dies spiegelt letztlich die Entstehungsgeschichte des ägyptischen Sportunterrichts (das „Britische System“ mit Soldaten als Sportlehrer) und die Kulturinhalte des Islam wider, wobei interessant ist, dass dies bei den Sportlehrerinnen ausgeprägter ist. Während kaum selbständiges Handeln der Schüler unterstützt wird, haben es sportlich geschickte Schüler leicht in diesem System, werden aber trotzdem wenig gezielt gefördert. Letztlich wird von den Sportlehrern nur das Notwendigste getan und es findet bei den Sportlehrerinnen eine Konzentration auf die Sportarten Basketball, Volleyball und Leichtathletik und bei den Männern einseitig auf Fußball und mitunter auf Leichtathletik statt. Während die Erziehungsziele Freizeit, Sozialverhalten und sportspezifische Kenntnisse kaum und wenig leistungsförderlich umgesetzt werden, findet eine Vermittlung der Erziehungsziele Gesundheit, psychische Eigenschaften und Freude/Spaß statt, wenngleich auch nur in allgemeiner Form. Bei den drei ersten Erziehungszielen besteht also ein starker Integrationsbedarf und bei den letzten Dreien ein Nachholbedarf i.S. von Spezifikationen.

Auch innerhalb der Praxis des Sportunterrichts spiegeln die Ergebnisse zum methodischen Vorgehen teilweise wieder die Befunde zu den Erziehungszielen. Beispielsweise haben sich Wettkämpfe als wichtigstes Mittel der allgemeinen Methodik herausgestellt. Das weniger wichtige (aber in einer Rangfolge auf Platz 2 liegende) Mittel ,Rückmeldungen' wird nur noch von der Hälfte der Sportlehrer in ihrem Unterricht eingesetzt. Gezielte Verbesserungen sind also noch nicht das wichtigste Anliegen der Sportlehrkräfte, jedoch wird die Gymnastik streng nach Lehrplan vermittelt. Daneben sind die Lehrer häufig dort ein Vorbild, wo sie auch die Übungen vormachen. Schließlich finden kaum Prüfungsvorbereitungen statt, wodurch ein gezieltes Arbeiten weiter unterstützt werden könnte.

Beim Einsatz kollektiver Methoden werden meist einzelne sportspezifische Methoden eingesetzt. Die Frauen setzen aber häufiger auch ein Methodenmix ein, wobei nicht unbedingt gefolgert werden kann, dass sich der Einsatz an den Lernerfordernissen der Schüler orientiert. Bei den Männern ist dies sogar unwahrscheinlich. 
Bei dem Versuch, inhaltliche Schwerpunkte zu ermitteln, stellte sich allerdings umgekehrt heraus, dass im Hinblick auf Fähigkeiten kaum einseitig vorgegangen wird. Allerdings ist bei den männlichen Lehrkräften mit der leichten Bevorzugung von Ausdauer wieder die Tendenz zum Trainingskonzept Gesundheit erkennbar. Interessanterweise spielen die Grundfertigkeiten in den Oberschulen eine gleichberechtigte Rolle wie die speziellen Fertigkeiten, obwohl die Schüler hier doch eher Letztere lernen sollten. Hier zeigt sich wahrscheinlich ein gering ausgeprägter Leistungsstand, der seine Ursache vermutlich in der wenig ausdifferenzierten Methodik hat.

Schließlich bewerten nur etwa die Hälfte der Sportlehrkräfte ihre Schüler. Die Männer meist durch Endbewertung und die Frauen stärker durch Zwischenbewertung.

Die schon in der Einleitung getroffene Aussage (schlechte materielle Ausstattung) konnte durch die Erhebung zu den Rahmenbedingungen und Hilfsmitteln empirisch untermauert werden. Vielfach kann der Lehrplan deshalb gar nicht umgesetzt werden. Insofern ergeben sich für die Administration neben einer möglichen Adaption des Lehrplanes vier zentrale Aufgaben: 1. Bereitstellung von Hilfsmitteln für Turnen und Gymnastik, weil diese auch für einen variantenreichen Unterricht insbesondere bei der Fertigkeitsvermittlung - hilfreich wären, 2. Überprüfung und Optimierung der Notfallversorgung sowie Schaffung von Sanitäranlagen zur Förderung der Gesundheit, 3. Herstellen der Bedingungen für die Bewertung und Pflege der Sportstätten und 4. Schaffen von Umkleideräumen.

Resümierend seien folgende Punkte herausgehoben:

1. Das Erziehungsziel Gesundheit wird mit dem Trainingskonzept, psychische Eigenschaften mit Wettkämpfen und Freude/Spaß/Begeisterung in erster Linie mit den Sportarten Fußball und Leichtathletik bei den Männern und Basketball, Volleyball und Leichtathletik bei den Frauen gefördert. Hier besteht Erweiterungsbedarf.

2. Konformität und eine geringe Betonung von Selbständigkeit stehen im Vordergrund der sozialen Umgangsformen. Eine stärkere Berücksichtigung der Erziehungsziele Freizeit (insbesondere Talentsichtung), Sozialverhalten (v.a. Förde- 
rung von Selbständigkeit und schwächerer Schüler) und taktischer Aspekte scheint angezeigt. Dies trifft stärker auf die Sportlehrerinnen zu.

3. Rückmeldungen und Methoden für große Klassen und einzelne sportspezifische Methoden sind Schwerpunkte beim Unterrichten. Ihr differenzierter Einsatz und ihre Wechselwirkungen müssen allerdings stärker in die Ausbildung eingehen (mehr noch bei den Männern als bei den Frauen). Gleiches gilt für Bewertungen.

4. Rahmenbedingungen und Hilfsmittel liegen in nur sehr unbefriedigendem Ausmaß vor. Hier ist die Administration gefordert - entweder mit einer Adaption des Lehrplans oder mit einer konkreten Lösung der dringendsten Probleme.

\subsection{Vergleichende Interpretation}

Die zwei großen Untersuchungsaspekte (Meinungen der Sportlehrerinnen und Sportlehrer und die Praxis des Sportunterrichts) seien an dieser Stelle zunächst aufeinander bezogen. In einem zweiten Schritt soll der zusammenfassende Vergleich aber auch mit den Ergebnissen der Strukturanalyse konfrontiert werden. Insgesamt zielt dies darauf ab, die Bedingungen seitens der Sportlehrkräfte und in Bezug auf die Kategorien Erziehungsziele, methodisches Vorgehen und Rahmenbedingungen / Hilfsmittel zu klären, um darauf aufbauend Aussagen zur Optimierung des Systems treffen zu können. Vorwegnehmend sei dazu festgestellt, dass praktisch keine systematischen Unterschiede zwischen den Schulformen auszumachen waren.

Die Fragestellung, ob die übergreifenden Erziehungsziele von den Sportlehrern akzeptiert und in ihrem Unterricht berücksichtigt werden, kann wie folgt beantwortet werden:

Die Erziehungsziele lassen sich in zwei Gruppen einteilen. Die erste Gruppe, zu denen Gesundheit, psychische Eigenschaften und Freude/Spaß/Begeisterung gehören, wird von den Sportlehrkräften sehr hoch bewertet bzw. diese Erziehungsziele werden klar akzeptiert. Sie spiegeln den Charakter des Sporttreibens wider, wobei hier ein leistungssportliches Verständnis der Lehrerinnen und Lehrer er- 
kennbar wird. Diese Ziele werden auch in der Praxis verfolgt, wenngleich nur in allgemeiner Form: Die Verfolgung des Erziehungszieles Gesundheit wird eher mit dem "Trainingskonzept" (und weniger einer Wissensvermittlung) vollzogen, die Förderung psychischer Eigenschaften erfolgt durch Wettkämpfe und der (gewollte) Unterricht zeigt eine starke Erziehung zur Konformität mit einer besonderen Förderung leistungsstarker Schüler. Aufgrund nur wenig ausdifferenzierter Maßnahmen ergibt sich in dieser Gruppe die Erziehung also eher nebenbei, wobei Sportlehrerinnen dabei stärker Hilfestellungen und Rückmeldung geben wollen und geben, jedoch geringere Ausprägungen beim Erziehungsaspekt Führungsqualität und beim Vorbild-Sein aufweisen.

Die zweite Gruppe der Erziehungsziele (Freizeit, Sozialverhalten, sportspezifische Kenntnisse) wird deutlich geringer bewertet. Dabei finden sich v.a. die ablehnenden Haltungen bei der Untersuchung der Praxis wieder. Festzuhalten ist, dass sich erneut eine Neigung zu konformistischer Erziehung mit einem eher autoritären Führungsstil zeigt und praktisch keine Förderung selbständigen Handelns (der Schüler) stattfindet. Daneben werden trotz ausgeprägter Anerkennung guter Schüler nur wenig Talentsichtung vorgenommen; im Erziehungsaspekt Sozialverhalten scheint nur eine allgemeine und vornehmlich auf die Gymnastik bezogene Zusammenarbeit stattzufinden - bei meist fehlender Vermittlung taktischer Inhalte in den Mannschaftssportarten. Ursachen für derartige Befunde lassen sich einerseits in den großen Klassen finden, andererseits im kulturellen Hintergrund, wobei Frauen die Ausprägungen (sowohl Ablehnung als auch Zustimmung) stärker zeigen.

Insgesamt sind die Ergebnisse zu den Meinungen und die Umsetzung in der Praxis also kongruent. Sportarten werden aufgrund der Befunde wahrscheinlich schwerpunktmäßig durchgeführt ${ }^{23}$ und die Erziehungsziele der ersten Gruppe hier auch umgesetzt. Defizite für derartige Verhältnisse sind vornehmlich in der Ausbildung zu suchen. Daher ist zu fordern, dass im Studium insbesondere eine stärkere Integration psycho-sozialer Aspekte erfolgen sowie eine stärkere Spezifizierung gesundheitlicher und taktischer Kenntnisse stattfinden sollte.

${ }^{23}$ Frauen widmen sich stärker dem Basketball, dem Volleyball und der Leichtathletik, während Männer sich einseitig auf Fußball „eingeschossen“ haben, aber auch Leichtathletik stärker berücksichtigen. 
Das gewünschte und tatsächlich durchgeführte inhaltliche und methodische Vorgehen der Sportlehrerinnen und Sportlehrer lässt sich folgendermaßen beschreiben:

Die Befunde zu den Erziehungszielen sind vielfach wieder zu finden. Auch die Methodik zielt in der Meinung der Sportlehrkräfte auf Leistungssport und Wettkämpfe sind das Mittel der Wahl. Erkennbar wird noch deutlicher als bei den Erziehungszielen, dass Frauen stärker gesellschaftlichen Konventionen folgen (wollen).

Allerdings weichen die Meinungen teilweise auch von der Umsetzung in der Praxis ab. Bei meist hoher Zustimmung von grundlegenden Methoden wie Rückmeldungen (positiv, richtig/falsch), Anleitungen oder Vormachen werden sie zwar meist, jedoch häufig nur von der Hälfte der Sportlehrerlnnen angewandt. Ähnliches gilt für die Bewertung, die zwar (als Teil der Ausbildung) als wichtig beurteilt, in der Praxis aber nur unzureichend und wenig systematisch umgesetzt wird. Dies liegt wahrscheinlich an der fehlenden Sportnote im Zeugnis.

Demgegenüber wird die Förderung selbständigen Handelns der Schüler häufiger abgelehnt und praktisch nicht verfolgt; individuelle Methoden werden nur zum Teil als wichtig erkannt und praktisch nicht angewandt.

Weil zudem nur herausragende Leistungen belohnt werden, wird erkennbar, dass insbesondere leistungsschwache Schüler kaum Unterstützung finden. Dies kommt auch an der inhaltlichen Orientierung der Lehrer (in Meinung und Praxis) zum Ausdruck: Auch in der Oberschule werden zu gleichen Teilen Grundfertigkeiten und sportspezifische Fertigkeiten vermittelt, was die vielfach aufgetauchten Defizite bei der Vermittlung deutlich werden lässt. Sie erfolgen zwar mit den drei großen Methoden, jedoch findet häufig keine differenzierte Ausarbeitung und variable Anwendung dieser Methoden statt, was sich bei Frauen nicht so deutlich zeigt. Ein besonders wichtiges Thema, das in der Ausbildung stärker akzentuiert werden muss, ist eben diese differenzierte Anwendung von Methoden, welche inhaltlich vor dem Hintergrund der Klassenstärken mit Möglichkeiten individueller Korrekturen versehen werden sollten.

Die Erhebung der Lernvoraussetzungen (Rahmenbedingungen und Hilfsmittel) erbrachte folgende Befunde:

Bei einer problematischen Situation - Rahmenbedingungen und Hilfsmittel liegen vielfach gar nicht vor, sodass auch die Erziehungsziele häufig nicht umgesetzt 
werden können - ergeben sich für die Administration zwei Möglichkeiten. Entweder der Lehrplan wird verändert oder die Infrastruktur verbessert. In Bezug auf eine Verbesserung, d.h. wenn der Lehrplan nicht verändert werden soll, ergeben sich vier Aufgaben: a) Anschaffung von Hilfsmitteln für Turnen und Gymnastik, weil diese auch für einen inhaltlich und methodisch variantenreichen Unterricht - insbesondere bei der Fertigkeitsvermittlung - hilfreich wären, b) Optimierung der Notfallversorgung (möglicherweise durch die Bezirksleiter) sowie Bauen von Sanitäranlagen zur Förderung der Gesundheit, c) Herstellen der Bedingungen für die Bewertung und Pflege der Sportstätten (sowohl materiell und organisatorisch als auch im Hinblick auf Zeugnisnoten und Überprüfungen der Sportlehrer) und möglicherweise d) Schaffen von Umkleideräumen.

Berücksichtigt man zu diesen Befunden die Ergebnisse der Strukturanalyse (zusammenfassend Kapitel 2.5 und 3.6), so lassen sich folgende Schlussfolgerungen ziehen:

Während Islam und „Britisches System“ über Gehorchen, Konformität und eine fehlende Unterstützung der Selbständigkeit der Schüler im Sportunterricht erkennbar sind, spielen nicht sie die wichtigste Rolle, sondern das öffentliche System des Sports bzw. die Sportstruktur. Fußball hat als Sportart eine herausragend hohe Bedeutung in Ägypten, ist aber eine Männersportart. Deswegen sind auch die diesbezüglich hohen Werte bei den männlichen Lehrkräften erklärbar. Im Sportunterricht wird Fußball vermutlich häufig ausgeübt (denn die Sportlehrer können die Inhalte akzentuieren) und die Erziehungsziele der ersten Gruppe werden daher wahrscheinlich auch hier umgesetzt bzw. hineingelegt. Sportlehrerinnen orientieren sich an einem breiteren Spektrum, neigen aber auch zu den in Ägypten traditionell vermehrt ausgeübten Sportarten (vgl. Tab. 1 und 2). Turnen wird nur wenig ausgeübt - auch aufgrund mangelnder Bedingungen - und vielleicht sehen viele die Inhalte schon mit Gymnastik abgedeckt. Letztere dient vornehmlich der körperlicher Ertüchtigung (bzw. dem Trainingskonzept der Gesundheit i.S. von physischer Leistungssteigerung), wobei dies wahrscheinlich auch bei Fußball und Leichtathletik verfolgt wird.

Die vermittelten Erziehungsziele in den schwerpunktmäßig gelehrten Sportarten lassen sich aber auch aus der Wettkampfstruktur des Schulsports interpretieren (siehe S. 43). So ist es vor diesem Hintergrund (und der Beurteilung der Erfolge 
der Sportlehrer durch die Bezirksleiter) nicht verwunderlich, dass sie insgesamt leistungssportlich verstanden werden und dass vielfach Wettkämpfe auch im Sportunterricht erfolgen. Dabei unterstützt sicherlich auch der Aspekt Konformität. Umgekehrt wurde aber festgestellt, dass kaum gezielte Maßnahmen getroffen und selbst grundlegende methodische Vorgehensweisen nur unzureichend umgesetzt werden. Eine gezielte Orientierung auf systematische Leistungsverbesserungen findet bei schlecht ausgestatteten Sportstätten kaum statt. Die nur relativ geringe Umsetzung des Erziehungszieles Freizeit erscheint dabei zunächst unverständlich, denn eine systematische Talentsichtung findet nicht statt. Daher ist zu vermuten, dass die meisten Schüler ein nur sehr niedriges Niveau in den verschiedenen Sportarten aufweisen, was seine Ursache wiederum in der mangelhaften Methodik hat.

Aus diesen Ergebnissen kommt für die Zukunft zunächst folgende mögliche Perspektive in betracht. Es könnte eine Revision des Lehrplanes vorgenommen werden. Vielleicht wäre es für die Entwicklung der ägyptischen Sportstruktur hier vorteilhaft, im Sportunterricht eine breitere Palette an Sportarten festzuschreiben, diese aber dafür umgekehrt weniger spezifisch zu vermitteln. Damit würde eine bessere allgemeine sportliche Ausbildung der ägyptischen Bevölkerung möglich. In diesem Zuge müsste aber auch eine stärkere Trennung von Sportunterricht und Schulsport stattfinden. Letzterer sollte dann umgekehrt für ein gezieltes Leistungstraining stehen, in dem Leistungssteuerung (incl. -kontrolle, s.u.) eine wesentliche Rolle spielt. Also: Im Sportunterricht etwas weg von der Leistungsorientierung, damit die Erziehungsziele insgesamt stärker umgesetzt werden, dafür im Schulsport eine stärkere Hinwendung. Dies könnte auch dazu beitragen, dass Eltern ihre Kinder - sofern sie talentiert sind - eher zum Schulsport schicken, denn hier ergibt sich dann eine klare Zielformulierung. Weil aber für einen solchen Schulsport eine weitergehende Methodenausbildung notwendig wäre und umgekehrt auch im Sportunterricht Methodendefizite zu erkennen sind, ist der Schwerpunkt der optimierenden Maßnahmen klar in dieser Richtung zu sehen. Ob obige Perspektive nun angegangen wird oder nicht: Die fruchtbarste Maßnahme liegt in einer differenzierten Methodenausbildung.

Auch wenn im Hinblick auf die Methoden auffällt, dass Sportlehrerinnen eine bessere Ausbildung als ihre männlichen Kollegen erhalten haben, weil sie mehr Rückmeldungen geben, eher ein Methodenmix anwenden und Zwischenbewer- 
tungen durchführen (sich umgekehrt aber stärker an Konventionen orientieren und weniger Vorbild sein wollen), besteht Nachholbedarf. Obwohl EL GOHARIE (2002) schon auf eine erste Entwicklung in diesem Bereich hinweist, müssen verschiedene Aspekte größere Bedeutung erlangen. Erfolgversprechend erscheint beispielsweise eine stärkere Verbindung zwischen Methode und Situation (bzw. Theorie und Praxis), um dies auch später leichter anwenden zu können. Weiterhin kann es fruchtbar sein, zu thematisieren, wie einzelne Methoden in unterschiedlichen bewegungsbezogenen Problemfeldern und bei unterschiedlichen Leistungsniveaus angewandt werden. Ein erster Schritt besteht jedoch in der Vermittlung variantenreicher Anwendungen der Ganz-Teil-Methode mit ihren unterschiedlichen Kombinationsmöglichkeiten und parallel dazu: der effektive Umgang mit großen Klassen. Möglicherweise ist es zur Umsetzung der Erziehungsziele auch hilfreich, in der Sportlehrerausbildung die Präsentation theoretischer Inhalte zu betonen. Dies wäre eine gute Voraussetzung dafür, dass auch bei schlechtem Wetter oder während des Ramadans ein gezielter Unterricht stattfinden kann, und zwar in Form von Theorieunterricht. Fruchtbare Themen im Hinblick auf die Erziehungsziele und die in der Praxis aufgedeckten Methodendefizite könnten z.B. sein: Gesundheit und Trainingslehre, Taktiken, Informationen über nationale und internationale Meisterschaften, und Umgang mit Konflikten.

Die genannten Empfehlungen für die Administration - v.a. in Bezug auf Rahmenbedingungen und Hilfsmittel - sind abschließend kritisch zu betrachten. Vor dem Hintergrund der volkswirtschaftlichen Situation scheint kaum Aussicht auf eine Umsetzung gegeben, weil vermutlich kaum monetäre Ressourcen für eine flächendeckende Versorgung der Schulen mit Rahmenbedingungen/Hilfsmitteln bereit gestellt sowie organisatorischen Maßnahmen getroffen werden können bzw. wollen. Dies betrifft auch eine weitere mögliche Maßnahme, nämlich die mögliche Teilung der Klassen, um stärker auf die einzelnen Schüler eingehen zu können. Dazu wären deutlich mehr Lehrkräfte notwendig, was entsprechend kostspielig erscheint. Daher sind insgesamt stärker die Ausbildung bzw. die Sportinstitute gefordert, die durch ihr Know-How ebenfalls zu einer weiteren Revision des Lehrplanes beitragen können. Dabei sind aber wegen der fehlenden Breitensportkultur kaum die Ziele und Strategien der mitteleuropäischen oder nordamerikanischen Länder zu übernehmen, sondern eigene Ansätze zu entwickeln, wie der folgende Ausblick zeigen soll. 


\section{Ausblick}

Im vorangegangenen Kapitel wurde deutlich, dass höchstens die Hälfte der Erziehungsziele im ägyptischen Sportunterricht umgesetzt wird und dies nur in allgemeiner Art. Die wichtigste Ursache dafür wurde in ausgeprägten Defiziten der Sportlehrerinnen und Sportlehrer beim methodischen Vorgehen gesehen. An dieser Stelle seien die Ergebnisse - unter Zuhilfenahme des deutschen Kenntnisstandes und unter Berücksichtigung der zuvor ausgearbeiteten Konsequenzen - in einen übergreifenden Rahmen gesetzt. Dies bedeutet nicht nur eine Reflexion der Ergebnisse, sondern auch Empfehlungen im Hinblick auf mögliche zukünftige Strategien der ägyptischen Sportpädagogik. Ausgehend von den „drei Säulen der Didaktik" (vgl. FAVRE, 1997), nämlich Lehrerausbildung, Forschung und Schulpolitik, scheint es aber ebenfalls notwendig, zusätzlich inhaltliche Aspekte mit aufzugreifen. Anhand der Beantwortung von vier Fragen wird nun ein gangbarer Weg für die ägyptische Sportpädagogik gezeichnet:

1. Wie sieht der Stand der Forschung aus, d.h. welche Wissensgrundlage besteht (auch unter Berücksichtigung dieser Arbeit) für Veränderungen?

2. Was bedeutet dies für die inhaltliche Gestaltung bzw. wie kann sie optimiert werden?

3. Was sollte daraufhin in der Lehrerausbildung passieren? und

4. Welche Konsequenzen ergeben sich für die Schulpolitik?

1. Die vorliegende Arbeit versuchte erstmals, den ägyptischen Sportunterricht anhand der Kategorien Erziehungsziele, methodisches Vorgehen und Rahmenbedingungen auszuleuchten. Dabei wurden Meinungen und die tatsächliche Umsetzung in die Praxis untersucht und anschließend konfrontiert. Die erhobenen Meinungen der Sportlehrerinnen und Sportlehrer können nun als relativ sichere Wissensbasis gelten, wobei die Zustimmungsneigung berücksichtigt werden muss. Wichtigstes übergreifendes Ergebnis scheint trotzdem, dass die Inhalte des Lehrplans sehr deutlich befürwortet werden, aber eher allgemeine Aspekte das Meinungsbild der Sportlehrer leiten. Vor dem Hintergrund der Ausbildung an den Sportinstituten muss gefolgert werden, dass auch hier nur 
eine eher allgemeine Wissensvermittlung stattfindet. Hier besteht also Nachholbedarf, wobei zu vermuten ist, dass dies auch für die Forschung gilt (wenig ausdifferenzierte Wissensbestände). Umgekehrt steht die Erhebung der Praxis noch nicht auf festen Beinen, obwohl erste gängige Muster aufgedeckt werden konnten. Zukünftig erscheint es daher hilfreich, Unterrichtsbeobachtungen vorzunehmen. Hier kommen Videos oder Protokolle in Frage und zwar zunächst unstrukturiert, um bedeutsame Kategorien aufzudecken, sowie (daran anschließend) strukturiert und bei größeren Stichproben.

Hilfreich für die Gesamtentwicklung des Faches wäre sicherlich die Ergänzung um eine weitere Perspektive. Verglichen mit den Positionen der deutschen Sportpädagogik (pädagogische Position mit Blick auf Bildung und Erziehung, unterrichtliche Position mit Blick auf Lehren und Lernen im Sport sowie sachbezogene Position mit Blick auf die Qualifizierung für den Sport; vgl. EHNI, 2000) sind die erste Position sowie psycho-soziale Aspekte in der ägyptischen Sportpädagogik kaum zu erkennen. Dies mag an dem kulturellen Hintergrund liegen, denn die Aufklärung (als philosophische Grundlage der Wissenschaft) hat in den (islamisch-)arabischen Staaten noch nicht eingesetzt. Um hier erste Impulse zu setzen, scheint ein stärkerer wechselseitiger Austausch von Wissenschaftlern zwischen Deutschland und Ägypten hilfreich.

2. Hinsichtlich der inhaltlichen Gestaltung (des Lehrplanes, siehe dazu beispielsweise Abb. 4) und vor dem Hintergrund des Erziehungszieles Gesundheit einerseits sowie eines effizienten Trainierens andererseits sollte ein Überdenken einsetzen. Als erste Wissensbasis für eine gesunde und effiziente Gymnastik lässt sich die „Funktionsgymnastik“ von KNEBEL (1985) heranziehen, die mittlerweile auch ins Russische übersetzt ist. Zusätzlich werden aber sicherlich auch Untersuchungen der ägyptischen Schüler notwendig. Durch trainingswissenschaftliche Fragestellungen, psychologische Fragestellungen (z.B. Motivation) und durch die Erhebung physischer Voraussetzungen können hier Grundlagen gelegt werden. Die resultierenden Maßnahmen sind dann weitergehend in Feldexperimenten zu überprüfen, beispielsweise durch Fitness- oder Motoriktests mit einem Pre-Post-Design.

Noch viel wichtiger scheint aber, verschiedene Methoden im Hinblick auf Leistungsniveaus, Altersgruppen und große Klassen auszudifferenzieren. Eine ers- 
te Orientierung dazu kann KURZ (1998) liefern. Anhand der folgenden zwei Beispiele sei aufgezeigt, wie sich dies schrittweise auf die Praxis übertragen lässt. Sie beziehen sich auf einen eher allgemein denn sportartspezifischen orientierten Unterricht, wie dies im vorhergehenden Kapitel angedacht wurde: MEUSELs (1993) Anliegen richtet sich auf eine Motivierung der Kinder zu vielfältiger körperlicher Bewegung. Dazu stellt er kleine Bewegungsspiele vor, die eine abwechslungsreiche und spannende Handlungssituation bieten. Anhand der Struktur des Buches (Ziele, Einsatz in Training, Unterricht und Freizeit, Bedeutung von Vorbereitung und Gelingen, Einbeziehen schwächerer Schüler sowie vielfältigen sich daraus ergebenden Möglichkeiten) könnte ein erster Leitfaden erstellt werden. Letzteres gilt ebenso für Ausführungen von RÖHRLE et al. (1997) im Hinblick auf die sportartübergreifende Vermittlung von Sportspielen. Weil auch in anderen Erziehungsaspekten Defizite zu verzeichnen waren, könnten derartige Übertragungen auch mit Ausführungen von PEUKE (1997) in Bezug auf soziales Lernen im Sport bzw. die Sportlehrerausbildung und mit KOTTMANN \& KÜPPER (1999) in Bezug auf Gesundheitserziehung stattfinden. Insgesamt heißt dies also: Übersetzung der Literatur ins Arabische, Studium und Adaption der Konzeptionen auf ägyptische Verhältnisse.

3. Die Sportlehrerausbildung muss mit obigen Überlegungen weg von solch allgemeinen Forderungen wie: „Die Vorbereitung fordert von ihm Kenntnisse aller Erziehungsverfahren, die er in seinen Vorbereitungsphasen auf die Lehrertätigkeit erlernt, sodass diese Verfahren ihm später bei Lösungen von Problemen im Schulalltag helfen“ (AZMY, 1996, 23). Eine erste Präzisierung könnte mit den Ausführungen von HOTZ (1995) erfolgen: Er argumentiert für ein bewusstes Erlernen und Wissen um Bewegungen in der Sportlehrerausbildung, um dadurch Demonstrations- und Instruktionsfähigkeit zu vermitteln. Sowohl Kennen (Theorie) als auch Können (Praxis) von Bewegungen wird als Grundlage eines effektiven „Bewegungsverstehens" zusammengesetzt, welches wiederum zum Kern seiner hochschuldidaktischen Konzeption wird. In Ägypten ist in dieser Hinsicht eher die theoretische Ausbildung gefordert (s.o.) mit einer sich möglichst anschließenden engen Verknüpfung in der Praxisausbildung.

4. In Abhängigkeit solcher Voraussetzungen und den Wünschen der Politik sollten dann die dringensten Probleme gelöst werden, damit die Schüler stärker 
zur Handlungsfähigkeit qualifiziert werden. Weil dies nicht nur die Schüler, sondern auch die Lehrer motivieren sollte, gehört dazu sicherlich die Einführung einer Zeugnisnote für das Unterrichtsfach Sport. Weitergehend muss zukünftig allerdings noch ausgehandelt werden, welche Erziehungsziele in erster Linie verfolgt werden sollen, denn der inhaltliche Ausbau der Sportlehrerausbildung kann kaum in alle Richtungen erfolgen. Entweder ergibt sich eine stärkere Orientierung auf die Aspekte Gesundheit, Sozialverhalten und Freizeit. Dann müsste man sich eher Gedanken um die Beziehung zum Sportsystem und um die Integration von Bildungs- denn Erziehungsinhalten machen. Oder der Leistungssport wird stärker gefördert. Dann sind Maßnahmen gefordert, die die Effektivität in der Leistungsentwicklung steigern.

Nichtsdestotrotz ist die Durchführung des Sportunterrichts zukünftig in den Mittelpunkt der Betrachtungen zu stellen. Dementsprechend sollten Lehrpläne weniger Äußerlichkeiten, sondern vielmehr methodisch-didaktische Rahmenkonzepte enthalten. Besonders wichtig scheinen hier Planungsüberlegungen, die sich einerseits auf die Unterrichtsplanung der Sportlehrer beziehen und andererseits auf administrative Maßnahmen. Dies ist aber nur sinnvoll, wenn deutlich wird, wie Erziehungsziele sich auf die Handlungsebene übertragen lassen (vgl. auch BECKER, 1998, 11), womit zuerst eine differenzierte sportdidaktische Konzeptionierung innerhalb der ägyptischen Sportwissenschaft geschaffen werden müsste.

Für Forschung und Lehre sind von der ägyptischen Sportpädagogik als wissenschaftliche Disziplin also vielfältige Aufgaben gefordert, wenn es ihr wie der deutschen Sportpädagogik um die Erfassung und Deutung des Geschehens im Sportunterricht geht, um dies besser verstehen und gestalten zu können (vgl. BRÄUTIGAM, 2003, 143). Insofern bietet diese Arbeit eine erste Orientierung für Veränderungen und eröffnet vielfältige Perspektiven vor dem Hintergrund der spezifischen kulturellen Bedingungen. 


\section{Zusammenfassung}

Die ägyptische Sportdidaktik hat mit drei bedeutsamen Problemen zu kämpfen, nämlich 1. die noch fehlende empirische Prüfung der Vermutung, dass die Sportstätten für den Sportunterricht schlecht ausgestattet sind, 2. die praktisch nicht erkennbare Verbindung zwischen übergreifenden Erziehungszielen und Lehrplänen sowie 3. die bisher ausgebliebenen Untersuchungen von Sportlehrerinnen und Sportlehrern. Ausgehend von diesen Wissenslücken versucht sich die vorliegende Arbeit in einer Analyse des ägyptischen Sportunterrichts, die als erste Exploration verstanden wird, um Perspektiven für zukünftige Forschung zu eröffnen. Gleichzeitig ist damit jedoch auch die Hoffnung verbunden, drängende Probleme aufzudecken und erste Lösungsstrategien bereit zu stellen.

Unter Zuhilfenahme eines Analyseschemas von SCHULZ (1972) wird deutlich, dass nicht nur die Feinstruktur des Unterrichts (Erziehungsziele, Inhalte und Methoden), sondern auch die Rahmenbedingungen sowie die sozial-kulturellen Voraussetzungen bedeutsame Bedingungen für den Sportunterricht darstellen. Obwohl sich der ägyptische Sportunterricht als Fach etabliert hat, muss er also in einen übergeordneten Zusammenhang gestellt werden, um Charakter und faktische Gegebenheiten darzustellen. Die Ergebnisse einer solchen Strukturanalyse, die bisher noch nicht erfolgt ist, lassen sich wie folgt zusammenfassen:

I. Während sich klimatische und volkswirtschaftliche Bedingungen als eher hinderlich für das Sporttreiben und den Sportunterricht darstellen, ist dies für den Islam als Staatsreligion und die familiären Bedingungen nicht unbedingt auszumachen. Das außerschulische Sportsystem ist klar leistungsorientiert und ermöglicht nur wohlhabenden Bürgern die Teilnahme am Vereinssport. Als populärste Sportarten konnten die Männersportart Fußball (mit einer starken Medienpräsenz) und andere Mannschaftssportarten sowie Leichtathletik und Kampfsportarten ausgemacht werden. Bei bestehender Schulpflicht wurde deutlich, dass sehr große Klassen vorliegen (über 40 Schüler), der Sportunterricht nicht mit einer Note im Zeugnis erscheint und zunächst genügend akademisch ausgebildete Sportlehrer vorhanden sind. Diese sind bei täglich 3-4 Unterrichtseinheiten und etwa 500 Schülern einer relativ hohen Arbeitsbelas- 
tung ausgesetzt; hinzu tritt der neben dem vormittäglichen Unterricht nachmittags stattfindende Schulsport, der sich in den populärsten Sportarten als Wettkampfsystem ebenfalls etabliert hat. Dortige Erfolge gelten dann als wichtigstes Kriterium für die Bezirksleiter (ehemalige Sportlehrer), um die aktuell unterrichtenden Sportlehrer für eine höhere Gehaltsstufe zu empfehlen.

II. Daraufhin wird der ägyptische Lehrplan (im Hinblick auf Erziehungsziele, Inhalte und Methoden) ausgeleuchtet, in dem aber nicht nur Vorschriften und Empfehlungen zum Sportunterricht, sondern auch Desiderate zum Schulsport und für die Sportlehrerausbildung formuliert sind. Neben nur sehr allgemeinen Aussagen zu persönlichen Eigenschaften des Sportlehrers sind auch die Erziehungsziele nur sehr allgemein formuliert, sodass letztere vor dem Hintergrund der ägyptischen (und wo nötig auch der deutschen) Sportpädagogik reflektiert werden. Zwar wird daraus auch ein mehrperspektivischer Unterricht (vgl. KURZ 1999) erkennbar, jedoch beziehen sich die Sinnebenen mit einem Wunsch nach Gesundheit stärker auf die Qualifizierung zum Schulsport und nur marginal auf gesellschaftliche Aspekte. Für die beabsichtigte nachfolgende Untersuchung wurden die Erziehungsziele in einzelne Unterkategorien zerlegt: Freizeit (motorische Voraussetzungen, Organisationsfähigkeit, verschiedene Sportarten), Gesundheit (Kenntnisse), Sozialverhalten (Konfliktlösung, Zusammenarbeit, Akzeptanz), psychische Eigenschaften (Selbstvertrauen, Kampfgeist, Führungsqualitäten, Gehorsam), sportspezifische Kenntnisse (Regeln, Sportereignisse, vielfältige Erfahrungen) und Freude / Spaß / Begeisterung (Rückmeldung, Hilfe, Bedürfnisse, Vorbild). Während die Inhalte des Sportunterrichts klar einer Vermittlung sportspezifischer Fertigkeiten in 7 Sportarten folgen, wird die einzelne Unterrichtseinheit streng strukturiert: Organisatorische Aufgaben und Aufwärmen, Schulung konditioneller Fähigkeiten mittels Gymnastik, Schulung sportartspezifischer Fertigkeiten, Schlussteil. Daneben wird die Bewertung als erforderlich erachtet, eine konkrete Übungsauswahl bereitgestellt und inhaltliche wie zahlenmäßige Angaben zu den Rahmenbedingungen/Hilfsmitteln getätigt. Die 8-semestrige Sportlehrerausbildung umfasst theoretische und fachpraktische Inhalte zu gleichen Teilen, wobei die Förderung der Persönlichkeit nur implizit erfolgt. So sollen unterschiedliche Teilgebiete der Sportwissenschaft und verschiedene Sportarten kennen gelernt werden, wobei der Schwerpunkt auf der Methodenausbildung nach all- 
gemeinen Prinzipien liegt. Zusätzlich werden mehrere Praktika in den Schulen absolviert.

Die einzelnen gewünschten Aspekte des Lehrplans wurden dann empirisch geprüft. Dazu wurde eine Befragung von 186 Sportlehrkräften in El Dakahlia/Ägypten durchgeführt. Sie orientierte sich an drei Leitfragen (Werden die übergreifenden Erziehungsziele von den Sportlehrern akzeptiert und im Unterricht berücksichtigt? Was wollen die Sportlehrer inhaltlich und methodisch in ihrem Unterricht unternehmen und was machen sie tatsächlich? Welche Lernvoraussetzungen sind gegeben, welche sind möglichst noch zu schaffen?), die in Bezug auf das Meinungsbild und die Praxis der Sportlehrer (Orientierung) folgende zentralen Ergebnisse zutage förderten.

4. Mittel- und Oberschullehrer unterscheiden sich kaum, jedoch orientieren sich Sportlehrerinnen etwas stärker als Sportlehrer an gesellschaftlichen Konventionen und erkennen Problemlagen etwas deutlicher.

5. Erziehungsziele, die mit freudvollem Leistungssport/Trainieren verbunden sind (Gesundheit, psychische Eigenschaften, Freude/Spaß), werden höher gewichtet als die Förderung des Schulsports (Freizeit), sportspezifische Kenntnisse und Sozialverhalten. Das Erziehungsziel Gesundheit wird mit dem Trainingskonzept, psychische Eigenschaften mit Wettkämpfen und Freude/Spaß werden in erster Linie mit den Sportarten Fußball und Leichtathletik bei den Männern und Basketball, Volleyball und Leichtathletik bei den Frauen in allgemeiner Art gefördert. Hier besteht Spezifikations- und Erweiterungsbedarf. Konformität und eine geringe Betonung von Selbständigkeit stehen im Vordergrund der sozialen Umgangsformen, sodass letztlich eine stärkere Berücksichtigung von Talentsichtung, der Förderung von Selbständigkeit und schwächerer Schüler sowie taktischer Aspekte angezeigt scheint. Dies trifft stärker auf die Sportlehrerinnen zu. Die vermittelten Erziehungsziele in den schwerpunktmäßig gelehrten Sportarten lassen sich aus der Wettkampfstruktur des Schulsports interpretieren. So ist es vor diesem Hintergrund (und der Beurteilung der Erfolge der Sportlehrer durch die Bezirksleiter) nicht verwunderlich, dass sie insgesamt leistungssportlich verstanden werden und dass vielfach auch Wettkämpfe im Sportunterricht erfolgen. 
6. Eine stärkere Berücksichtigung von Rückmeldungen und Hilfestellungen im Sportunterricht und von spezifischen Methoden (sportartspezifische Vermittlung und Bewertung) für die Ausbildung scheint empfehlenswert. Dies betrifft neben Konfliktlösungen selbständiges Lernen und Handeln der SchülerInnen, die variantenreiche Anwendung der Ganz-Teil-Methode und den effektiven Umgang mit großen Klassen. Obwohl anders vorgesehen, werden auch in der Oberschule zu gleichen Teilen Grundfertigkeiten und sportspezifische Fertigkeiten vermittelt, was die vielfach aufgetauchten Defizite bei der Vermittlung deutlich werden lässt.

7. Rahmenbedingungen und Hilfsmittel liegen in nur sehr unbefriedigendem Ausmaß vor. Hier ist die Administration gefordert - entweder mit einer Adaption des Lehrplans oder mit einer konkreten Lösung der dringendsten Probleme: 1. Anschaffung von Hilfsmitteln für Turnen und Gymnastik, weil diese auch für einen inhaltlich und methodisch variantenreichen Unterricht - insbesondere bei der Fertigkeitsvermittlung - hilfreich wären, 2. Optimierung der Notfallversorgung (möglicherweise durch die Bezirksleiter) sowie Bauen von Sanitäranlagen zur Förderung der Gesundheit, 3. Herstellen der Bedingungen für die Bewertung und Pflege der Sportstätten und möglicherweise 4. Schaffen von Umkleideräumen.

Aus der Strukturanalyse und aus den empirischen Befunden ist zu vermuten, dass die meisten Schüler ein nur sehr niedriges Niveau in den verschiedenen Sportarten aufweisen, was seine Ursachen in der mangelhaften Methodik (kaum gezielte Maßnahmen und nur unzureichende Umsetzung selbst grundlegende methodische Vorgehensweisen) und der fehlenden Sportnote im Zeugnis hat. Eine gezielte Orientierung auf systematische Leistungsverbesserungen (individuell und in Bezug auf das System) findet bei schlecht ausgestatteten Sportstätten kaum statt. Erfolgversprechend für die Ausbildung erscheint eine stärkere Verbindung zwischen Methode und Situation (bzw. Theorie und Praxis), um dies auch später leichter anwenden zu können. Weiterhin kann es fruchtbar sein, zu thematisieren, wie einzelne Methoden in unterschiedlichen bewegungsbezogenen Problemfeldern und bei unterschiedlichen Leistungsniveaus angewandt werden können. Hier sollte die Vermittlung variantenreicher Anwendungen der Ganz-Teil-Methode mit ihren unterschiedlichen Kombinationsmöglichkeiten - und parallel dazu: der effek- 
tive Umgang mit großen Klassen - betont werden. Möglicherweise ist es zur Umsetzung der Erziehungsziele auch hilfreich, in der Sportlehrerausbildung die Präsentation theoretischer Inhalte zu betonen. Dies wäre eine gute Voraussetzung dafür, dass auch bei schlechtem Wetter oder während des Ramadans ein gezielter Theorieunterricht stattfinden kann. Fruchtbare Themen im Hinblick auf die Erziehungsziele und die in der Praxis aufgedeckten Methodendefizite könnten z.B. sein: Gesundheit/Trainingslehre, Taktiken, Informationen über nationale und internationale Meisterschaften und Umgang mit Konflikten.

Insgesamt ist zu folgern, dass die ägyptische Sportpädagogik einen geringen Wissensbestand aufweist. Daher sind perspektivisch ein stärkerer akademischer Austausch (z.B. mit Deutschland), Unterrichtsbeobachtungen, Untersuchungen der Schüler, Übersetzung, Studium und Adaption von deutschsprachigen Konzeptionen (zu Methodik [des Spiels], sozialem Lernen, sportartübergreifender Vermittlung von Sportspielen und Gesundheitserziehung) zu empfehlen. Besonders wichtig ist aber die Einführung einer Sportnote und die Diskussion um weitere Orientierungen. Ein möglicher Weg besteht darin, den Sportunterricht weniger spezifisch und anhand von mehr Sportarten zu unterrichten, sich mehr dem Spiel und allgemeinen grundlegenden Fertigkeiten zu widmen, dafür den Schulsport aber umgekehrt zu einem systematischen Leistungssport auszubauen. 


\section{LITERATURVERZEICHNIS}

AJZEN, I. (1991): The Theory of Planned Behavior. Organizational Behavior and Human Decision Processes, 50, 179-211.

AJZEN, I. (2001): Nature and operation of attitudes. Annual Review of Psychology, 52, 27-58.

ARTUS, H. G. (1974): Jugend und Freizeitsport. Gießen: Achenbach.

BALZ, E. (1993): Wie kann man soziales Lernen fördern? In: BIELEFELDER SPORTPÄDAGOGEN (Hrsg.): Methoden im Sportunterricht. Ein Lehrbuch in 13 Lektionen (S. 131-151, 2. Aufl.). Schorndorf: Hofmann.

BALZ, E. (1997): Wie pädagogisch soll der Schulsport sein? In: E. BALZ \& P. NEUMANN (Hrsg.): Wie pädagogisch soll der Schulsport sein? (S. 7-14). Schorndorf: Hoffmann.

BALZ, E. (2000): Fachdidaktische Entwicklungen. In: H. HAAG \& A. HUMMEL (Hrsg.): Handbuch Sportpädagogik (S. 149-156). Schorndorf: Hofmann.

BECKER, G.E. (1998): Durchführung von Unterricht. Handlungsorientierte Didaktik, Teil II (8. Aufl.). Weinheim: Beltz.

BECKERS, E. (1987): Durch Rückkehr zur Zukunft? Anmerkungen zur Entwicklung der Sportpädagogik. Sportwissenschaft ,17, 241-257.

BORTZ, J. (1999): Statistik für Sozialwissenschaftler (5. Aufl.). Berlin: Springer.

BRÄUTIGAM, M. (2003): Sportdidaktik: Ein Lehrbuch in 12 Lektionen. Aachen: Meyer und Meyer.

BUND, A. (2001): Selbstvertrauen und Bewegungslernen. Studien zur Bedeutung selbstbezogener Kognitionen für das Erlernen (sport)motorischer Fertigkeiten. Schorndorf: Hofmann.

CACHAY, K. (1980): Gruppen und soziale Konflikte im Sport. In: O. GRUPE (Hrsg.): Sport: Theorie in der gymnasialen Oberschule, Band 1 (S. 231-301). Schorndorf: Hofmann. 
CIA (Ed.) (2004): CIA World fact book 2004. Washington, DC: Central Intelligence Agency.

DAHLHAUS, J. (2004): Motivation und Motivierung zum Alterssport. Eingereichte Dissertation an der Sozialwissenschaftlichen Fakultät der Georg-AugustUniversität Göttingen.

DIECKERT, J. \& WOPP, C. (2001): Freizeiterziehung im und durch Sport. In: H. HAAG \& A. HUMMEL (Hrsg.): Handbuch Sportpädagogik (S. 287-293). Schorndorf: Hofmann.

EHNI, H. (2000): Vom Sinn des Schulsports. In: P. WOLTERS, H. EHNI, J. KRETSCHMER, K.-H. SCHERLER, \& W. WEICHERT (Hrsg.): Didaktik des Schulsports (S. 9-35). Schorndorf: Hofmann.

FAVRE, M. (1997): Drei didaktische Ebenen der Leibeserziehung: Mehrdeutigkeit und Unschärfe des ,Begriffs' Didaktik. Sporterziehung in der Schule, 8, 8-11.

FREY, G. (1981): Training im Schulsport. Schorndorf: Hofmann.

GRÖßING, S. (1997): Einführung in die Sportdidaktik: Lehren und Lernen im Sportunterricht. Wiesbaden: Limpert.

HEINEMANN, K. (1980): Einführung in die Soziologie des Sports. Schorndorf: Hofmann

HOTZ, A. (1995): Bewegungen kennen und können - Zur Didaktik einer „bewegungverstehenden" Sportlehrer(innen)ausbildung. In: R. PROHL \& J. SEEWALD (Hrsg.): Bewegung verstehen. Facetten und Perspektiven einer qualitativen Bewegungslehre (S. 181-195). Schorndorf: Hofmann.

KLAFKI, W. (1963): Das pädagogische Problem des Elementaren und die Theorie der kategorialen Bildung. Weinheim: Beltz.

KLOSTERMEIER, I. (1992/93): Polyglott Ägypten. München: Verlag Dr. Bolte KG. KNEBEL, K.-P. (19985): Funktionsgymnastik. Training, Technik, Taktik. Reinbek: rororo. 
KOTTMANN, L. \& KÜPPER, D. (1999): Gesundheitserziehung. In: W. GÜNZEL \& R. LAGING (Hrsg.): Neues Taschenbuch des Sportunterrichts. Bd. 1: Grundlagen und pädagogische Orientierungen (S. 235-252). Baltmannsweiler: Schneider.

KURZ, D. (1998): Lektion 1: Worum geht es in einer Methodik des Sportunterrichts? In: BIELEFELDER SPORTPÄDAGOGEN (Hrsg.): Methoden im Sportunterricht. Ein Lehrbuch in 14 Lektionen (S. 9-24). Schorndorf: Hofmann.

KURZ, D. (1999): Mehrperspektivischer Sportunterricht. In U. PÜHSE \& U. ILLI (Hrsg.): Bewegung und Sport im Lebensraum Schule (S. 90-100). Schorndorf: Hofmann.

LEBERT, H. (1982): Abhängigkeit und Funktion des Sports in Ghana. Diss. An der Ruhr-Universität Bochum.

MEUSEL, H. (1993): Kurze Didaktik der kleinen Bewegungsspiele. Sportpraxis, 34, 49-50.

MEYER, H. (1989): Unterrichtsmethoden, Bd. 2 (2. Aufl.). Berlin: Cornelsen.

PEUKE, R. (1997): Dilemmata einer Methodik sozialen Lernens im Sport und ein möglicher Ansatz. In: G. FRIEDRICH \& E: HILDENBRANDT (Hrsg.): Sportlehrer/in heute - Ausbildung und Beruf. Tagung der dvs-Sektion Sportpaedagogik vom 23. 25.05.1996 im Schloss Rauischholzhausen (S. 225-235). Hamburg: Czwalina.

PÜHSE, U. (1990): Soziales Lernen im Sport. Ein Beitrag zur sportpädagogischen Lernzieldiskussion. Klinkhardt, Bad Heilbrunn.

PÜHSE, U. (1994): Soziales Handeln im Sport und Sportunterricht. Hofmann, Schorndorf.

RAUCH, M. (1995): Merian live - Ägypten, München: Gräfe und Unzer.

RÖHRLE, R./ EMRICH, A./ KOPELMANN, P./ LAU, A./ MAHLITZ, D.-C.I NAGEL, V. \& PETZOLD, R. (1997): Was Sie schon immer über's Sportspiel wissen wollten: Schwerpunkt Methodik. In: K. ROTH \& E.-J. HOSSNER (Hrsg.): Sport - Spiel - Forschung. Zwischen Trainerbank und Lehrstuhl. Sportspiel-Symposium des ISSW Heidelberg und der dvs vom 30.09. - 2.10.1996 in Heidelberg (S. 219232). Hamburg: Czwalina. 
RÖTHIG, P., PROHL, R. u.a. (Hrsg.) (2003): Sportwissenschaftliches Lexikon (7. Aufl.). Schorndorf: Hofmann.

SCHOLL-LATOUR, P. (2002): Kampf dem Terror - Kampf dem Islam? Propyläen, München.

SCHULZ, W. (1972): Unterricht - Analyse und Planung. In: P. HEIMANN, G. OTTO \& W. SCHULZ (Hrsg.): Unterricht. Analyse und Planung (S. 13-47, 6. Aufl.). Hannover: Schroedel.

SEMSEK, H.-G. (1995): Ägypten. Köln: DuMont.

SÖLL, W. (1996): Sportunterricht - Sport unterrichten. Ein Handbuch für Sportlehrer. Schorndorf: Hofmann.

TIBI, B. (1985): Der Islam und das Problem der kulturellen Bewältigung sozialen Wandels. Frankfurt: Suhrkamp.

WENZEL, H. (1996): Bildung und Erziehung in der Sekundarstufe 1 des Gymnasiums. In: W. MAROTZKI, M. MEYER \& H. WENZEL (Hrsg.): Erziehungswissenschaft für Gymnasiallehrer (S. 139-155). Weinheim: Dt. Studien Verlag.

WOLL, A. \& BÖS, K. (2001): Gesundheitserziehung. In: H. HAAG \& A. HUMMEL (Hrsg.): Handbuch Sportpädagogik (S. 294-306). Schorndorf: Hofmann.

YALDAI, S. (1986): Islam und Sport. Religiöse Tradition und moderner Einfluss. Düsseldorfer sportwissenschaftliche Studien, Heft 2, S. 84.

ZARGHAMI, M. (1997): Anthropologische Grundlagen von Spiel und Sport aus der Sicht des Islams. Inaugural-Dissertation der philosophischen Fakultät der Heinrich-Heine-Universität, Düsseldorf. 


\section{Arabischsprachige Literatur}

ABD EL AZIEM, J. (1993): Lehrplan und Methodik. Vorlesungsskript des Sportinstitutes der Zagazig-Universität.

ABD EL AZIZ, S. (1998): Erziehung und Unterrichtsmethoden, Band 1 (5. Aufl.). Kairo: Dar al Ma'arif.

ABD EL HAMID, E. (1998): Studien der Praxis in der Sportwissenschaft. Kairo: Alam al Kutub.

ABD EL HAMID, K. \& EL CHOULI, A. (2000): Enzyklopädie der Olympischen Kul-tur. Kairo: Dar al Ma'arif.

ABD EL HAMID, S. (1999): Verwaltung der Sportorganisationen - moderne Theorie und ihre Anwendungen. Kairo: Dar al Ma'arif.

ABD EL KARIM, A. (1997): Lehrmethoden in der Sportwissenschaft. Alexandria: Munscha'it al Ma'arif.

ABD EL KARIM, A. (1999): Methodik des Sportunterrichts. Alexandria: Munscha'it al Ma'arif.

ABD EL MAQSUD, I. (1996) : Sport und Tourismus. Kairo: Dar al Fikr al Arabi.

ABD EL MAWGUUD, M. (1995): Inhalt und Ordnung der Lehrpläne für den Sportunterricht. Kairo: Alam al Kutub.

ABD EL RAHMAN, E. (1999): Trainingslager aus Sicht der Sportwissenschaft. Kairo: Dar al Ma'arif.

ABU HARGAH, M. \& ZAGHLOUL, S. (1999): Die Lehrpläne des Sportunterrichts. Kairo: Zentrum der Schriftsteller für Veröffentlichung.

ÄGYPTISCHES MINISTERIUM FÜR ERZIEHUNG UND UNTERRICHT (1991):

Das Unterrichtswesen unter Mubarak - ein Ausblick in die Zukunft. Kairo: Matba' Rauz al-Jusuf al gadida.

ÄGYPTISCHES MINISTERIUM FÜR ERZIEHUNG UND UNTERRICHT (1995): Sportlehrplan für Ägypten. Kairo: Matba' Rauz al Jusuf al gadida. 
ÄGYPTISCHES MINISTERIUM FÜR ERZIEHUNG UND UNTERRICHT (1996):

Das nationale Projekt von Mubarak und Erfolge des Unterrichts in den Jahren 1991 bis 1996. Kairo: Matba' Rauz al-Jusuf al gadida.

ÄGYPTISCHES MINISTERIUM FÜR ERZIEHUNG UND UNTERRICHT (1998): Tatsachen der Erziehung: Ziele, Planung, Inhalte und Bewertung. Kairo: Matba' Rauz al-Jusuf al gadida. (Lehrplan)

ÄGYPTISCHES MINISTERIUM FÜR ERZIEHUNG UND UNTERRICHT (2001): Leitlinien für den Sportlehrer / Sport in der Schule. Kairo: Matba' Rauz al-Jusuf al gadida. (Lehrplan)

ÄGYPTISCHES MINISTERIUM FÜR JUGEND UND SPORT (1997): Entwicklung des Sports in Ägypten. Kairo: Matba' Amerja.

ÄGYPTISCHES MINISTERIUM FÜR JUGEND UND SPORT (2000): Entwicklung des Ministeriums von 1952-1998. Kairo: Matba' Amerja.

ÄGYPTISCHES MINISTERIUM FÜR JUGEND UND SPORT (2001): Sportorganisation und Management. Olympische und nicht Olympische Verbände und Sportvereine. Kairo: Matba' Amerja.

ÄGYPTISCHES OLYMPISCHES KOMITEE (2004): History of the mediteranean games. The start of the games. Zugriff am 13.5. 2004 unter http://www.egolympic.org.eg/olymusfram.html

AHMAD, M. (1999): Lehrplan und Lehrmethoden in der Sportwissenschaft. Arabisch. Kairo: Dar al Fikr al Arabi.

AHMAD, B. (1996): Forschung in der Sportwissenschaft. Kairo: Dar alMa'arif.

ALLAWY, H. (1993): Die Lehre des Sporttrainings. Kairo: Dar al Ta'lim (Haus des Bildungswesens).

ALLAWY, H. (1994): Sportspsychologie. Kairo: Dar al Ma'arif.

ALSHARQ ALAWSAT (2004): 300 Millionen Dollar Auslandsdarlehen von den USA an Ägypten. Zeitungsausgabe vom 4.6.2004, Wirtschaftsteil. 
AWIS, M. (1994): Sportbetreuung von ägyptischen Kindern. Kairo: Zentrum für Jugend und Sport.

AZMY, S. (1996): Prinzipien der Sporterziehung zwischen Theorie und Praxis und ihre Bedeutung für das Schulsystem. Alexandria: Universität Alexandria, Dissertation.

BADIR, S. (1998): Lehrpläne für den Sportunterricht. Kairo: Dar al Ma'arif.

BADRAN, S. \& GHAZI, M. (2000): Philosophie der Ausbildung. Kairo: Dar al Ma'arif.

BADRAN, S. (1995): Historische Entwicklung des ägyptischen Bildungssystems. Kairo: Dar al Ma'arif.

BADRAN, S., EL BUHI, F. \& MOHAMMED, L. (2001): Volksschulbildung. Kairo: 'Alam al Kutub.

BAHA'ALDIN, H. (1997): Die zukünftige Wissensvermittlung. Kairo: Dar al Ma'arif.

DARWISH, K. \& EL HAMAHMI, M. (1997): Moderne Ansichten in Erholung und Freizeit. Kairo: Dar al Ma'arif.

DARWISH, K., EL HAMAHMI, M. \& EL MUHANDES, S. (1990): Die Sportverwaltung. Basis und Anwendungen. Kairo: Dar al Ma'arif.

DER HOHE RAT FÜR JUGEND UND SPORT (1997): Studien und Dokumente zur Entwicklung und Betreuung der Jugend und des Sports in Ägypten in der Zeit von 1952 bis 1996. Band 1, Kairo.

DER KORAN (o.J.): El Medina: Druckerei der zwei heiligen Moscheen König Fahads ibn ABD EL Aziz al Saud.

EL ASKALANI, A. (1996): Fath al Bari bi Scharch Sahih al Buchari (Hadith des Buchari in 19 Bänden, Band 12). Kairo: Dar Abi Hajjan.

EL GAMAL, A. (1999): Zusammenhang der Finanzierung bei einigen Sportverbänden in der Arabischen Republik Ägypten. Kairo: Universität Helwan, Institut für Sportwissenschaft, Forschungsbericht. 
EL GAUZIJA, IBN EL QAYIM. (O.J): Die Reiterei. Beirut: Dar al Kutub.

EL GOHARIE, H. (2002): Richtlinien für die Sportlehrerausbildung. EL Mansoura, EL Mansoura-Universität: Habilitationsschrift.

EL HAMAHMI, M. \& ABD EL AZIZ, A. (1998): Erholung zwischen Theorie und Praxis. Kairo: Markaz al Kutub (Buchzentrum).

EL HAMAHMI, M. \& EL CHOULI, A. (1990): Die Basis der Lehrpläne für Sporterziehung. Kairo: Dar al Fikr al Arabi (Haus des arabischen Denkens).

EL LAQANI, A. (1995): Lehrplanentwicklung. Kairo: Alam al Kutub.

EL SHAHED, S. (1997): Lehrmethoden in der Sportwissenschaft. Kairo: El Talaba.

EL CHOULI, A. (1995): Sport und islamische Zivilisation. Geschichtliche und philosophische Studien der islamischen Sportorganisationen. Kairo: Dar al Fikr al Arabi.

EL CHOULI, A. (1996a): Basiswissen für körperliche und sportliche Erziehung. Kairo: Dar al Ma'arif.

EL CHOULI, A . (1996b): Sport und Gesellschaft. Kuwait: 'Alam al Ma'arif.

EL CHOULI, A. (1998): Ursprünge der Leibeserziehung und des Sports: Einführung, Geschichte, Philosophie. Kairo: Dar al Fikr al Arabi.

EL CHOULI, A./ ABD EL FATTAH, M. \& DARWISH, A. (1994): Die schulische Sporterziehung. Kairo: Dar al Fikr al Arabi (Haus des arabischen Denkens).

FARAG, I. (1999): Unterrichtsmethoden im Sportunterricht und Durchführung der Außenaktivitäten. Kairo: Dar al Fikr al arabi.

HASSAN, M. (1996) : Geschichte der Erziehung in Ägypten. Kairo: Buchzentrum. HUMMUS, M. (1997): Leitfaden "Sportwissenschaft lernen“. Alexandria und Kairo: Munscha'it al Ma'aref.

HUSSEIN, A. (1973): Religion und Sport. Alexandria: Universität Alexandria, Dissertation. 
KAMEL, I., SHALTUT, I. \& CHAFAGA, A. (2002): Lehrmethoden in der Sportwissenschaft. Kairo: Al Esha'a.

KARIM, A./ BADRAN, S. \& MOHAMMED, L. (2000): Geschichte der Erziehung und Ausbildung. Kairo: Markaz al Schabab wa al Rijada (Zentrum für Jugend und Sport).

LUTFI, A. (1996): Lehrmethoden in der Sportwissenschaft. Kairo: Al Kutub al Gami'ija.

MADHKUR, A. (1997): Betrachtungen der Erziehungsmethoden. Kairo: Dar al Fikr al Arabi.

MUSTAFA, F. (1998): Ägypten und die Olympische Bewegung (2. Aufl.). Kairo: Dar al Kutub al Misri (Ägyptische Verlagsanstalt).

NAGLA, M. (1993): Experimente innerhalb der Vorbereitungsphase in einer Knabenschule in Alexandria zur Verbesserung des Sportunterrichts. Alexandria: Universität Alexandria, Dissertation.

QILADA, S. (1998 ): Pädagogische Ziele und Lehrplankonzeption. Kairo: Markaz al Kutub (Buchzentrum).

RAYAAN, H. (1992): Erziehung, Philosophie und Bildungspolitik in Ägypten. Kairo: Markaz al Kutub (Buchzentrum).

RIDWAN, N. (1997): Messungen in Sportunterricht und Sportpsychologie. Kairo: Dar al Fikr.

RUSTOM, A. (1994): Die Erziehungsaktivitäten in der Mittelschule nach den Vorschriften Mubaraks. Kairo: Nationales Zentrum für Erziehungsforschung und Entwicklung, Ministerium für Erziehung und Unterricht).

SALIH, S. \& SHEHATA, H. (1994): Der ägyptische Lehrplan - Eine futuristische Sicht . Kairo: Dar al Ma'arif.

SCHANAWI, A. (1987): Die Azhar - Mosche und Universität. Kairo: Maktabat al Anglu. 
SPORTINSTITUT DER EL MANSOURA UNIVERSITÄT (1998): Einführung in die Körper- und Sporterziehung. Vorlesungsskript.

STATISTISCHES ZENTRUM DES MINISTERIUMS FÜR ERZIEHUNG UND UNTERRICHT (2001/2002): Jahrbuch. Kairo.

STATISTISCHES ZENTRUM DES MINISTERIUMS FÜR JUGEND UND SPORT (2001/2002): Jahrbuch. Kairo.

YA'QUUT, A. (1992): Wirkung eines Programms zur allgemeinen Bewegungsförderung und zur Steigerung des Leistungsniveaus für Schüler zwischen 9 und 12 Jahren. Alexandria: Universität Alexandria, Dissertation.

ZAHRAN, D. (1996): Bewertung der Schulleitung im Grundlagenunterricht. Kairo: (Nationales Zentrum für die Bewertung von Schulen) Markaz qaumi li al taqjim al madaris.

ZENTRALES AMT FÜR ÖFFENTLICHE ERHEBUNGEN UND STATISTIK (2001): Statistik der Sportaktivitäten in den Sportorganisationen und des Bildungssektors für das Jahr 1998. Kairo. 


\section{ABBILDUNGSVERZEICHNIS}

Abb. 1 Teilnahme von Schülern und Studenten der Azhar an verschiedenen Sportangeboten (Quelle: ZENTRALES AMT FÜR ÖFFENTLICHE ERHEBUNGEN UND STATISTIK 2001).

Abb. 2 Überblick zum Ägyptischen Bildungssystem.

Abb. 3 Lehreradministration in Ägypten.

Abb. 4 Auszug aus dem Lehrplan der Ägyptischen Regierung (Gymnastische Übungen).

Abb. 5 Auszug aus dem Lehrplan der Ägyptischen Regierung (Stundenbild).

Abb. 6 Altersverteilung der untersuchten Sportlehrkräfte. 


\section{TABELLENVERZEICHNIS}

Tab. 1 Gründungsjahre ägyptischer Sportverbände, die dem Olympischen Komitee angeschlossen sind (nach: ABD EL HAMEED \& EL CHOULI 2000).

Tab. 2 Sportverbände, Sportvereine und Mitglieder in Ägypten (Quelle: ÄGYPTISCHES MINISTERIUM FÜR JUGEND UND SPORT, 2001).

Tab. 3 Anzahl der geförderten Athleten/Mannschaften in verschiedenen Stützpunkten, geordnet nach Sportarten (Quelle: STATISTISCHES ZENTRUM DES MINISTERIUMS FÜR JUGEND UND SPORT, 2001/2002).

Tab. 4 Überblick zur aktuellen Schulsituation (Quelle der Daten, oberer Teil: STATISTISCHES ZENTRUM DES ERZIEHUNGSMINISTERIUMS, 2001/2002).

Tab. 5 Anzahl der Sportlehrerinnen und Sportlehrer in Ägypten (Quelle: STATISTISCHES ZENTRUM DES ERZIEHUNGSMINISTERIUMS, 2001/2002)

Tab. 6 Lehrinhalte des Sportunterrichts in den ersten drei Klassen der Grundschule (Quelle: ÄGYPTISCHES MINISTERIUM FÜR ERZIEHUNG UND UNTERRICHT, 2001)

Tab. 7 Unterrichtsinhalte der 5. Klasse in den sechswöchigen Unterrichtsblöcken (Quelle: ÄGYPTISCHES MINISTE-RIUM FÜR ERZIEHUNG UND UNTERRICHT, 2001)

Tab. 8 Aufbau des Fragebogens

Tab. 9 Zahl der Schulen und Sportlehrkräften im Bezirk El Dakahlia, (Quelle: ZENTRALES AMT FÜR ÖFFENTLICHE ERHEB-UNG UND STATISTIK, 2002) 
Tab. 10 Meinungen der Sportlehrkräfte der Mittelschule zur vorbereitenden Funktion des Sportunterrichts für den Freizeitsport.

Tab. 11 Meinungen der Sportlehrkräfte der Oberschule zur vorbereitenden Funktion des Sportunterrichts für den Freizeitsport.

Tab. 12 Meinungen der Sportlehrkräfte der Mittelschule zur Vermittlung gesundheitlicher Kenntnisse durch den Sportunterricht.

Tab. 13 Meinungen der Sportlehrkräfte der Oberschule zur Vermittlung gesundheitlicher Kenntnisse durch den Sportunterricht.

Tab. 14 Meinungen der Sportlehrkräfte der Mittelschule zum Erziehungsziel Sozialverhalten.

Tab. 15 Meinungen der Sportlehrkräfte der Oberschule zum Erziehungsziel Sozialverhalten.

Tab. 16 Meinungen der Sportlehrkräfte der Mittelschule zur Förderung psychischer Eigenschaften.

Tab. 17 Meinungen der Sportlehrkräfte der Oberschule zur Förderung psychischer Eigenschaften.

Tab. 18 Meinungen der Sportlehrkräfte der Mittelschule zur Vermittlung sportspezifischer Kenntnisse.

Tab. 19 Meinungen der Sportlehrkräfte der Oberschule zur Vermittlung sportspezifischer Kenntnisse.

Tab. 20 Meinungen der Sportlehrkräfte der Mittelschule zur Vermittlung von Freude/ Spaß/ Begeisterung.

Tab. 21 Meinungen der Sportlehrkräfte der Oberschule zur Vermittlung von Freude/ Spaß/ Begeisterung.

Tab. 22 Meinungen der Sportlehrkräfte der Mittelschule zur allgemeinen Methodik. 
Tab. 23 Meinungen der Sportlehrkräfte der Oberschule zur allgemeinen Methodik.

Tab. 24 Meinungen der Sportlehrkräfte der Mittelschule zur Methodik bei der sportartspezifischen Fertigkeitsvermittlung.

Tab. 25 Meinungen der Sportlehrkräfte der Oberschule zur Methodik bei der sportartspezifischen Fertigkeitsvermittlung.

Tab. 26 Meinungen der Sportlehrkräfte der Mittelschule zum individuellen vs. kollektiven Unterrichten.

Tab. 27 Meinungen der Sportlehrkräfte der Oberschule zum individuellen vs. kollektiven Unterrichten.

Tab. 28 Meinungen der Sportlehrkräfte der Mittelschule zu inhaltlichen Schwerpunkten des Sportunterrichts.

Tab. 29 Meinungen der Sportlehrkräfte der Oberschule zu inhaltlichen Schwerpunkten des Sportunterrichts.

Tab. 30 Meinungen der Sportlehrkräfte der Mittelschule zu allgemeinen Bewertungsmethoden.

Tab. 31 Meinungen der Sportlehrkräfte der Oberschule zu allgemeinen Bewertungsmethoden.

Tab. 32 Meinungen der Sportlehrkräfte der Mittelschule zu Merkmalen einer Bewertung.

Tab. 33 Meinungen der Sportlehrkräfte der Oberschule zu Merkmalen einer Bewertung.

Tab. 34 Meinungen der Sportlehrkräfte der Mittelschule zu unbedingt benötigten Rahmenbedingungen.

Tab. 35 Meinungen der Sportlehrkräfte der Oberschule zu unbedingt benötigten Rahmenbedingungen.

Tab. 36 Erlernen selbstständiger Ausführung der Sportarten. 
Tab. 37 Hinweise auf die Möglichkeit zum Freizeitsport (nach Sportarten).

Tab. 38 Erwerb gesundheitlicher Kenntnisse (Zusammenhang zwischen Bewegung und Gesundheit).

Tab. 39 Vermittlung gesundheitlicher Kenntnisse nach Sportarten (Zusammenhang zwischen Bewegung und Gesundheit).

Tab. 40 Erwerb gesundheitlicher Kenntnisse (Zusammenhang zwischen Ernährung und Gesundheit).

Tab. 41 Vermittlung von Kenntnissen über das Herz-Kreislauf-System. 123

Tab. 42 Vermittlung von Kenntnissen über Hygiene.

Tab. 43 Erlernen selbständiger Konfliktlösung (Mittelschule).

Tab. 44 Erlernen selbständiger Konfliktlösung (Oberschule).

Tab. 45 Vermittlung von Zusammenarbeit.

Tab. 46 Vermittlung von Zusammenarbeit (nach Sportarten).

Tab. 47 Akzeptanz von Erfolgen anderer Schüler.

Tab. 48 Förderung des Selbstvertrauens.

Tab. 49 Erwerb von „Kampfgeist“.

Tab. 50 Erwerb weiterer psychischer Eigenschaften (Mittelschule).

Tab. 51 Erwerb weiterer psychischer Eigenschaften (Oberschule).

Tab. 52 Erwerb sportspezifischer Kenntnisse (Mittelschule).

Tab. 53 Erwerb sportspezifischer Kenntnisse (Oberschule).

Tab. 54 Vermittlung vielfältiger Lernerfahrungen.

Tab. 55 Positive Rückmeldungen durch die Sportlehrkräfte.

Tab. 56 Angebot von Hilfe. 
Tab. 57 Angebot von Hilfe (nach Sportarten).

Tab. 58 Sportlehrerinnen und Sportlehrer als „Vorbild durch Begeisterung".

Tab. 59 Vorbild-Sein (nach Sportarten).

Tab. 60 Belohnen besonders guter Leistungen.

Tab. 61 Berücksichtigung von Bedürfnissen der Schüler.

Tab. 62 Vormachen von Übungen.

Tab. 63 Vormachen von Übungen (nach Sportarten).

Tab. 64 Rückmeldung durch die Sportlehrkräfte in ihrem Unterricht.

Tab. 65 Rückmeldung durch die Sportlehrkräfte (nach Sportarten).

Tab. 66 Prüfungsvorbereitungen seitens der Sportlehrkräfte.

Tab. 67 Prüfungsvorbereitungen (nach Sportarten).

Tab. 68 Durchführung von Wettkämpfen (nach Sportarten).

Tab. 69 Verwendete Lehrmethode bei der Fertigkeitsvermittlung.

Tab. 70 Individuelle vs. kollektive Unterrichtsmethoden.

Tab. 71 Inhaltliche Schwerpunkte (Fähigkeiten).

Tab. 72 Inhaltliche Schwerpunkte (Fertigkeiten).

Tab. 73 Bewertungen im Unterricht.

Tab. 74 Bewertungsstile.

Tab. 75 Rahmenbedingungen des ägyptischen Sportunterrichts.

Tab. 76 Bereitstehende Hilfsmittel. 
- $186-$

\section{Anhang}

- Fragebogen

- Lebenslauf

- Danksagung

- Versicherung 


\section{Fragebogen}

Bitte tragen Sie nun dieses Zeichen $(\checkmark)$ bei jeder Frage ein. Bei den meisten Fragen sollten Sie nur ein Zeichen eintragen, bei manchen können Sie auch mehrere eintragen (dies wird dann extra genannt). Tauchen im Fragebogen Punkte auf (.....), antworten Sie bitte kurz in eigenen Worten. Achten Sie bitte darauf, dass Sie jede Frage beantworten - ansonsten wird der Fragebogen bei der Auswertung nicht berücksichtigt werden können.

\section{Persönliche Merkmale}

\section{- Geschlecht und Schulform}

Sie sind....

...Sportlehrer in der Mittelschule

...Sportlehrerin in der Mittelschule

...Sportlehrer in der Oberschule

...Sportlehrerin in der Oberschule

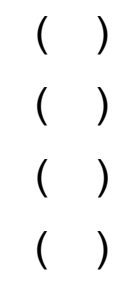

- Alter

Sie sind.... ...zwischen 22 und 30 Jahre alt ...zwischen 31 und 40 Jahre alt ...zwischen 41 und 60 Jahre alt 


\section{(A) Meinungen der Sportlehrerinnen und Sportlehrer}

Im Folgenden wird nach Ihrer persönlichen Meinung gefragt. Es sollen also solche Aussagen getroffen werden, die nur Ihrer ganz eigenen persönlichen Überzeugung entstammen und nicht, was durch andere Personen oder Institutionen als richtig betrachtet wird. Bitte suchen Sie die Aussagen nur nach Ihren ersten Eindruck aus. Bitte tragen Sie nun auch die vorseitig beschriebenen Markierungen ein und beantworten Sie bitte jede Frage.

\section{I: Erziehungsziele}

\section{Freizeit}

1 - Der Sportunterricht ist ein wichtiges Mittel, um die motorischen Voraussetzungen für den Freizeitsport zu legen.

\begin{tabular}{|l|l|l|l|l|}
\hline Stimme völlig zu & Stimme zu & unentschieden & Stimme nicht zu & Stimme überhaupt nicht zu
\end{tabular}

2 - Die Schüler sollen viele verschiedene Sportarten ausüben können.

\begin{tabular}{|l|l|l|l|l|}
\hline Stimme völlig zu & Stimme zu & unentschieden & Stimme nicht zu & Stimme überhaupt nicht zu \\
\hline
\end{tabular}

3 - Die Schüler sollen ihren Freizeitsport selbst organisieren können.

\begin{tabular}{|l|l|l|l|l|}
\hline Stimme völlig zu & Stimme zu & unentschieden & Stimme nicht zu & Stimme überhaupt nicht zu
\end{tabular}

\section{Gesundheit}

4 - Die Schüler sollen den Zusammenhang von Bewegung und Gesundheit kennen. \begin{tabular}{|l|l|l|l|l|}
\hline Stimme völlig zu & Stimme zu & unentschieden & Stimme nicht zu & Stimme überhaupt nicht zu \\
\hline
\end{tabular}

5 - Die Schüler sollen den Zusammenhang von Ernährung und Gesundheit kennen. \begin{tabular}{|l|l|l|l|l|}
\hline Stimme völlig zu & Stimme zu & unentschieden & Stimme nicht zu & Stimme überhaupt nicht zu \\
\hline
\end{tabular}

6 - Die Schüler sollen Kenntnisse über das Herz-Kreislauf-System haben. \begin{tabular}{|l|l|l|l|l|}
\hline Stimme völlig zu & Stimme zu & unentschieden & Stimme nicht zu & Stimme überhaupt nicht zu \\
\hline
\end{tabular} 
7 - Die Schüler sollen Kenntnisse über die gesundheitliche Bedeutung der Hygiene haben.

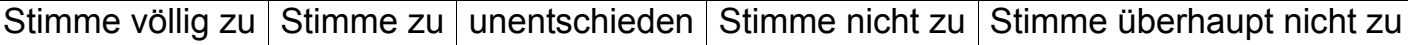

\section{Sozialverhalten}

8 - Der Lehrer muss Konflikte unter den Schülern lösen.

\begin{tabular}{|l|l|l|l|l|}
\hline Stimme völlig zu & Stimme zu & unentschieden & Stimme nicht zu & Stimme überhaupt nicht zu
\end{tabular}

9 - Die Schüler sollen ihre Konflikte selbst lösen.

\begin{tabular}{|l|l|l|l|l|}
\hline Stimme völlig zu & Stimme zu & unentschieden & Stimme nicht zu & Stimme überhaupt nicht zu
\end{tabular}

10 - Die Schüler sollen sprachliche Fähigkeiten zur Konfliktlösung erlernen.

\begin{tabular}{|l|l|l|l|l|}
\hline Stimme völlig zu & Stimme zu & unentschieden & Stimme nicht zu & Stimme überhaupt nicht zu \\
\hline
\end{tabular}

11 - Die Schüler sollen Formen der Zusammenarbeit (z.B. Gruppenarbeit) können. \begin{tabular}{|l|l|l|l|l|}
\hline Stimme völlig zu & Stimme zu & unentschieden & Stimme nicht zu & Stimme überhaupt nicht zu \\
\hline
\end{tabular}

12 - Die Schüler sollen es akzeptieren lernen, wenn sich ein anderer durchsetzt. \begin{tabular}{|l|l|l|l|l|}
\hline Stimme völlig zu & Stimme zu & unentschieden & Stimme nicht zu & Stimme überhaupt nicht zu
\end{tabular}

\section{Psychische Eigenschaften}

13 - Die Schüler sollen Selbstvertrauen bekommen.

\begin{tabular}{|l|l|l|l|l|}
\hline Stimme völlig zu & Stimme zu & unentschieden & Stimme nicht zu & Stimme überhaupt nicht zu \\
\hline
\end{tabular}

14 - Die Schüler sollen „Kampfgeist“ erwerben.

\begin{tabular}{|l|l|l|l|l|}
\hline Stimme völlig zu & Stimme zu & unentschieden & Stimme nicht zu & Stimme überhaupt nicht zu \\
\hline
\end{tabular}

15 - Die Schüler sollen Führungsqualitäten erwerben.

\begin{tabular}{|l|l|l|l|l|}
\hline Stimme völlig zu & Stimme zu & unentschieden & Stimme nicht zu & Stimme überhaupt nicht zu
\end{tabular}

16 - Die Schüler sollen Gehorsam bei Anweisungen und Befehlen lernen.

\begin{tabular}{|l|l|l|l|l|}
\hline Stimme völlig zu & Stimme zu & unentschieden & Stimme nicht zu & Stimme überhaupt nicht zu \\
\hline
\end{tabular} 


\section{Sportspezifische Kenntnisse}

17 - Die Schüler sollen die räumlichen und/oder gerätebezogenen Regeln bei den unterschiedlichen gelehrten Sportarten wissen.

\begin{tabular}{|l|l|l|l|l|}
\hline Stimme völlig zu & Stimme zu & unentschieden & Stimme nicht zu & Stimme überhaupt nicht zu \\
\hline
\end{tabular}

18 - Die Schüler sollen die Spielregeln und taktische Regeln in den unterschiedlichen Sportarten kennen und einhalten.

\begin{tabular}{|l|l|l|l|l|}
\hline Stimme völlig zu & Stimme zu & unentschieden & Stimme nicht zu & Stimme überhaupt nicht zu \\
\hline & & & & \\
\hline
\end{tabular}

19 - Die Schüler sollen über wichtige aktuelle Sportereignisse (z.B. Fußballweltmeisterschaft, Olympische Spiele) informiert werden.

\begin{tabular}{|l|l|l|l|l|}
\hline Stimme völlig zu & Stimme zu & unentschieden & Stimme nicht zu & Stimme überhaupt nicht zu \\
\hline
\end{tabular}

20 - Die Schüler sollen möglichst viele eigene Lernerfahrungen sammeln.

\begin{tabular}{|l|l|l|l|l|}
\hline Stimme völlig zu & Stimme zu & unentschieden & Stimme nicht zu & Stimme überhaupt nicht zu
\end{tabular}

\section{Freude/Spaß/Begeisterung:}

21 - Der Lehrer sollte möglichst viele positive Rückmeldungen geben, um die Schüler Stolz auf ihre sportlichen Leistungen zu machen.

\begin{tabular}{|l|l|l|l|l|}
\hline Stimme völlig zu & Stimme zu & unentschieden & Stimme nicht zu & Stimme überhaupt nicht zu \\
\hline & & & & \\
\hline
\end{tabular}

22 - Der Lehrer sollte immer Hilfe anbieten.

\begin{tabular}{|l|l|l|l|l|}
\hline Stimme völlig zu & Stimme zu & unentschieden & Stimme nicht zu & Stimme überhaupt nicht zu \\
\hline
\end{tabular}

23 - Der Lehrer sollte ein gutes Vorbild sein, indem er seine Begeisterung für den Sport zum Ausdruck bringt.

\begin{tabular}{|l|l|l|l|l|}
\hline Stimme völlig zu & Stimme zu & unentschieden & Stimme nicht zu & Stimme überhaupt nicht zu \\
\hline
\end{tabular}

24 - Die Bedürfnisse der Schüler sind zu berücksichtigen.

\begin{tabular}{|l|l|l|l|l|}
\hline Stimme völlig zu & Stimme zu & unentschieden & Stimme nicht zu & Stimme überhaupt nicht zu
\end{tabular} 


\section{Methodisches Vorgehen}

Im Lehrplan werden für die Vermittlung motorischer Fähigkeiten und Fertigkeiten verschiedene Vorgaben gemacht. Bedenken Sie bitte bei der Beantwortung der folgenden Fragen nochmals, dass lhre Meinung gefragt ist und nicht, ob Sie den Lehrplan in Ihrem Unterricht beachten.

\section{Allgemeine Methodik}

25 - Es ist wichtig, dass der Lehrer in der Praxis immer auch die verschiedenen Übungen vormacht.

\begin{tabular}{|l|l|l|l|l|}
\hline Stimme völlig zu & Stimme zu & unentschieden & Stimme nicht zu & Stimme überhaupt nicht zu \\
\hline & & & & \\
\hline
\end{tabular}

26 - Der Lehrer muss die Schüler anleiten. Das muss in jeder Situation so sein.

\begin{tabular}{|l|l|l|l|l|}
\hline Stimme völlig zu & Stimme zu & unentschieden & Stimme nicht zu & Stimme überhaupt nicht zu \\
\hline & & & & \\
\hline
\end{tabular}

27 - In den überwiegenden Sportspielsituationen sollen die Schüler (u.a. mit Hilfe des Lehrers) lernen, selbständig Problemlösungen zu finden.

\begin{tabular}{|l|l|l|l|l|}
\hline Stimme völlig zu & Stimme zu & unentschieden & Stimme nicht zu & Stimme überhaupt nicht zu \\
\hline
\end{tabular}

28 - Der Lehrer soll immer Rückmeldungen (richtig - falsch) während der Ausbildung geben.

\begin{tabular}{|l|l|l|l|l|}
\hline Stimme völlig zu & Stimme zu & unentschieden & Stimme nicht zu & Stimme überhaupt nicht zu
\end{tabular}

29 - Prüfungen sollten nicht nur für den Lehrer sein, sondern auch eine wichtige Methoden, um den Lernerfolg der Schüler zu sichern.

\begin{tabular}{|l|l|l|l|l|}
\hline Stimme völlig zu & Stimme zu & unentschieden & Stimme nicht zu & Stimme überhaupt nicht zu \\
\hline & & & & \\
\hline
\end{tabular}

30 - Die Schüler sollen sich selbständig (im Rahmen der Ausbildung) auf die Prüfung vorbereiten.

\begin{tabular}{|l|l|l|l|l|}
\hline Stimme völlig zu & Stimme zu & unentschieden & Stimme nicht zu & Stimme überhaupt nicht zu \\
\hline
\end{tabular}

31- Wettkämpfe mit anderen Schulen sind wichtig.

\begin{tabular}{|l|l|l|l|l|}
\hline Stimme völlig zu & Stimme zu & unentschieden & Stimme nicht zu & Stimme überhaupt nicht zu \\
\hline
\end{tabular} 
32 - Die Schüler sollen in allen Sportarten Wettkämpfe durchführen.

\begin{tabular}{|l|l|l|l|l|}
\hline Stimme völlig zu & Stimme zu & unentschieden & Stimme nicht zu & Stimme überhaupt nicht zu
\end{tabular}

33 - Die Schüler sollen in jedem Fall sportgerecht gekleidet sein.

\begin{tabular}{|l|l|l|l|l|}
\hline Stimme völlig zu & Stimme zu & unentschieden & Stimme nicht zu & Stimme überhaupt nicht zu \\
\hline
\end{tabular}

\section{Methodik bei der sportartspezifischen Fertigkeitsvermittlung}

34 - Welche Lehrmethoden passen bei der Vermittlung sportartspezifischer Fertigkeiten zu Ihren Schülern? (Kreuzen Sie nur eine Möglichkeit an)

- Nur die Ganzheitsmethode

- Nur die Teilmethode

- Nur die Ganz-Teil-Methode

- Sowohl die Ganzheitsmethode als auch die Teilmethode

- Sowohl die Ganzheitsmethode als auch Ganz-Teil-Methode

- Sowohl die Teilmethode als auch die Ganz -Teil-Methode

- Alle Methoden

- Andere Methoden

35 - In den überwiegenden Situationen sind individuelle Lehrmethoden wesentlich. \begin{tabular}{|l|l|l|l|l|}
\hline Stimme völlig zu & Stimme zu & unentschieden & Stimme nicht zu & Stimme überhaupt nicht zu \\
\hline
\end{tabular}

36 - In den überwiegenden Situationen sind kollektive Lehrmethoden wesentlich.

\begin{tabular}{|l|l|l|l|l|}
\hline Stimme völlig zu & Stimme zu & unentschieden & Stimme nicht zu & Stimme überhaupt nicht zu \\
\hline
\end{tabular}

\section{Inhaltliche Schwerpunkte}

37 - Die Schüler sollen vor allem Kraft ausbilden.

\begin{tabular}{|l|l|l|l|l|}
\hline Stimme völlig zu & Stimme zu & unentschieden & Stimme nicht zu & Stimme überhaupt nicht zu \\
\hline
\end{tabular} 
38 - Die Schüler sollen vor allem Ausdauer ausbilden.

\begin{tabular}{|l|l|l|l|l|}
\hline Stimme völlig zu & Stimme zu & unentschieden & Stimme nicht zu & Stimme überhaupt nicht zu
\end{tabular}

39 - Die Schüler sollen vor allem Beweglichkeit erlangen.

\begin{tabular}{|l|l|l|l|l|}
\hline Stimme völlig zu & Stimme zu & unentschieden & Stimme nicht zu & Stimme überhaupt nicht zu
\end{tabular}

40 - Die Schüler sollen vor allem Schnelligkeit erlangen.

\begin{tabular}{|l|l|l|l|l|}
\hline Stimme völlig zu & Stimme zu & unentschieden & Stimme nicht zu & Stimme überhaupt nicht zu
\end{tabular}

41 - Die Schüler sollen vor allem koordinative Fähigkeiten ausbilden.

\begin{tabular}{|l|l|l|l|l|}
\hline Stimme völlig zu & Stimme zu & unentschieden & Stimme nicht zu & Stimme überhaupt nicht zu \\
\hline
\end{tabular}

42 - Die Schüler sollen vor allem motorische Grundfertigkeiten erwerben (wie Werfen, Springen, Drehen, Treffen).

\begin{tabular}{|l|l|l|l|l|}
\hline Stimme völlig zu & Stimme zu & unentschieden & Stimme nicht zu & Stimme überhaupt nicht zu \\
\hline
\end{tabular}

43 - Die Schüler sollen vor allem sportartspezifische Fertigkeiten erwerben (z.B. Kopfball im Fußball, Sprungwurf im Handball, Korbleger im Basketball, Fosbury-Flop).

\begin{tabular}{|l|l|l|l|l|}
\hline Stimme völlig zu & Stimme zu & unentschieden & Stimme nicht zu & Stimme überhaupt nicht zu
\end{tabular}

\section{Bewertungsmethoden}

44 - Welche Bewertungsmethoden finden Sie wichtig?

- Regelmäßige Bewertung

\begin{tabular}{|l|l|l|l|l|}
\hline Sehr wichtig & wichtig & unentschieden & unwichtig & Völlig unwichtig \\
\hline
\end{tabular}

- Zwischenbewertung

\begin{tabular}{|l|l|l|l|l|}
\hline Sehr wichtig & wichtig & unentschieden & unwichtig & Völlig unwichtig \\
\hline & & & & \\
\hline
\end{tabular}

- Endbewertung

\begin{tabular}{|l|l|l|l|l|}
\hline Sehr wichtig & wichtig & unentschieden & unwichtig & Völlig unwichtig \\
\hline
\end{tabular} 
45 - Welche Bewertungsinhalte sollten bei Prüfungen berücksichtigt werden? (Mehrfachnennung möglich)

- Beobachtungsmerkmale

- Vorhergehende unterrichtsbegleitende Notizen

- Gesundheitszustand

- Soziale Bedingungen

\section{Rahmenbedingungen und Hilfsmittel}

46 - Welche Rahmebedingungen benötigen Sie unbedingt für Ihren Sportunterricht?

- Regelmäßige Wartung der Sportstätten und der dazugehörigen Einrichtungen wie Gegenstände

\begin{tabular}{|c|l|l|c|c|}
\hline $\begin{array}{c}\text { Benötige ich auf jeden } \\
\text { Fall }\end{array}$ & Benötige ich & unentschieden & $\begin{array}{c}\text { Benötige ich eher } \\
\text { nicht }\end{array}$ & $\begin{array}{c}\text { Benötige ich überhaupt } \\
\text { nicht }\end{array}$ \\
\hline & & & & \\
\hline
\end{tabular}

- Adäquater Umkleideraum

\begin{tabular}{|c|l|l|c|c|}
\hline $\begin{array}{c}\text { Benötige ich auf jeden } \\
\text { Fall }\end{array}$ & Benötige ich & unentschieden & $\begin{array}{c}\text { Benötige ich eher } \\
\text { nicht }\end{array}$ & $\begin{array}{c}\text { Benötige ich überhaupt } \\
\text { nicht }\end{array}$ \\
\hline & & & & \\
\hline
\end{tabular}

- Ausreichende Anzahl von Toiletten und Waschbecken

\begin{tabular}{|c|l|l|c|c|}
\hline $\begin{array}{c}\text { Benötige ich auf jeden } \\
\text { Fall }\end{array}$ & Benötige ich & unentschieden & $\begin{array}{c}\text { Benötige ich eher } \\
\text { nicht }\end{array}$ & $\begin{array}{c}\text { Benötige ich überhaupt } \\
\text { nicht }\end{array}$ \\
\hline & & & & \\
\hline
\end{tabular}

- Ärztliche Betreuung bei Krankheitsfällen / Unfällen der Schüler

\begin{tabular}{|c|c|c|c|c|}
\hline $\begin{array}{c}\text { Benötige ich auf jeden } \\
\text { Fall }\end{array}$ & Benötige ich & unentschieden & $\begin{array}{c}\text { Benötige ich eher } \\
\text { nicht }\end{array}$ & $\begin{array}{c}\text { Benötige ich überhaupt } \\
\text { nicht }\end{array}$ \\
\hline & & & & \\
\hline
\end{tabular}

- Eine Mahlzeit pro Tag für die Schüler

\begin{tabular}{|c|l|l|c|c|}
\hline $\begin{array}{c}\text { Benötige ich auf jeden } \\
\text { Fall }\end{array}$ & Benötige ich & unentschieden & $\begin{array}{c}\text { Benötige ich eher } \\
\text { nicht }\end{array}$ & $\begin{array}{c}\text { Benötige ich überhaupt } \\
\text { nicht }\end{array}$ \\
\hline & & & & \\
\hline
\end{tabular}

- Ein extra Budget für den Sportunterricht

\begin{tabular}{|c|l|l|c|c|}
\hline $\begin{array}{c}\text { Benötige ich auf jeden } \\
\text { Fall }\end{array}$ & Benötige ich & unentschieden & $\begin{array}{c}\text { Benötige ich eher } \\
\text { nicht }\end{array}$ & $\begin{array}{c}\text { Benötige ich überhaupt } \\
\text { nicht }\end{array}$ \\
\hline & & & & \\
\hline
\end{tabular}




\section{(B) Praxis des Sportunterrichts}

Im Folgenden wird nach Ihrer persönlichen alltäglichen Praxis gefragt. Es sollen also solche Angaben gemacht werden, die möglichst genau Ihren Sportunterricht beschreiben. Bitte suchen Sie die Aussagen auch hier nach Ihren ersten Gedanken

aus. Bitte tragen Sie nun dieses Zeichen $(\checkmark)$ bei jeder Frage ein und gegebenenfalls eine kurze Beschreibung bei den Punkten (.....). Bei den meisten Fragen sollten Sie nur ein Zeichen eintragen, bei manchen können Sie auch mehrere eintragen (dies wird dann extra genannt). Achten Sie bitte auch hier wieder darauf, dass Sie jede Frage beantworten - ansonsten wird der Fragebogen bei der Auswertung nicht berücksichtigt werden können.

\section{I: Erziehungsziele}

\section{Freizeit}

47 - Lernen die Schüler, die Sportarten auch in ihrer Freizeit selbständig, d.h. ohne Sie, auszuüben?

$\mathrm{Ja}$

Vielleicht

Nein

- Falls Sie „ja“ oder „vielleicht“ angekreuzt haben: Welche Maßnahmen unternehmen Sie in Ihrem Unterricht dazu?

48 - Bei welchen Sportarten weisen Sie auf Möglichkeiten zur Freizeitausübung hin? (Mehrfachnennung möglich)

- Fußball

- Handball

- Basketball

- Volleyball

- Leichtathletik

- Turnen 


\section{Gesundheit}

49 - Lernen die Schüler etwas über den Zusammenhang von Bewegung und Gesundheit?

Ja

Vielleicht

Nein

- Falls Sie „ja“ oder „vielleicht“ angekreuzt haben: Welche Maßnahmen unternehmen Sie in Ihrem Unterricht dazu?

50 - Bei welchen Sportarten verdeutlichen Sie solche Zusammenhänge?

(Mehrfachnennung möglich)

- Gymnastik

- Fußball

- Handball

- Basketball

- Volleyball

- Leichtathletik

- Turnen

51 - Lernen die Schüler etwas über den Zusammenhang zwischen Ernährung und Gesundheit?

$\mathrm{Ja}$

Vielleicht

Nein

- Falls Sie „ja“ oder „vielleicht“ angekreuzt haben: Welche Maßnahmen unternehmen Sie in Ihrem Unterricht dazu? 
52 - Vermitteln Sie Kenntnisse über das Herz-Kreislauf-System?

$\mathrm{Ja}$

Nein

- Falls Sie „ja“ angekreuzt haben: Welche Maßnahmen unternehmen Sie in Ihrem Unterricht dazu?

53 - Lernen die Schüler die gesundheitliche Bedeutung der Hygiene kennen?

$\mathrm{Ja}$

Vielleicht

Nein

- Falls Sie „ja“ oder „vielleicht“ angekreuzt haben: Welche Maßnahmen unternehmen Sie in Ihrem Unterricht dazu?

\section{Sozialverhalten}

54 - Lernen die Schüler ihre Konflikte selbst zu lösen?

Ja

Nein

- Falls Sie „ja“ angekreuzt haben: Welche Maßnahmen unternehmen Sie in Ihrem Unterricht dazu?

55 - Vermitteln Sie den Schüler sprachliche Fähigkeiten zur Konfliktlösung?

$\mathrm{Ja}$

Nein

- Falls Sie „ja“ angekreuzt haben: Welche Maßnahmen unternehmen Sie in Ihrem Unterricht dazu? 
56 - Lernen die Schüler zusammen zu arbeiten ?

$\mathrm{Ja}$

Vielleicht

Nein

- Falls Sie „ja“ oder „vielleicht“ angekreuzt haben: Welche Maßnahmen unternehmen Sie in Ihrem Unterricht dazu?

57 - Bei welchen Sportarten fördern Sie die Zusammenarbeit?

(Mehrfachnennung möglich)

- Gymnastik

- Fußball

- Handball

- Basketball

- Volleyball

- Leichtathletik

- Turnen

58 - Lernen die Schüler das Durchsetzen eines anderen zu akzeptieren?

$\mathrm{Ja}$

Vielleicht

Nein

- Falls Sie „ja“ oder „vielleicht“ angekreuzt haben: Welche Maßnahmen unternehmen Sie in Ihrem Unterricht dazu?

\section{Psychische Eigenschaften}

59 - Fördern Sie gezielt das Selbstvertrauen Ihrer Schüler?

$\mathrm{Ja}$

Nein

Das Selbstvertrauen wird automatisch gestärkt 
60 - Erwerben die Schüler „Kampfgeist“?

$\mathrm{Ja}$

Nein

- Falls Sie „ja“ angekreuzt haben: Welche Maßnahmen unternehmen Sie in Ihrem Unterricht dazu?

61 - Erwerben die Schüler Führungsqualitäten?

$\mathrm{Ja}$

Vielleicht

Nein

- Falls Sie „ja“ oder "vielleicht“ angekreuzt haben: Welche Maßnahmen unternehmen Sie in Ihrem Unterricht dazu?

62 - Lernen die Schüler Gehorsam bei Anweisungen und Befehlen?

$\mathrm{Ja}$

Nein

- Falls Sie „ja“ angekreuzt haben: Welche Maßnahmen unternehmen Sie in Ihrem Unterricht dazu?

63 - Lernen die Schüler, sich zu bemühen?

Ja

Vielleicht

Nein

- Falls Sie „ja“ oder „vielleicht“ angekreuzt haben: Welche Maßnahmen unternehmen Sie in Ihrem Unterricht dazu? 


\section{Sportspezifische Kenntnisse}

64 - Lernen die Schüler die räumlichen und/oder gerätebezogenen Regeln bei den unterschiedlichen Sportarten?

$\mathrm{Ja}$

Vielleicht

Nein

- Falls Sie „ja“ oder "vielleicht“ angekreuzt haben: Welche Maßnahmen unternehmen Sie in Ihrem Unterricht dazu?

65 - Lernen die Schüler die Einhaltung von Spielregeln und von taktischen Regeln?

$\mathrm{Ja}$

Vielleicht

Nein

- Falls Sie „ja“ oder "vielleicht“ angekreuzt haben: Welche Maßnahmen unternehmen Sie in Ihrem Unterricht dazu?

66 - Berichten Sie den Schüler über wichtige aktuelle Sportereignisse (z.B. Fußballweltmeisterschaft, Olympische Spiele)?

Ja

Nein

67 - Gestalten Sie Ihren Sportunterricht so, dass die Schüler möglichst viele eigene Lernerfahrungen sammeln?

Ja

Nein

- Falls Sie „ja“ angekreuzt haben: Welche Maßnahmen unternehmen Sie in Ihrem Unterricht dazu? 


\section{Freude/Spaß/Begeisterung}

68 - Geben Sie möglichst viele positive Rückmeldungen, um die Schüler stolz auf ihre sportlichen Handlungen zu machen?

Ja

Nein

- Falls Sie „ja“ angekreuzt haben: Welche Maßnahmen unternehmen Sie in Ihrem Unterricht dazu?

69 - Bieten Sie Hilfe an?

Immer

Meistens

Manchmal

Nie

- Falls nicht "nie“ angekreuzt haben: Welche Maßnahmen unternehmen Sie in Ihrem Unterricht dazu?

70 - Bei welchen Sportarten bieten Sie Hilfe an? (Mehrfachnennung möglich)

- Gymnastik

- Fußball

- Handball

- Basketball

- Volleyball

- Leichtathletik

- Turnen 
71 - Sind Sie ein gutes Vorbild, indem Sie Ihre Begeisterung für den Sport zum Ausdruck bringen?

Ja, immer

Meistens

Manchmal

$\mathrm{Nie}$

- Falls nicht "nie“ angekreuzt haben: Welche Maßnahmen unternehmen Sie in Ihrem Unterricht dazu?

72 - Bei welchen Sportarten sind Sie ein gutes Vorbild und begeistern die Schüler dadurch? (Mehrfachnennung möglich)

- Gymnastik

- Fußball

- Handball

- Basketball

- Volleyball

- Leichtathletik

- Turnen

73 - Belohnen Sie besonders gute Leistungen?

$\mathrm{Ja}$

Nein

- Falls Sie „ja“ angekreuzt haben: Welche Maßnahmen unternehmen Sie in Ihrem Unterricht dazu? 
74 - Berücksichtigen Sie die Bedürfnisse der Schüler?

Immer

Meistens

Manchmal

$\mathrm{Nie}$

- Falls nicht "nie“ angekreuzt haben: Welche Maßnahmen unternehmen Sie in Ihrem Unterricht dazu?

\section{Methodisches Vorgehen}

\section{Allgemeine Methodik}

75 - Machen Sie in der Praxis die verschiedenen Übungen vor?

Immer

Meistens

Manchmal

Nie

76 - Bei welcher Sportart machen Sie Übungen vor?

(Mehrfachnennung möglich)

- Gymnastik

- Fußball

- Handball

- Basketball

- Volleyball

- Leichtathletik

- Turnen 
77 - Geben Sie Rückmeldungen (richtig - falsch) während der Übungen?

Immer

Meistens

Manchmal

Nie

- Falls nicht "nie“ angekreuzt haben: Welche Maßnahmen unternehmen Sie in Ihrem Unterricht dazu?

78 - Bei welcher Sportart geben Sie Rückmeldungen?

(Mehrfachnennung möglich)

- Gymnastik

- Fußball

- Handball

- Basketball

- Volleyball

- Leichtathletik

- Turnen

79 - Bereiten Sie die Schüler auf die Prüfungen vor?

Immer

Meistens

Manchmal

Nie

- Falls nicht "nie“ angekreuzt haben: Welche Maßnahmen unternehmen Sie in Ihrem Unterricht dazu? 
80 - Bei welcher Sportart bereiten sie die Schüler auf die Prüfung vor? (Mehrfachnennung möglich)

- Fußball

- Handball

- Basketball

- Volleyball

- Leichtathletik

- Turnen

81 - Bei welcher Sportart führen Sie Wettkämpfe durch?

(Mehrfachnennung möglich)

- Fußball

- Handball

- Basketball

- Volleyball

- Leichtathletik

- Turnen

\section{Methodik bei der sportartspezifischen Fertigkeitsvermittlung}

82 - Welche Lehrmethoden setzen Sie bei der Vermittlung der im Lehrplan vorgesehenen Sportarten ein? (Mehrfachnennung möglich)

- Nur die Ganzheitsmethode

- Nur die Teilmethode

- Nur die Ganz-Teil-Methode

- Sowohl die Ganzheitsmethode als auch die Teilmethode

- Sowohl die Ganzheitsmethode als auch Ganz-Teil-Methode

- Sowohl die Teilmethode als auch die Ganz -Teil-Methode

- Alle Methoden

- Andere Methoden 
83 - Welche Lehrmethoden setzen Sie überwiegenden ein?

- Individuelle

- Kollektive

\section{Inhaltliche Schwerpunkte}

84 - Welche Fähigkeiten fördern Sie besonders ausgeprägt?

(Mehrfachnennung möglich)

- Ausdauerfähigkeiten

- Beweglichkeit

- Schnelligkeit

- Koordinative Fähigkeiten

85 - Gibt es bei der Vermittlung von Fertigkeiten in Ihrem Unterricht Schwerpunkte?

- Ich vermittle mehr motorische Grundfertigkeiten (wie Werfen, Springen, Drehungen, Treffen).

- Ich vermittle mehr sportartspezifische Fertigkeiten

(z.B. Kopfball im Fußball, Sprungwurf im Handball, Korbleger im Basketball, Fosbury-Flop).

- Ich vermittle beide Fertigkeiten zu gleichen Teilen.

\section{Bewertungsmethoden}

86 - Bewerten sie den Leistungsstand oder die Leistungsentwicklung der Schüler nach objektiven Kriterien?

$\mathrm{Ja}$

Nein

- Falls sie „ja“" angekreuzt haben: weiter mit Frage 87.

- Falls sie „nein“ angekreuzt haben: weiter mit Frage 88. 
87 - Wie kommen Sie zum Bewertungsergebnis?

- Durch regelmäßige Zwischenbewertungen

- Durch Zwischenbewertungen und Endbewertung

- Durch Endbewertung

\section{Rahmenbedingungen und Hilfsmittel}

88 - Welche Rahmenbedingungen liegen tatsächlich für Ihren Sportunterricht vor? (Mehrfachnennung möglich)

- Regelmäßige Wartung der Sportstätten und der dazugehörigen Einrichtungen wie Gegenstände

- Adäquater Umkleideraum

- Ausreichende Anzahl von Toiletten und Waschbecken

- Ärztliche Betreuung bei Krankheitsfällen / Unfällen der Schüler

- Mehrere Mahlzeiten pro Woche für die Schüler

- Ein extra Budget für den Sportunterricht 
89 - Welche Hilfsmittel liegen für Ihren Sportunterricht tatsächlich vor? (Mehrfachnennung möglich)

- Turnmatten

- Fußbälle / Handbälle / Basketbälle / Volleybälle incl. Pumpe

- Ersatzbälle

- Medizinbälle

- Hürden

- Springseile

- Gymnastikbänke

- Kasten / Pferd

- Keulen / Reifen / Stäbe

- Fußballtore

- Handballtore

- Basketballkörbe

- Volleyballnetz incl. Pfosten

- Kugeln (Kugelstoß)

- Diskus/Speer

- Sandgrube

- Lange Maßbänder 


\section{Lebenslauf}

Name: $\quad$ Osman

Vorname: $\quad$ Ashraf Osman Abd El Motleb

Geburtsdatum: 27.03.1971

Geburtsort: $\quad$ EL Mansoura / Ägypten

Nationalität: $\quad$ Ägypter

Familienstand: verheiratet und Vater zweier Kinder

\section{Ausbildung}

1985-1988: $\quad$ Oberschule in EL Mansoura / Ägypten

1988-1992: Hauptstudium an der Fakultät für Sportwissenschaften der Zagazig Universität (Al Sharqia / Ägypten). Abschlussnote: ausgezeichnet.

1996-1999: $\quad$ Magisterarbeit an der Fakultät für Sportwissenschaften der Zagazig - Universität Abschlussnote: sehr gut.

seit 2000: $\quad$ Promotion an der Fakultät für Sozialwissenschaften der Georg-August-Universität Göttingen (Stipendiat der Ägyptischen Regierung).

\section{Berufspraxis}

1996-1998: Assistent an der Fakultät für Sportwissenschaften der EL Mansora - Universität (Ägypten)

1999-2000: Oberassistent an der Fakultät für Sportwissenschaften der EL Mansora Universität 


\section{Danksagung}

Mein Dank gilt an erster Stelle Gott, der mir die Geduld und die Fähigkeit geschenkt hat, diese Arbeit zu erstellen. Die Arbeit wäre allerdings ohne die Mitwirkung vieler Beteiligter nie entstanden.

Daher gilt mein Dank vor allem meinem Doktorvater, Prof. Dr. HERBERT HOPF, der für unsere vierjährige Zusammenarbeit die notwendigen Arbeitsbedingungen zur Erstellung dieser Dissertation schaffte und mich mit zahlreichen richtungweisenden und fachkundigen Anregungen unterstützte.

Weiterhin möchte ich Prof. Dr. JÜRGEN SCHRÖDER und Prof. Dr. HANSDIETER HALLER danken, die als Gutachter bzw. Prüfer meinem Vorhaben stets aufgeschlossen gegenüberstanden.

Ein ganz spezielles Dankeschön richte ich an zwei Personen, die durch ihre Beiträge in fruchtbaren wissenschaftlichen Diskussionen nicht nur einen wichtigen Einfluss auf diese Arbeit ausgeübt, sondern mich auch selbst positiv beeinflusst haben: Prof. Dr. MOHAMED KESHK und JÖRG DAHLHAUS.

Bedanken möchte ich mich auch bei den ägyptischen Sportlehrern und Sportlehrerinnen, die an dieser Untersuchung teilnahmen. Ohne ihren Einsatz hätte diese Arbeit nicht entstehen können.

Schließlich gilt mein besonderer Dank meiner Ehefrau NAGLAA für ihre Unterstützung, Geduld und Toleranz während der Fertigstellung dieser Arbeit.

Ashraf Osman

Göttingen, im September 2004 


\section{Versicherung}

Ich versichere, dass ich die eingereichte Dissertation „Analyse des Sportunterrichts in der Arabischen Republik Ägypten" selbständig und ohne unerlaubte Hilfsmittel verfasst habe. Anderer als der von mir angegebenen Hilfsmittel und Schriften habe ich mich nicht bedient. Alle wörtlich oder sinngemäß den Schriften anderer Autoren entnommenen Stellen habe ich kenntlich gemacht.

Göttingen, den 03.09. 2004 